OAK RIDGE

ORNL/TM-2009/305

NATIONAL LABORATORY

MANAGED BY UT-BATTELLE

FOR THE DEPARTMENT OF ENERGY

\title{
Data Compilation for AGR-2 UCO Baseline Compact Lot LEU07-OP1-Z
}

Revision 0

\author{
John D. Hunn, Fred C. Montgomery and \\ Peter J. Pappano
}

December 2009

\author{
Prepared for the \\ United States Department of Energy - \\ Office of Nuclear Energy \\ under the \\ Next Generation Nuclear Plant - \\ Advanced Gas Reactor Fuel Development Program
}




\section{DOCUMENT AVAILABILITY}

Reports produced after January 1, 1996, are generally available free via the U.S. Department of Energy (DOE) Information Bridge.

Web site http://www.osti.gov/bridge

Reports produced before January 1, 1996, may be purchased by members of the public from the following source.

National Technical Information Service

5285 Port Royal Road

Springfield, VA 22161

Telephone 703-605-6000 (1-800-553-6847)

TDD 703-487-4639

Fax 703-605-6900

E-mail info@ntis.fedworld.gov

Web site http://www.ntis.gov/support/ordernowabout.htm

Reports are available to DOE employees, DOE contractors, Energy Technology Data Exchange (ETDE) representatives, and International Nuclear Information System (INIS)

representatives from the following source.

Office of Scientific and Technical Information

P.O. Box 62

Oak Ridge, TN 37831

Telephone 865-576-8401

Fax 865-576-5728

E-mail reports@adonis.osti.gov

Web site http://www.osti.gov/contact.html

This report was prepared as an account of work sponsored by an agency of the United States Government. Neither the United States Government nor any agency thereof, nor any of their employees, makes any warranty, express or implied, or assumes any legal liability or responsibility for the accuracy, completeness, or usefulness of any information, apparatus, product, or process disclosed, or represents that its use would not infringe privately owned rights. Reference herein to any specific commercial product, process, or service by trade name, trademark, manufacturer, or otherwise, does not necessarily constitute or imply its endorsement, recommendation, or favoring by the United States Government or any agency thereof. The views and opinions of authors expressed herein do not necessarily state or reflect those of the United States Government or any agency thereof. 


\title{
Data Compilation for AGR-2 UCO Baseline Compact Lot LEU07-OP1-Z
}

\author{
J. D. Hunn, F. C. Montgomery and P. J. Pappano \\ Oak Ridge National Laboratory
}

This document is a compilation of characterization data for the candidate AGR-2 UCO Baseline fuel compact lot LEU07-OP1-Z. The compacts were produced by ORNL for the Advanced Gas Reactor Fuel Development and Qualification (AGR) program for the second AGR irradiation test (AGR-2). This compact lot was fabricated using particle composite LEU07. LEU07 came from Babcock and Wilcox (B\&W) coated particle lot G73J-14-93072A, which was an upgraded batch of TRISO-coated $425 \mu \mathrm{m}$ diameter, $14 \%$ low enrichment uranium oxide/uranium carbide kernels (LEUCO). The AGR-2 TRISO-coated particles consist of a spherical kernel coated with an $\sim 50 \%$ dense carbon buffer layer (100 $\mu \mathrm{m}$ nominal thickness), followed by a dense inner pyrocarbon layer (40 $\mu \mathrm{m}$ nominal thickness), followed by a $\mathrm{SiC}$ layer $(35 \mu \mathrm{m}$ nominal thickness), followed by another dense outer pyrocarbon layer (40 $\mu \mathrm{m}$ nominal thickness). The kernels were also manufactured by B\&W and identified as kernel lot G73I-14-69307. Two data packages were submitted by $\mathrm{B} \& \mathrm{~W}$ containing the acceptance testing results for the kernels and coated particles, these are identified by their lot numbers. A discussion on the coating of the $\mathrm{B} \& \mathrm{~W}$ TRISO particles can also be found in INL report INL/EXT-09-16545. A data compilation of ORNL analysis of G73J-14-93072A can be found in ORNL/TM-2008/133.

The AGR-2 Fuel Specification (INL SPC-923) provides the requirements necessary for acceptance of the fuel manufactured for the AGR-2 irradiation test. Section 3.3 of SPC-923 provides the property requirements for the heat treated compacts. The Statistical Sampling Plan for AGR-2 Fuel Materials (INL PLN-2691) provides additional guidance regarding statistical methods for product acceptance and recommended sample sizes. The procedures for characterizing and qualifying the compacts are outlined in ORNL product inspection plan AGRCHAR-PIP-12. The inspection report forms generated by this product inspection plan document the product acceptance for the property requirements listed in section 3.3 of SPC-923. Prior to compacting, the overcoated particles are characterized per ORNL product inspection plan AGRCHAR-PIP-11 to obtain data needed for calculation of compacting charge weight and matrix density. Riffling of compact charges is also covered by this procedure. Prior to overcoating, the TRISO particles are characterized per ORNL product inspection plan AGR-CHAR-PIP-10 to determine uranium content, obtain data needed for overcoating and compact fabrication, and obtain further data needed for calculation of matrix density. Riffling of overcoater charges is also covered by this procedure. This document contains all the inspection report forms and data report forms generated by these inspection plans.

In addition to the characterization data, this report also contains other records relevant to the fuel product acceptance. A history of the material flow and sample naming is included. The overcoating and compacting process is summarized. A record of the materials used to make the matrix is also included. A Certificate of Conformance and any applicable Nonconformance Reports are attached as Appendices. 


\section{Table of Contents}

1 Material identification record for LEUO7-OP1-Z compacts __ 5

2 Summary of acceptance test results for LEU07-OP1-Z____ 7

3 Compacting process conditions__ 10

4 Impurity analysis of matrix, resin, and graphites__ 20

5 Characterization of coated particles _ 25

6 Characterization of overcoated particles__ 34

7 Characterization of compacts

Appendix A: Certificate of Conformance __ 116

Appendix B: Nonconformance Reports __ 118 


\section{Material identification record for LEU07-OP1-Z compacts}

Table 1-1 lists the materials used to make the LEU07-OP1-Z compacts, including intermediate batches and samples used for characterization. TRISO-coated particles were shipped from B\&W to ORNL on December 22, 2008. Twenty four completed compacts were shipped to INL on June 24, 2009. Twenty four compacts were retained at ORNL and 107 compacts were consumed at ORNL by the QC acceptance testing. Table 1-2 lists the disposition of each compact.

Table 1-1: Material identification record for LEU07-OP1-Z compacts

\begin{tabular}{|c|c|c|c|}
\hline Sample ID & Parent material & \multicolumn{2}{|l|}{ Notes } \\
\hline G73I-14-69307 & $\begin{array}{l}\text { G73I-14-59370 } \\
\text { G73I-14-59371 } \\
\text { G73I-14-59372 } \\
\text { G73I-14-59373 } \\
\text { G73I-14-59375 }\end{array}$ & \multicolumn{2}{|c|}{ B\&W kernel composite from 5 batches } \\
\hline G73J-14-93072A & G73I-14-69307 & \multicolumn{2}{|c|}{ B\&W TRISO-coated particles } \\
\hline $\begin{array}{l}\text { NP-B8220 } \\
\text { NP-B8221 } \\
\text { NP-B8222 } \\
\text { NP-B8223 } \\
\end{array}$ & G73J-14-93072A & \multicolumn{2}{|c|}{$983 \mathrm{~g}$ sample from 93072A shipped to ORNL on $12 / 22 / 2008$} \\
\hline LEU07 & $\begin{array}{l}\text { NP-B8220 } \\
\text { NP-B8221 } \\
\text { NP-B8222 } \\
\text { NP-B8223 } \\
\end{array}$ & \multicolumn{2}{|c|}{ TRISO-coated particles re-composited after methanol wash } \\
\hline LEU07-A01 & LEU07 & \multicolumn{2}{|c|}{ TRISO-coated particle QC archive } \\
\hline $\begin{array}{l}\text { LEU07-B01 } \\
\text { LEU07-C01 } \\
\text { LEU07-D01 } \\
\text { LEU07-E01 } \\
\text { LEU07-F01 } \\
\text { LEU07-G01 }\end{array}$ & LEU07 & \multicolumn{2}{|c|}{ TRISO-coated particle characterization samples } \\
\hline LEU07-Y\#\# & LEU07 & \multicolumn{2}{|c|}{ Charges for overcoating, numbered Y01 through Y48 } \\
\hline RD13371 & Asbury Graphite Mills & \multicolumn{2}{|l|}{ Natural graphite } \\
\hline KRB2000 & SGL Carbon & \multicolumn{2}{|l|}{ Synthetic graphite } \\
\hline SC1008 & Hexion & \multicolumn{2}{|c|}{ Durite resin lot LK8JD0450 } \\
\hline $\begin{array}{l}\text { RDKrS-011309 } \\
\text { RDKrS-012009 } \\
\text { RDKrS-022009 } \\
\text { RDKrS-021309 }\end{array}$ & $\begin{array}{l}64 \mathrm{wt} \% \text { RD13371 } \\
16 \mathrm{wt} \% \text { KRB2000 } \\
20 \mathrm{wt} \% \text { SC1008 }\end{array}$ & \multicolumn{2}{|c|}{ Matrix precursor batches } \\
\hline LEU07-OP1 & \multicolumn{2}{|c|}{$\begin{array}{l}\text { LEU07-Y01 to LEU07-Y17 + RDKrS-011309 } \\
\text { LEU07-Y18 to LEU07-Y25 + RDKrS-012009 } \\
\text { LEU07-Y27, Y29, Y31 + RDKrS-012009 } \\
\text { LEU07-Y26, Y28, Y30 + RDKrS-022009 } \\
\text { LEU07-Y32 to LEU07-Y44 + RDKrS-022009 } \\
\text { LEU07-Y45 to LEU07-Y48 + RDKrS-021309 }\end{array}$} & Over-coated particle composite \\
\hline LEU07-OP1-A01 & LEU07-OP1 & \multicolumn{2}{|c|}{ Overcoated particle QC archive } \\
\hline $\begin{array}{l}\text { LEU07-OP1-B01 } \\
\text { LEU07-OP1-C01 } \\
\end{array}$ & LEU07-OP1 & \multicolumn{2}{|c|}{ Overcoated particle characterization samples } \\
\hline LEU07-OP1-G\#\#\# & LEU07-OP1 & \multicolumn{2}{|c|}{ Compacts, numbered G001 through G180 } \\
\hline LEU07-OP1-Z\#\#\# & LEU07-OP1-G\#\#\# & \multicolumn{2}{|c|}{$\begin{array}{l}\text { Compacts, numbered Z001 through Z155 } \\
\text { One to one correspondence to G\#\#\# recorded on DRF24C (section 7) }\end{array}$} \\
\hline
\end{tabular}


Table 1-2: Disposition of LEU07-OP1-Z compacts

\begin{tabular}{|c|c|c|c|c|}
\hline Sent to INL & Retained at ORNL & \multicolumn{3}{|c|}{ Consumed during $\mathrm{QC}$ analysis } \\
\hline LEU07-OP1-Z003 & LEU07-OP1-Z008 & LEU07-OP1-Z001 & LEU07-OP1-Z051 & LEU07-OP1-Z107 \\
\hline LEU07-OP1-Z004 & LEU07-OP1-Z015 & LEU07-OP1-Z002 & LEU07-OP1-Z052 & LEU07-OP1-Z108 \\
\hline LEU07-OP1-Z007 & LEU07-OP1-Z016 & LEU07-OP1-Z005 & LEU07-OP1-Z053 & LEU07-OP1-Z110 \\
\hline LEU07-OP1-Z012 & LEU07-OP1-Z036 & LEU07-OP1-Z006 & LEU07-OP1-Z055 & LEU07-OP1-Z111 \\
\hline LEU07-OP1-Z014 & LEU07-OP1-Z054 & LEU07-OP1-Z009 & LEU07-OP1-Z056 & LEU07-OP1-Z112 \\
\hline LEU07-OP1-Z019 & LEU07-OP1-Z063 & LEU07-OP1-Z010 & LEU07-OP1-Z057 & LEU07-OP1-Z114 \\
\hline LEU07-OP1-Z020 & LEU07-OP1-Z065 & LEU07-OP1-Z011 & LEU07-OP1-Z058 & LEU07-OP1-Z115 \\
\hline LEU07-OP1-Z021 & LEU07-OP1-Z067 & LEU07-OP1-Z013 & LEU07-OP1-Z059 & LEU07-OP1-Z119 \\
\hline LEU07-OP1-Z041 & LEU07-OP1-Z071 & LEU07-OP1-Z017 & LEU07-OP1-Z060 & LEU07-OP1-Z120 \\
\hline LEU07-OP1-Z043 & LEU07-OP1-Z073 & LEU07-OP1-Z018 & LEU07-OP1-Z061 & LEU07-OP1-Z122 \\
\hline LEU07-OP1-Z068 & LEU07-OP1-Z084 & LEU07-OP1-Z022 & LEU07-OP1-Z062 & LEU07-OP1-Z123 \\
\hline LEU07-OP1-Z072 & LEU07-OP1-Z089 & LEU07-OP1-Z023 & LEU07-OP1-Z064 & LEU07-OP1-Z124 \\
\hline LEU07-OP1-Z074 & LEU07-OP1-Z098 & LEU07-OP1-Z024 & LEU07-OP1-Z066 & LEU07-OP1-Z125 \\
\hline LEU07-OP1-Z080 & LEU07-OP1-Z102 & LEU07-OP1-Z025 & LEU07-OP1-Z069 & LEU07-OP1-Z127 \\
\hline LEU07-OP1-Z081 & LEU07-OP1-Z109 & LEU07-OP1-Z026 & LEU07-OP1-Z070 & LEU07-OP1-Z129 \\
\hline LEU07-OP1-Z082 & LEU07-OP1-Z113 & LEU07-OP1-Z027 & LEU07-OP1-Z075 & LEU07-OP1-Z130 \\
\hline LEU07-OP1-Z093 & LEU07-OP1-Z116 & LEU07-OP1-Z028 & LEU07-OP1-Z076 & LEU07-OP1-Z133 \\
\hline LEU07-OP1-Z094 & LEU07-OP1-Z126 & LEU07-OP1-Z029 & LEU07-OP1-Z077 & LEU07-OP1-Z134 \\
\hline LEU07-OP1-Z105 & LEU07-OP1-Z131 & LEU07-OP1-Z030 & LEU07-OP1-Z078 & LEU07-OP1-Z135 \\
\hline LEU07-OP1-Z106 & LEU07-OP1-Z132 & LEU07-OP1-Z031 & LEU07-OP1-Z079 & LEU07-OP1-Z136 \\
\hline LEU07-OP1-Z117 & LEU07-OP1-Z137 & LEU07-OP1-Z032 & LEU07-OP1-Z083 & LEU07-OP1-Z138 \\
\hline LEU07-OP1-Z118 & LEU07-OP1-Z143 & LEU07-OP1-Z033 & LEU07-OP1-Z085 & LEU07-OP1-Z139 \\
\hline LEU07-OP1-Z121 & LEU07-OP1-Z148 & LEU07-OP1-Z034 & LEU07-OP1-Z086 & LEU07-OP1-Z140 \\
\hline LEU07-OP1-Z128 & LEU07-OP1-Z151 & LEU07-OP1-Z035 & LEU07-OP1-Z087 & LEU07-OP1-Z141 \\
\hline & & LEU07-OP1-Z037 & LEU07-OP1-Z088 & LEU07-OP1-Z142 \\
\hline & & LEU07-OP1-Z038 & LEU07-OP1-Z090 & LEU07-OP1-Z144 \\
\hline & & LEU07-OP1-Z039 & LEU07-OP1-Z091 & LEU07-OP1-Z145 \\
\hline & & LEU07-OP1-Z040 & LEU07-OP1-Z092 & LEU07-OP1-Z146 \\
\hline & & LEU07-OP1-Z042 & LEU07-OP1-Z095 & LEU07-OP1-Z147 \\
\hline & & LEU07-OP1-Z044 & LEU07-OP1-Z096 & LEU07-OP1-Z149 \\
\hline & & LEU07-OP1-Z045 & LEU07-OP1-Z097 & LEU07-OP1-Z150 \\
\hline & & LEU07-OP1-Z046 & LEU07-OP1-Z099 & LEU07-OP1-Z152 \\
\hline & & LEU07-OP1-Z047 & LEU07-OP1-Z100 & LEU07-OP1-Z153 \\
\hline & & LEU07-OP1-Z048 & LEU07-OP1-Z101 & LEU07-OP1-Z154 \\
\hline & & LEU07-OP1-Z049 & LEU07-OP1-Z103 & LEU07-OP1-Z155 \\
\hline & & LEU07-OP1-Z050 & LEU07-OP1-Z104 & \\
\hline
\end{tabular}




\section{Summary of acceptance test results for LEU07-OP1-Z}

At the end of this section is the inspection report form IRF-12A associated with the compact lot LEU06-OP1-Z. This inspection report form also appears in section 7 of this compilation, accompanied by the associated data report forms (DRFs) showing the results of each individual measurement. The inspection report form summarizes the acceptance testing performed according to the product inspection plan AGR-CHAR-PIP-12. The information in this form covers all the property specifications listed in section 3.3 of the AGR-2 Fuel Specification (INL SPC-923, Rev. 3). The compact lot, LEU07-OP1-Z, did not meet all the requirements in section 3.3 of SPC-923, Rev. 3. A nonconformance related to compact length was determined to be acceptable. However, a higher than allowed fraction of exposed uranium was determined to not be acceptable for the AGR-2 irradiation test. The exposed uranium was due to cracked TRISO layers in the coated particle composite. These cracks are thought to have occurred when particles were removed from the coating furnace using a suction device. In addition, a higher than allowed IPyC defect fraction was determined to not be acceptable for the AGR-2 irradiation test. Excessive permeability of chlorine through the IPyC during $\mathrm{SiC}$ deposition was evident from $\mathrm{x}-$ ray observation of uranium dispersion out of the kernel and into the buffer after particles experienced $1800^{\circ} \mathrm{C}$ heat treatment in the final stage of compacting. The final disposition of this compact lot was to not use the compacts for the AGR-2 irradiation test, but to retain the compacts in storage at ORNL and INL for possible future analysis or methods development. This disposition was documented on INL NCR-44791 and INL NCR-44792.

Table 2-1 is provided for quick reference. It gives the mean values of key variable properties of the compact lot, LEU07-OP1-Z. For standard deviations of the distribution of the measured values see the appropriate IRF or DRF. For discussions on the uncertainty in these values, see the associated data acquisition methods and data report forms.

Table 2-1: Quick reference table for key variable properties of LEU07-OP1-Z.

\begin{tabular}{|l|c|}
\hline \multicolumn{1}{|c|}{ Property } & Mean \\
\hline Mean uranium loading (g U/compact) & 1.251 \\
\hline Compact diameter $(\mathrm{mm})$ & 12.32 \\
\hline Compact length $(\mathrm{mm})$ & 25.19 \\
\hline Compact mass $(\mathrm{g})$ & 6.346 \\
\hline Compact matrix density $\left(\mathrm{g} / \mathrm{cm}^{3}\right)$ & 1.61 \\
\hline Impurity content & \\
\hline
\end{tabular}

The reported mean impurity levels for the fuel compacts, recorded on IRF-12A and IRF-12B, may be higher than the actual values. This is because the as-reported mean impurity levels do not reflect the fact that some of the measurements were at or below the mass spectrometry measurement threshold, and thus could not be differentiated from zero. For the purpose of the acceptance test, impurity values reported as threshold values (documented in the data report forms with the < symbol) are always assumed to be equal to the maximum possible value. In addition, each time a leach was performed, a blank run was also performed, where all the relevant wet chemistry steps in the leach-burn-leach procedure in AGR-CHAR-DAM-26R1 were 
performed without a compact present, in order to obtain background values for each analyzed impurity. If a measurable impurity value was obtained in the blank, then that value was subtracted from the measured value in each sample. However, if a threshold value was reported in the blank, then no background subtraction was performed. Table 2-2 shows the possible range for the measured impurities, where the upper limit is the as-reported mean and the lower limit is the possible minimum value calculated by accounting for the fact that values reported as threshold values could have been as low as zero. This range reflects the uncertainty in the measured impurity values due to the mass spectrometry measurement thresholds.

Table 2-2: Mean impurity levels for fuel compacts from LEU07-OP1-Z compact lot measured by deconsolidation leach-burn-leach technique.

\begin{tabular}{|c|c|}
\hline Impurity & Measured impurity content $(\boldsymbol{\mu g}$ /compact) \\
\hline Iron & $0.70-4.78$ \\
\hline Chromium & $0.37-0.59$ \\
\hline Manganese & $0.00-0.20$ \\
\hline Cobalt & $0.00-0.16$ \\
\hline Nickel & $0.02-0.84$ \\
\hline Calcium & $30.35-36.05$ \\
\hline Aluminum & $30.01-30.08$ \\
\hline Titanium & $2.17-3.05$ \\
\hline Vanadium & $18.01-18.23$ \\
\hline
\end{tabular}

Table 2-3 is also provided for quick reference. It gives the upper limit of the $95 \%$ confidence interval of the defect fraction for key attribute properties of the compact lot LEU07-OP1-Z. In other words, these values are the lowest tolerance limits for which the compact lot would be deemed acceptable at $95 \%$ confidence, based on the particular sample that was measured. Also listed in the table are the actual number of defects observed and the number of particles analyzed. Note that in the case of all but the uranium contamination fraction, zero defects were observed. The defect fractions listed in the table for these cases are limited by the number of particles measured and the actual defect fraction could be much lower.

Table 2-3: Quick reference table for key attribute properties of LEU07-OP1-Z.

\begin{tabular}{|l|c|c|}
\hline \multicolumn{1}{|c|}{ Property } & $\begin{array}{c}\text { Observed Number of Defects/ } \\
\text { Number of Particles Analyzed }\end{array}$ & $\begin{array}{c}\text { 95\% Confidence } \\
\text { Defect Fraction }\end{array}$ \\
\hline \hline Uranium contamination fraction & $15 / 317690$ & $\leq 7.3 \mathrm{E}-5$ \\
\hline Defective SiC coating fraction & $0 / 190614$ & $\leq 1.6 \mathrm{E}-5$ \\
\hline Defective IPyC coating fraction & $443 / 63538$ & $\leq 7.6 \mathrm{E}-3$ \\
\hline Defective OPyC coating fraction & $0 / 3177$ & $\leq 9.5 \mathrm{E}-4$ \\
\hline
\end{tabular}

It is also interesting to note the increase in pyrocarbon anisotropy due to compact heat treatment. The diattenuation of the IPyC increased from $0.0118 \pm 0.0006$ to $0.0150 \pm 0.0006(1.0355 \pm 0.0019$ to $1.0451 \pm 0.0019$ in terms of effective $\mathrm{BAFo})$. The diattenuation of the $\mathrm{OPyC}$ increased from $0.0081 \pm 0.0003$ to $0.0129 \pm 0.0007(1.0242 \pm 0.0008$ to $1.0388 \pm 0.0021$ in terms of effective BAFo). 
Inspection Report Form IRF-12A: AGR-2 UCO Fuel Compact Lots

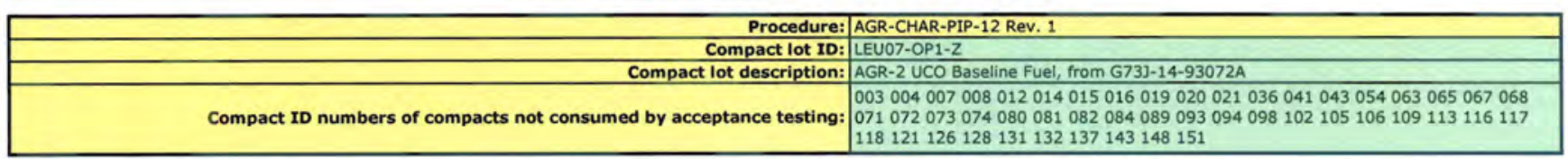

\begin{tabular}{|c|c|c|c|c|c|c|c|c|c|}
\hline \multirow[b]{2}{*}{ Property } & \multicolumn{4}{|c|}{ Measured Data } & Specification & \multirow[b]{2}{*}{ Acceptance Criteria } & \multirow{2}{*}{$\begin{array}{l}\text { Acceptance } \\
\text { Test Value }\end{array}$} & \multirow{2}{*}{$\begin{array}{c}\text { Pass } \\
\text { or } \\
\text { fail } \\
\end{array}$} & \multirow{2}{*}{$\begin{array}{c}\text { Data } \\
\text { Records }\end{array}$} \\
\hline & $\begin{array}{c}\text { Mean } \\
(x)\end{array}$ & $\begin{array}{c}\text { Std. Dev. } \\
(\mathbf{s})\end{array}$ & $\begin{array}{c}\text { Measurements } \\
(\mathrm{n})\end{array}$ & $\begin{array}{l}k \text { or } t \\
\text { value }\end{array}$ & $\begin{array}{l}\text { INL SPC-923 } \\
\text { Revison } 3 \\
\end{array}$ & & & & \\
\hline \multirow{2}{*}{$\begin{array}{l}\text { Uranium loading } \\
\text { (gU/compact) }\end{array}$} & \multirow{2}{*}{1.251} & \multirow{2}{*}{0.005} & \multirow{2}{*}{6} & \multirow{2}{*}{2.015} & \multirow{2}{*}{$1.265 \pm 0.07$} & $A=x-t s / \sqrt{ } n \geq 1.195$ & 1.247 & pass & \multirow{2}{*}{ DRF-25 } \\
\hline & & & & & & $B=x+t s / \sqrt{n} \leq 1.335$ & 1.255 & pass & \\
\hline Compact diameter (mm) & \multirow{3}{*}{\multicolumn{2}{|c|}{ See DRF-24 }} & & & $12.22-12.46$ & \multirow{3}{*}{$\begin{array}{l}\text { All available for irradiation test } \\
\text { meet specification }\end{array}$} & \multirow{3}{*}{ See DRF-24 } & fail & \multirow{3}{*}{ DRF-24 } \\
\hline Compact length $(\mathrm{mm})$ & & & & & $25.02-25.40$ & & & & \\
\hline Compact matrix density $\left(\mathrm{g} / \mathrm{cm}^{3}\right)$ & & & & & $\geq 1.45$ & & & pass & \\
\hline \multirow{2}{*}{$\begin{array}{l}\text { Iron content outside SiC } \\
\qquad(\mu \mathrm{g} / \mathrm{compact})\end{array}$} & \multirow{2}{*}{4.78} & \multirow{2}{*}{2.17} & \multirow{2}{*}{12} & 1.796 & $\begin{array}{l}\text { mean } \\
\leq 25\end{array}$ & $B=x+t s / \sqrt{n} \leq 25$ & 5.9 & pass & \multirow{2}{*}{$\begin{array}{l}\text { IRF-12B } \\
\text { DRF-26 }\end{array}$} \\
\hline & & & & 3.747 & $\begin{array}{c}\text { dispersion } \\
\leq 0.01 \geq 100 \\
\end{array}$ & $D=x+\sqrt{3} k s<100$ & 18.9 & pass & \\
\hline $\begin{array}{l}\text { Chromium content outside SiC } \\
\text { ( } \mu \mathrm{g} / \text { compact })\end{array}$ & 0.59 & 0.06 & 12 & 1.796 & $\begin{array}{l}\text { mean } \\
\leq 50\end{array}$ & $B=x+t s / \sqrt{n} \leq 50$ & 0.6 & pass & $\begin{array}{l}\text { IRF-12B } \\
\text { DRF-26 } \\
\end{array}$ \\
\hline $\begin{array}{c}\text { Manganese content outside SiC } \\
(\mu \mathrm{g} / \text { compact })\end{array}$ & 0.20 & 0.09 & 12 & 1.796 & $\begin{array}{l}\text { mean } \\
\leq 50\end{array}$ & $B=x+t s / \sqrt{ } n \leq 50$ & 0.2 & pass & $\begin{array}{l}\text { IRF-12B } \\
\text { DRF-26 }\end{array}$ \\
\hline $\begin{array}{c}\text { Cobalt content outside SiC } \\
(\mu \mathrm{g} / \text { compact })\end{array}$ & 0.16 & 0.07 & 12 & 1.796 & $\begin{array}{l}\text { mean } \\
\leq 50\end{array}$ & $B=x+t s / \sqrt{n} \leq 50$ & 0.2 & pass & $\begin{array}{l}\text { IRF-12B } \\
\text { DRF-26 }\end{array}$ \\
\hline $\begin{array}{c}\text { Nickel content outside SiC } \\
\text { ( } \mu \mathrm{g} / \text { compact) }\end{array}$ & 0.84 & 0.36 & 12 & 1.796 & $\begin{array}{l}\text { mean } \\
\leq 50\end{array}$ & $B=x+t s / \sqrt{n} \leq 50$ & 1.0 & pass & $\begin{array}{l}\text { IRF-12B } \\
\text { DRF-26 } \\
\end{array}$ \\
\hline $\begin{array}{c}\mathrm{Cr}+\mathrm{Mn}+\mathrm{Co}+\mathrm{Ni} \text { content outside } \mathrm{SiC} \\
(\mu \mathrm{g} / \text { compact })\end{array}$ & 1.78 & 0.50 & 12 & 3.747 & $\begin{array}{c}\text { dispersion } \\
\leq 0.01 \geq 200 \\
\end{array}$ & $D=x+\sqrt{ } 3 k s<200$ & 5.0 & pass & $\begin{array}{l}\text { IRF-12B } \\
\text { DRF-26 }\end{array}$ \\
\hline $\begin{array}{l}\text { Calcium content outside SiC } \\
\text { ( } \mu \mathrm{g} / \text { compact) }\end{array}$ & 36.05 & 6.22 & 12 & 1.796 & $\begin{array}{l}\text { mean } \\
\leq 50\end{array}$ & $B=x+t s / \sqrt{n} \leq 50$ & 39.3 & pass & $\begin{array}{l}\text { IRF-12B } \\
\text { DRF-26 }\end{array}$ \\
\hline $\begin{array}{l}\text { Aluminum content outside SiC } \\
\text { ( } \mu \mathrm{g} / \text { compact) }\end{array}$ & 30.08 & 9.54 & 12 & 1.796 & $\begin{array}{l}\text { mean } \\
\leq 50 \\
\end{array}$ & $B=x+t s / \sqrt{n} \leq 50$ & 35.0 & pass & $\begin{array}{l}\text { IRF-12B } \\
\text { DRF-26 } \\
\end{array}$ \\
\hline $\begin{array}{c}\mathrm{Ti}+\mathrm{V}_{(\mu \mathrm{g} / \text { compact })} \\
\text { content outside SiC }\end{array}$ & 21.28 & 1.04 & 12 & 1.796 & $\begin{array}{l}\text { mean } \\
\leq 240\end{array}$ & $B=x+t s / \sqrt{n} \leq 240$ & 21.8 & pass & $\begin{array}{l}\text { IRF-12B } \\
\text { DRF-26 }\end{array}$ \\
\hline
\end{tabular}

\begin{tabular}{|c|c|c|c|c|c|c|c|}
\hline \multirow[b]{2}{*}{ Property } & \multicolumn{2}{|c|}{ Measured Data } & \multirow{2}{*}{$\begin{array}{c}\text { Specification } \\
\text { INL SPC-923 } \\
\text { Revison } 2 \\
\end{array}$} & \multirow[b]{2}{*}{ Acceptance Criteria } & \multirow[b]{2}{*}{$\begin{array}{l}\text { Acceptance } \\
\text { Test Value }\end{array}$} & \multirow{2}{*}{$\begin{array}{c}\text { Pass } \\
\text { or } \\
\text { fail } \\
\end{array}$} & \multirow[b]{2}{*}{$\begin{array}{c}\text { Data } \\
\text { Records }\end{array}$} \\
\hline & $\begin{array}{c}\text { \# of } \\
\text { compacts }\end{array}$ & $\begin{array}{c}\text { \# of } \\
\text { particles }\end{array}$ & & & & & \\
\hline $\begin{array}{l}\text { Uranium contamination fraction } \\
\text { ( } g \text { exposed } U / \text { gram } U \text { in compact) }\end{array}$ & 100 & 317690 & $\leq 2.0 \times 10^{-5}$ & $\begin{array}{c}\leq 1 \text { effectively exposed kernel in } \geq 237192 \text { particles } \\
\text { or } \leq 2 \text { effectively exposed kernels in } \geq 314788 \text { particles } \\
\end{array}$ & 14.6 & fail & $\begin{array}{l}\text { IRF-12C } \\
\text { DRF-26 } \\
\end{array}$ \\
\hline $\begin{array}{l}\text { Defective SiC coating fraction } \\
\text { (fraction of total particles) }\end{array}$ & 60 & 190614 & $\leq 1.0 \times 10^{-4}$ & $\begin{array}{c}\leq 2 \text { leached kernels in } \geq 62956 \text { particles } \\
\text { or } \leq 6 \text { leached kernels in } \geq 118422 \text { particles }\end{array}$ & 0 & pass & $\begin{array}{l}\text { IRF-12D } \\
\text { DRF-26 }\end{array}$ \\
\hline $\begin{array}{l}\text { Defective IPyC coating fraction } \\
\text { (fraction of total particles) }\end{array}$ & 20 & 63538 & $\leq 1.0 \times 10^{-4}$ & $\begin{array}{l}\leq 1 \text { with excessive } U \text { dispersion in } \geq 47437 \text { particles } \\
\text { or } \leq 2 \text { with excessive } U \text { dispersion in } \geq 62956 \text { particles }\end{array}$ & 443 & fall & DRF-28 \\
\hline $\begin{array}{l}\text { Defective OPyC coating fraction } \\
\text { (fraction of total particles) }\end{array}$ & 1 & 3177 & $\leq 0.01$ & $\leq 6$ cracked or missing OPyC in $\geq 1182$ particles & 0 & pass & DRF-27 \\
\hline
\end{tabular}

\section{Comments}

One compact was outside of the length specification by $+0.046 \mathrm{~mm}$. This non-conformance was documented on ORNL NCR-X-MSTD-AGR-10-01 with a disposition of use as is.

A $15 / 317690$ uranium contamination fraction is above the specified limit and corresponds to $<7.3 \mathrm{e}-5$ at $95 \%$ confidence. This non-conformance was documented on INL NCR-44791 with a disposition of do not use for AGR-2 irradiation test, but retain for other uses.

A $443 / 63538$ defective IPYC coating fraction is above the specified limit and corresponds to $<7.6 \mathrm{e}-3$ at $95 \%$ confidence. This non-conformance was documented on INL NCR-44792 with a

disposition of do not use for AGR-2 irradiation test, but retain for other uses.
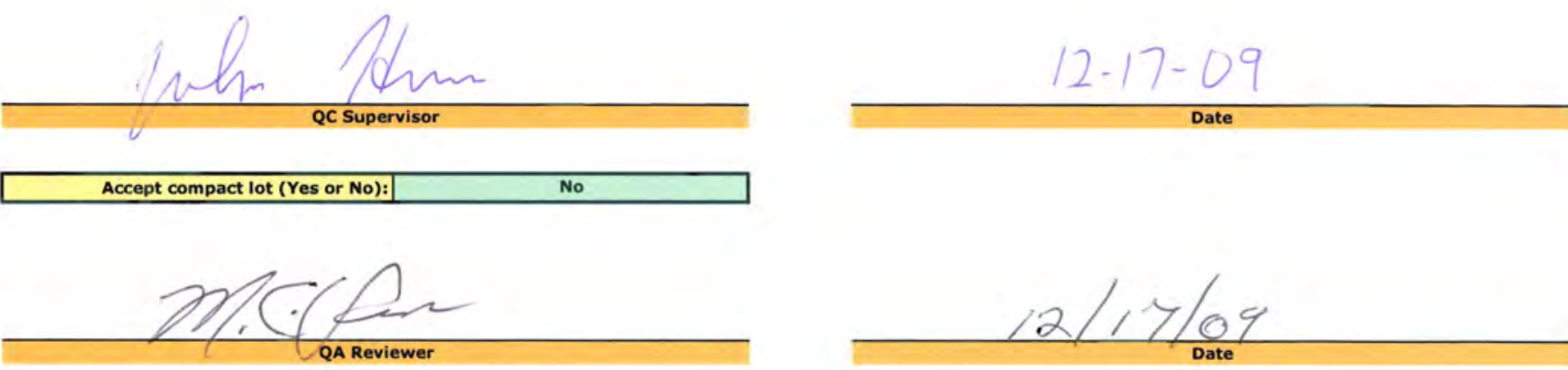


\section{Compacting process conditions}

Four samples totaling $983 \mathrm{~g}$ were riffled from coated particle batch G73J-14-93072A using a chute splitter, and shipped from B\&W on December 22, 2008. After receipt, the particles were washed in methanol per procedure AGR-TRISOWASH-SOP-1, "Standard Operating Procedure for TRISO Particle Washing." Washing of particles prior to overcoating was adapted in order to help reduce the amount of contamination on the particles that may have been acquired during processing or general handling. This procedure also reduces the amount of loose carbon dust on the surface of the particles. The washing procedure was adopted from General Atomics' particle washing procedures.

After washing, particles were combined and homogenized and renamed LEU07 and AGRCHAR-PIP-10, Rev. 2, "Product Inspection Plan for AGR-2 Particles for Compacting Preliminary Measurements" was completed. This plan calls for measurement of average particle weight, diameter, envelope volume, and uranium content. The plan also calls for riffling of 20 gram aliquots for use as overcoater charges. Riffling at ORNL was done using a 10 position rotary riffler. After riffling out the characterization samples, forty-eight overcoater charges were prepared and labeled LEU07-Y01 through Y48. The results of the PIP-10 inspection are reported in section 5. Additional ORNL characterization performed on another sample taken from G73J14-93072A is provided in ORNL/TM-2008/133, "Data Compilation for AGR-2 UCO Baseline Coated Particle Batch G73J-14-93072A".

One $\sim 20 \mathrm{~g}$ aliquot was used per overcoating run. Overcoating was performed according to AGRCOMP-SOP-2, Rev. 1, "Standard Operating Procedure for Overcoating TRISO Particles." The LEU07-Y\#\# riffled aliquots were overcoated with the following matrix batches: Y01-Y17 (RDKrS 011309), Y18-Y25, Y27, Y29, Y31 (RDKrS 012009), Y26, Y28, Y30, Y32-Y44 (RDKrS 022009), and Y45-Y48 (RDKrS 021309).

In total, 1396 grams of $-12 /+16$ overcoated particles were produced by overcoating TRISO aliquots Y01-Y48. "-12/+16" overcoated particles are those that pass through an ASTM E11 No. 12 sieve (1.70 mm nominal opening) but do not pass through an ASTM E11 No. 16 sieve (1.18 mm nominal opening). The 1396 grams of sieved overcoated particles was tabled and 1214 grams of Bin 3 particles were recovered. "Bin 3" particles are those particles that end up in the third bin of a shape separation inclined table; these are the most spherical overcoated particles. 1214 grams of Bin 3 overcoated particles was determined to be a sufficient quantity to produce at least 180 compacts, based on preliminary calculations. The total number of compacts required for acceptance testing, irradiation, and spares was 155 .

After overcoating, the overcoated particles from Y01-Y48 were combined and homogenized into an overcoated particle composite. The overcoated particle composite was labeled LEU07-OP1 and AGR-CHAR-PIP-11, Rev. 0, "Product Inspection Plan for AGR-2 Overcoated Particles for Compacting" was completed. This plan calls for measurement of average overcoated particle weight and diameter. The results of the PIP-11 inspection are reported in section 7. The plan also calls for riffling of compact charges for pressing. 
Based on the average uranium loading determined for the LEU07 particles of $3.930 \cdot 10^{-4} \mathrm{~g}$ (section 5), 3219 particles would be needed in each compact to obtain an average uranium loading of $1.265 \mathrm{~g}$ for the compacts (the specified loading in SPC-923 was $1.265 \pm 0.07 \mathrm{~g}$ ). However, for consistency with the LEU06-OP1-Z compact lot (see LEU06-OP1-Z compacts data package ORNL/TM-2009/304) a target of 3200 particles per compact was chosen. This compact charge was based on the measured average uranium loading for LEU06 particles of $3.953 \cdot 10^{-4} \mathrm{~g}$. Note that both of these particle batches used the same kernel charge so the uranium loading was expected to be the same. The average LEU07-OP1 overcoated particle weight was measured to be $2.018 \cdot 10^{-3} \mathrm{~g}$ (section 6). Using this value, a compact charge of $6.458 \mathrm{~g}$ was calculated in order to achieve a compact with a uranium loading of $1.265 \pm 0.07 \mathrm{~g}$. One hundred and eighty compact charges were prepared and labeled LEU07-OP1-G001 through G180. A record of the weight of each compact charge can be found on data report form DRF-24D, in section 7.

Actual compact uranium loading was measured to be $1.251 \pm 0.005 \mathrm{~g}$. Twenty compacts were deconsolidated and the particles were counted as part of the x-ray analysis for possible uranium dispersion due to defective IPyC. The average number of particles per compact was determined to be 3177,23 particles short of the target compact loading. This reduced number of particles per compact explains the slightly low uranium loading result. It is hypothesized that the reason for the undershoot in particles per compact was due to weight loss from the overcoated particles from evaporation of methanol and volatiles from the resin. Overcoated particles were kept in sealed containers as much as feasible during riffling of the compact charges. However, it is likely that the weight loss due to evaporation for the samples used to determine average particle weight was greater than for the overcoated particles weighed out into each compact charge. Therefore, the average overcoated particle weight used to calculate the target compact charge was slightly too low.

Note that all the uranium per particle measurements on the LEU06 and LEU07 material were consistent to within the sampling error and measurement uncertainty, as expected given that the same kernel batch was used for both coating runs. Table 3-1 shows the measured average uranium content in the particles from the uranium analyses performed directly on the particles compared to that calculated from the analyses performed on the compacts divided by the average number of particles per compact.

Table 3-1: Average and standard deviation ( \pm value) of four independent measurements of uranium per particle for LEU06 and LEU07 particles.

\begin{tabular}{|l|c|c|}
\hline & LEU06 & LEU07 \\
\hline \hline grams U/particle based on particle analysis & $3.953 \pm 0.010 \cdot 10^{-4} \mathrm{~g}$ & $3.930 \pm 0.007 \cdot 10^{-4} \mathrm{~g}$ \\
\hline grams U/particle based on compact analysis & $3.942 \pm 0.006 \cdot 10^{-4} \mathrm{~g}$ & $3.938 \pm 0.016 \cdot 10^{-4} \mathrm{~g}$ \\
\hline
\end{tabular}

The LEU07-OP1-G\#\# compacting charges were formed into green compacts using a heated, double acting die and a Carver hydraulic press. Compacting was performed in accordance with AGR-COMP-SOP-3, Rev. 1, "Standard Operating Procedure for Compacting". The die was heated to $70^{\circ} \mathrm{C}$ and approximately $0.10 \mathrm{~g}$ of matrix was added to the top and bottom of the compact in order to create matrix "end caps." The end caps were formed with the compact by 
first pouring a matrix charge into the heated die, followed by the overcoated particles, and then a second charge of matrix. This forming method created a thin (less than $0.5 \mathrm{~mm}$ thick) fuel free zone on the ends of the compact, called end caps. In total, 180 green compacts were fabricated. The compacts retained the designation of the riffled charges, LEU07-OP1-G001 through G180. All 180 green compacts were carbonized and heat treated according to AGR-COMP-SOP-4, Rev. 0, "Standard Operating Procedure for Carbonizing Compacts," and AGR-COMP-SOP-5 Rev. 1, "Standard Operating Procedure for Heat-treating Compacts."

Prior to compacting the LEU07-OP1-G\#\#\# charges, test compacts were made using excess material from the Bin 2 overcoated particles that were accumulated after tabling. Bin 2 overcoated particles are spherical in nature, but not as spherical as the Bin 3 overcoated particles that were retained for the LEU07-OP1 composite. LEU07 Bin 2 overcoated particles were compacted in an effort to determine the optimal pressing temperature needed to avoid end cap fissures. End cap fissures were found in some of the LEU06 compacts and were evidenced by what appeared to be a thin surface crack where the end caps met the overcoated particles. The end cap fissures appeared to be caused by adhesion of the end caps to the pressing rams at the elevated temperatures used for LEU06-OP1-Z compacting. AGR-1 compacts were made at room temperature with a higher methanol content in the overcoat in order to reduce the compacting pressure. The AGR-2 compact fabrication process replaced the high methanol content with the use of an elevated compacting temperature to help the overcoat to flow easier. Compacting at elevated temperature was more in line with the current AGR program plans for future compact scale-up, which may use elevated temperature instead of methanol to enhance resin flow and which may involve temperatures as high as $140^{\circ} \mathrm{C}$, depending on the type of resin used. Note that the end cap fissure defect didn't appear during earlier AGR-2 compacting development efforts because the defect fraction was too low to observe until a large number of compacts were processed under identical conditions. Minor modifications to the compacting process parameters were explored in order to eliminate the presence of end cap fissures. In total, 28 LEU07 Bin 2 test compacts were made at pressing temperatures ranging from 45 to $95^{\circ} \mathrm{C}$. It was found that no fissures were observed at lower temperatures, but that greater than 700 lbs-f $(3100 \mathrm{~N})$ pressing forces were needed to achieve target packing fractions and matrix densities. The optimal pressing temperature and subsequent force was found to be a pressing temperature of $70^{\circ} \mathrm{C}$, which required a force of $\sim 560 \mathrm{lbs}-\mathrm{f}(\sim 2500 \mathrm{~N})$. This was a lower temperature and higher pressure than used for LEU06-OP1-Z compacts $\left(94.5^{\circ} \mathrm{C}, \sim 2000 \mathrm{~N}\right)$.

After compacting, 155 compacts were selected from LEU07-OP1-G001 through G180 for use. Compacts with obvious processing defects, chips, or undesirable dimensions were sorted out and not included in the 155 compacts selected for the final fuel compact lot. This down-select was part of the compacting process and was performed prior to random selection of compacts for acceptance testing. It should be understood that the results in this section and the acceptance testing are only relevant for the final 155 compact lot from which random representative samples were drawn for characterization. As instructed in AGR-CHAR-PIP-12, Rev. 1, "Product Inspection Plan for AGR-2 UCO Fuel Compact Lots", these 155 compacts were randomized and relabeled as LEU07-OP1-Z001 through Z155. A record of the original G-number for each Znumbered compact can be found on data report form DRF-24C, in section 7. After relabeling, the compacts were characterized for product acceptance according to product inspection plan PIP12. This plan calls for measurement of compact length, diameter, mass, matrix density, uranium 
content, impurity content, and determination of defect fractions for exposed uranium, defective $\mathrm{SiC}$, uranium dispersion due to defective IPyC, and defective OPyC.

\section{AGR-2 Process Conditions}

The LEU07-OP1-Z (AGR-2 UCO Baseline) compact lot was made in accordance with the AGR2 Fuel Specification (SPC-923, Rev. 3). The specified AGR-2 process limits are listed below.

Molding Pressure: $\quad<60 \mathrm{MPa}$

Carbonization parameters: $\quad<350^{\circ} \mathrm{C} / \mathrm{hr}$ in $\mathrm{He}$ atmosphere

Hold at $950 \pm 50^{\circ} \mathrm{C}$ for $1.0 \pm 0.4 \mathrm{hr}$

Furnace cool

Heat treatment parameters: $\quad 220^{\circ} \mathrm{C} / \mathrm{min}$ in vacuum $(<1.3 \mathrm{~Pa})$

Hold at $1650-1850^{\circ} \mathrm{C}$ for $60 \pm 10 \mathrm{~min}$

Furnace cool at $\sim 20^{\circ} \mathrm{C} / \mathrm{min}$ to below $700^{\circ} \mathrm{C}$

Table 3-2 shows the process conditions used in molding the compacts, carbonizing the compacts, and heat treating the compacts. In the carbonization regime, the furnace was allowed to cool under no power (i.e., after holding at $950^{\circ} \mathrm{C}$ for 1 hour, power was turned off). In the heat treatment run, the furnace was cooled under power at $20^{\circ} \mathrm{C} / \mathrm{min}$ until the furnace temperature reached $700^{\circ} \mathrm{C}$, and then the furnace was allowed to cool under no power. 
Table 3-2: Summary of process conditions used in making LEU07-OP1-Z (AGR-2 UCO Baseline) compacts

\begin{tabular}{|c|c|c|c|c|c|c|c|c|c|}
\hline \multirow{2}{*}{ Compact ID } & \multicolumn{5}{|c|}{ Carbonization Parameter } & \multicolumn{4}{|c|}{ Heat-treatment Parameters } \\
\hline & $\begin{array}{l}\text { Molding Pressure } \\
(\mathrm{MPa})\end{array}$ & $\begin{array}{l}\text { Heating Rate } \\
\left({ }^{\circ} \mathrm{C} / \mathrm{min} .\right)\end{array}$ & $\begin{array}{l}\text { Max. Temp. } \\
\left({ }^{\circ} \mathrm{C}\right)\end{array}$ & $\begin{array}{l}\text { Hold Time } \\
\text { (hrs.) }\end{array}$ & Atmosphere & $\begin{array}{c}\text { Heating Rate } \\
\left({ }^{\circ} \mathrm{C} / \mathrm{min} .\right)\end{array}$ & $\begin{array}{l}\text { Max. Temp. } \\
\left({ }^{\circ} \mathrm{C}\right)\end{array}$ & $\begin{array}{l}\text { Hold Time } \\
\text { (hrs.) }\end{array}$ & Atmosphere \\
\hline LEU07-OP1-Z001 & 20.9 & 4.7 & 950 & 1 & flowing $\mathrm{He}$ & 20 & 1800 & 1 & vacuum \\
\hline LEU07-OP1-Z002 & 20.9 & 4.7 & 950 & 1 & flowing $\mathrm{He}$ & 20 & 1800 & 1 & vacuum \\
\hline LEU07-OPI-Z003 & 20.9 & 4.7 & 950 & 1 & flowing $\mathrm{He}$ & 20 & 1800 & 1 & vacuum \\
\hline LEU07-OP1-Z004 & 20.9 & 4.7 & 950 & 1 & flowing $\mathrm{He}$ & 20 & 1800 & 1 & vacuum \\
\hline LEU07-OP1-Z005 & 20.9 & 4.7 & 950 & 1 & flowing $\mathrm{He}$ & 20 & 1800 & 1 & vacuum \\
\hline LEU07-OP1-Z006 & 20.9 & 4.7 & 950 & 1 & flowing $\mathrm{He}$ & 20 & 1800 & 1 & vacuum \\
\hline LEU07-OP1-Z007 & 20.9 & 4.7 & 950 & 1 & flowing $\mathrm{He}$ & 20 & 1800 & 1 & vacuum \\
\hline LEU07-OP1-Z008 & 20.9 & 4.7 & 950 & 1 & flowing $\mathrm{He}$ & 20 & 1800 & 1 & vacuum \\
\hline LEU07-OP1-Z009 & 20.9 & 4.7 & 950 & 1 & flowing $\mathrm{He}$ & 20 & 1800 & 1 & vacuum \\
\hline LEU07-OP1-Z010 & 20.9 & 4.7 & 950 & 1 & flowing $\mathrm{He}$ & 20 & 1800 & 1 & vacuum \\
\hline LEU07-OP1-Z011 & 21.7 & 4.7 & 950 & 1 & flowing $\mathrm{He}$ & 20 & 1800 & 1 & vacuum \\
\hline LEU07-OP1-Z012 & 20.9 & 4.7 & 950 & 1 & flowing $\mathrm{He}$ & 20 & 1800 & 1 & vacuum \\
\hline LEU07-OP1-Z013 & 20.9 & 4.7 & 950 & 1 & flowing $\mathrm{He}$ & 20 & 1800 & 1 & vacuum \\
\hline LEU07-OP1-Z014 & 20.9 & 4.7 & 950 & 1 & flowing $\mathrm{He}$ & 20 & 1800 & 1 & vacuum \\
\hline LEU07-OP1-Z015 & 21.7 & 4.7 & 950 & 1 & flowing $\mathrm{He}$ & 20 & 1800 & 1 & vacuum \\
\hline LEU07-OP1-Z016 & 20.9 & 4.7 & 950 & 1 & flowing $\mathrm{He}$ & 20 & 1800 & 1 & vacuum \\
\hline LEU07-OP1-Z017 & 20.9 & 4.7 & 950 & 1 & flowing $\mathrm{He}$ & 20 & 1800 & 1 & vacuum \\
\hline LEU07-OP1-Z018 & 20.9 & 4.7 & 950 & 1 & flowing $\mathrm{He}$ & 20 & 1800 & 1 & vacuum \\
\hline LEU07-OP1-Z019 & 20.9 & 4.7 & 950 & 1 & flowing $\mathrm{He}$ & 20 & 1800 & 1 & vacuum \\
\hline LEU07-OP1-Z020 & 20.9 & 4.7 & 950 & 1 & flowing $\mathrm{He}$ & 20 & 1800 & 1 & vacuum \\
\hline LEU07-OP1-Z021 & 20.9 & 4.7 & 950 & 1 & flowing $\mathrm{He}$ & 20 & 1800 & 1 & vacuum \\
\hline LEU07-OP1-Z022 & 20.9 & 4.7 & 950 & 1 & flowing $\mathrm{He}$ & 20 & 1800 & 1 & vacuum \\
\hline LEU07-OP1-Z023 & 20.9 & 4.7 & 950 & 1 & flowing $\mathrm{He}$ & 20 & 1800 & 1 & vacuum \\
\hline LEU07-OP1-Z024 & 20.9 & 4.7 & 950 & 1 & flowing $\mathrm{He}$ & 20 & 1800 & 1 & vacuum \\
\hline LEU07-OP1-Z025 & 20.9 & 4.7 & 950 & 1 & flowing $\mathrm{He}$ & 20 & 1800 & 1 & vacuum \\
\hline LEU07-OP1-Z026 & 20.9 & 4.7 & 950 & 1 & flowing $\mathrm{He}$ & 20 & 1800 & 1 & vacuum \\
\hline LEU07-OP1-Z027 & 20.9 & 4.7 & 950 & 1 & flowing $\mathrm{He}$ & 20 & 1800 & 1 & vacuum \\
\hline LEU07-OP1-Z028 & 20.9 & 4.7 & 950 & 1 & flowing $\mathrm{He}$ & 20 & 1800 & 1 & vacuum \\
\hline LEU07-OP1-Z029 & 20.9 & 4.7 & 950 & 1 & flowing $\mathrm{He}$ & 20 & 1800 & 1 & vacuum \\
\hline LEU07-OP1-Z030 & 20.9 & 4.7 & 950 & 1 & flowing $\mathrm{He}$ & 20 & 1800 & 1 & vacuum \\
\hline
\end{tabular}

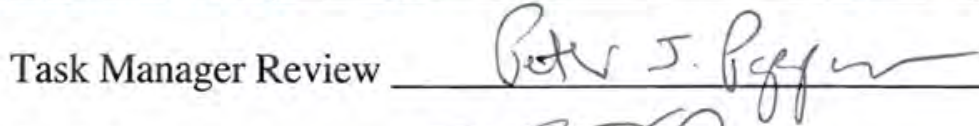

Date $12-23-09$

QAS Review

Date $12 / 23 / 0 \%$ 
Table 3-2 (cont.): Summary of process conditions used in making LEU07-OP1-Z (AGR-2 UCO Baseline) compacts

\begin{tabular}{|c|c|c|c|c|c|}
\hline \multirow{2}{*}{ Compact ID } & \multicolumn{5}{|c|}{ Carbonization Parameter } \\
\hline & $\begin{array}{l}\text { Molding Pressure } \\
(\mathrm{MPa})\end{array}$ & $\begin{array}{l}\text { Heating Rate } \\
\left({ }^{\circ} \mathrm{C} / \mathrm{min} .\right)\end{array}$ & $\begin{array}{l}\text { Max. Temp. } \\
\left({ }^{\circ} \mathrm{C}\right)\end{array}$ & $\begin{array}{l}\text { Hold Time } \\
\text { (hrs.) }\end{array}$ & Atmosphere \\
\hline LEU07-OP1-Z031 & 20.9 & 4.7 & 950 & 1 & flowing $\mathrm{He}$ \\
\hline LEU07-OP1-Z032 & 20.9 & 4.7 & 950 & 1 & flowing $\mathrm{He}$ \\
\hline LEU07-OP1-Z033 & 21.7 & 4.7 & 950 & 1 & flowing $\mathrm{He}$ \\
\hline LEU07-OP1-Z034 & 20.9 & 4.7 & 950 & 1 & flowing $\mathrm{He}$ \\
\hline LEU07-OP1-Z035 & 22.4 & 4.7 & 950 & 1 & flowing $\mathrm{He}$ \\
\hline LEU07-OP1-Z036 & 20.9 & 4.7 & 950 & 1 & flowing $\mathrm{He}$ \\
\hline LEU07-OP1-Z037 & 20.9 & 4.7 & 950 & 1 & flowing $\mathrm{He}$ \\
\hline LEU07-OP1-Z038 & 20.9 & 4.7 & 950 & 1 & flowing $\mathrm{He}$ \\
\hline LEU07-OP1-Z039 & 20.9 & 4.7 & 950 & 1 & flowing $\mathrm{He}$ \\
\hline LEU07-OP1-Z040 & 20.9 & 4.7 & 950 & 1 & flowing $\mathrm{He}$ \\
\hline LEU07-OP1-Z041 & 20.9 & 4.7 & 950 & 1 & flowing $\mathrm{He}$ \\
\hline LEU07-OP1-Z042 & 20.9 & 4.7 & 950 & 1 & flowing $\mathrm{He}$ \\
\hline LEU07-OP1-Z043 & 20.9 & 4.7 & 950 & 1 & flowing $\mathrm{He}$ \\
\hline LEU07-OP1-Z044 & 21.7 & 4.7 & 950 & 1 & flowing $\mathrm{He}$ \\
\hline LEU07-OP1-Z045 & 20.9 & 4.7 & 950 & 1 & flowing $\mathrm{He}$ \\
\hline LEU07-OP1-Z046 & 20.9 & 4.7 & 950 & 1 & flowing $\mathrm{He}$ \\
\hline LEU07-OP1-Z047 & 20.9 & 4.7 & 950 & 1 & flowing $\mathrm{He}$ \\
\hline LEU07-OP1-Z048 & 20.9 & 4.7 & 950 & 1 & flowing $\mathrm{He}$ \\
\hline LEU07-OP1-Z049 & 21.7 & 4.7 & 950 & 1 & flowing $\mathrm{He}$ \\
\hline LEU07-OP1-Z050 & 20.9 & 4.7 & 950 & 1 & flowing $\mathrm{He}$ \\
\hline LEU07-OP1-Z051 & 22.4 & 4.7 & 950 & 1 & flowing $\mathrm{He}$ \\
\hline LEU07-OP1-Z052 & 20.9 & 4.7 & 950 & 1 & flowing $\mathrm{He}$ \\
\hline LEU07-OP1-Z053 & 20.9 & 4.7 & 950 & 1 & flowing $\mathrm{He}$ \\
\hline LEU07-OP1-Z054 & 20.9 & 4.7 & 950 & 1 & flowing $\mathrm{He}$ \\
\hline LEU07-OP1-Z055 & 20.9 & 4.7 & 950 & 1 & flowing $\mathrm{He}$ \\
\hline LEU07-OP1-Z056 & 20.9 & 4.7 & 950 & 1 & flowing $\mathrm{He}$ \\
\hline LEU07-OP1-Z057 & 20.9 & 4.7 & 950 & 1 & flowing $\mathrm{He}$ \\
\hline LEU07-OP1-Z058 & 20.9 & 4.7 & 950 & 1 & flowing $\mathrm{He}$ \\
\hline LEU07-OPI-Z059 & 21.7 & 4.7 & 950 & 1 & flowing $\mathrm{He}$ \\
\hline LEU07-OP1-Z060 & 20.9 & 4.7 & 950 & 1 & flowing $\mathrm{He}$ \\
\hline
\end{tabular}

\begin{tabular}{|c|c|c|c|}
\hline \multicolumn{4}{|c|}{ Heat-treatment Parameters } \\
\hline $\begin{array}{l}\text { Heating Rate } \\
\left({ }^{\circ} \mathrm{C} / \mathrm{min} .\right)\end{array}$ & $\begin{array}{l}\text { Max. Temp. } \\
\left({ }^{\circ} \mathrm{C}\right)\end{array}$ & $\begin{array}{l}\text { Hold Time } \\
\text { (hrs.) }\end{array}$ & Atmosphere \\
\hline 20 & 1800 & 1 & vacuum \\
\hline 20 & 1800 & 1 & vacuum \\
\hline 20 & 1800 & 1 & vacuum \\
\hline 20 & 1800 & 1 & vacuum \\
\hline 20 & 1800 & 1 & vacuum \\
\hline 20 & 1800 & 1 & vacuum \\
\hline 20 & 1800 & 1 & vacuum \\
\hline 20 & 1800 & 1 & vacuum \\
\hline 20 & 1800 & 1 & vacuum \\
\hline 20 & 1800 & 1 & vacuum \\
\hline 20 & 1800 & 1 & vacuum \\
\hline 20 & 1800 & 1 & vacuum \\
\hline 20 & 1800 & 1 & vacuum \\
\hline 20 & 1800 & 1 & vacuum \\
\hline 20 & 1800 & 1 & vacuum \\
\hline 20 & 1800 & 1 & vacuum \\
\hline 20 & 1800 & 1 & vacuum \\
\hline 20 & 1800 & 1 & vacuum \\
\hline 20 & 1800 & 1 & vacuum \\
\hline 20 & 1800 & 1 & vacuum \\
\hline 20 & 1800 & 1 & vacuum \\
\hline 20 & 1800 & 1 & vacuum \\
\hline 20 & 1800 & 1 & vacuum \\
\hline 20 & 1800 & 1 & vacuum \\
\hline 20 & 1800 & 1 & vacuum \\
\hline 20 & 1800 & 1 & vacuum \\
\hline 20 & 1800 & 1 & vacuum \\
\hline 20 & 1800 & 1 & vacuum \\
\hline 20 & 1800 & 1 & vacuum \\
\hline 20 & 1800 & 1 & vacuum \\
\hline
\end{tabular}

Date $12-23-09$

Date $12 / 23 / 09$ 
Table 3-2 (cont.): Summary of process conditions used in making LEU07-OP1-Z (AGR-2 UCO Baseline) compacts

\begin{tabular}{|c|c|c|c|c|c|c|c|c|c|}
\hline \multirow{2}{*}{ Compact ID } & \multicolumn{5}{|c|}{ Carbonization Parameter } & \multicolumn{4}{|c|}{ Heat-treatment Parameters } \\
\hline & $\begin{array}{l}\text { Molding Pressure } \\
(\mathrm{MPa})\end{array}$ & $\begin{array}{l}\text { Heating Rate } \\
\left({ }^{\circ} \mathrm{C} / \mathrm{min} .\right)\end{array}$ & $\begin{array}{l}\text { Max. Temp. } \\
\left({ }^{\circ} \mathrm{C}\right)\end{array}$ & $\begin{array}{l}\text { Hold Time } \\
\text { (hrs.) }\end{array}$ & Atmosphere & $\begin{array}{l}\text { Heating Rate } \\
\left({ }^{\circ} \mathrm{C} / \mathrm{min} .\right)\end{array}$ & $\begin{array}{l}\text { Max. Temp. } \\
\left({ }^{\circ} \mathrm{C}\right)\end{array}$ & $\begin{array}{l}\text { Hold Time } \\
\text { (hrs.) }\end{array}$ & Atmosphere \\
\hline LEU07-OP1-Z061 & 20.9 & 4.7 & 950 & 1 & flowing $\mathrm{He}$ & 20 & 1800 & 1 & vacuum \\
\hline LEU07-OP1-Z062 & 20.9 & 4.7 & 950 & 1 & flowing $\mathrm{He}$ & 20 & 1800 & 1 & vacuum \\
\hline LEU07-OP1-Z063 & 20.9 & 4.7 & 950 & 1 & flowing $\mathrm{He}$ & 20 & 1800 & 1 & vacuum \\
\hline LEU07-OP1-Z064 & 20.9 & 4.7 & 950 & 1 & flowing $\mathrm{He}$ & 20 & 1800 & 1 & vacuum \\
\hline LEU07-OP1-Z065 & 22.4 & 4.7 & 950 & 1 & flowing $\mathrm{He}$ & 20 & 1800 & 1 & vacuum \\
\hline LEU07-OP1-Z066 & 20.9 & 4.7 & 950 & 1 & flowing $\mathrm{He}$ & 20 & 1800 & 1 & vacuum \\
\hline LEU07-OP1-Z067 & 20.9 & 4.7 & 950 & 1 & flowing $\mathrm{He}$ & 20 & 1800 & 1 & vacuum \\
\hline LEU07-OP1-Z068 & 20.9 & 4.7 & 950 & 1 & flowing $\mathrm{He}$ & 20 & 1800 & 1 & vacuum \\
\hline LEU07-OP1-Z069 & 20.9 & 4.7 & 950 & 1 & flowing $\mathrm{He}$ & 20 & 1800 & 1 & vacuum \\
\hline LEU07-OP1-Z070 & 20.9 & 4.7 & 950 & 1 & flowing $\mathrm{He}$ & 20 & 1800 & 1 & vacuum \\
\hline LEU07-OP1-Z071 & 20.9 & 4.7 & 950 & 1 & flowing $\mathrm{He}$ & 20 & 1800 & 1 & vacuum \\
\hline LEU07-OP1-Z072 & 20.9 & 4.7 & 950 & 1 & flowing $\mathrm{He}$ & 20 & 1800 & 1 & vacuum \\
\hline LEU07-OP1-Z073 & 20.9 & 4.7 & 950 & 1 & flowing $\mathrm{He}$ & 20 & 1800 & 1 & vacuum \\
\hline LEU07-OP1-Z074 & 20.9 & 4.7 & 950 & 1 & flowing $\mathrm{He}$ & 20 & 1800 & 1 & vacuum \\
\hline LEU07-OP1-Z075 & 20.9 & 4.7 & 950 & 1 & flowing $\mathrm{He}$ & 20 & 1800 & 1 & vacuum \\
\hline LEU07-OP1-Z076 & 20.9 & 4.7 & 950 & 1 & flowing $\mathrm{He}$ & 20 & 1800 & 1 & vacuum \\
\hline LEU07-OP1-Z077 & 22.4 & 4.7 & 950 & 1 & flowing $\mathrm{He}$ & 20 & 1800 & 1 & vacuum \\
\hline LEU07-OP1-Z078 & 20.9 & 4.7 & 950 & 1 & flowing $\mathrm{He}$ & 20 & 1800 & 1 & vacuum \\
\hline LEU07-OP1-Z079 & 20.9 & 4.7 & 950 & 1 & flowing $\mathrm{He}$ & 20 & 1800 & 1 & vacuum \\
\hline LEU07-OP1-Z080 & 20.9 & 4.7 & 950 & 1 & flowing $\mathrm{He}$ & 20 & 1800 & 1 & vacuum \\
\hline LEU07-OP1-Z081 & 20.9 & 4.7 & 950 & 1 & flowing $\mathrm{He}$ & 20 & 1800 & 1 & vacuum \\
\hline LEU07-OP1-Z082 & 20.9 & 4.7 & 950 & 1 & flowing $\mathrm{He}$ & 20 & 1800 & 1 & vacuum \\
\hline LEU07-OP1-Z083 & 20.9 & 4.7 & 950 & 1 & flowing $\mathrm{He}$ & 20 & 1800 & 1 & vacuum \\
\hline LEU07-OP1-Z084 & 20.9 & 4.7 & 950 & 1 & flowing $\mathrm{He}$ & 20 & 1800 & 1 & vacuum \\
\hline LEU07-OP1-Z085 & 20.9 & 4.7 & 950 & 1 & flowing $\mathrm{He}$ & 20 & 1800 & 1 & vacuum \\
\hline LEU07-OP1-Z086 & 20.9 & 4.7 & 950 & 1 & flowing $\mathrm{He}$ & 20 & 1800 & 1 & vacuum \\
\hline LEU07-OP1-Z087 & 20.9 & 4.7 & 950 & 1 & flowing $\mathrm{He}$ & 20 & 1800 & 1 & vacuum \\
\hline LEU07-OP1-Z088 & 20.9 & 4.7 & 950 & 1 & flowing $\mathrm{He}$ & 20 & 1800 & 1 & vacuum \\
\hline LEU07-OP1-Z089 & 20.9 & 4.7 & 950 & 1 & flowing $\mathrm{He}$ & 20 & 1800 & 1 & vacuum \\
\hline LEU07-OP1-Z090 & 20.9 & 4.7 & 950 & 1 & flowing $\mathrm{He}$ & 20 & 1800 & 1 & vacuum \\
\hline
\end{tabular}


Table 3-2 (cont.): Summary of process conditions used in making LEU07-OP1-Z (AGR-2 UCO Baseline) compacts

\begin{tabular}{|c|c|c|c|c|c|c|c|c|c|}
\hline \multirow{2}{*}{ Compact ID } & \multicolumn{5}{|c|}{ Carbonization Parameter } & \multicolumn{4}{|c|}{ Heat-treatment Parameters } \\
\hline & $\begin{array}{l}\text { Molding Pressure } \\
(\mathrm{MPa})\end{array}$ & $\begin{array}{l}\text { Heating Rate } \\
\left({ }^{\circ} \mathrm{C} / \mathrm{min} .\right)\end{array}$ & $\begin{array}{l}\text { Max. Temp. } \\
\left({ }^{\circ} \mathrm{C}\right)\end{array}$ & $\begin{array}{l}\text { Hold Time } \\
\text { (hrs.) }\end{array}$ & Atmosphere & $\begin{array}{l}\text { Heating Rate } \\
\left({ }^{\circ} \mathrm{C} / \mathrm{min} .\right)\end{array}$ & $\begin{array}{l}\text { Max. Temp. } \\
\left({ }^{\circ} \mathrm{C}\right)\end{array}$ & $\begin{array}{l}\text { Hold Time } \\
\text { (hrs.) }\end{array}$ & Atmosphere \\
\hline LEU07-OP1-Z091 & 20.9 & 4.7 & 950 & 1 & flowing $\mathrm{He}$ & 20 & 1800 & 1 & vacuum \\
\hline LEU07-OP1-Z092 & 20.9 & 4.7 & 950 & 1 & flowing $\mathrm{He}$ & 20 & 1800 & 1 & vacuum \\
\hline LEU07-OP1-Z093 & 20.9 & 4.7 & 950 & 1 & flowing $\mathrm{He}$ & 20 & 1800 & 1 & vacuum \\
\hline LEU07-OP1-Z094 & 20.9 & 4.7 & 950 & 1 & flowing $\mathrm{He}$ & 20 & 1800 & 1 & vacuum \\
\hline LEU07-OP1-Z095 & 20.9 & 4.7 & 950 & 1 & flowing $\mathrm{He}$ & 20 & 1800 & 1 & vacuum \\
\hline LEU07-OP1-Z096 & 20.9 & 4.7 & 950 & 1 & flowing $\mathrm{He}$ & 20 & 1800 & 1 & vacuum \\
\hline LEU07-OP1-Z097 & 20.9 & 4.7 & 950 & 1 & flowing $\mathrm{He}$ & 20 & 1800 & 1 & vacuum \\
\hline LEU07-OP1-Z098 & 20.9 & 4.7 & 950 & 1 & flowing $\mathrm{He}$ & 20 & 1800 & 1 & vacuum \\
\hline LEU07-OP1-Z099 & 20.9 & 4.7 & 950 & 1 & flowing $\mathrm{He}$ & 20 & 1800 & 1 & vacuum \\
\hline LEU07-OP1-Z100 & 20.9 & 4.7 & 950 & 1 & flowing $\mathrm{He}$ & 20 & 1800 & 1 & vacuum \\
\hline LEU07-OP1-Z101 & 20.9 & 4.7 & 950 & 1 & flowing $\mathrm{He}$ & 20 & 1800 & 1 & vacuum \\
\hline LEU07-OP1-Z102 & 20.9 & 4.7 & 950 & 1 & flowing $\mathrm{He}$ & 20 & 1800 & 1 & vacuum \\
\hline LEU07-OP1-Z103 & 20.9 & 4.7 & 950 & 1 & flowing $\mathrm{He}$ & 20 & 1800 & 1 & vacuum \\
\hline LEU07-OP1-Z104 & 20.9 & 4.7 & 950 & 1 & flowing $\mathrm{He}$ & 20 & 1800 & 1 & vacuum \\
\hline LEU07-OP1-Z105 & 20.9 & 4.7 & 950 & 1 & flowing $\mathrm{He}$ & 20 & 1800 & 1 & vacuum \\
\hline LEU07-OP1-Z106 & 20.9 & 4.7 & 950 & 1 & flowing $\mathrm{He}$ & 20 & 1800 & 1 & vacuum \\
\hline LEU07-OP1-Z107 & 20.9 & 4.7 & 950 & 1 & flowing $\mathrm{He}$ & 20 & 1800 & 1 & vacuum \\
\hline LEU07-OP1-Z108 & 20.9 & 4.7 & 950 & 1 & flowing $\mathrm{He}$ & 20 & 1800 & 1 & vacuum \\
\hline LEU07-OP1-Z109 & 22.4 & 4.7 & 950 & 1 & flowing $\mathrm{He}$ & 20 & 1800 & 1 & vacuum \\
\hline LEU07-OP1-Z110 & 20.9 & 4.7 & 950 & 1 & flowing $\mathrm{He}$ & 20 & 1800 & 1 & vacuum \\
\hline LEU07-OP1-Z111 & 22.1 & 4.7 & 950 & 1 & flowing $\mathrm{He}$ & 20 & 1800 & 1 & vacuum \\
\hline LEU07-OP1-Z112 & 21.7 & 4.7 & 950 & 1 & flowing $\mathrm{He}$ & 20 & 1800 & 1 & vacuum \\
\hline LEU07-OP1-Z113 & 20.9 & 4.7 & 950 & 1 & flowing $\mathrm{He}$ & 20 & 1800 & 1 & vacuum \\
\hline LEU07-OP1-Z114 & 20.9 & 4.7 & 950 & 1 & flowing $\mathrm{He}$ & 20 & 1800 & 1 & vacuum \\
\hline LEU07-OP1-Z115 & 20.9 & 4.7 & 950 & 1 & flowing $\mathrm{He}$ & 20 & 1800 & 1 & vacuum \\
\hline LEU07-OP1-Z116 & 20.9 & 4.7 & 950 & 1 & flowing $\mathrm{He}$ & 20 & 1800 & 1 & vacuum \\
\hline LEU07-OP1-Z117 & 20.9 & 4.7 & 950 & 1 & flowing $\mathrm{He}$ & 20 & 1800 & 1 & vacuum \\
\hline LEU07-OP1-Z118 & 20.9 & 4.7 & 950 & 1 & flowing $\mathrm{He}$ & 20 & 1800 & 1 & vacuum \\
\hline LEU07-OP1-Z119 & 20.9 & 4.7 & 950 & 1 & flowing $\mathrm{He}$ & 20 & 1800 & 1 & vacuum \\
\hline LEU07-OP1-Z120 & 20.9 & 4.7 & 950 & 1 & flowing $\mathrm{He}$ & 20 & 1800 & 1 & vacuum \\
\hline
\end{tabular}

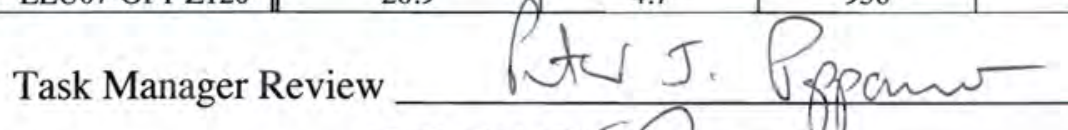

Date $12-23-09$

QAS Review

Date $12 / 23 / 6 \%$ 
Table 3-2 (cont.): Summary of process conditions used in making LEU07-OP1-Z (AGR-2 UCO Baseline) compacts

\begin{tabular}{|c|c|c|c|c|c|}
\hline \multirow{2}{*}{ Compact ID } & \multicolumn{5}{|c|}{ Carbonization Parameter } \\
\hline & $\begin{array}{l}\text { Molding Pressure } \\
(\mathrm{MPa})\end{array}$ & $\begin{array}{l}\text { Heating Rate } \\
\left({ }^{\circ} \mathrm{C} / \mathrm{min} .\right)\end{array}$ & $\begin{array}{l}\text { Max. Temp. } \\
\left({ }^{\circ} \mathrm{C}\right)\end{array}$ & $\begin{array}{l}\text { Hold Time } \\
\text { (hrs.) }\end{array}$ & Atmosphere \\
\hline LEU07-OP1-Z121 & 20.9 & 4.7 & 950 & 1 & flowing $\mathrm{He}$ \\
\hline LEU07-OP1-Z122 & 22.4 & 4.7 & 950 & 1 & flowing $\mathrm{He}$ \\
\hline LEU07-OP1-Z123 & 22.4 & 4.7 & 950 & 1 & flowing $\mathrm{He}$ \\
\hline LEU07-OP1-Z124 & 20.9 & 4.7 & 950 & 1 & flowing $\mathrm{He}$ \\
\hline LEU07-OP1-Z125 & 20.9 & 4.7 & 950 & 1 & flowing $\mathrm{He}$ \\
\hline LEU07-OP1-Z126 & 20.9 & 4.7 & 950 & 1 & flowing $\mathrm{He}$ \\
\hline LEU07-OP1-Z127 & 20.9 & 4.7 & 950 & 1 & flowing $\mathrm{He}$ \\
\hline LEU07-OP1-Z128 & 20.9 & 4.7 & 950 & 1 & flowing $\mathrm{He}$ \\
\hline LEU07-OP1-Z129 & 20.9 & 4.7 & 950 & 1 & flowing $\mathrm{He}$ \\
\hline LEU07-OP1-Z130 & 21.7 & 4.7 & 950 & 1 & flowing $\mathrm{He}$ \\
\hline LEU07-OP1-Z131 & 20.9 & 4.7 & 950 & 1 & flowing $\mathrm{He}$ \\
\hline LEU07-OP1-Z132 & 20.9 & 4.7 & 950 & 1 & flowing $\mathrm{He}$ \\
\hline LEU07-OP1-Z133 & 20.9 & 4.7 & 950 & 1 & flowing $\mathrm{He}$ \\
\hline LEU07-OP1-Z134 & 20.9 & 4.7 & 950 & 1 & flowing $\mathrm{He}$ \\
\hline LEU07-OP1-Z135 & 20.9 & 4.7 & 950 & 1 & flowing $\mathrm{He}$ \\
\hline LEU07-OP1-Z136 & 20.9 & 4.7 & 950 & 1 & flowing $\mathrm{He}$ \\
\hline LEU07-OP1-Z137 & 20.9 & 4.7 & 950 & 1 & flowing $\mathrm{He}$ \\
\hline LEU07-OP1-Z138 & 20.9 & 4.7 & 950 & 1 & flowing $\mathrm{He}$ \\
\hline LEU07-OP1-Z139 & 20.9 & 4.7 & 950 & 1 & flowing $\mathrm{He}$ \\
\hline LEU07-OP1-Z140 & 20.9 & 4.7 & 950 & 1 & flowing $\mathrm{He}$ \\
\hline LEU07-OP1-Z141 & 20.9 & 4.7 & 950 & 1 & flowing $\mathrm{He}$ \\
\hline LEU07-OP1-Z142 & 20.9 & 4.7 & 950 & 1 & flowing $\mathrm{He}$ \\
\hline LEU07-OP1-Z143 & 20.9 & 4.7 & 950 & 1 & flowing $\mathrm{He}$ \\
\hline LEU07-OP1-Z144 & 20.9 & 4.7 & 950 & 1 & flowing $\mathrm{He}$ \\
\hline LEU07-OP1-Z145 & 20.9 & 4.7 & 950 & 1 & flowing $\mathrm{He}$ \\
\hline LEU07-OP1-Z146 & 20.9 & 4.7 & 950 & 1 & flowing $\mathrm{He}$ \\
\hline LEU07-OP1-Z147 & 20.9 & 4.7 & 950 & 1 & flowing $\mathrm{He}$ \\
\hline LEU07-OP1-Z148 & 20.9 & 4.7 & 950 & 1 & flowing $\mathrm{He}$ \\
\hline LEU07-OP1-Z149 & 20.9 & 4.7 & 950 & 1 & flowing $\mathrm{He}$ \\
\hline LEU07-OP1-Z150 & 20.9 & 4.7 & 950 & 1 & flowing $\mathrm{He}$ \\
\hline
\end{tabular}

\begin{tabular}{|c|c|c|c|}
\hline \multicolumn{4}{|c|}{ Heat-treatment Parameters } \\
\hline $\begin{array}{l}\text { Heating Rate } \\
\left({ }^{\circ} \mathrm{C} / \mathrm{min} .\right)\end{array}$ & $\begin{array}{c}\text { Max. Temp. } \\
\left({ }^{\circ} \mathrm{C}\right)\end{array}$ & $\begin{array}{l}\text { Hold Time } \\
\text { (hrs.) }\end{array}$ & Atmosphere \\
\hline 20 & 1800 & 1 & vacuum \\
\hline 20 & 1800 & 1 & vacuum \\
\hline 20 & 1800 & 1 & vacuum \\
\hline 20 & 1800 & 1 & vacuum \\
\hline 20 & 1800 & 1 & vacuum \\
\hline 20 & 1800 & 1 & vacuum \\
\hline 20 & 1800 & 1 & vacuum \\
\hline 20 & 1800 & 1 & vacuum \\
\hline 20 & 1800 & 1 & vacuum \\
\hline 20 & 1800 & 1 & vacuum \\
\hline 20 & 1800 & 1 & vacuum \\
\hline 20 & 1800 & 1 & vacuum \\
\hline 20 & 1800 & 1 & vacuum \\
\hline 20 & 1800 & 1 & vacuum \\
\hline 20 & 1800 & 1 & vacuum \\
\hline 20 & 1800 & 1 & vacuum \\
\hline 20 & 1800 & 1 & vacuum \\
\hline 20 & 1800 & 1 & vacuum \\
\hline 20 & 1800 & 1 & vacuum \\
\hline 20 & 1800 & 1 & vacuum \\
\hline 20 & 1800 & 1 & vacuum \\
\hline 20 & 1800 & 1 & vacuum \\
\hline 20 & 1800 & 1 & vacuum \\
\hline 20 & 1800 & 1 & vacuum \\
\hline 20 & 1800 & 1 & vacuum \\
\hline 20 & 1800 & 1 & vacuum \\
\hline 20 & 1800 & 1 & vacuum \\
\hline 20 & 1800 & 1 & vacuum \\
\hline 20 & 1800 & 1 & vacuum \\
\hline 20 & 1800 & 1 & vacuum \\
\hline
\end{tabular}

Date $12-23-09$

Date $\angle 2 / 23 / 09$ 
Table 3-2 (cont.): Summary of process conditions used in making LEU07-OP1-Z (AGR-2 UCO Variant) compacts

\begin{tabular}{|c|c|c|c|c|c|}
\hline \multirow{2}{*}{ Compact ID } & \multicolumn{5}{|c|}{ Carbonization Parameter } \\
\hline & $\begin{array}{l}\text { Molding Pressure } \\
(\mathrm{MPa})\end{array}$ & $\begin{array}{c}\text { Heating Rate } \\
\left({ }^{\circ} \mathrm{C} / \mathrm{min} .\right)\end{array}$ & $\begin{array}{l}\text { Max. Temp. } \\
\left({ }^{\circ} \mathrm{C}\right)\end{array}$ & $\begin{array}{l}\text { Hold Time } \\
\text { (hrs.) }\end{array}$ & Atmosphere \\
\hline LEU07-OP1-Z151 & 20.9 & 4.7 & 950 & 1 & flowing $\mathrm{He}$ \\
\hline LEU07-OP1-Z152 & 20.9 & 4.7 & 950 & 1 & flowing $\mathrm{He}$ \\
\hline LEU07-OP1-Z153 & 20.9 & 4.7 & 950 & 1 & flowing $\mathrm{He}$ \\
\hline LEU07-OP1-Z154 & 20.9 & 4.7 & 950 & 1 & flowing $\mathrm{He}$ \\
\hline LEU07-OP1-Z155 & 20.9 & 4.7 & 950 & 1 & flowing $\mathrm{He}$ \\
\hline & & & & & \\
\hline & & & & & \\
\hline & & & & & \\
\hline & & & & & \\
\hline & & & & & \\
\hline & & & & & \\
\hline & & & & & \\
\hline & & & & & \\
\hline & & & & & \\
\hline & & & & & \\
\hline & & & & & \\
\hline & & & & & \\
\hline & & & & & \\
\hline & & & & & \\
\hline & & & & & \\
\hline & & & & & \\
\hline & & & & & \\
\hline & & & & & \\
\hline & & & & & \\
\hline & & & & & \\
\hline & & & & & \\
\hline & & & & & \\
\hline & & & & & \\
\hline & & & & & \\
\hline
\end{tabular}

Task Manager Review feter if. Geppano

QAS Review

\begin{tabular}{|c|c|c|c|}
\hline \multicolumn{4}{|c|}{ Heat-treatment Parameters } \\
\hline $\begin{array}{l}\text { Heating Rate } \\
\left({ }^{\circ} \mathrm{C} / \mathrm{min} .\right)\end{array}$ & $\begin{array}{l}\text { Max. Temp. } \\
\left({ }^{\circ} \mathrm{C}\right)\end{array}$ & $\begin{array}{l}\text { Hold Time } \\
\text { (hrs.) }\end{array}$ & Atmosphere \\
\hline 20 & 1800 & 1 & vacuum \\
\hline 20 & 1800 & 1 & vacuum \\
\hline 20 & 1800 & 1 & vacuum \\
\hline 20 & 1800 & 1 & vacuum \\
\hline 20 & 1800 & 1 & vacuum \\
\hline & & & \\
\hline & & & \\
\hline & & & \\
\hline & & & \\
\hline & & & \\
\hline & & & \\
\hline & & & \\
\hline & & & \\
\hline & & & \\
\hline & & & \\
\hline & & & \\
\hline & & & \\
\hline & & & \\
\hline & & & \\
\hline & & & \\
\hline & & & \\
\hline & & & \\
\hline & & & \\
\hline & & & \\
\hline & & & \\
\hline & & & \\
\hline & & & \\
\hline
\end{tabular}

Date $12-23-09$

Date $12 / 23 / 09$ 


\section{$4 \quad$ Impurity analysis of matrix, resin, and graphites}

The AGR-2 Fuel Specification (SPC-923) puts maximum limits on the elemental impurities Al, $\mathrm{Ca}, \mathrm{Ti}, \mathrm{V}, \mathrm{Cr}, \mathrm{Mn}, \mathrm{Fe}, \mathrm{Co}$, and $\mathrm{Ni}$. The natural graphite, synthetic graphite, and thermosetting resin used to make the matrix/overcoat material may contain these impurities. Therefore, the selection of graphites and resin used to make the matrix must have low concentrations of these impurities to ensure that the compacts made from the matrix will be within specification. Subsequently, part of the compacting development effort was selection and qualification of natural graphite, synthetic graphite, and resin. A graphite or resin was considered "qualified" if it could produce a compact that was within specification on impurities. The AGR-1 compacts showed that compacts could be made from these matrix constituents and pass the impurity specification. The qualification process involved receiving natural graphite and synthetic graphite and testing them via glow discharge mass spectrometry (GDMS) in order to establish their initial impurity concentrations. The graphites and resin were then combined to produce matrix that was carbonized and heat treated in powder form. The impurity levels in the heat treated matrix was then also measured by GDMS.

Table 4-1 shows the initial impurity levels for the natural graphite and synthetic graphite that were used to make LEU07-OP1-Z compacts. Natural graphite (Asbury Graphite Mills RD13371), synthetic graphite (SGL Carbon KRB2000), and thermosetting resin (Hexion Durite SC1008-lot LK8JD0450) were combined in a weight ratio of 64:16:20 to make the matrix. Four batches of matrix were produced: RDKrS-011309, RDKrS-012909, RDKrS-020209, and RDKrS-021309. A sample of the RDKrS-011309 matrix was carbonized and heat treated in powder form prior to being tested for impurities by GDMS. The other matrix batches were not tested because they were made up of the same starting materials. Notice that the heat treatment processes significantly reduced impurity levels in the matrix for several elements.

Table 4-1: Matrix constituents that were used in AGR-2 LEU07-OP1-Z compacts

\begin{tabular}{|c|c|c|c|}
\hline \multirow[b]{2}{*}{ Element } & \multicolumn{3}{|c|}{ Impurity concentration (ppm) } \\
\hline & $\begin{array}{c}\text { Natural Graphite- } \\
\text { RD13371 }\end{array}$ & $\begin{array}{c}\text { Synthetic Graphite- } \\
\text { KRB2000 }\end{array}$ & $\begin{array}{l}\text { Heat treated Matrix- } \\
\text { RDKrS-011309 }\end{array}$ \\
\hline Element & Concentration (ppm) & Concentration (ppm) & Concentration (ppm) \\
\hline $\mathrm{Al}$ & 36 & 0.35 & $<0.05$ \\
\hline $\mathrm{Ca}$ & 9.4 & 0.7 & 0.45 \\
\hline $\mathrm{Ti}$ & 0.43 & 0.06 & 0.73 \\
\hline $\mathrm{V}$ & 0.6 & 0.02 & 6.3 \\
\hline $\mathrm{Cr}$ & 4.5 & $<0.5$ & $<0.5$ \\
\hline $\mathrm{Mn}$ & 0.54 & $<0.05$ & $<0.05$ \\
\hline $\mathrm{Fe}$ & 34 & 1.4 & 0.03 \\
\hline Co & $<0.05$ & 0.25 & $<0.05$ \\
\hline $\mathrm{Ni}$ & 0.37 & 1.2 & $<0.1$ \\
\hline
\end{tabular}

The following pages show the impurity analysis reports for the natural graphite, synthetic graphite, and matrix sample listed in Table 4-1. Also attached is the certificate of analysis for the resin from Hexion. Note that an expiration date was set for the resin of 6 months from the manufacture date. LEU07-OP1-Z compacting was completed on 4/02/09. 


\section{Customer: UT-Battelle Oak Ridge \\ Date: \\ 27-Dec-03}

Customer ID: Graphite

AGM RD13371

Element
Concentration

[ppm wt ]

\begin{tabular}{|c|c|}
\hline $\mathrm{Li}$ & $<0.01$ \\
\hline $\mathrm{Be}$ & $<0.01$ \\
\hline $\mathrm{B}$ & 0.17 \\
\hline $\mathrm{C}$ & Matrix \\
\hline $\mathrm{N}$ & - \\
\hline $\mathrm{O}$ & - \\
\hline $\mathrm{F}$ & $<5$ \\
\hline $\mathrm{Na}$ & 2.9 \\
\hline $\mathrm{Mg}$ & 4.8 \\
\hline $\mathrm{Al}$ & 36 \\
\hline $\mathrm{Si}$ & 240 \\
\hline $\mathrm{P}$ & 1.6 \\
\hline $\mathrm{S}$ & 85 \\
\hline $\mathrm{Cl}$ & 0.8 \\
\hline $\mathrm{K}$ & 1.5 \\
\hline $\mathrm{Ca}$ & 9.4 \\
\hline $\mathrm{Sc}$ & $<0.05$ \\
\hline $\mathrm{Ti}$ & 0.43 \\
\hline $\mathrm{V}$ & 0.6 \\
\hline $\mathrm{Cr}$ & 4.5 \\
\hline $\mathrm{Mn}$ & 0.54 \\
\hline $\mathrm{Fe}$ & 34 \\
\hline $\mathrm{Co}$ & $<0.05$ \\
\hline $\mathrm{Ni}$ & 0.37 \\
\hline $\mathrm{Cu}$ & 1.7 \\
\hline $\mathrm{Zn}$ & $<0.1$ \\
\hline $\mathrm{Ga}$ & $<0.1$ \\
\hline $\mathrm{Ge}$ & $<0.1$ \\
\hline $\mathrm{As}$ & $<0.1$ \\
\hline $\mathrm{Se}$ & $<0.1$ \\
\hline $\mathrm{Br}$ & $<0.1$ \\
\hline $\mathrm{Rb}$ & $<0.05$ \\
\hline $\mathrm{Sr}$ & 0.19 \\
\hline $\mathrm{Y}$ & 0.95 \\
\hline $\mathrm{Zr}$ & 0.26 \\
\hline $\mathrm{Nb}$ & $<0.1$ \\
\hline $\mathrm{Mo}$ & $<0.05$ \\
\hline $\mathrm{Ru}$ & $<0.1$ \\
\hline $\mathrm{Rh}$ & $<0.1$ \\
\hline & \\
\hline & \\
\hline & \\
\hline & \\
\hline & \\
\hline & \\
\hline
\end{tabular}

P.O. \#

Job \#

Shiva ID:

Element

Concentration

[ ppm wt ]

\begin{tabular}{|c|c|}
\hline$\overline{P d}$ & $<0.1$ \\
\hline $\mathrm{Ag}$ & $<0.1$ \\
\hline $\mathrm{Cd}$ & $<0.1$ \\
\hline $\ln$ & Binder \\
\hline $\mathrm{Sn}$ & $<0.5$ \\
\hline $\mathrm{Sb}$ & $<0.5$ \\
\hline $\mathrm{Te}$ & $<0.1$ \\
\hline 1 & $<0.1$ \\
\hline Cs & $<0.1$ \\
\hline $\mathrm{Ba}$ & 13 \\
\hline $\mathrm{La}$ & $<0.5$ \\
\hline $\mathrm{Ce}$ & 0.08 \\
\hline $\mathrm{Pr}$ & $<0.05$ \\
\hline $\mathrm{Nd}$ & $<0.05$ \\
\hline $\mathrm{Sm}$ & $<0.05$ \\
\hline $\mathrm{Eu}$ & $<0.05$ \\
\hline $\mathrm{Gd}$ & $<0.05$ \\
\hline $\mathrm{Tb}$ & $<0.05$ \\
\hline Dy & $<0.05$ \\
\hline $\mathrm{Ho}$ & $<0.05$ \\
\hline Er & $<0.05$ \\
\hline $\mathrm{Tm}$ & $<0.05$ \\
\hline $\mathrm{Yb}$ & $<0.05$ \\
\hline $\mathrm{Lu}$ & $<0.05$ \\
\hline $\mathrm{Hf}$ & $<0.05$ \\
\hline $\mathrm{Ta}$ & $<5$ \\
\hline $\mathrm{W}$ & 3.1 \\
\hline $\operatorname{Re}$ & $<0.05$ \\
\hline Os & $<0.05$ \\
\hline Ir & $<0.05$ \\
\hline $\mathrm{Pt}$ & $<0.05$ \\
\hline $\mathrm{Au}$ & $<0.1$ \\
\hline $\mathrm{Hg}$ & $<0.5$ \\
\hline $\mathrm{TI}$ & $<0.1$ \\
\hline $\mathrm{Pb}$ & $<0.5$ \\
\hline $\mathrm{Bi}$ & $<0.1$ \\
\hline Th & $<0.05$ \\
\hline $\mathrm{U}$ & $<0.05$ \\
\hline
\end{tabular}

MCH4-0191

UM4335

U031218080 
$\begin{array}{ll}\text { Customer: } & \text { UT-Battelle Oak Ridge } \\ \text { Date: } & \text { 26-Dec-03 }\end{array}$

Customer ID: Graphite

SGL
P.O. \#
Job \#

Shiva ID:

Element

\begin{tabular}{|c|c|c|c|}
\hline $\mathrm{At}$ & [ppm wt ] & $\pi$ & [ppm wt ] \\
\hline $\mathrm{Li}$ & $<0.01$ & $\mathrm{Pd}$ & $<0.1$ \\
\hline $\mathrm{Be}$ & $<0.01$ & $\mathrm{Ag}$ & $<0.1$ \\
\hline$B$ & 2.1 & $\mathrm{Cd}$ & $<0.1$ \\
\hline C & Matrix & $\ln$ & Binder \\
\hline $\mathrm{N}$ & - & $\mathrm{Sn}$ & $<0.5$ \\
\hline 0 & - & $\mathrm{Sb}$ & $<0.5$ \\
\hline $\mathrm{F}$ & $<5$ & $\mathrm{Te}$ & $<0.1$ \\
\hline $\mathrm{Na}$ & 0.45 & 1 & $<0.5$ \\
\hline $\mathrm{Mg}$ & 0.2 & $\mathrm{Cs}$ & $<0.5$ \\
\hline $\mathrm{Al}$ & 0.35 & $\mathrm{Ba}$ & $<0.1$ \\
\hline Si & 3.1 & $\mathrm{La}$ & $<0.5$ \\
\hline $\mathrm{P}$ & 0.11 & $\mathrm{Ce}$ & $<0.05$ \\
\hline$S$ & 9 & $\mathrm{Pr}$ & $<0.05$ \\
\hline $\mathrm{Cl}$ & 3.2 & $\mathrm{Nd}$ & $<0.05$ \\
\hline $\mathrm{K}$ & 0.45 & $\mathrm{Sm}$ & $<0.05$ \\
\hline $\mathrm{Ca}$ & 0.7 & $\mathrm{Eu}$ & $<0.05$ \\
\hline Sc & $<0.05$ & $\mathrm{Gd}$ & $<0.05$ \\
\hline $\mathrm{Ti}$ & 0.06 & $\mathrm{~Tb}$ & $<0.05$ \\
\hline V & 0.02 & Dy & $<0.05$ \\
\hline $\mathrm{Cr}$ & $<0.5$ & $\mathrm{Ho}$ & $<0.05$ \\
\hline $\mathrm{Mn}$ & $<0.05$ & $\mathrm{Er}$ & $<0.05$ \\
\hline $\mathrm{Fe}$ & 1.4 & $\mathrm{Tm}$ & $<0.05$ \\
\hline $\mathrm{Co}$ & 0.25 & $\mathrm{Yb}$ & $<0.05$ \\
\hline $\mathrm{Ni}$ & 1.2 & $\mathrm{Lu}$ & $<0.05$ \\
\hline $\mathrm{Cu}$ & $<0.5$ & $\mathrm{Hf}$ & $<0.05$ \\
\hline $\mathrm{Zn}$ & $<0.5$ & $\mathrm{Ta}$ & $<5$ \\
\hline $\mathrm{Ga}$ & $<0.1$ & W & 2.7 \\
\hline $\mathrm{Ge}$ & $<0.1$ & $\mathrm{Re}$ & $<0.05$ \\
\hline As & $<0.1$ & Os & $<0.05$ \\
\hline $\mathrm{Se}$ & $<0.1$ & $\mathrm{Ir}$ & $<0.05$ \\
\hline $\mathrm{Br}$ & $<0.1$ & $\mathrm{Pt}$ & $<0.05$ \\
\hline $\mathrm{Rb}$ & $<0.05$ & $\mathrm{Au}$ & $<0.1$ \\
\hline $\mathrm{Sr}$ & $<0.05$ & $\mathrm{Hg}$ & $<0.5$ \\
\hline$Y$ & $<0.05$ & $\mathrm{TI}$ & $<0.1$ \\
\hline $\mathrm{Zr}$ & $<0.05$ & $\mathrm{~Pb}$ & $<0.5$ \\
\hline $\mathrm{Nb}$ & $<0.1$ & $\mathrm{Bi}$ & $<0.1$ \\
\hline Mo & $<0.05$ & Th & $<0.05$ \\
\hline $\mathrm{Ru}$ & $<0.1$ & U & $<0.05$ \\
\hline $\mathrm{Rh}$ & $<0.1$ & & \\
\hline
\end{tabular}

Concentration

[ ppm wt ]

Element
MCH4-0191

UM4335

U031218078
Concentration

[ppm wt] 
ORNLETEA+2009/3055] 431-9900

Fax: [315] 431-9800

Email info.ny@eaglabs.com www.eaglabs.com
Customer:
UT-Battelle Oak Ridge
Date:
5-Feb-09
P.O.\#
CC
Job \#
S09N5953
Customer ID: Graphite
Shiva ID:
S090130048
RDKRS 11309

\begin{tabular}{|c|c|c|c|}
\hline Element & $\begin{array}{c}\text { Concentration } \\
{[\mathrm{ppm} \text { wt ] }}\end{array}$ & Element & $\begin{array}{c}\text { Concentration } \\
{[\mathrm{ppm} \text { wt ] }}\end{array}$ \\
\hline $\mathrm{Li}$ & $<0.01$ & $\mathrm{Pd}$ & $<0.1$ \\
\hline $\mathrm{Be}$ & $<0.01$ & $\mathrm{Ag}$ & $<0.1$ \\
\hline$B$ & 0.27 & $\mathrm{Cd}$ & $<0.1$ \\
\hline C & Matrix & $\ln$ & Binder \\
\hline $\mathrm{N}$ & - & $S n$ & $<0.5$ \\
\hline 0 & - & $\mathrm{Sb}$ & $<0.5$ \\
\hline$F$ & $<5$ & $\mathrm{Te}$ & $<0.1$ \\
\hline $\mathrm{Na}$ & 16 & 1 & $<20$ \\
\hline $\mathrm{Mg}$ & $<0.5$ & Cs & $<0.1$ \\
\hline Al & $<0.05$ & $\mathrm{Ba}$ & 4.8 \\
\hline $\mathrm{Si}$ & 12 & $\mathrm{La}$ & $<0.5$ \\
\hline$P$ & 0.12 & $\mathrm{Ce}$ & $<0.5$ \\
\hline$S$ & 5.1 & $\mathrm{Pr}$ & $<0.05$ \\
\hline $\mathrm{Cl}$ & 4.2 & $\mathrm{Nd}$ & $<0.05$ \\
\hline $\mathrm{K}$ & $<0.1$ & $\mathrm{Sm}$ & $<0.05$ \\
\hline $\mathrm{Ca}$ & 0.45 & $\mathrm{Eu}$ & $<0.05$ \\
\hline Sc & $<0.05$ & $\mathrm{Gd}$ & $<0.05$ \\
\hline $\mathrm{Ti}$ & 0.73 & $\mathrm{~Tb}$ & $<0.05$ \\
\hline V & 6.3 & Dy & $<0.05$ \\
\hline $\mathrm{Cr}$ & $<0.5$ & $\mathrm{Ho}$ & $<0.05$ \\
\hline $\mathrm{Mn}$ & $<0.05$ & $\mathrm{Er}$ & $<0.05$ \\
\hline $\mathrm{Fe}$ & 0.03 & $\mathrm{Tm}$ & $<0.05$ \\
\hline Co & $<0.05$ & $Y b$ & $<0.05$ \\
\hline $\mathrm{Ni}$ & $<0.1$ & Lu & $<0.05$ \\
\hline $\mathrm{Cu}$ & $<0.1$ & $\mathrm{Hf}$ & $<0.05$ \\
\hline $\mathrm{Zn}$ & 0.31 & $\mathrm{Ta}$ & $<5$ \\
\hline $\mathrm{Ga}$ & $<0.1$ & $\mathrm{~W}$ & $<0.05$ \\
\hline $\mathrm{Ge}$ & $<0.1$ & $\mathrm{Re}$ & $<0.05$ \\
\hline As & $<0.1$ & Os & $<0.05$ \\
\hline $\mathrm{Se}$ & $<0.1$ & Ir & $<0.05$ \\
\hline $\mathrm{Br}$ & $<0.1$ & $\mathrm{Pt}$ & $<0.05$ \\
\hline $\mathrm{Rb}$ & $<0.05$ & $\mathrm{Au}$ & $<0.1$ \\
\hline $\mathrm{Sr}$ & $<0.05$ & $\mathrm{Hq}$ & $<0.5$ \\
\hline $\bar{Y}$ & $<0.05$ & TI & $<0.1$ \\
\hline $\mathrm{Zr}$ & 0.32 & $\mathrm{~Pb}$ & $<0.5$ \\
\hline $\mathrm{Nb}$ & $<0.1$ & $\mathrm{Bi}$ & $<0.1$ \\
\hline Mo & $<0.05$ & Th & $<0.05$ \\
\hline $\mathrm{Ru}$ & $<0.1$ & $U$ & 2.1 \\
\hline $\mathrm{Rh}$ & $<0.1$ & & \\
\hline
\end{tabular}


HE:IION Hexion Specialty Chemicals, Inc. Page 1 of 1

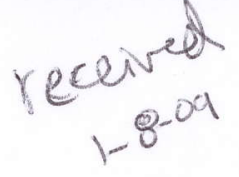

Customer \#: 32710

Customer Address:

CAPITAL RESIN CORPORATION

324 DEFING AVENUE

COLUMBUS OH 43207

USA

$\begin{array}{ll}\text { Ship Date: } & 10 / 29 / 2008 \\ \text { DDN: } & 82444642 \\ \text { Customer - POW: } & 901634 \\ \text { Dote of MFG: } & 10 / 27 / 2008\end{array}$
Product Description: Durite SC-1008
DS3271/450\#
Attention:
KAY FREY
Customer Phone \#: 614-445-7177
Customer Fax \#; $\quad 614-445-7290$
SAP Product : $\quad 359917$

Test Method

\begin{tabular}{|c|c|c|c|c|c|}
\hline Property & Value & Units & Specification F & Reanges & Test Metl \\
\hline \multicolumn{6}{|c|}{ Lot Number: LK8JDO450 } \\
\hline $\mathrm{pH}, 25 \mathrm{C}$ & 8.02 & & 7.90 & 8.50 & IR-034 \\
\hline Specific Gravity & 1.0823 & & 1.0700 & 1.1000 & $1 R=026$ \\
\hline Viscosity & 228 & $\mathrm{cP}_{\mathrm{s}}$ & 180 & 300 & IR.TIT \\
\hline In-process Tests & Passas & - & $n$ & . & \\
\hline Solids. Phenolic (ISO) & 62.20 & $\%$ & 60.00 & 64,00 & IR-063 \\
\hline
\end{tabular}

CERTIFICATE OF COMPLIANCE

It is hereby certified that Hexlon's Phenolic Resin, SC-1008, shipped in this lot has been produced in accordance with Military Specification (Rosin, Phenolle, Laminating) MIL-R9299C, Grades A and B, dated December 3, 7968. It is recommended that SC-7008 be stored in a cool place. Storage life is materially increased by refrigerated storage. $\$ c-1008$ has a usable life of one month at 70 degrees $F$ and six. months ar 40 degrees $F$. 


\section{Characterization of coated particles}

This section contains characterization data on the TRISO particle lot LEU07. The data was obtained according to product inspection plan AGR-CHAR-PIP-10R2, "Product Inspection Plan for AGR-2 Particles for Compacting - Preliminary Measurements". The data obtained by this inspection plan is used in support of compact fabrication and for input into measurements made for compact acceptance testing. There are no direct specifications for the measured parameters.

After washing, the G73J-14-93072A particles were renamed LEU07 and AGR-CHAR-PIP-10R2 was completed. This plan calls for measurement of average particle weight, diameter, envelope volume, and uranium content. OPyC open porosity is also obtained as part of the envelope volume analysis and reported for information only. The plan also calls for riffling of 20 gram aliquots for use as overcoater charges. Riffling at ORNL was done using a 10 position rotary riffler. After riffling out the characterization samples, forty-eight overcoater charges were prepared and labeled LEU07-Y01 through Y48. Additional ORNL characterization performed on another sample taken from G73J-14-93072A is provided in ORNL/TM-2008/133, "Data Compilation for AGR-2 UCO Baseline Coated Particle Batch G73J-14-93072A".

The following pages show the inspection report form (IRF-10) for the LEU07 particles. Following the IRF-10 inspection report form, which summarizes the results, are the individual data report forms for the measurements that were performed. 


\section{Inspection Report Form IRF-10: AGR-2 Particles for Compacting - Preliminary Measurements}

Procedure: |AGR-CHAR-PIP-10 Rev. 2

Coated particle composite ID: LEU07

Coated particle composite description: AGR-2 UCO Baseline, from G73J-14-93072A

\begin{tabular}{|c|c|c|c|c|c|c|c|c|c|}
\hline \multirow[b]{2}{*}{ Property } & \multicolumn{4}{|c|}{ Measured Data } & \multirow{2}{*}{$\begin{array}{l}\text { Specification } \\
\text { INL SPC-923 } \\
\end{array}$} & \multirow[b]{2}{*}{ Acceptance Criteria } & \multirow{2}{*}{$\begin{array}{l}\text { Acceptance } \\
\text { Test Value }\end{array}$} & \multirow{2}{*}{$\begin{array}{l}\text { Pass } \\
\text { or } \\
\text { fail } \\
\end{array}$} & \multirow{2}{*}{$\begin{array}{c}\text { Data } \\
\text { Records }\end{array}$} \\
\hline & $\begin{array}{l}\text { Mean } \\
(x)\end{array}$ & $\begin{array}{l}\text { Std. Dev. } \\
\text { (s) }\end{array}$ & $\begin{array}{c}\# \text { measured } \\
(n)\end{array}$ & t value & & & & & \\
\hline Particle diameter $(\mu \mathrm{m})$ & 861.8 & 24 & 2174 & 1.646 & \multicolumn{4}{|c|}{ Not Applicable } & $\begin{array}{l}\text { DRF-07 } \\
\text { DRF-10 }\end{array}$ \\
\hline Average particle weight $(g)$ & $1.004 E-03$ & & & & \multicolumn{4}{|c|}{ Not Applicable } & DRF-22 \\
\hline Average particle envelope volume $\left(\mathrm{cm}^{3}\right)$ & $3.29 \mathrm{E}-04$ & & & & \multicolumn{4}{|c|}{ Not Applicable } & DRF-31 \\
\hline OPyC open porosity $\left(\mathrm{ml} / \mathrm{m}^{2}\right)$ & 0.400 & & & & \multicolumn{4}{|c|}{ Not Applicable } & DRF-31 \\
\hline Average uranium per particle $(g)$ & $3.93 E-04$ & & & & \multicolumn{4}{|c|}{ Not Applicable } & DRF-35 \\
\hline
\end{tabular}

Comments

Particle weight from combined results of 2 independent measurements (W09010801 and W09011403).
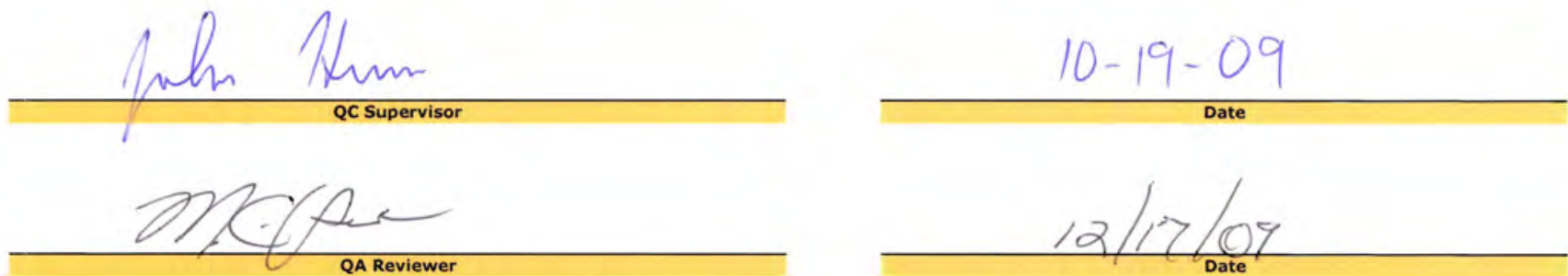
Data Report Form DRF-07: Imaging of Particle Diameter and Aspect Ratio Using an Optical Microscope System

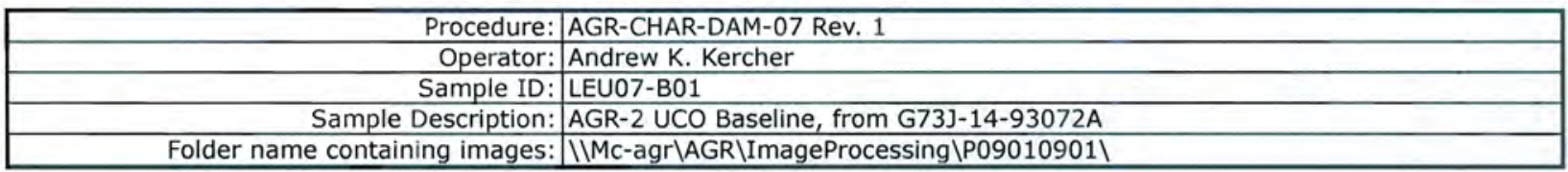

DMR Calibration Expiration Date: $10 / 28 / 09$

Stage Micrometer Calibration Expiration Date: 2/13/09

Measured Value for $1200 \mu \mathrm{m}$ in Stage Micrometer Image: $1200.9 \mu \mathrm{m}$
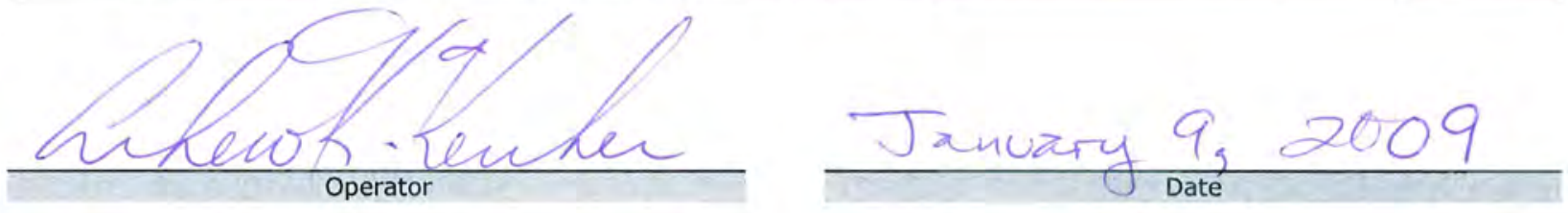


\section{Data Report Form DRF-10A: Measurement of Particle Diameter}

\begin{tabular}{|c|c|}
\hline Procedure: & AGR-CHAR-DAM-10 Rev. 2 \\
\hline Operator: & Andrew K. Kercher \\
\hline Folder name containing images: & 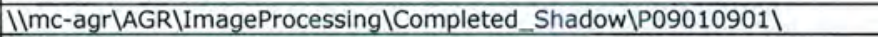 \\
\hline Sample ID: & LEU07-B01 \\
\hline Sample Description: & AGR-2 UCO Baseline, from G73]-14-93072A \\
\hline Folder name containing processed data: & \Imc-agr\AGR\ImageProcessing\Completed_Shadow\P09010901_output\ \\
\hline
\end{tabular}

Number of particles analyzed:

Mean of the average diameter of each particle $(\mu \mathrm{m})$ :

Standard deviation in the average diameter of each particle $(\mu \mathrm{m})$ :

2174

Distribution of the average particle diameter (top binned)

\begin{tabular}{|c|c|}
\hline Mean Diameter $(\mu \mathrm{m})$ & Frequency \\
\hline \hline$<790$ & 0 \\
\hline 800 & 4 \\
\hline 810 & 23 \\
\hline 820 & 44 \\
\hline 830 & 121 \\
\hline 840 & 209 \\
\hline 850 & 269 \\
\hline 860 & 377 \\
\hline 870 & 364 \\
\hline 880 & 279 \\
\hline 890 & 216 \\
\hline 900 & 138 \\
\hline 910 & 76 \\
\hline 920 & 39 \\
\hline 930 & 12 \\
\hline 940 & 3 \\
\hline$>940$ & 0 \\
\hline
\end{tabular}
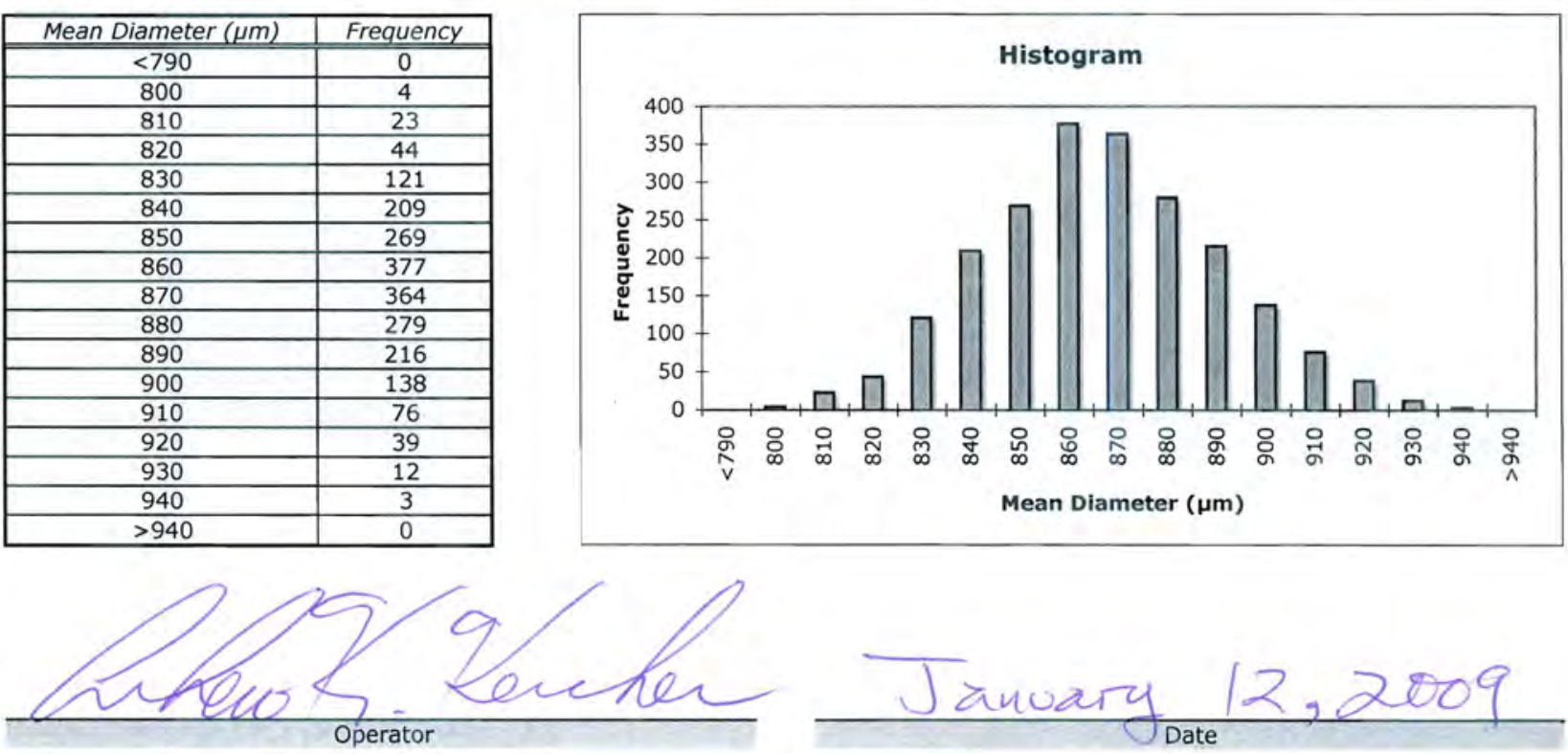
Data Report Form DRF-10B: Measurement of Particle Aspect Ratio (Dmax/Dmin)

\begin{tabular}{|c|c|}
\hline Procedure: & AGR-CHAR-DAM-10 Rev. 2 \\
\hline Operator: & Andrew K. Kercher \\
\hline Folder name containing images: & IImc-agr\AGR\ImageProcessing\Completed_Shadow\P09010901\ \\
\hline Sample ID: & LEU07-B01 \\
\hline Sample Description: & AGR-2 UCO Baseline, from G73]-14-93072A \\
\hline older name containing processed data: & 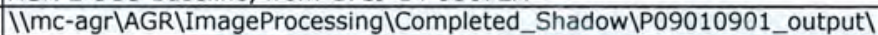 \\
\hline
\end{tabular}

\begin{tabular}{|r|r|}
\hline Number of particles analyzed: & 2174 \\
\hline Number of particles with aspect ratio $\geq 1.14$ & 0 \\
\hline Average particle aspect ratio: & 1.053 \\
\hline
\end{tabular}

Distribution of the aspect ratio (top binned)

\begin{tabular}{|c|c|}
\hline Aspect Ratio (D) & Frequency \\
\hline 1.005 & 0 \\
\hline 1.010 & 0 \\
\hline 1.015 & 4 \\
\hline 1.020 & 15 \\
\hline 1.025 & 39 \\
\hline 1.030 & 97 \\
\hline 1.035 & 134 \\
\hline 1.040 & 189 \\
\hline 1.045 & 246 \\
\hline 1.050 & 266 \\
\hline 1.055 & 261 \\
\hline 1.060 & 249 \\
\hline 1.065 & 162 \\
\hline 1.070 & 165 \\
\hline 1.075 & 116 \\
\hline 1.080 & 81 \\
\hline 1.085 & 65 \\
\hline 1.090 & 37 \\
\hline 1.095 & 22 \\
\hline 1.100 & 12 \\
\hline 1.105 & 6 \\
\hline 1.110 & 4 \\
\hline 1.115 & 2 \\
\hline 1.120 & 1 \\
\hline 1.125 & 0 \\
\hline 1.130 & 0 \\
\hline 1.135 & 1 \\
\hline 1.140 & 0 \\
\hline$>1.14$ & 0 \\
\hline
\end{tabular}
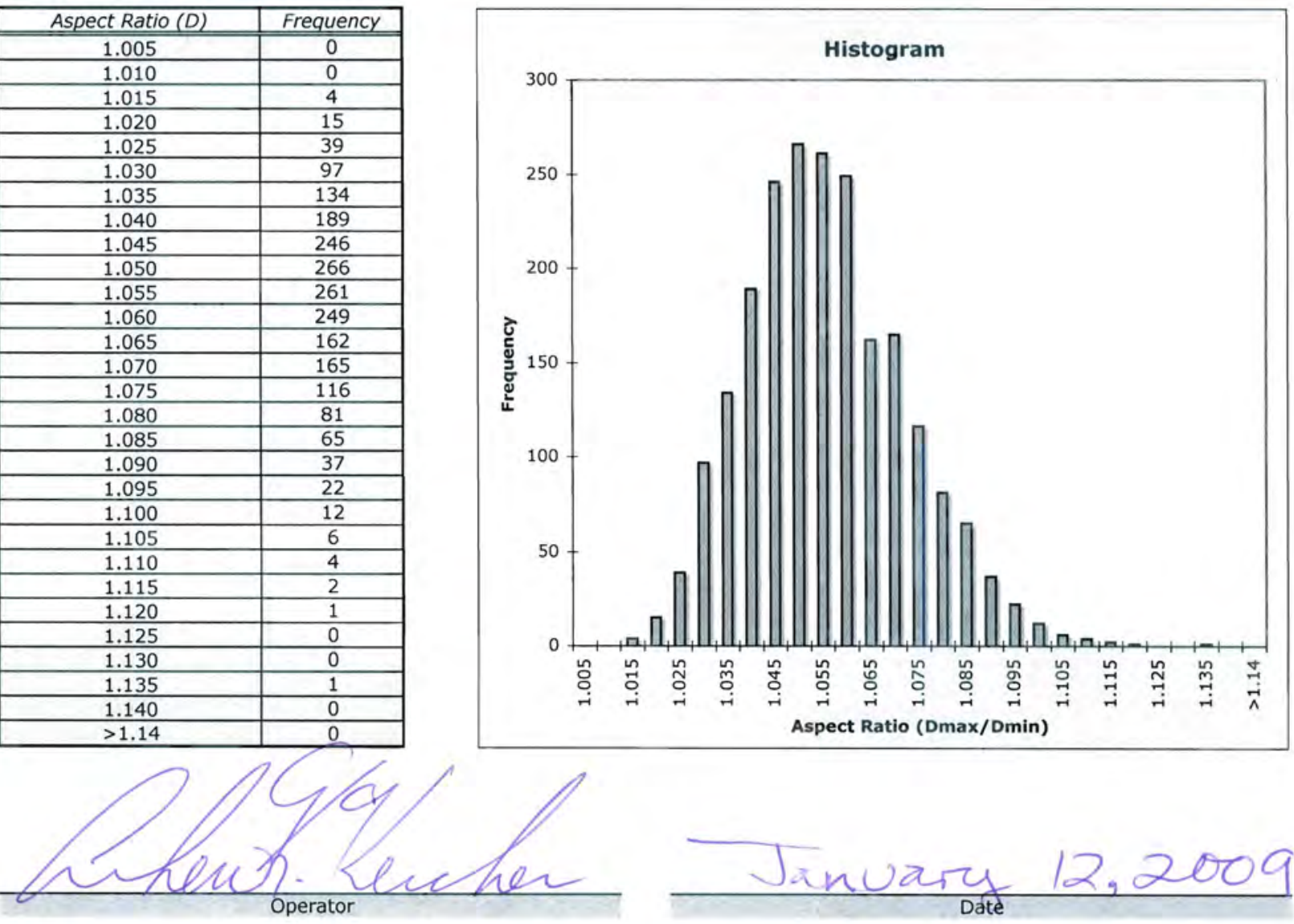
Data Report Form DRF-22: Estimation of Average Particle Weight

\begin{tabular}{|r|l|}
\hline Procedure: & AGR-CHAR-DAM-22 Rev. 1 \\
\hline Operator: & Dixie Barker \\
\hline Particle Lot ID: & LEU07 \\
\hline Particle Lot Description: & AGR-2 UCO Baseline, from G73J-14-93072A \\
\hline Filename: & \ImC-agr\AGR\ParticleWeight \W09010801_DRF22R1.xIs \\
\hline
\end{tabular}

\begin{tabular}{|r|c|c|c|c|c|}
\hline & Sample 1 & Sample 2 & Sample 3 & Sample 4 & Sample 5 \\
\hline Weight of particles (g): & 0.1174 & 0.1920 & 0.2865 & 0.3047 & 0.2650 \\
\hline Number of particles: & 118 & 191 & 284 & 303 & 265 \\
\hline Average weight/particle (g): & $9.949 \mathrm{E}-04$ & $1.005 \mathrm{E}-03$ & $1.009 \mathrm{E}-03$ & $1.006 \mathrm{E}-03$ & $1.000 \mathrm{E}-03$ \\
\hline
\end{tabular}

Mean average weight/particle (g): $1.003 \mathrm{E}-03$

Standard error in mean average weight/particle $(\mathrm{g}): 2.45 \mathrm{E}-06$
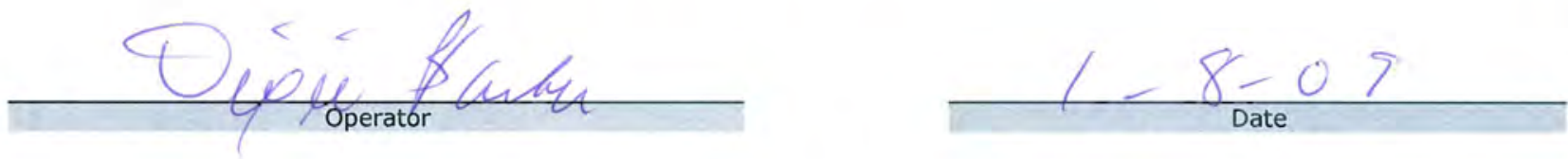
Data Report Form DRF-22: Estimation of Average Particle Weight

\begin{tabular}{|c|c|c|c|c|c|}
\hline & Procedure: & \multicolumn{4}{|c|}{ AGR-CHAR-DAM-22 Rev. 1} \\
\hline & Operator: & \multicolumn{4}{|c|}{ Dixie Barker } \\
\hline & Particle Lot ID: & \multicolumn{4}{|l|}{ LEU07 } \\
\hline Particle & Lot Description: & \multicolumn{4}{|c|}{ AGR-2 UCO Baseline, from G73J-14-93072A } \\
\hline & Filename: & \multicolumn{4}{|c|}{ \Imc-agr|AGR\ParticleWeight|W09011403_DRF22R1.xls } \\
\hline & Sample 6 & Sample 7 & Sample 8 & Sample 9 & Sample 10 \\
\hline Weight of particles $(\mathrm{g})$ : & 0.2836 & 0.1488 & 0.1596 & 0.1329 & 0.1461 \\
\hline Number of particles: & 282 & 148 & 160 & 132 & 145 \\
\hline Average weight/particle $(\mathrm{g})$ : & $1.006 \mathrm{E}-03$ & 1.005E-03 & 9.975E-04 & 1.007E-03 & $1.008 \mathrm{E}-03$ \\
\hline
\end{tabular}

Mean average weight/particle $(\mathrm{g}): 1.005 \mathrm{E}-03$

Standard error in mean average weight/particle $(\mathrm{g}): 1.82 \mathrm{E}-06$
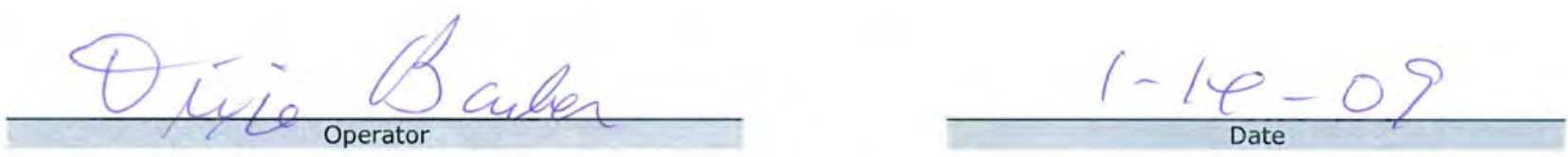
Data Report Form DRF-31: Measurement of Open Porosity using a Mercury Porosimeter

\begin{tabular}{|c|c|}
\hline Procedure: & AGR-CHAR-DAM-31 Rev. 1 \\
\hline Operator: & S. D. Nunn \\
\hline Coated particle batch ID: & LEU07-D01 \\
\hline Batch Description: & AGR-2 UCO Baseline, from G73J-14-93072A \\
\hline Thermocouple Expiration Date: & $5 / 15 / 09$ \\
\hline Penetrometer Expiration Date: & $7 / 10 / 09$ \\
\hline Completed DRF Filename: & IImc-agr|AGR\Porosimeter|S09012201\S09012201_DRF31R1.xls \\
\hline
\end{tabular}

Mean average weight/particle $(g): 1.004 \mathrm{E}-03$

Standard error in mean average weight/particle $(\mathrm{g}): 1.5 \mathrm{E}-06$

\begin{tabular}{|r|l|}
\hline Weight of particles $(\mathrm{g}):$ & 3.9258 \\
\hline Approximate number of particles: & 3910 \\
\hline Uncertainty in number of particles: & 6 \\
\hline Total envelope volume of sample $(\mathrm{cc}):$ & 1.285 \\
\hline Average envelope volume/particle $(\mathrm{cc}):$ & $3.29 \mathrm{E}-04$ \\
\hline Sample envelope density $(\mathrm{g} / \mathrm{cc}):$ & 3.055 \\
\hline &
\end{tabular}

\begin{tabular}{|r|l|}
\hline Average particle diameter (microns): & $8.56 \mathrm{E}+02$ \\
\hline Average surface area/particle $(\mathrm{cm} 2):$ & $2.30 \mathrm{E}-02$ \\
\hline Total sample surface area $(\mathrm{cm} 2):$ & $9.01 \mathrm{E}+01$ \\
\hline Open porosity $(\mathrm{ml} / \mathrm{ml} 2):$ & $3.60 \mathrm{E}-03$ \\
\hline Intruded mercury volume from $250-10,000 \mathrm{p}-01$ \\
\hline
\end{tabular}


Data Report Form DRF-35: Fuel Particle Uranium Loading

\begin{tabular}{|r|l|}
\hline Procedure: & AGR-CHAR-DAM-35 Rev. 0 \\
\hline Operator: & Fred Móntgomery \\
\hline Particle lot ID: & LEU07 \\
\hline Particle lot description: & AGR-2 UCO Baseline, from G73J-14-93072A \\
\hline Filename: & IIMC-agr|AGR\UraniumLoading LLEU07_DRF35R0.xIS \\
\hline
\end{tabular}

Mean average weight per particle $(\mathrm{g}): 1.004 \mathrm{E}-03$

Standard error in mean average weight per particle $(\mathrm{g}): 1.5 \mathrm{E}-06$

\begin{tabular}{|c|c|c|c|c|c|c|}
\hline & \multicolumn{2}{|c|}{ Sample 1 } & \multicolumn{2}{|c|}{ Sample 2} & \multicolumn{2}{|c|}{ Sample 3} \\
\hline & Leach 1 & Leach 2 & Leach 1 & Leach 2 & Leach 1 & Leach 2 \\
\hline Particle sample ID: & \multicolumn{2}{|c|}{ LEU07-E01 } & \multicolumn{2}{|c|}{ LEU07-F01 } & \multicolumn{2}{|c|}{ LEU07-B01 } \\
\hline Weight of particles: & \multicolumn{2}{|c|}{3.8098} & \multicolumn{2}{|c|}{3.9149} & \multicolumn{2}{|c|}{4.0143} \\
\hline Approximate number of particles: & \multicolumn{2}{|c|}{3795} & \multicolumn{2}{|c|}{3899} & \multicolumn{2}{|c|}{3998} \\
\hline Uncertainty in number of particles: & \multicolumn{2}{|c|}{6} & \multicolumn{2}{|c|}{6} & \multicolumn{2}{|c|}{6} \\
\hline Acid leach sample ID: & U09012101 & U09012201 & U09012102 & U09012202 & U09012104 & U09012204 \\
\hline Radiochemical laboratory analysis number: & $1658-001$ & $1658-005$ & $1658-002$ & $1658-006$ & $1658-004$ & $1658-008$ \\
\hline Weight $U$ in leach $(\mathrm{mg})$ : & 1496 & 0.073 & 1529 & 0.360 & 1569 & 0.370 \\
\hline Uncertainty in weight $U$ in leach $(\mathrm{mg})$ : & 6.0 & 0.0073 & 6.1 & 0.036 & 6.3 & 0.037 \\
\hline Total weight $\mathrm{U}$ in sample $(\mathrm{mg})$ : & \multicolumn{2}{|c|}{1496} & \multicolumn{2}{|c|}{1529} & \multicolumn{2}{|c|}{1569} \\
\hline Average weight $U$ per particle $(\mathrm{mg})$ : & \multicolumn{2}{|c|}{0.3943} & \multicolumn{2}{|c|}{0.3922} & \multicolumn{2}{|c|}{0.3924} \\
\hline Uncertainty in average weight U per particle $(\mathrm{mg}):$ & \multicolumn{2}{|c|}{0.0017} & \multicolumn{2}{|c|}{0.0017} & \multicolumn{2}{|c|}{0.0017} \\
\hline
\end{tabular}

Mean average uranium loading per particle $(\mathrm{g}): 3.930 \mathrm{E}-04$

Standard error in mean average uranium loading per particle $(\mathrm{g}): 6.6 \mathrm{E}-07$

\section{Comments}

Leach 1 was analyzed by Davies-Gray titration method. Leach 2 was analyzed by ICP-MS, due to low U concentration.

A small amount of the crushed particle residue was lost during analysis of LEU07-G01 while removing the tape from the spex mill bottle. LEU07-B01 was analyzed in place of LEU07-G01 as sample \#3.

Particle weight from combined results of 2 independent measurements (W09010801 and W09011403).

Davies-Gray Initial known U recovery: $100.60 \%$; final known U recovery $100.20 \%$ Blind titration U recovery $100.4 \%$.

Uncertainty in Davies-Gray $(0.4 \%)$ based on average of measured \% recovery data for LEU06,07,08,09.

Data checked against official results of analyses for RMAL1658 on 5/26/2009 by FCM 


\section{Characterization of overcoated particles}

This section contains characterization data on the overcoated particle lot LEU07-OP1. The data was obtained according to product inspection plan AGR-CHAR-PIP-11R0, "Product Inspection Plan for AGR-2 Overcoated Particles for Compacting". The data obtained by this inspection plan is used in support of compact fabrication and for input into measurements made for compact acceptance testing. There are no direct specifications for the measured parameters.

After overcoating, the overcoated particles from LEU07-Y01 through Y48 were combined and homogenized into an overcoated particle composite. The overcoated particle composite was labeled LEU07-OP1 and AGR-CHAR-PIP-11R0 was completed. This plan calls for measurement of average overcoated particle weight and diameter. The plan also calls for riffling of compact charges for pressing. One hundred and eighty compact charges were prepared and labeled LEU07-OP1-G001 through G180. A record of the weight of each compact charge can be found on data report form DRF-24D, in section 7.

The following pages show the inspection report form (IRF-11) for the LEU07-OP1 overcoated particles. Following the IRF-11 inspection report form, which summarizes the results, are the individual data report forms for the measurements that were performed.

The average thickness of the overcoat can be estimated from the increase in the average particle size after overcoating, $(1332 \mu \mathrm{m}-862 \mu \mathrm{m}) / 2=235 \mu \mathrm{m}$. The increase in average particle weight was $(2.018 \mathrm{mg}-1.004 \mathrm{mg})=1.014 \mathrm{mg}$. From these values, the average density of the overcoating prior to compacting can be estimated to be $1.12 \mathrm{~g} / \mathrm{cm}^{3}$. 
ORNL/TM-2009/305

Inspection Report Form IRF-11: AGR-2 Overcoated Particles for Compacting

Procedure: AGR-CHAR-PIP-11 Rev, 0

Overcoated particle composite ID: LEU07-OP1

Overcoated particle composite description: AGR -2 UCO Baseline, from G73J-14-93072A

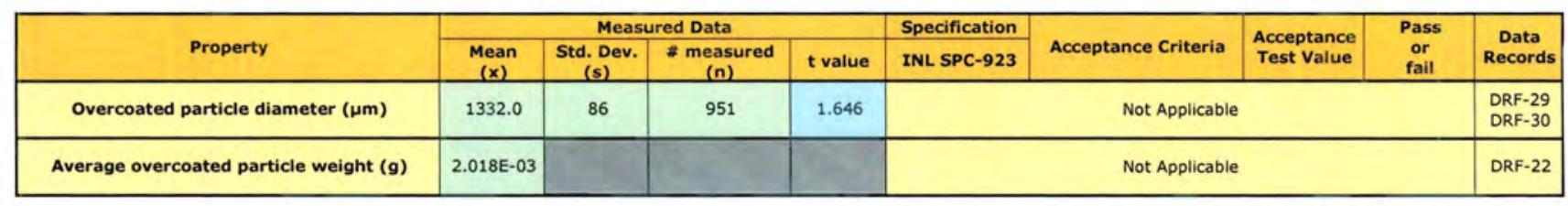

Comments
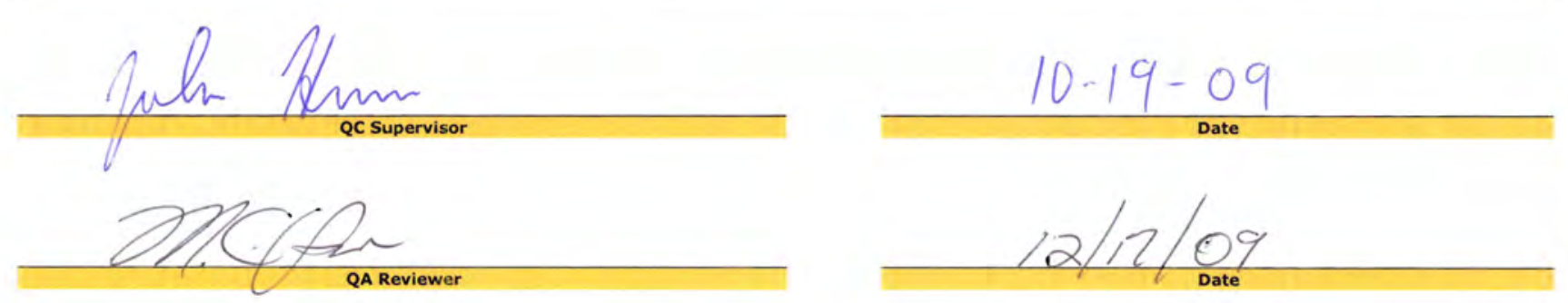

35 


\section{Data Report Form DRF-29: Imaging of Overcoated Particle Diameter and Aspect Ratio Using an Optical Microscope System}

Procedure: AGR-CHAR-DAM-29 Rev. 1

Operator: Andrew K. Kercher

Sample ID: LEU07-OP1-B01

Sample Description: AGR-2 UCO Baseline, from G73J-14-93072A

Folder name containing images: \\mc-agr|AGR\ImageProcessing\P09022001\

DMR Calibration Expiration Date: $10 / 28 / 2009$

Stage Micrometer Calibration Expiration Date: $2 / 10 / 2014$

Measured Value for $2500 \mu \mathrm{m}$ in Stage Micrometer Image: $2501.8 \mu \mathrm{m}$

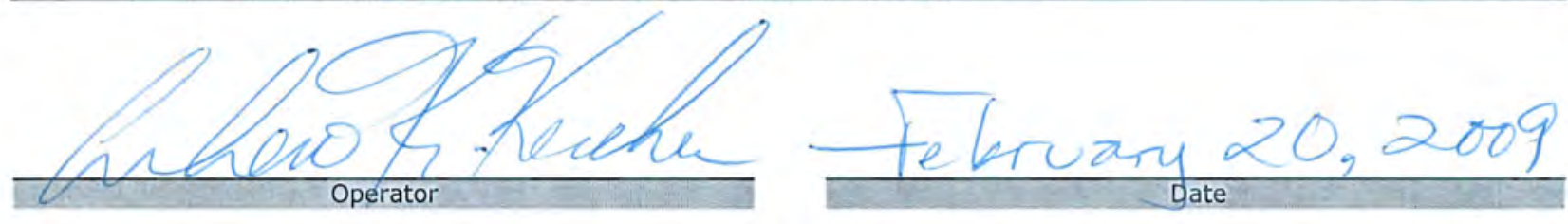


Data Report Form DRF-30A: Measurement of Over-coated Particle Diameter

\begin{tabular}{|c|c|}
\hline Procedure: & AGR-CHAR-DAM-30 Rev. 0 \\
\hline Operator: & Andrew K. Kercher \\
\hline Folder name containing images: & 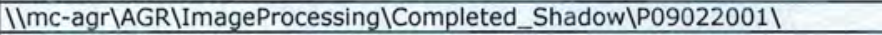 \\
\hline Sample ID: & LEU07-OP1-B01 \\
\hline Sample Description: & AGR-2 UCO Baseline, from G73J-14-93072A \\
\hline
\end{tabular}

\begin{tabular}{r|r|}
\hline Number of particles analyzed: & 951 \\
\hline Mean of the average diameter of each particle $(\mu \mathrm{m}):$ & 1332.0 \\
\hline Standard deviation in the average diameter of each particle $(\mu \mathrm{m}):$ & 86 \\
\hline
\end{tabular}

\section{Distribution of the average particle diameter (top binned)}

\begin{tabular}{|c|c|}
\hline Mean Diameter $(\mu \mathrm{m})$ & Frequency \\
\hline \hline$<1130$ & 2 \\
\hline 1170 & 6 \\
\hline 1210 & 32 \\
\hline 1250 & 97 \\
\hline 1290 & 173 \\
\hline 1330 & 218 \\
\hline 1370 & 163 \\
\hline 1410 & 105 \\
\hline 1450 & 77 \\
\hline 1490 & 32 \\
\hline 1530 & 20 \\
\hline 1570 & 10 \\
\hline 1610 & 6 \\
\hline 1650 & 6 \\
\hline 1690 & 3 \\
\hline 1730 & 1 \\
\hline$>1730$ & 0 \\
\hline &
\end{tabular}
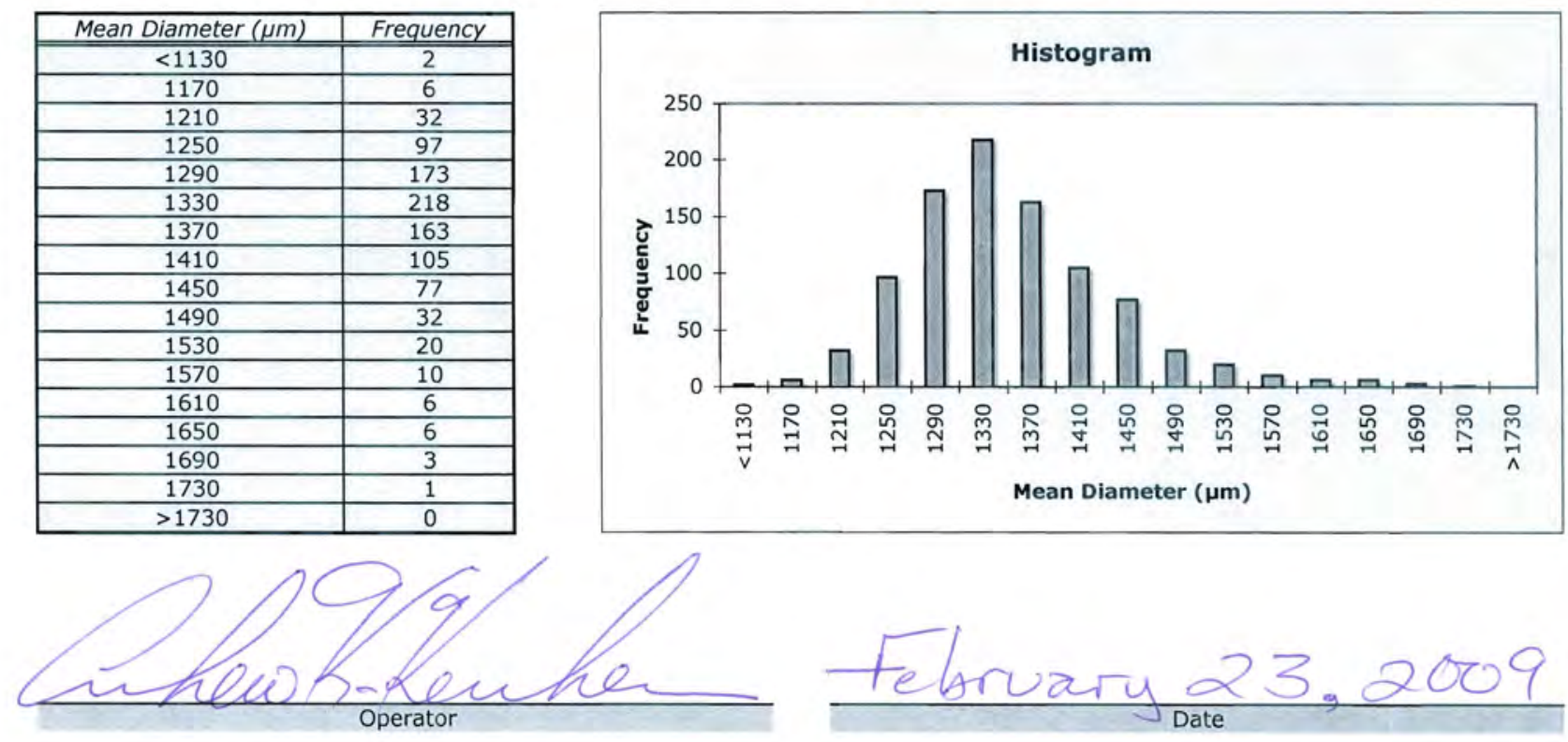
Data Report Form DRF-30B: Measurement of Over-coated Particle Aspect Ratio (Dmax/Dmin)

\begin{tabular}{|c|c|}
\hline Procedure: & AGR-CHAR-DAM-30 Rev. 0 \\
\hline Operator: & Andrew K. Kercher \\
\hline Folder name containing images: & 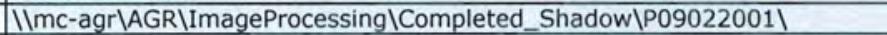 \\
\hline Sample ID: & LEU07-OP1-B01 \\
\hline Sample Description: & AGR-2 UCO Baseline, from G73J-14-93072A \\
\hline Folder name containing processed data: & 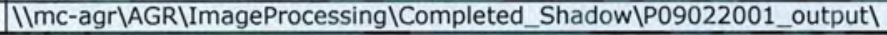 \\
\hline
\end{tabular}

Number of particles analyzed:

951

Average particle aspect ratio:

1.077

\section{Distribution of the aspect ratio (top binned)}

\begin{tabular}{|c|c|}
\hline Aspect Ratio (D) & Frequency \\
\hline 1.005 & 0 \\
\hline 1.010 & 0 \\
\hline 1.015 & 0 \\
\hline 1.020 & 1 \\
\hline 1.025 & 7 \\
\hline 1.030 & 10 \\
\hline 1.035 & 18 \\
\hline 1.040 & 34 \\
\hline 1.045 & 48 \\
\hline 1.050 & 42 \\
\hline 1.055 & 63 \\
\hline 1.060 & 78 \\
\hline 1.065 & 80 \\
\hline 1.070 & 77 \\
\hline 1.075 & 66 \\
\hline 1.080 & 58 \\
\hline 1.085 & 63 \\
\hline 1.090 & 49 \\
\hline 1.095 & 36 \\
\hline 1.100 & 39 \\
\hline 1.105 & 29 \\
\hline 1.110 & 24 \\
\hline 1.115 & 25 \\
\hline 1.120 & 19 \\
\hline 1.125 & 17 \\
\hline 1.130 & 11 \\
\hline 1.135 & 7 \\
\hline 1.140 & 6 \\
\hline$>1.14$ & 44 \\
\hline
\end{tabular}
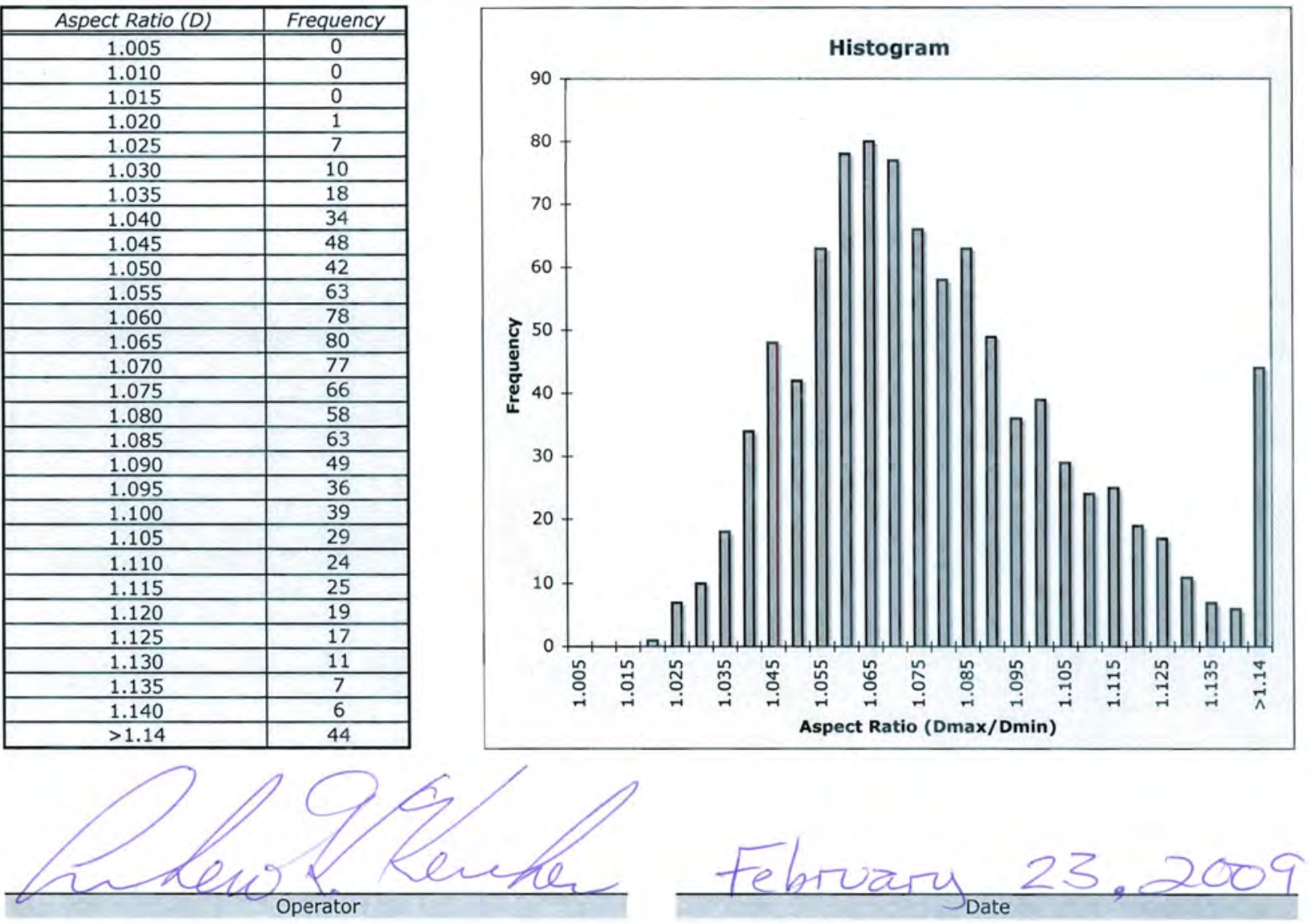
Data Report Form DRF-22: Estimation of Average Particle Weight

Procedure: AGR-CHAR-DAM-22 Rev. 1

Operator: Dixie Barker

Particle Lot ID: LEU07-OP1-C01

Particle Lot Description: AGR-2 UCO Baseline, from G73J-14-93072A

Filename: IImc-agr|AGR\ParticleWeight|W09021901_DRF22R1.xls

\begin{tabular}{|r|c|c|c|c|c|}
\hline & Sample 1 & Sample 2 & Sample 3 & Sample 4 & Sample 5 \\
\hline Weight of particles (g): & 0.2874 & 0.3029 & 0.3274 & 0.3528 & 0.3341 \\
\hline Number of particles: & 142 & 153 & 161 & 174 & 165 \\
\hline Average weight/particle (g): & $2.024 \mathrm{E}-03$ & $1.980 \mathrm{E}-03$ & $2.034 \mathrm{E}-03$ & $2.028 \mathrm{E}-03$ & $2.025 \mathrm{E}-03$ \\
\hline
\end{tabular}

Mean average weight/particle (g): $2.018 \mathrm{E}-03$

Standard error in mean average weight/particle $(\mathrm{g}):$ 9.69E-06

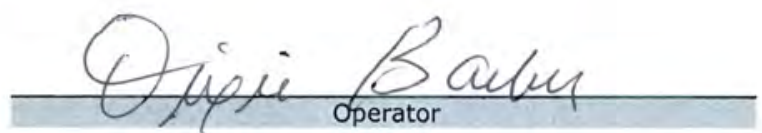

$$
2-19-2009
$$




\section{Characterization of compacts}

This section contains acceptance testing data on the compact lot LEU07-OP1-Z. The data was obtained according to product inspection plan AGR-CHAR-PIP-12R1, "Product Inspection Plan for AGR-2 UCO Fuel Compact Lots". This compact lot was determined to not satisfy the specifications in section 3.3 of the AGR-2 Fuel Specification (INL SPC-923, Rev. 3). The final disposition of this compact lot was to not use for the compacts for the AGR-2 irradiation test, but to retain the compacts in storage at ORNL and INL for possible future analysis or methods development.

After compacting, 155 compacts were selected from LEU07-OP1-G001 through G180 for use. Compacts with obvious processing defects, chips, or undesirable dimensions were sorted out and not included in the 155 compacts selected for the final fuel compact lot. This down-select was part of the compacting process and was performed prior to random selection of compacts for acceptance testing. It should be understood that the results in this section and the acceptance testing are only relevant for the final 155 compact lot from which random representative samples were drawn for characterization. As instructed in AGR-CHAR-PIP-12R1, these 155 compacts were randomized and relabeled as LEU07-OP1-Z001 through Z155. A record of the original Gnumber for each Z-numbered compact can be found on data report form DRF-24C, in this section. After relabeling, the compacts were characterized for product acceptance according to product inspection plan PIP-12. This plan calls for measurement of compact length, diameter, mass, matrix density, uranium content, impurity content, and determination of defect fractions for exposed uranium, defective $\mathrm{SiC}$, uranium dispersion due to defective IPyC, and defective $\mathrm{OPyC}$.

The following pages show the inspection report forms (IRF-12A, IRF-12B, IRF-12C, IRF-12D) for the LEU07-OP1-Z compacts. Following the IRF-12 inspection report forms, which summarize the results, are the individual data report forms for the measurements that were performed. Note that the leach-burn-leach (LBL) analysis is performed on sets of 20 compacts at a time, in four sample groups with 5 compacts in each sample. Inspection report forms IRF-12B, IRF-12C, and IRF-12D summarize the results from each set of 20 compacts. Inspection report form IRF-12A summarizes all the analyses. The mean and standard deviation for the impurity analyses (IRF-12B), the uranium contamination fraction or effective number of exposed kernels before the burn (IRF-12C) and the defective SiC defect fraction or number of exposed kernels after the burn (IRF-12D) are calculated from the combined results of all the relevant sample groups. These combined results, which are then entered into IRF-12A, are provided in Table 7-1 and Table 7-2 below. 
Table 7-1: Summary of impurity analysis for LEU07-OP1-Z compacts

\begin{tabular}{|c|c|c|c|c|c|c|c|c|c|c|c|c|c|c|}
\hline Compact ID numbers: & $\begin{array}{c}060,046, \\
122,146, \\
130\end{array}$ & $\begin{array}{c}141,138, \\
111,064, \\
053\end{array}$ & $\begin{array}{c}023,076, \\
006,040, \\
034\end{array}$ & $\begin{array}{c}139,083, \\
087,145, \\
049\end{array}$ & $\begin{array}{c}005,013, \\
107,058, \\
062\end{array}$ & $\begin{array}{c}135,017, \\
022,018, \\
125\end{array}$ & $\begin{array}{c}037,027, \\
097,025, \\
048\end{array}$ & $\begin{array}{c}129,149, \\
100,029, \\
066\end{array}$ & $\begin{array}{c}055,030 \\
085,108 \\
140\end{array}$ & $\begin{array}{c}026,103, \\
095,119, \\
077\end{array}$ & $\begin{array}{c}112,009, \\
155,056, \\
001\end{array}$ & $\begin{array}{c}144,134, \\
051,115, \\
088\end{array}$ & Mean & $\begin{array}{c}\text { Standard } \\
\text { Deviation }\end{array}$ \\
\hline Number of compacts: & 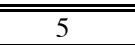 & 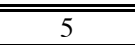 & 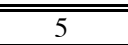 & 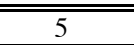 & 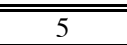 & $\overline{5}$ & 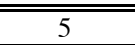 & 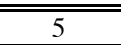 & 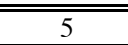 & 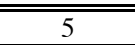 & 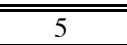 & 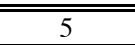 & & \\
\hline \multicolumn{15}{|l|}{$\mathrm{T}$} \\
\hline Deconsolidation-leach (DRF-26A) $(\mu \mathrm{g})$ : & 29.36 & 29.77 & 29.46 & 29.36 & 10.14 & 10.34 & 10.63 & 10.51 & 10.44 & 10.79 & 10.67 & 10.51 & & \\
\hline Burn-leach (DRF-26B) $(\mu \mathrm{g}):$ & 4.65 & 20.29 & 3.10 & 2.99 & 5.67 & 9.44 & 6.63 & 8.28 & 5.65 & 5.97 & 6.63 & 5.76 & & \\
\hline Total leached $(\mu \mathrm{g}):$ & 34.01 & 50.05 & 32.56 & 32.34 & 15.80 & 19.78 & 17.26 & 18.79 & 16.09 & 16.76 & 17.30 & 16.26 & & \\
\hline Fe outside $\mathrm{SiC}(\mu \mathrm{g} / \mathrm{compact}):$ & 6.80 & 10.01 & 6.51 & 6.47 & 3.16 & 3.96 & 3.45 & 3.76 & 3.22 & 3.35 & 3.46 & 3.25 & 4.78 & 2.17 \\
\hline \multicolumn{15}{|l|}{ Chromium } \\
\hline Deconsolidation-leach (DRF-26A) $(\mu \mathrm{g}):$ & 1.79 & 2.03 & 2.43 & 2.17 & 2.21 & 2.71 & 1.90 & 2.47 & 2.33 & 2.11 & 1.86 & 1.90 & & \\
\hline Burn-leach (DRF-26B) $(\mu \mathrm{g}):$ & 0.73 & 0.72 & 0.70 & 0.64 & 0.76 & 0.89 & 0.89 & 0.90 & 0.72 & 0.78 & 0.86 & 0.70 & & \\
\hline Total leached $(\mu \mathrm{g}):$ & 2.52 & 2.74 & 3.13 & 2.81 & 2.97 & 3.60 & 2.79 & 3.38 & 3.04 & 2.89 & 2.72 & 2.60 & & \\
\hline Cr outside $\mathrm{SiC}(\mu \mathrm{g} / \mathrm{compact}):$ & 0.50 & 0.55 & 0.63 & 0.56 & 0.59 & 0.72 & 0.56 & 0.68 & 0.61 & 0.58 & 0.54 & 0.52 & 0.59 & 0.06 \\
\hline \multicolumn{15}{|l|}{ Manganese } \\
\hline Deconsolidation-leach (DRF-26A) $(\mu \mathrm{g})$ : & 1.36 & 1.38 & 1.36 & 1.36 & 0.47 & 0.48 & 0.49 & 0.49 & 0.49 & 0.50 & 0.49 & 0.49 & & \\
\hline Burn-leach (DRF-26B) $(\mu \mathrm{g})$ : & 0.21 & 0.20 & 0.21 & 0.19 & 0.19 & 0.19 & 0.20 & 0.19 & 0.19 & 0.19 & 0.20 & 0.19 & & \\
\hline Total leached $(\mu \mathrm{g})$ : & 1.56 & 1.58 & 1.57 & 1.55 & 0.66 & 0.67 & 0.69 & 0.68 & 0.68 & 0.69 & 0.69 & 0.68 & & \\
\hline Mn outside $\mathrm{SiC}(\mu \mathrm{g} / \mathrm{compact}):$ & 0.31 & 0.32 & 0.31 & 0.31 & 0.13 & 0.13 & 0.14 & 0.14 & 0.14 & 0.14 & 0.14 & 0.14 & 0.20 & 0.09 \\
\hline \multicolumn{15}{|l|}{ 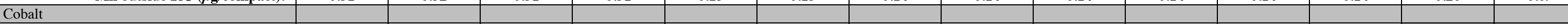 } \\
\hline Deconsolidation-leach (DRF-26A) $(\mu \mathrm{g})$ : & 1.16 & 1.17 & 1.16 & 1.16 & 0.40 & 0.41 & 0.42 & 0.41 & 0.41 & 0.42 & 0.42 & 0.41 & & \\
\hline Burn-leach (DRF-26B) $(\mu \mathrm{g}):$ & 0.09 & 0.09 & 0.09 & 0.09 & 0.16 & 0.16 & 0.17 & 0.16 & 0.16 & 0.16 & 0.17 & 0.16 & & \\
\hline Total leached $(\mu \mathrm{g}):$ & 1.25 & 1.26 & 1.25 & 1.24 & 0.56 & 0.57 & 0.58 & 0.58 & 0.57 & 0.59 & 0.59 & 0.58 & & \\
\hline Co outside $\mathrm{SiC}(\mu \mathrm{g} / \mathrm{compact})$ : & 0.25 & 0.25 & 0.25 & 0.25 & 0.11 & 0.11 & 0.12 & 0.12 & 0.11 & 0.12 & 0.12 & 0.12 & 0.16 & 0.07 \\
\hline \multicolumn{15}{|l|}{ Nickel } \\
\hline Deconsolidation-leach (DRF-26A) $(\mu \mathrm{g}):$ & 5.70 & 5.78 & 5.72 & 5.70 & 1.97 & 2.01 & 2.06 & 2.04 & 2.02 & 2.10 & 2.07 & 2.04 & & \\
\hline Burn-leach (DRF-26B) $(\mu \mathrm{g}):$ & 0.96 & 0.94 & 0.93 & 0.83 & 0.82 & 0.93 & 0.89 & 0.94 & 1.11 & 0.93 & 0.90 & 0.85 & & \\
\hline Total leached $(\mu \mathrm{g}):$ & 6.66 & 6.72 & 6.65 & 6.53 & 2.78 & 2.94 & 2.95 & 2.98 & 3.13 & 3.02 & 2.97 & 2.89 & & \\
\hline Ni outside $\operatorname{SiC}(\mu \mathrm{g} /$ compact): & 1.33 & 1.34 & 1.33 & 1.31 & 0.56 & 0.59 & 0.59 & 0.60 & 0.63 & 0.60 & 0.59 & 0.58 & 0.84 & 0.36 \\
\hline \multicolumn{15}{|l|}{ Transition Metals } \\
\hline $\mathrm{Cr}+\mathrm{Mn}+\mathrm{Co}+\mathrm{Ni}$ outside $\mathrm{SiC}(\mu \mathrm{g} /$ compact $):$ & 2.40 & 2.46 & 2.52 & 2.43 & 1.39 & 1.55 & 1.40 & 1.52 & 1.49 & 1.44 & 1.39 & 1.35 & 1.78 & 0.50 \\
\hline \multicolumn{15}{|l|}{ Calcium } \\
\hline Deconsolidation-leach (DRF-26A) $(\mu \mathrm{g}):$ & 67.14 & 74.30 & 124.36 & 74.38 & 24.60 & 37.31 & 25.80 & 25.50 & 45.30 & 39.18 & 49.14 & 25.50 & & \\
\hline Burn-leach (DRF-26B) $(\mu \mathrm{g}):$ & 118.70 & 121.03 & 112.44 & 102.32 & 136.72 & 160.04 & 167.65 & 199.10 & 109.74 & 116.39 & 90.73 & 115.81 & & \\
\hline Total leached $(\mu \mathrm{g})$ : & 185.84 & 195.33 & 236.81 & 176.70 & 161.32 & 197.35 & 193.45 & 224.60 & 155.04 & 155.57 & 139.87 & 141.31 & & \\
\hline Ca outside $\mathrm{SiC}(\mu \mathrm{g} / \mathrm{compact}):$ & 37.17 & 39.07 & 47.36 & 35.34 & 32.26 & 39.47 & 38.69 & 44.92 & 31.01 & 31.11 & 27.97 & 28.26 & 36.05 & 6.22 \\
\hline \multicolumn{15}{|l|}{ Aluminum } \\
\hline Deconsolidation-leach (DRF-26A) $(\mu \mathrm{g}):$ & 74.72 & 84.31 & 63.69 & 72.66 & 235.12 & 75.87 & 63.69 & 71.66 & 71.17 & 64.86 & 56.07 & 63.95 & & \\
\hline Burn-leach (DRF-26B) $(\mu \mathrm{g})$ : & 73.10 & 71.55 & 73.81 & 71.17 & 63.01 & 66.18 & 65.94 & 67.21 & 60.71 & 74.56 & 62.78 & 57.00 & & \\
\hline Total leached $(\mu \mathrm{g}):$ & 147.82 & 155.87 & 137.50 & 143.83 & 298.13 & 142.05 & 129.63 & 138.87 & 131.89 & 139.42 & 118.85 & 120.96 & & \\
\hline Al outside $\operatorname{SiC}(\mu \mathrm{g} /$ compact): & 29.56 & 31.17 & 27.50 & 28.77 & 59.63 & 28.41 & 25.93 & 27.77 & 26.38 & 27.88 & 23.77 & 24.19 & 30.08 & 9.54 \\
\hline \multicolumn{15}{|l|}{ Titanium } \\
\hline Deconsolidation-leach (DRF-26A) $(\mu \mathrm{g})$ : & 6.05 & 7.72 & 7.78 & 8.23 & 6.75 & 9.00 & 6.76 & 9.90 & 7.65 & 7.17 & 5.31 & 6.62 & & \\
\hline Burn-leach $(\mathrm{DRF}-26 \mathrm{~B})(\mu \mathrm{g})$ & 8.87 & 7.48 & 10.06 & 7.08 & 7.42 & 5.70 & 8.12 & 5.82 & 7.95 & 8.20 & 9.06 & 8.23 & & \\
\hline Total leached $(\mu \mathrm{g}):$ & 14.92 & 15.20 & 17.84 & 15.31 & 14.17 & 14.70 & 14.88 & 15.72 & 15.60 & 15.37 & 14.37 & 14.85 & & \\
\hline Ti outside $\mathrm{SiC}(\mu \mathrm{g} /$ compact): & 2.98 & 3.04 & 3.57 & 3.06 & 2.83 & 2.94 & 2.98 & 3.14 & 3.12 & 3.07 & 2.87 & 2.97 & 3.05 & 0.19 \\
\hline \multicolumn{15}{|l|}{ Vanadium } \\
\hline Deconsolidation-leach (DRF-26A) $(\mu \mathrm{g}):$ & 40.25 & 40.05 & 37.61 & 42.69 & 38.05 & 44.71 & 37.05 & 41.40 & 42.77 & 42.07 & 39.93 & 38.82 & & \\
\hline Burn-leach (DRF-26B) $(\mu \mathrm{g}):$ & 53.93 & 53.39 & 54.92 & 53.74 & 48.96 & 50.11 & 46.19 & 46.00 & 49.25 & 55.51 & 52.53 & 43.79 & & \\
\hline Total leached $(\mu \mathrm{g}):$ & 94.19 & 93.44 & 92.53 & 96.43 & 87.01 & 94.82 & 83.24 & 87.40 & 92.02 & 97.58 & 92.46 & 82.61 & & \\
\hline V outside SiC ( $\mu \mathrm{g} / \mathrm{compact}):$ & 18.84 & 18.69 & 18.51 & 19.29 & 17.40 & 18.96 & 16.65 & 17.48 & 18.40 & 19.52 & 18.49 & 16.52 & 18.23 & 0.99 \\
\hline \multicolumn{15}{|l|}{ Titanium and Vanadium } \\
\hline $\mathrm{Ti}+\mathrm{V}$ outside $\mathrm{SiC}(\mu \mathrm{g} /$ compact $):$ & 21.82 & 21.73 & 22.07 & 22.35 & 20.24 & 21.90 & 19.62 & 20.62 & 21.52 & 22.59 & 21.37 & 19.49 & 21.28 & 1.04 \\
\hline
\end{tabular}


Table 7-2: Summary of uranium contamination and SiC defect analysis for LEU07-OP1-Z compacts

\begin{tabular}{|c|c|c|c|}
\hline Compact ID numbers & Number of compacts & $\begin{array}{c}\text { Effective number of exposed } \\
\text { kernels before burn }\end{array}$ & $\begin{array}{c}\text { Number of kernels } \\
\text { leached after burn }\end{array}$ \\
\hline \hline $060,046,122,146,130$ & 5 & 1.1 & 0 \\
\hline $141,138,111,064,053$ & 5 & 1.1 & 0 \\
\hline $023,076,006,040,034$ & 5 & 0.0 & 0 \\
\hline $139,083,087,145,049$ & 5 & 0.6 & 0 \\
\hline $123,147,152,035,010$ & 5 & 0.0 & 0 \\
\hline $110,045,042,002,086$ & 5 & 2.0 & 0 \\
\hline $136,078,052,070,059$ & 5 & 1.1 & 0 \\
\hline $033,044,124,075,032$ & 5 & 0.2 & 0 \\
\hline $005,013,107,058,062$ & 5 & 2.0 & 0 \\
\hline $135,017,022,018,125$ & 5 & 0.0 & 0 \\
\hline $037,027,097,025,048$ & 5 & 0.0 & 0 \\
\hline $129,149,100,029,066$ & 5 & 1.0 & 0 \\
\hline $055,030,085,108,140$ & 5 & 1.1 & 0 \\
\hline $026,103,095,119,077$ & 5 & 1.1 & 0 \\
\hline $112,009,155,056,001$ & 5 & 0.0 & 0 \\
\hline $144,134,051,115,088$ & 5 & 0.0 & 0 \\
\hline $142,047,011,092,038$ & 5 & 0.0 & 0 \\
\hline $069,050,039,150,028$ & 5 & 0.0 & 0 \\
\hline $114,133,127,091,061$ & 5 & 2.3 & $\mathbf{0}$ \\
\hline $099,024,031,104,101$ & 5 & 1.0 & $\mathbf{1 4}$ \\
\hline \hline Total: & $\mathbf{1 0 0}$ & & \\
\hline
\end{tabular}

One compact was measured to be $0.046 \mathrm{~mm}$ longer than the specified $25.40 \mathrm{~mm}$ upper limit for compact length. This minor deviation is not expected to affect the compact performance and was not associated by significant deviations in the compacting force or matrix density. It was therefore determined that the compact could be used as is. This was documented on ORNL nonconformance report NCR-X-MSTD-AGR-10-01.

After compacts were electrolytically deconsolidated and leached, uranium was detected at a level equivalent to $\sim 15$ kernels out of the $\sim 317690$ particles leached. This corresponds to a binomial distribution defect fraction of $\leq 7.3 \mathrm{E}-5$ at $95 \%$ confidence, which is above the specified limit of $\leq 2.0 \mathrm{E}-5$. Analysis of as-coated TRISO particles from the same batch (G73J-14-93072A) showed a similar defect fraction. Further analysis determined that the source of the defects was cracked TRISO coatings on a small fraction of otherwise normal particles. This damage is thought to have occurred at $\mathrm{B} \& \mathrm{~W}$ during removal of the particles from the coating furnace via a suction transfer system. Because the suspected root cause of this nonconformance was not related to the compacting and characterization activities at ORNL, the nonconformance report was issued by INL. A decision was made to not use these compacts for the AGR-2 irradiation test because of the higher than desired level of exposed uranium in the as-manufactured fuel. This disposition was documented on INL NCR-44791. However, because this fuel has been well characterized, it will be retained in storage at INL and ORNL and may be used for methods development or other analyses.

Particles from 20 compacts were analyzed for uranium dispersion, which is an indicator of a defective IPyC layer. Excessive permeability in the IPyC may result in chlorine intrusion during 
$\mathrm{SiC}$ deposition and subsequent uranium leaching out of the kernel and into the buffer during compact heat treatment. A large defect fraction was observed, 443 out of 63538 particles. This corresponds to a binomial distribution defect fraction of $\leq 7.6 \mathrm{E}-3$ at $95 \%$ confidence, which is well above the specified limit of $\leq 1.0 \mathrm{E}-4$. Analysis of as-coated TRISO particles from the same batch (G73J-14-93072A) showed a similar defect fraction. The cause of the uranium dispersion is not completely known, although it is thought to be due to possible abnormal porosity in the IPyC layers of some of the particles. Because the suspected root cause of this nonconformance was not related to the compacting and characterization activities at ORNL, the nonconformance report was issued by INL. A decision was made to not use these compacts for the AGR-2 irradiation test because of the higher than desired level of uranium dispersion in the asmanufactured fuel. This disposition was documented on INL NCR-44792. However, because this fuel has been well characterized, it will be retained in storage at INL and ORNL and may be used for methods development or other analyses. 
Inspection Report Form IRF-12A: AGR-2 UCO Fuel Compact Lots

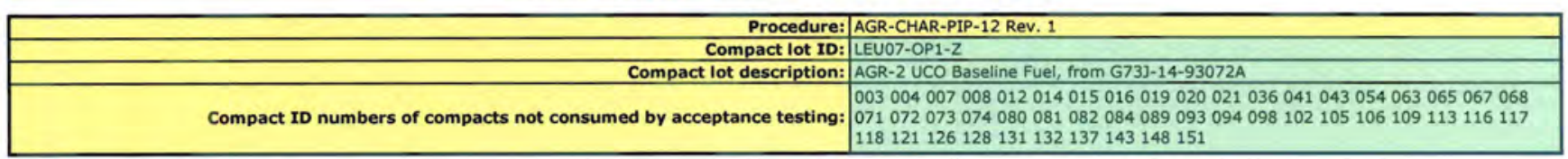

\begin{tabular}{|c|c|c|c|c|c|c|c|c|c|}
\hline \multirow[b]{2}{*}{ Property } & \multicolumn{4}{|c|}{ Measured Data } & \multirow{2}{*}{$\begin{array}{l}\text { Specification } \\
\text { INL SPC-923 } \\
\text { Revison } 3 \\
\end{array}$} & \multirow[b]{2}{*}{ Acceptance Criteria } & \multirow{2}{*}{$\begin{array}{l}\text { Acceptance } \\
\text { Test Value }\end{array}$} & \multirow{2}{*}{$\begin{array}{c}\text { Pass } \\
\text { or } \\
\text { fail }\end{array}$} & \multirow{2}{*}{$\begin{array}{c}\text { Data } \\
\text { Records }\end{array}$} \\
\hline & $\begin{array}{c}\text { Mean } \\
(x)\end{array}$ & \begin{tabular}{|c|} 
Std. Dev. \\
(s)
\end{tabular} & \begin{tabular}{|c|} 
Measurements \\
$(\mathrm{n})$
\end{tabular} & $\begin{array}{l}k \text { or } t \\
\text { value }\end{array}$ & & & & & \\
\hline \multirow{2}{*}{$\begin{array}{l}\text { Uranium loading } \\
\text { (gU/compact) }\end{array}$} & \multirow{2}{*}{1.251} & \multirow{2}{*}{0.005} & \multirow{2}{*}{6} & \multirow{2}{*}{2.015} & \multirow{2}{*}{$1.265 \pm 0.07$} & $A=x-t s / \sqrt{ } n \geq 1.195$ & 1.247 & pass & \multirow{2}{*}{ DRF-25 } \\
\hline & & & & & & $B=x+t s / \sqrt{n} \leq 1.335$ & 1.255 & pass & \\
\hline Compact diameter $(\mathrm{mm})$ & \multirow{3}{*}{\multicolumn{2}{|c|}{ See DRF-24 }} & & & $12.22-12.46$ & \multirow{3}{*}{$\begin{array}{l}\text { All available for irradiation test } \\
\text { meet specification }\end{array}$} & \multirow{3}{*}{ See DRF-24 } & fail & \multirow{3}{*}{ DRF-24 } \\
\hline Compact length (mm) & & & & & $25.02-25.40$ & & & 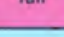 & \\
\hline Compact matrix density $\left(\mathrm{g} / \mathrm{cm}^{3}\right)$ & & & & & $\geq 1.45$ & & & pass & \\
\hline \multirow{2}{*}{$\begin{array}{l}\text { Iron content outside SiC } \\
(\mu g / \text { compact })\end{array}$} & \multirow{2}{*}{4.78} & \multirow{2}{*}{2.17} & \multirow{2}{*}{12} & 1.796 & $\begin{array}{l}\text { mean } \\
\leq 25\end{array}$ & $B=x+t s / \sqrt{n} \leq 25$ & 5.9 & pass & \multirow{2}{*}{$\begin{array}{l}\text { IRF-12B } \\
\text { DRF-26 }\end{array}$} \\
\hline & & & & 3.747 & $\begin{array}{c}\text { dispersion } \\
\leq 0.01 \geq 100 \\
\end{array}$ & $D=x+\sqrt{3} k s<100$ & 18.9 & pass & \\
\hline $\begin{array}{c}\text { Chromium content outside SiC } \\
\text { ( } \mu \mathrm{g} / \text { compact) }\end{array}$ & 0.59 & 0.06 & 12 & 1.796 & $\begin{array}{c}\text { mean } \\
\leq 50\end{array}$ & $B=x+t s / \sqrt{n} \leq 50$ & 0.6 & pass & $\begin{array}{l}\text { IRF-12B } \\
\text { DRF-26 } \\
\end{array}$ \\
\hline $\begin{array}{c}\text { Manganese content outside SiC } \\
(\mu \mathrm{g} / \text { compact })\end{array}$ & 0.20 & 0.09 & 12 & 1.796 & $\begin{array}{l}\text { mean } \\
\leq 50\end{array}$ & $B=x+t s / \sqrt{ } n \leq 50$ & 0.2 & pass & $\begin{array}{l}\text { IRF-12B } \\
\text { DRF-26 } \\
\end{array}$ \\
\hline $\begin{array}{c}\text { Cobalt content outside SiC } \\
\text { ( } \mu \mathrm{g} / \text { compact })\end{array}$ & 0.16 & 0.07 & 12 & 1.796 & $\begin{array}{l}\text { mean } \\
\leq 50\end{array}$ & $B=x+t s / \sqrt{n} \leq 50$ & 0.2 & pass & $\begin{array}{l}\text { IRF-12B } \\
\text { DRF-26 } \\
\end{array}$ \\
\hline $\begin{array}{c}\text { Nickel content outside SiC } \\
\text { ( } \mu \mathrm{g} / \text { compact) }\end{array}$ & 0.84 & 0.36 & 12 & 1.796 & $\begin{array}{c}\text { mean } \\
\leq 50\end{array}$ & $B=x+t s / \sqrt{ } n \leq 50$ & 1.0 & pass & $\begin{array}{l}\text { IRF-12B } \\
\text { DRF-26 } \\
\end{array}$ \\
\hline $\begin{array}{c}\mathrm{Cr}+\mathrm{Mn}+\mathrm{Co}+\mathrm{Ni} \text { content outside } \mathrm{SiC} \\
(\mu \mathrm{g} / \text { compact })\end{array}$ & 1.78 & 0.50 & 12 & 3.747 & $\begin{array}{c}\text { dispersion } \\
\leq 0.01 \geq 200 \\
\end{array}$ & $D=x+\sqrt{3} k s<200$ & 5.0 & pass & $\begin{array}{l}\text { IRF-12B } \\
\text { DRF-26 } \\
\end{array}$ \\
\hline $\begin{array}{c}\text { Calcium content outside SiC } \\
\text { ( } \mu \mathrm{g} / \text { compact) }\end{array}$ & 36.05 & 6.22 & 12 & 1.796 & $\begin{array}{l}\text { mean } \\
\leq 50\end{array}$ & $B=x+t s / \sqrt{n} \leq 50$ & 39.3 & pass & $\begin{array}{l}\text { IRF-12B } \\
\text { DRF-26 }\end{array}$ \\
\hline $\begin{array}{c}\text { Aluminum content outside SiC } \\
(\mu \mathrm{g} / \text { compact })\end{array}$ & 30.08 & 9.54 & 12 & 1.796 & $\begin{array}{l}\text { mean } \\
\leq 50 \\
\end{array}$ & $B=x+t s / \sqrt{n} \leq 50$ & 35.0 & pass & $\begin{array}{l}\text { IRF-12B } \\
\text { DRF-26 } \\
\end{array}$ \\
\hline $\begin{array}{c}\mathrm{Ti}+\mathrm{V} \text { content outside SiC } \\
(\mu \mathrm{g} / \text { compact })\end{array}$ & 21.28 & 1.04 & 12 & 1.796 & $\begin{array}{l}\text { mean } \\
\leq 240\end{array}$ & $B=x+t s / \sqrt{n} \leq 240$ & 21.8 & pass & $\begin{array}{l}\text { IRF-12B } \\
\text { DRF-26 }\end{array}$ \\
\hline
\end{tabular}

\begin{tabular}{|c|c|c|c|c|c|c|c|}
\hline \multirow[b]{2}{*}{ Property } & \multicolumn{2}{|c|}{ Measured Data } & \multirow{2}{*}{$\begin{array}{c}\text { Specification } \\
\text { INL SPC-923 } \\
\text { Revison } 2 \\
\end{array}$} & \multirow[b]{2}{*}{ Acceptance Criteria } & \multirow[b]{2}{*}{$\begin{array}{l}\text { Acceptance } \\
\text { Test Value }\end{array}$} & \multirow{2}{*}{$\begin{array}{c}\text { Pass } \\
\text { or } \\
\text { fail } \\
\end{array}$} & \multirow[b]{2}{*}{$\begin{array}{c}\text { Data } \\
\text { Records }\end{array}$} \\
\hline & $\begin{array}{c}\text { \# of } \\
\text { compacts }\end{array}$ & $\begin{array}{c}\text { \# of } \\
\text { particles }\end{array}$ & & & & & \\
\hline $\begin{array}{l}\text { Uranium contamination fraction } \\
\text { ( } g \text { exposed } U / \text { gram } U \text { in compact) }\end{array}$ & 100 & 317690 & $\leq 2.0 \times 10^{-5}$ & $\begin{array}{c}\leq 1 \text { effectively exposed kernel in } \geq 237192 \text { particles } \\
\text { or } \leq 2 \text { effectively exposed kernels in } \geq 314788 \text { particles } \\
\end{array}$ & 14.6 & fail & $\begin{array}{l}\text { IRF-12C } \\
\text { DRF-26 } \\
\end{array}$ \\
\hline $\begin{array}{l}\text { Defective SiC coating fraction } \\
\text { (fraction of total particles) }\end{array}$ & 60 & 190614 & $\leq 1.0 \times 10^{-4}$ & $\begin{array}{c}\leq 2 \text { leached kernels in } \geq 62956 \text { particles } \\
\text { or } \leq 6 \text { leached kernels in } \geq 118422 \text { particles }\end{array}$ & 0 & pass & $\begin{array}{l}\text { IRF-12D } \\
\text { DRF-26 }\end{array}$ \\
\hline $\begin{array}{l}\text { Defective IPyC coating fraction } \\
\text { (fraction of total particles) }\end{array}$ & 20 & 63538 & $\leq 1.0 \times 10^{-4}$ & $\begin{array}{l}\leq 1 \text { with excessive } U \text { dispersion in } \geq 47437 \text { particles } \\
\text { or } \leq 2 \text { with excessive } U \text { dispersion in } \geq 62956 \text { particles }\end{array}$ & 443 & fall & DRF-28 \\
\hline $\begin{array}{l}\text { Defective OPyC coating fraction } \\
\text { (fraction of total particles) }\end{array}$ & 1 & 3177 & $\leq 0.01$ & $\leq 6$ cracked or missing OPyC in $\geq 1182$ particles & 0 & pass & DRF-27 \\
\hline
\end{tabular}

\section{Comments}

One compact was outside of the length specification by $+0.046 \mathrm{~mm}$. This non-conformance was documented on ORNL NCR-X-MSTD-AGR-10-01 with a disposition of use as is.

A $15 / 317690$ uranium contamination fraction is above the specified limit and corresponds to $<7.3 \mathrm{e}-5$ at $95 \%$ confidence. This non-conformance was documented on INL NCR-44791 with a disposition of do not use for AGR-2 irradiation test, but retain for other uses.

A $443 / 63538$ defective IPYC coating fraction is above the specified limit and corresponds to $<7.6 \mathrm{e}-3$ at $95 \%$ confidence. This non-conformance was documented on INL NCR-44792 with a

disposition of do not use for AGR-2 irradiation test, but retain for other uses.
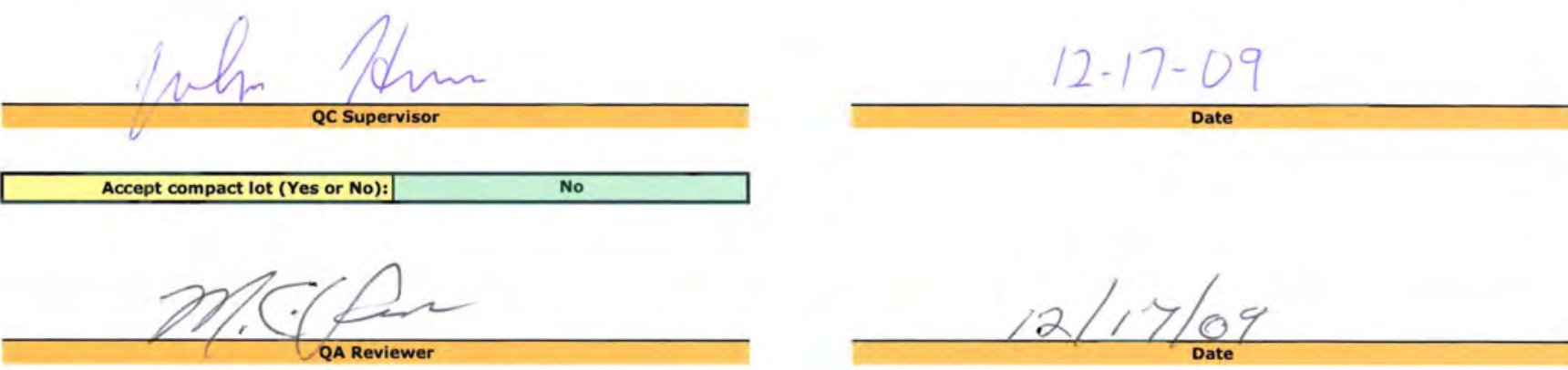
Inspection Report Form IRF-12B: Summary of Impurites Outside SiC - Maximum Corrected Values

\begin{tabular}{|r|l|}
\hline Procedure: & AGR-CHAR-DAM-26 Rev. 1 \\
\hline Operator: & Fred Montgomery \\
\hline Compact lot ID: & LEU07-OP1-Z \\
\hline Compact Lot description: & AGR-2 UCO Baseline Fuel, from G73J-14-93072A \\
\hline
\end{tabular}

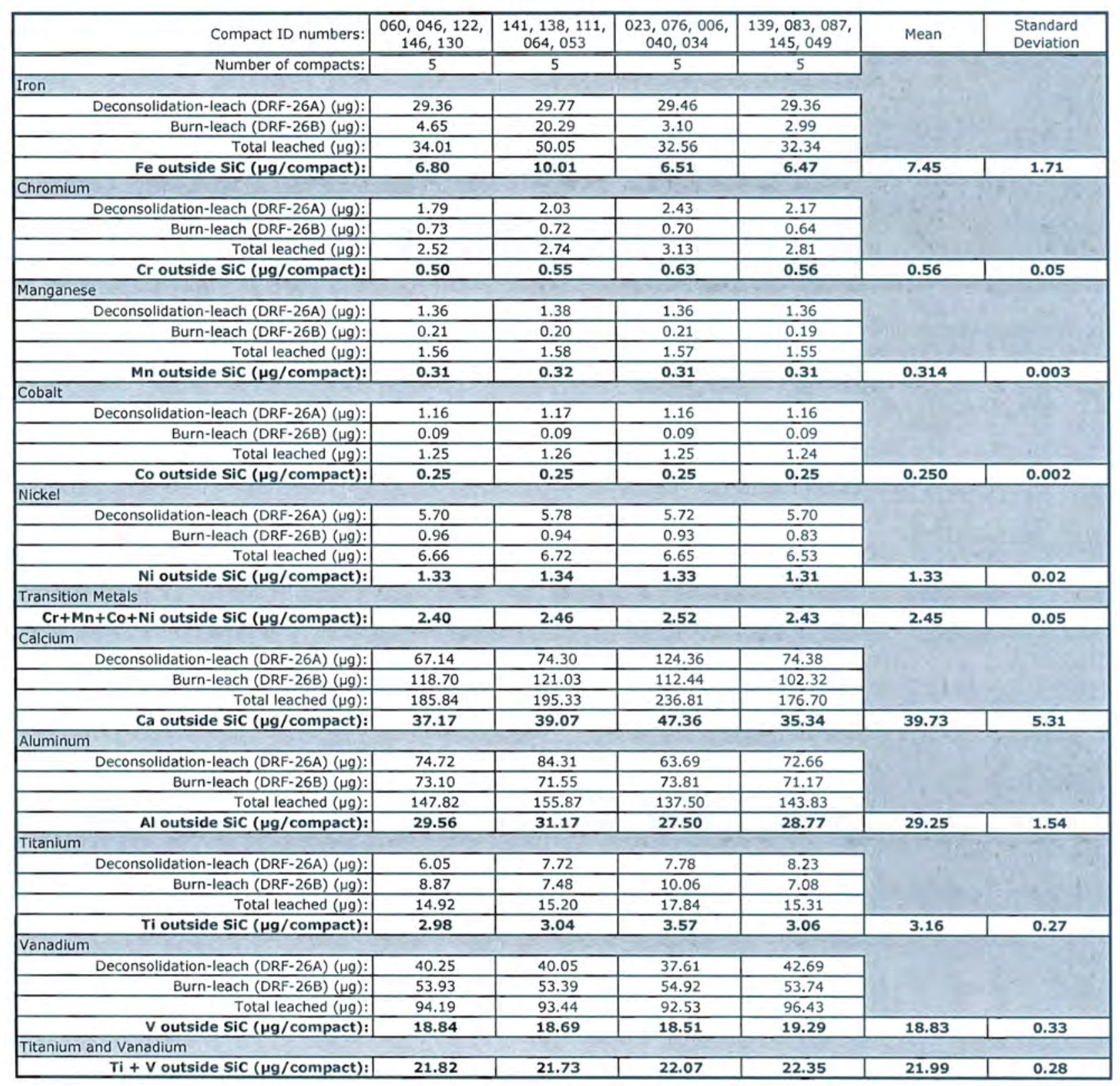

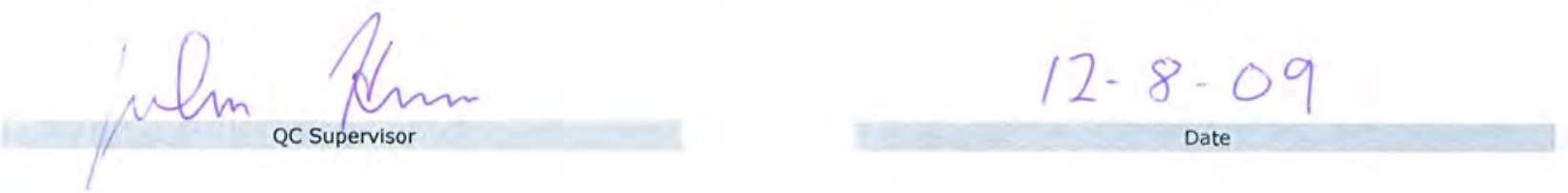


Inspection Report Form IRF-12B: Summary of Impurites Outside SiC - Maximum Corrected Values

\begin{tabular}{|r|l|}
\hline Procedure: & AGR-CHAR-DAM-26 Rev. 1 \\
\hline Operator: & Fred Montgomery \\
\hline Compact lot ID: & LEU07-OP1-Z \\
\hline Compact Lot description: & AGR-2 UCO Baseline Fuel, from G73J-14-93072A \\
\hline
\end{tabular}

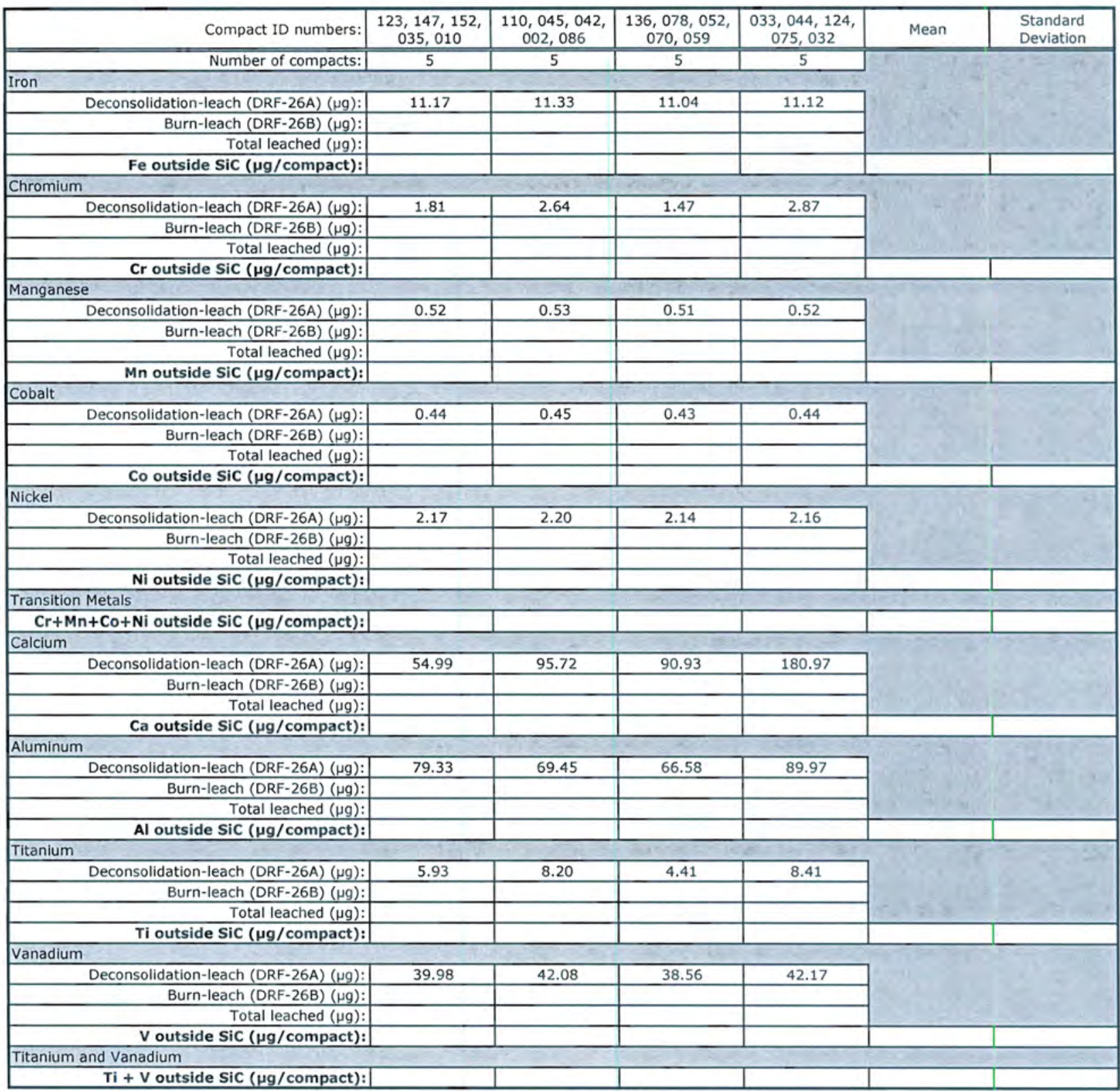

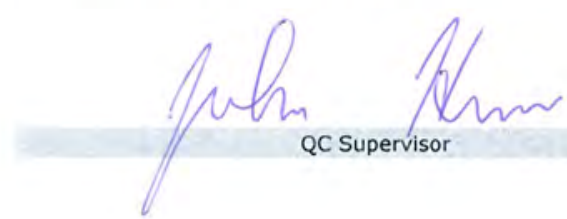

$$
12-8-09
$$


Inspection Report Form IRF-12B: Summary of Impurites Outside SiC - Maximum Corrected Values

\begin{tabular}{|r|l|}
\hline Procedure: & AGR-CHAR-PIP-12 Rev. 1 \\
\hline Operator: & Fred Montgomery \\
\hline Compact lot ID: & LEU07-OP1-Z \\
\hline Compact Lot description: & AGR-2 UCO Baseline Fuel, from G73J-14-93072A \\
\hline
\end{tabular}

\begin{tabular}{|c|c|c|c|c|c|c|}
\hline Compact ID numbers: & $\begin{array}{c}005,013,107 \\
058,062\end{array}$ & $\begin{array}{c}135,017,022 \\
018,125\end{array}$ & $\begin{array}{c}037,027,097 \\
025,048\end{array}$ & $\begin{array}{c}129,149,100, \\
029,066\end{array}$ & Mean & $\begin{array}{l}\text { Standard } \\
\text { Deviation }\end{array}$ \\
\hline Number of compacts: & 5 & 5 & 5 & 5 & & \\
\hline \multicolumn{7}{|l|}{ Iron } \\
\hline Deconsolidation-leach (DRF-26A) $(\mu g)$ : & 10.14 & 10.34 & 10.63 & 10.51 & & r \\
\hline Burn-leach (DRF-26B) $(\mu \mathrm{g})$ : & 5.67 & 9.44 & 6.63 & 8.28 & & \\
\hline Total leached $(\mu \mathrm{g})$ : & 15.80 & 19.78 & 17.26 & 18.79 & - & ( \\
\hline Fe outside SiC ( $\mu \mathrm{g} /$ compact): & 3.16 & 3.96 & 3.45 & 3.76 & 3.58 & 0.35 \\
\hline \multicolumn{7}{|l|}{ Chromium } \\
\hline Deconsolidation-leach (DRF-26A) $(\mu \mathrm{g})$ : & 2.21 & 2.71 & 1.90 & 2.47 & & \\
\hline Burn-leach (DRF-26B) $(\mu g)$ : & 0.76 & 0.89 & 0.89 & 0.90 & & \\
\hline Total leached $(\mu \mathrm{g}):$ & 2.97 & 3.60 & 2.79 & 3.38 & & \\
\hline Cr outside SiC ( $\mu \mathrm{g} /$ compact): & 0.59 & 0.72 & 0.56 & 0.68 & 0.64 & 0.07 \\
\hline \multicolumn{7}{|l|}{ Manganese } \\
\hline Deconsolidation-leach (DRF-26A) $(\mu \mathrm{g})$ : & 0.47 & 0.48 & 0.49 & 0.49 & & \\
\hline Burn-leach (DRF-26B) $(\mu \mathrm{g}):$ & 0.19 & 0.19 & 0.20 & 0.19 & & \\
\hline Total leached $(\mu \mathrm{g}):$ & 0.66 & 0.67 & 0.69 & 0.68 & & \\
\hline Mn outside SiC ( $\mu \mathrm{g} / \mathrm{compact}):$ & 0.13 & 0.13 & 0.14 & 0.14 & 0.135 & 0.002 \\
\hline \multicolumn{7}{|l|}{ ( } \\
\hline Deconsolidation-leach (DRF-26A) $(\mu \mathrm{g})$ : & 0.40 & 0.41 & 0.42 & 0.41 & & ( \\
\hline Burn-leach (DRF-26B) $(\mu \mathrm{g}):$ & 0.16 & 0.16 & 0.17 & 0.16 & & \\
\hline Total leached $(\mu \mathrm{g}):$ & 0.56 & 0.57 & 0.58 & 0.58 & & \\
\hline Co outside SiC ( $\mu \mathrm{g} /$ compact): & 0.11 & 0.11 & 0.12 & 0.12 & 0.115 & 0.002 \\
\hline \multicolumn{7}{|l|}{ Nickel } \\
\hline Deconsolidation-leach (DRF-26A) $(\mu g)$ : & 1.97 & 2.01 & 2.06 & 2.04 & & \\
\hline Burn-leach (DRF-26B) $(\mu \mathrm{g})$ : & 0.82 & 0.93 & 0.89 & 0.94 & & \\
\hline Total leached $(\mu \mathrm{g}):$ & 2.78 & 2.94 & 2.95 & 2.98 & & 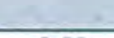 \\
\hline Ni outside SiC ( $\mu \mathrm{g} / \mathrm{compact}):$ & 0.56 & 0.59 & 0.59 & 0.60 & 0.58 & 0.02 \\
\hline \multicolumn{7}{|l|}{ Transition Metals } \\
\hline $\mathrm{Cr}+\mathrm{Mn}+\mathrm{Co}+\mathrm{Ni}$ outside SiC ( $\mu \mathrm{g} / \mathrm{compact}):$ & 1.39 & 1.55 & 1.40 & 1.52 & 1.47 & 0.08 \\
\hline \multicolumn{7}{|l|}{ Calcium } \\
\hline Deconsolidation-leach (DRF-26A) $(\mu \mathrm{g})$ : & 24.60 & 37.31 & 25.80 & 25.50 & & s \\
\hline Burn-leach (DRF-26B) $(\mu g)$ : & 136.72 & 160.04 & 167.65 & 199.10 & & : \\
\hline Total leached $(\mu g)$ : & 161.32 & 197.35 & 193.45 & 224.60 & & 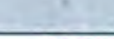 \\
\hline Ca outside SiC ( $\mu \mathrm{g} /$ compact): & 32.26 & 39.47 & 38.69 & 44.92 & 38.84 & 5.18 \\
\hline \multicolumn{7}{|l|}{ Aluminum } \\
\hline Deconsolidation-leach (DRF-26A) $(\mu \mathrm{g})$ : & 235.12 & 75.87 & 63.69 & 71.66 & & \\
\hline Burn-leach (DRF-26B) $(\mu g)$ : & 63.01 & 66.18 & 65.94 & 67.21 & & \\
\hline Total leached $(\mu \mathrm{g}):$ & 298.13 & 142.05 & 129.63 & 138.87 & & \\
\hline Al outside SiC ( $\mu \mathrm{g} / \mathrm{compact})$ : & 59.63 & 28.41 & 25.93 & 27.77 & 35.43 & 16.16 \\
\hline \multicolumn{7}{|l|}{ Titanium } \\
\hline Deconsolidation-leach (DRF-26A) $(\mu \mathrm{g})$ : & 6.75 & 9.00 & 6.76 & 9.90 & & \\
\hline Burn-leach (DRF-26B) $(\mu g)$ : & 7.42 & 5.70 & 8.12 & 5.82 & & \\
\hline Total leached $(\mu \mathrm{g}):$ & 14.17 & 14.70 & 14.88 & 15.72 & & \\
\hline Ti outside SiC ( $\mu \mathrm{g} /$ compact): & 2.83 & 2.94 & 2.98 & 3.14 & 2.97 & 0.13 \\
\hline \multicolumn{7}{|l|}{ Vanadium } \\
\hline Deconsolidation-leach (DRF-26A) $(\mu \mathrm{g})$ : & 38.05 & 44.71 & 37.05 & 41.40 & & \\
\hline Burn-leach (DRF-26B) $(\mu g)$ : & 48.96 & 50.11 & 46.19 & 46.00 & & \\
\hline Total leached $(\mu \mathrm{g}):$ & 87.01 & 94.82 & 83.24 & 87.40 & & 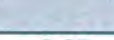 \\
\hline V outside SiC $(\mu \mathrm{g} /$ compact $):$ & 17.40 & 18.96 & 16.65 & 17.48 & 17.62 & 0.97 \\
\hline \multicolumn{7}{|l|}{ Titanium and Vanadium } \\
\hline $\mathrm{Ti}+\mathrm{V}$ outside SiC $(\mu \mathrm{g} /$ compact $):$ & 20.24 & 21.90 & 19.62 & 20.62 & 20.60 & 0.96 \\
\hline
\end{tabular}

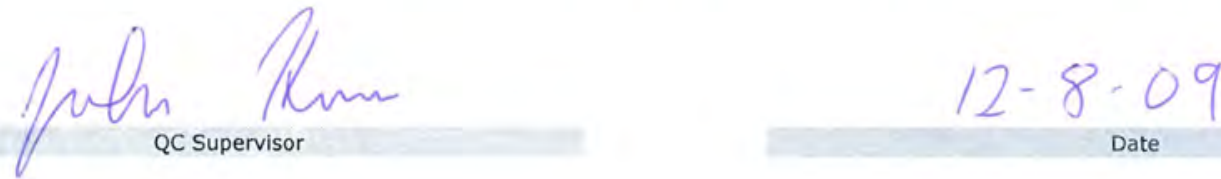


Inspection Report Form IRF-12B: Summary of Impurites Outside SiC - Maximum Corrected Values

\begin{tabular}{|r|l|}
\hline Procedure: & AGR-CHAR-PIP-12 Rev. 1 \\
\hline Operator: & Fred Montgomery \\
\hline Compact lot ID: & LEU07-OP1-Z \\
\hline Compact Lot description: & AGR-2 UCO Baseline Fuel, from G73J-14-93072A \\
\hline
\end{tabular}

\begin{tabular}{|c|c|c|c|c|c|c|}
\hline Compact ID numbers: & $\begin{array}{c}055,030,085 \\
108,140\end{array}$ & \begin{tabular}{|c|}
$026,103,095$ \\
119,077 \\
\end{tabular} & $\begin{array}{c}112,009,155 \\
056,001\end{array}$ & $\begin{array}{c}144,134,051 \\
115,088\end{array}$ & Mean & $\begin{array}{l}\text { Standard } \\
\text { Deviation }\end{array}$ \\
\hline Number of compacts: & 5 & 5 & 5 & 5 & & \\
\hline \multicolumn{5}{|l|}{ Iron } & & \\
\hline Deconsolidation-leach (DRF-26A) $(\mu \mathrm{g})$ : & 10.44 & 10.79 & 10.67 & 10.51 & & \\
\hline Burn-leach (DRF-26B) $(\mu g)$ : & 5.65 & 5.97 & 6.63 & 5.76 & & \\
\hline Total leached $(\mu \mathrm{g}):$ & 16.09 & 16.76 & 17.30 & 16.26 & & \\
\hline Fe outside SiC ( $\mu \mathrm{g} /$ compact $):$ & 3.22 & 3.35 & 3.46 & 3.25 & 3.32 & 0.11 \\
\hline \multicolumn{5}{|l|}{ Chromium } & & \\
\hline Deconsolidation-leach (DRF-26A) $(\mu \mathrm{g})$ : & 2.33 & 2.11 & 1.86 & 1.90 & & \\
\hline Burn-leach (DRF-26B) $(\mu g)$ : & 0.72 & 0.78 & 0.86 & 0.70 & & \\
\hline Total leached $(\mu \mathrm{g}):$ & 3.04 & 2.89 & 2.72 & 2.60 & & \\
\hline Cr outside SiC ( $\mu g /$ compact): & 0.61 & 0.58 & 0.54 & 0.52 & 0.56 & 0.04 \\
\hline \multicolumn{5}{|l|}{ Manganese } & \multirow{4}{*}{ 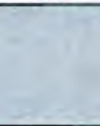 } & \multirow[b]{4}{*}{-} \\
\hline Deconsolidation-leach (DRF-26A) $(\mu g)$ : & 0.49 & 0.50 & 0.49 & 0.49 & & \\
\hline Burn-leach (DRF-26B) $(\mu g):$ & 0.19 & 0.19 & 0.20 & 0.19 & & \\
\hline Total leached $(\mu \mathrm{g}):$ & 0.68 & 0.69 & 0.69 & 0.68 & & \\
\hline Mn outside SiC ( $\mu \mathrm{g} /$ compact): & 0.14 & 0.14 & 0.14 & 0.14 & \multirow{5}{*}{0.137} & 0.001 \\
\hline \multicolumn{5}{|l|}{ Cobalt } & & \multirow{5}{*}{0.001} \\
\hline \multirow{4}{*}{$\begin{array}{r}\text { Total leached }(\mu \mathrm{g}): \\
\text { Co outside SiC }(\mu \mathrm{g} / \text { compact }):\end{array}$} & 0.41 & 0.42 & 0.42 & 0.41 & & \\
\hline & 0.16 & 0.16 & 0.17 & 0.16 & & \\
\hline & 0.57 & 0.59 & 0.59 & 0.58 & & \\
\hline & 0.11 & 0.12 & 0.12 & 0.12 & 0.116 & \\
\hline \multicolumn{5}{|l|}{ 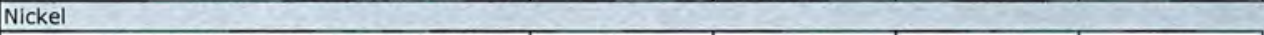 } & \multirow[t]{4}{*}{+2} & \\
\hline Deconsolidation-leach (DRF-26A) $(\mu \mathrm{g})$ : & 2.02 & 2.10 & 2.07 & 2.04 & & \\
\hline Burn-leach (DRF-26B) $(\mu \mathrm{g})$ : & 1.11 & 0.93 & 0.90 & 0.85 & & \\
\hline Total leached $(\mu \mathrm{g})$ : & 3.13 & 3.02 & 2.97 & 2.89 & & n \\
\hline Ni outside SiC $(\mu \mathrm{g} /$ compact $):$ & 0.63 & 0.60 & 0.59 & 0.58 & 0.60 & 0.02 \\
\hline \multicolumn{7}{|l|}{ Transition Metals } \\
\hline $\mathrm{Cr}+\mathrm{Mn}+\mathrm{Co}+\mathrm{Ni}$ outside $\mathrm{SiC}(\mu \mathrm{g} /$ compact $):$ & 1.49 & 1.44 & 1.39 & 1.35 & 1.42 & 0.06 \\
\hline \multicolumn{5}{|l|}{ Calcium } & & \\
\hline Deconsolidation-leach (DRF-26A) $(\mu \mathrm{g})$ : & 45.30 & 39.18 & 49.14 & 25.50 & & \\
\hline Burn-leach (DRF-26B) $(\mu \mathrm{g}):$ & 109.74 & 116.39 & 90.73 & 115.81 & & $4 x^{4}$ \\
\hline Total leached $(\mu \mathrm{g}):$ & 155.04 & 155.57 & 139.87 & 141.31 & & 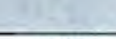 \\
\hline Ca outside SiC ( $\mu \mathrm{g} /$ compact): & 31.01 & 31.11 & 27.97 & 28.26 & 29.59 & 1.70 \\
\hline Aluminum & & & & & & \\
\hline Deconsolidation-leach (DRF-26A) $(\mu \mathrm{g})$ : & 71.17 & 64.86 & 56.07 & 63.95 & & \\
\hline Burn-leach (DRF-26B) $(\mu \mathrm{g})$ : & 60.71 & 74.56 & 62.78 & 57.00 & & \\
\hline Total leached $(\mu \mathrm{g}):$ & 131.89 & 139.42 & 118.85 & 120.96 & & \\
\hline Al outside SiC $(\mu \mathrm{g} /$ compact $):$ & 26.38 & 27.88 & 23.77 & 24.19 & 25.56 & 1.93 \\
\hline Titanium & & & & & & \\
\hline Deconsolidation-leach (DRF-26A) $(\mu \mathrm{g})$ : & 7.65 & 7.17 & 5.31 & 6.62 & 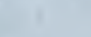 & \\
\hline Burn-leach (DRF-26B) $(\mu \mathrm{g})$ : & 7.95 & 8.20 & 9.06 & 8.23 & & \\
\hline Total leached $(\mu \mathrm{g}):$ & 15.60 & 15.37 & 14.37 & 14.85 & & \\
\hline Ti outside SiC ( $\mu \mathrm{g} / \mathrm{compact}):$ & 3.12 & 3.07 & 2.87 & 2.97 & 3.01 & 0.11 \\
\hline Vanadium & & & & & & \\
\hline Deconsolidation-leach (DRF-26A) $(\mu g)$ : & 42.77 & 42.07 & 39.93 & 38.82 & & \\
\hline Burn-leach (DRF-26B) $(\mu \mathrm{g})$ : & 49.25 & 55.51 & 52.53 & 43.79 & & \\
\hline Total leached $(\mu \mathrm{g}):$ & 92.02 & 97.58 & 92.46 & 82.61 & & \\
\hline V outside SiC ( $\mu \mathrm{g} /$ compact): & 18.40 & 19.52 & 18.49 & 16.52 & 18.23 & 1.25 \\
\hline Titanium and Vanadium & & & & 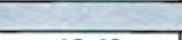 & & \\
\hline $\mathrm{Ti}+\mathrm{V}$ outside $\mathrm{SiC}(\mu \mathrm{g} /$ compact $):$ & 21.52 & 22.59 & 21.37 & 19.49 & 21.24 & 1.29 \\
\hline
\end{tabular}

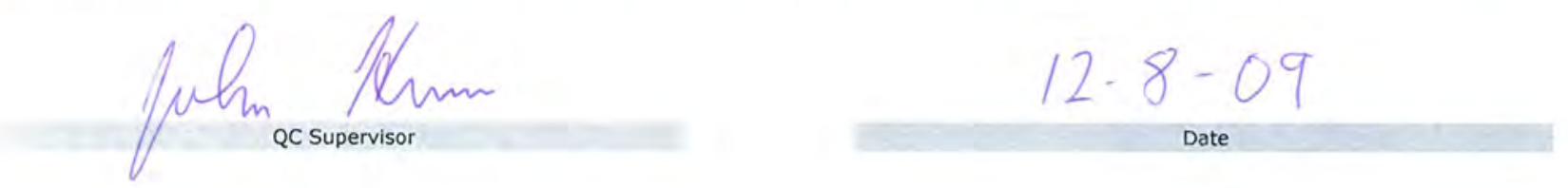


Inspection Report Form IRF-12B: Summary of Impurites Outside SiC - Maximum Corrected Values

\begin{tabular}{|r|l|}
\hline Procedure: & AGR-CHAR-PIP-12 Rev. 1 \\
\hline Operator: & Fred Montgomery \\
\hline Compact lot ID: & LEU07-OP1-Z \\
\hline Compact Lot description: & AGR-2 UCO Baseline Fuel, from G73J-14-93072A \\
\hline
\end{tabular}

\begin{tabular}{|c|c|c|c|c|c|c|}
\hline Compact ID numbers: & $\begin{array}{c}142,047,011 \\
092,038\end{array}$ & $\begin{array}{c}069,050,039 \\
150,028 \\
\end{array}$ & $\begin{array}{c}114,133,127 \\
091,061 \\
\end{array}$ & $\begin{array}{c}099,024,031, \\
104,101\end{array}$ & Mean & $\begin{array}{l}\text { Standard } \\
\text { Deviation }\end{array}$ \\
\hline Number of compacts: & 5 & 5 & 5 & 5 & & \\
\hline \multicolumn{5}{|l|}{ Iron } & & \\
\hline Deconsolidation-leach (DRF-26A) $(\mu g):$ & 11.58 & 11.17 & 11.49 & 11.33 & & \\
\hline \multirow{2}{*}{\multicolumn{7}{|c|}{$\begin{array}{r}\text { Burn-leach (DRF-26B) }(\mu g): \\
\text { Total leached }(\mu g):\end{array}$}} \\
\hline & & & & & & \\
\hline \multicolumn{7}{|l|}{ Fe outside SiC $(\mu \mathrm{g} /$ compact $)$ : } \\
\hline \multicolumn{5}{|l|}{\begin{tabular}{|l|l} 
Chromium \\
\end{tabular}} & & \\
\hline Deconsolidation-leach (DRF-26A) $(\mu \mathrm{g})$ : & 1.53 & 0.99 & 1.20 & 1.16 & & \\
\hline \multirow{2}{*}{\multicolumn{7}{|c|}{$\begin{array}{r}\text { Burn-leach (DRF-26B) }(\mu g) \text { : } \\
\text { Total leached }(\mu g) \text { : }\end{array}$}} \\
\hline & & & & & & \\
\hline \multicolumn{7}{|l|}{ Cr outside SiC $(\mu \mathrm{g} /$ compact $):$} \\
\hline \multicolumn{7}{|l|}{ Manganese } \\
\hline Deconsolidation-leach (DRF-26A) $(\mu \mathrm{g}):$ & 0.54 & 0.52 & 0.53 & 0.53 & & \\
\hline \multicolumn{7}{|l|}{ Burn-leach (DRF-26B) $(\mu \mathrm{g}):$} \\
\hline \multicolumn{7}{|l|}{ Total leached $(\mu \mathrm{g}):$} \\
\hline \multicolumn{7}{|l|}{ Mn outside SiC $(\mu \mathrm{g} /$ compact $):$} \\
\hline \multicolumn{7}{|l|}{ 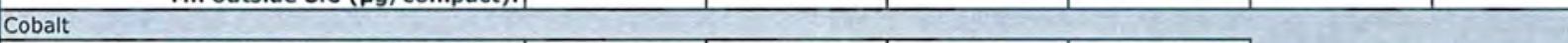 } \\
\hline Deconsolidation-leach (DRF-26A) $(\mu \mathrm{g})$ : & 0.46 & 0.44 & 0.45 & 0.45 & & \\
\hline \multicolumn{7}{|l|}{ Burn-leach (DRF-26B) $(\mu g):$} \\
\hline \multicolumn{7}{|l|}{ Total leached $(\mu \mathrm{g}):$} \\
\hline \multicolumn{7}{|l|}{ Co outside SiC $(\mu \mathrm{g} /$ compact): } \\
\hline \multicolumn{7}{|l|}{ Nickel } \\
\hline Deconsolidation-leach (DRF-26A) $(\mu \mathrm{g})$ : & 2.25 & 2.17 & 2.23 & 2.20 & & \\
\hline \multicolumn{7}{|l|}{ Burn-leach (DRF-26B) $(\mu \mathrm{g}):$} \\
\hline \multicolumn{7}{|l|}{ Total leached $(\mu \mathrm{g}):$} \\
\hline \multicolumn{7}{|l|}{ Ni outside SiC $(\mu \mathrm{g} /$ compact): } \\
\hline \multicolumn{7}{|l|}{ Transition Metals } \\
\hline \multicolumn{7}{|l|}{$\mathrm{Cr}+\mathrm{Mn}+\mathrm{Co}+\mathrm{Ni}$ outside $\mathrm{SiC}(\mu \mathrm{g} /$ compact $):$} \\
\hline Calcium & & & & 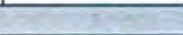 & & \\
\hline Deconsolidation-leach (DRF-26A) $(\mu \mathrm{g})$ : & 12.60 & 13.00 & 38.38 & 12.60 & & \\
\hline Burn-leach (DRF-26B) $(\mu \mathrm{g})$ : & & & & & & \\
\hline Total leached $(\mu \mathrm{g})$ : & & & & & & \\
\hline Ca outside SiC $(\mu \mathrm{g} /$ compact): & & & & & & \\
\hline Aluminum & & & & & & \\
\hline Deconsolidation-leach (DRF-26A) $(\mu \mathrm{g})$ : & 69.28 & 62.29 & 72.61 & 67.33 & & \\
\hline Burn-leach (DRF-26B) $(\mu \mathrm{g})$ : & & & & & & \\
\hline Total leached $(\mu \mathrm{g})$ : & & & & & & \\
\hline Al outside SiC $(\mu \mathrm{g} /$ compact): & & & & & & \\
\hline Titanium & & & & 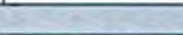 & & \\
\hline Deconsolidation-leach (DRF-26A) $(\mu \mathrm{g})$ : & 7.89 & 4.15 & 7.78 & 5.10 & & \\
\hline Burn-leach (DRF-26B) $(\mu \mathrm{g})$ : & & & & & & \\
\hline Total leached $(\mu \mathrm{g})$ : & & & & & & \\
\hline Ti outside SiC ( $\mu \mathrm{g} /$ compact): & & & & & & \\
\hline Vanadium & & & & & & \\
\hline Deconsolidation-leach (DRF-26A) $(\mu \mathrm{g})$ : & 38.23 & 36.52 & 39.57 & 41.64 & & \\
\hline Burn-leach (DRF-26B) $(\mu \mathrm{g})$ : & & & & & & \\
\hline Total leached $(\mu \mathrm{g})$ : & & & & & & \\
\hline V outside $\operatorname{SiC}(\mu \mathrm{g} /$ compact $)$ : & & & & & & \\
\hline Titanium and Vanadium & & & & & & \\
\hline $\mathrm{Ti}+\mathrm{V}$ outside $\mathrm{SiC}(\mu \mathrm{g} /$ compact $)$ & & & & & & \\
\hline
\end{tabular}
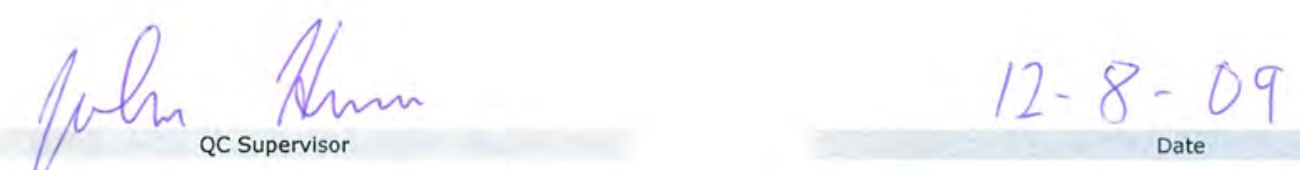


\section{Inspection Report Form IRF-12C: Summary of Uranium Contamination}

\begin{tabular}{|r|l|}
\hline Procedure: & AGR-CHAR-PIP-12 Rev. 1 \\
\hline Operator: & Fred Montgomery \\
\hline Compact lot ID: & LEU07-OP1-Z \\
\hline Compact Lot description: & AGR-2 UCO Baseline Fuel, from G73J-14-93072A \\
\hline
\end{tabular}

\begin{tabular}{|r|c|c|c|c|c|}
\hline Compact ID numbers: & $\begin{array}{c}060,046,122, \\
146,130\end{array}$ & $\begin{array}{c}141,138,111, \\
064,053\end{array}$ & $\begin{array}{c}023,076,006, \\
040,034\end{array}$ & $\begin{array}{c}139,083,087, \\
145,049\end{array}$ & Total \\
\hline \hline Number of compacts: & 5 & 5 & 5 & 5 & 20 \\
\hline Effective number of exposed kernels: & 1.1 & 1.1 & 0.0 & 0.6 & 2.8 \\
\hline
\end{tabular}

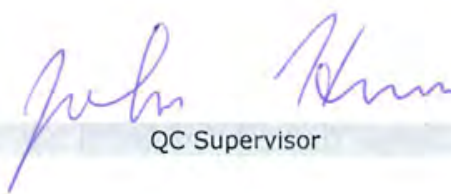

$$
12-8 \cdot 09
$$

Date 
Inspection Report Form IRF-12C: Summary of Uranium Contamination

\begin{tabular}{|r|l|}
\hline Procedure: & AGR-CHAR-PIP-12 Rev. 1 \\
\hline Operator: & Fred Montgomery \\
\hline Compact lot ID: & LEU07-OP1-Z \\
\hline Compact Lot description: & AGR-2 UCO Baseline Fuel, from G73J-14-93072A \\
\hline
\end{tabular}

\begin{tabular}{|r|c|c|c|c|c|}
\hline Compact ID numbers: & $\begin{array}{c}123,147,152, \\
035,010\end{array}$ & $\begin{array}{c}110,045,042, \\
002,086\end{array}$ & $\begin{array}{c}136,078,052, \\
070,059\end{array}$ & $\begin{array}{c}033,044,124, \\
075,032\end{array}$ & Total \\
\hline \hline Number of compacts: & 5 & 5 & 5 & 5 & 20 \\
\hline Effective number of exposed kernels: & 0.0 & 2.0 & 1.1 & 0.2 & 3.3 \\
\hline
\end{tabular}

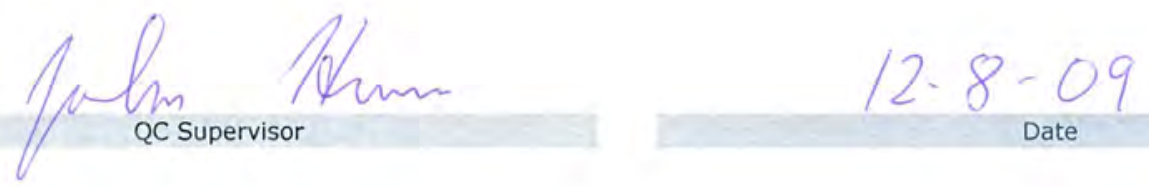


Inspection Report Form IRF-12C: Summary of Uranium Contamination

\begin{tabular}{|r|l|}
\hline Procedure: & AGR-CHAR-PIP-12 Rev. 1 \\
\hline Operator: & Fred Montgomery \\
\hline Compact lot ID: & LEU07-OP1-Z \\
\hline Compact Lot description: & AGR-2 UCO Baseline Fuel, from G73J-14-93072A \\
\hline
\end{tabular}

\begin{tabular}{|r|c|c|c|c|c|}
\hline Compact ID numbers: & $\begin{array}{c}005,013,107, \\
058,062\end{array}$ & $\begin{array}{c}135,017,022, \\
018,125\end{array}$ & $\begin{array}{c}037,027,097, \\
025,048\end{array}$ & $\begin{array}{c}129,149,100, \\
029,066\end{array}$ & Total \\
\hline \hline Number of compacts: & 5 & 5 & 5 & 5 & 20 \\
\hline Effective number of exposed kernels: & 2.0 & 0.0 & 0.0 & 1.0 & 3.1 \\
\hline
\end{tabular}

Pon $12-8-09$


Inspection Report Form IRF-12C: Summary of Uranium Contamination

Procedure: AGR-CHAR-PIP-12 Rev. 1

Operator: Fred Montgomery

Compact lot ID: LEU07-OP1-Z

Compact Lot description: AGR-2 UCO Baseline Fuel, from G73J-14-93072A

\begin{tabular}{|r|c|c|c|c|c|}
\hline Compact ID numbers: & $\begin{array}{c}055,030,085, \\
108,140\end{array}$ & $\begin{array}{c}026,103,095, \\
119,077\end{array}$ & $\begin{array}{c}112,009,155, \\
056,001\end{array}$ & $\begin{array}{c}144,134,051, \\
115,088\end{array}$ & Total \\
\hline \hline Number of compacts: & 5 & 5 & 5 & 5 & 20 \\
\hline Effective number of exposed kernels: & 1.1 & 1.1 & 0.0 & 0.0 & 2.2 \\
\hline
\end{tabular}

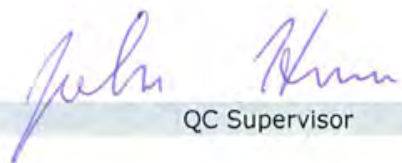

$$
\operatorname{12}_{\text {Date }}
$$


ORNL/TM-2009/305

Inspection Report Form IRF-12C: Summary of Uranium Contamination

Procedure: AGR-CHAR-PIP-12 Rev. 1

Operator: Fred Montgomery

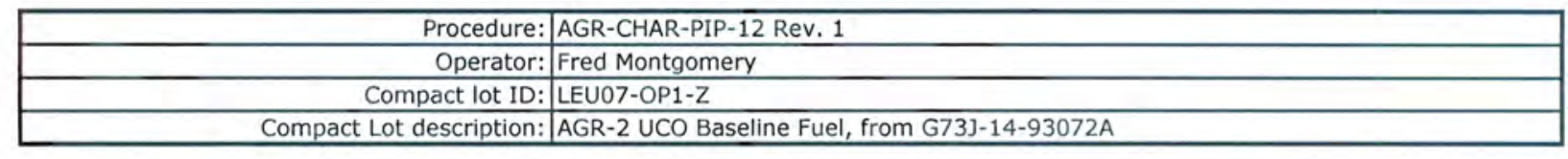

\begin{tabular}{|r|c|c|c|c|c|}
\hline Compact ID numbers: & $\begin{array}{c}142,047,011, \\
092,038\end{array}$ & $\begin{array}{c}069,050,039, \\
150,028\end{array}$ & $\begin{array}{c}114,133,127, \\
091,061\end{array}$ & $\begin{array}{c}099,024,031, \\
104,101\end{array}$ & Total \\
\hline \hline Number of compacts: & 5 & 5 & 5 & 5 & 20 \\
\hline Effective number of exposed kernels: & 0.0 & 0.0 & 2.3 & 1.0 & 3.3 \\
\hline
\end{tabular}

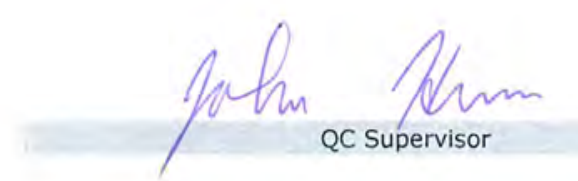

$$
\underset{\text { Date }}{12-8-09}
$$

54 
Inspection Report Form IRF-12D: Summary of Burn Leach Defects

\begin{tabular}{|r|l|}
\hline Procedure: & AGR-CHAR-PIP-12 Rev. 1 \\
\hline Operator: & Fred Montgomery \\
\hline Compact lot ID: & LEU07-OP1-Z \\
\hline Compact Lot description: & AGR-2 UCO Baseline Fuel, from G73J-14-93072A \\
\hline
\end{tabular}

\begin{tabular}{|r|c|c|c|c|c|}
\hline Compact ID numbers: & $\begin{array}{c}060,046,122, \\
146,130\end{array}$ & $\begin{array}{c}141,138,111, \\
064,053\end{array}$ & $\begin{array}{c}023,076,006, \\
040,034\end{array}$ & $\begin{array}{c}139,083,087, \\
145,049\end{array}$ & Total \\
\hline \hline Number of compacts: & 5 & 5 & 5 & 5 & 20 \\
\hline Number of leached kernels: & 0 & 0 & 0 & 0 & 0 \\
\hline
\end{tabular}

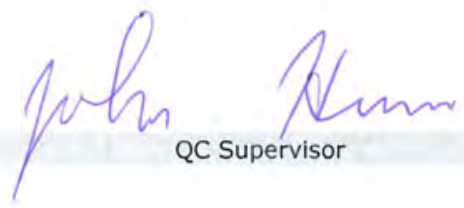

$$
12-8-09
$$


ORNL/TM-2009/305

Inspection Report Form IRF-12D: Summary of Burn Leach Defects

Procedure: AGR-CHAR-PIP-12 Rev. 1

Operator: Fred Montgomery

Compact lot ID: LEU 07-OP1-Z

Compact Lot description: AGR-2 UCO Baseline Fuel, from G73J-14-93072A

\begin{tabular}{|r|c|c|c|c|c|}
\hline Compact ID numbers: & $\begin{array}{c}005,013,107, \\
058,062\end{array}$ & $\begin{array}{c}135,017,022, \\
018,125\end{array}$ & $\begin{array}{c}037,027,097, \\
025,048\end{array}$ & $\begin{array}{c}129,149,100, \\
029,066\end{array}$ & Total \\
\hline \hline Number of compacts: & 5 & 5 & 5 & 5 & 20 \\
\hline Number of leached kernels: & 0 & 0 & 0 & 0 & 0 \\
\hline
\end{tabular}

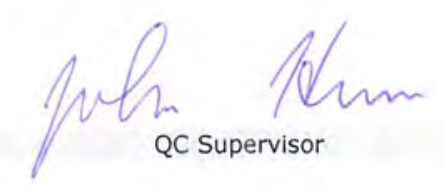

$12-8-09$

56 
ORNL/TM-2009/305

Inspection Report Form IRF-12D: Summary of Burn Leach Defects

Procedure: AGR-CHAR-PIP-12 Rev. 1

Operator: Fred Montgomery

$\begin{aligned} \text { Compact lot ID: } & \text { LEU 07-OP1-Z } \\ \text { Compact Lot description: } & \text { AGR -2 UCO Baseline Fuel, from G73J-14-93072A }\end{aligned}$

\begin{tabular}{|r|c|c|c|c|c|}
\hline Compact ID numbers: & $\begin{array}{c}055,030,085, \\
108,140\end{array}$ & $\begin{array}{c}026,103,095, \\
119,077\end{array}$ & $\begin{array}{c}112,009,155, \\
056,001\end{array}$ & $\begin{array}{c}144,134,051, \\
115,088\end{array}$ & Total \\
\hline \hline Number of compacts: & 5 & 5 & 5 & 5 & 20 \\
\hline Number of leached kernels: & 0 & 0 & 0 & 0 & 0 \\
\hline
\end{tabular}

Whim Qc Supervisor

$12.8-09$

57 
Data Report Form DRF-24A: Compact Dlameter and Length

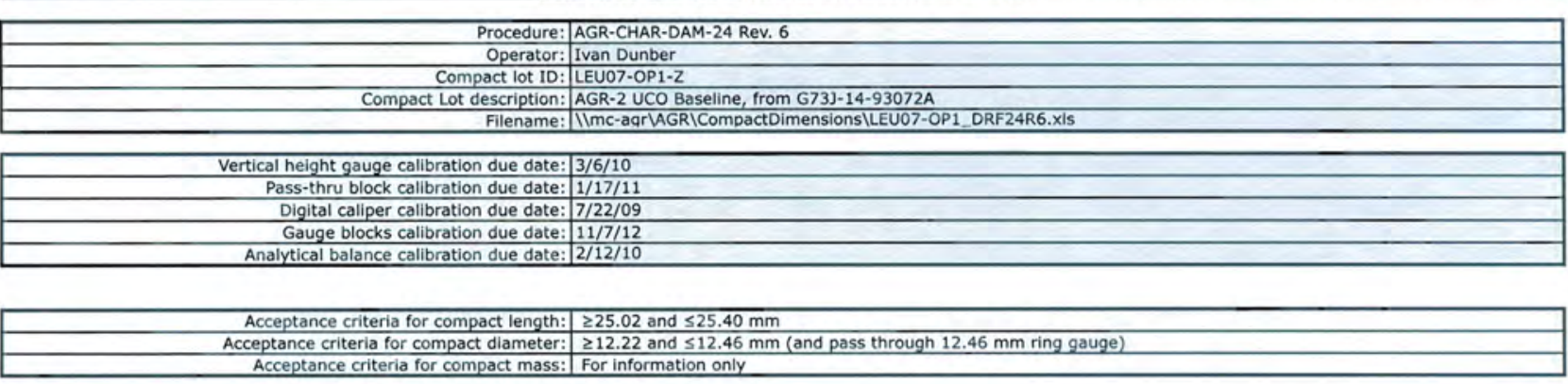

\begin{tabular}{|c|c|c|c|c|c|c|c|c|c|c|}
\hline \multirow{2}{*}{$\begin{array}{l}\text { Compact } \\
\text { ID Number } \\
\end{array}$} & \multirow{2}{*}{$\begin{array}{l}\text { Length } \\
(\mathrm{mm})\end{array}$} & \multicolumn{6}{|c|}{ Diameter $(\mathrm{mm})$} & \multirow{2}{*}{$\begin{array}{c}\text { Pass Thru? } \\
(Y \text { or } \mathrm{N})\end{array}$} & \multirow{2}{*}{$\begin{array}{l}\text { Compact weight } \\
\text { (a) }\end{array}$} & \multirow{2}{*}{$\begin{array}{c}\text { Accept? } \\
\text { (pass or fail) }\end{array}$} \\
\hline & & Top 1 & Top 2 & Middle 1 & Middle 2 & Bottom 1 & Bottom 2 & & & \\
\hline 2001 & 25.242 & 12.31 & 12.31 & 12.32 & 12.32 & 12.31 & 12.31 & Y & 6.3449 & pass \\
\hline 2002 & 25.284 & 12.31 & 12.31 & 12.32 & 12.32 & 12.31 & 12.31 & $Y$ & 6.3501 & pass \\
\hline 2003 & 25.159 & 12.31 & 12.31 & 12.32 & 12.32 & 12.32 & 12.32 & 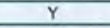 & 6.3504 & pass \\
\hline 2004 & 25.124 & 12.31 & 12.30 & 12.32 & 12.32 & 12.31 & 12.31 & Y & 6.3500 & pass \\
\hline 2005 & 25.218 & 12.31 & 12.31 & 12.32 & 12.32 & 12.31 & 12.31 & Y & 6.3466 & pass \\
\hline 2006 & 25.225 & 12.31 & 12.31 & 12.33 & 12.32 & 12.32 & 12.32 & Y & 6.3508 & pass \\
\hline 2007 & 25.242 & 12.31 & 12.31 & 12.32 & 12.32 & 12.31 & 12.32 & $\mathrm{Y}$ & 6.3453 & pass \\
\hline 2008 & 25.197 & 12.32 & 12.33 & 12.33 & 12.34 & 12.33 & 12.33 & $\mathrm{Y}$ & 6.3609 & pass \\
\hline 2009 & 25.165 & 12.30 & 12.31 & 12.32 & 12.32 & 12.31 & 12.31 & Y & 6.3542 & pass \\
\hline 2010 & 25.204 & 12.31 & 12.31 & 12.32 & 12.32 & 12.31 & 12.30 & Y & 6.3419 & pass \\
\hline$Z 011$ & 25.144 & 12.31 & 12.31 & 12.32 & 12.32 & 12.30 & 12.30 & $Y$ & 6.3376 & pass \\
\hline Z012 & 25.358 & 12.31 & 12.30 & 12.31 & 12.31 & 12.30 & 12.31 & Y & 6.3532 & pass \\
\hline 2013 & 25.217 & 12.30 & 12.30 & 12.32 & 12.32 & 12.31 & 12.31 & Y & 6.3393 & pass \\
\hline 2014 & 25.212 & 12.30 & 12.31 & 12.32 & 12.32 & 12.31 & 12.31 & Y & 6.3355 & pass \\
\hline Z015 & 25.352 & 12.32 & 12.32 & 12.33 & 12.33 & 12.31 & 12.31 & $Y$ & 6.3399 & pass \\
\hline$Z 016$ & 25.232 & 12.32 & 12.31 & 12.33 & 12.33 & 12.31 & 12.31 & $Y$ & 6.3613 & pass \\
\hline Z017 & 25.063 & 12.31 & 12.31 & 12.32 & 12.31 & 12.30 & 12.30 & $Y$ & 6.3530 & pass \\
\hline 2018 & 25.172 & 12.31 & 12.31 & 12.32 & 12.31 & 12.30 & 12.30 & $Y$ & 6.3494 & pass \\
\hline Z019 & 25.215 & 12.31 & 12.30 & 12.32 & 12.32 & 12.30 & 12.30 & $Y$ & 6.3473 & pass \\
\hline 2020 & 25.232 & 12.31 & 12.31 & 12.33 & 12.33 & 12.31 & 12.31 & $Y$ & 6.3604 & pass \\
\hline 2021 & 25.073 & 12.31 & 12.30 & 12.31 & 12.32 & 12.30 & 12.31 & $Y$ & 6.3369 & pass \\
\hline 2024 & 25.151 & 12.31 & 12.30 & 12.32 & 12.32 & 12.30 & 12.31 & Y & 6.3502 & pass \\
\hline ZO25 & 25.112 & 12.31 & 12.31 & 12.32 & 12.31 & 12.32 & 12.32 & $Y$ & 6.3495 & pass \\
\hline 2026 & 25.196 & 12.31 & 12.31 & 12.32 & 12.32 & 12.31 & 12.31 & Y & 6.3415 & pass \\
\hline 2027 & 25.233 & 12.31 & 12.30 & 12.32 & 12.32 & 12.31 & 12.31 & Y & 6.3492 & pass \\
\hline 2028 & 25.134 & 12.31 & 12.31 & 12.32 & 12.32 & 12.31 & 12.31 & $Y$ & 6.3530 & pass \\
\hline$Z 029$ & 25.093 & 12.30 & 12.30 & 12.31 & 12.31 & 12.30 & 12.30 & $Y$ & 6.3447 & pass \\
\hline 2030 & 25.156 & 12.30 & 12.30 & 12.31 & 12.31 & 12.30 & 12.30 & Y & 6.3453 & pass \\
\hline Z031 & 25.134 & 12.30 & 12.30 & 12.31 & 12.31 & 12.30 & 12.30 & $Y$ & 6.3499 & pass \\
\hline Z032 & 25.201 & 12.32 & 12.32 & 12.33 & 12.33 & 12.32 & 12.32 & $Y$ & 6.3590 & pass \\
\hline 2033 & 25.093 & 12.31 & 12.31 & 12.32 & 12.32 & 12.31 & 12.31 & $Y$ & 6.3267 & pass \\
\hline Z034 & 25.221 & 12.32 & 12.32 & 12.32 & 12.32 & 12.31 & 12.32 & $Y$ & 6.3420 & pass \\
\hline Z035 & 25.303 & 12.30 & 12.30 & 12.32 & 12.32 & 12.30 & 12.30 & $Y$ & 6.3611 & pass \\
\hline 2036 & 25.224 & 12.30 & 12.30 & 12.31 & 12.31 & 12.30 & 12.30 & $Y$ & 6.3464 & pass \\
\hline 2037 & 25.134 & 12.30 & 12.30 & 12.31 & 12.32 & 12.31 & 12.30 & $Y$ & 6.3399 & pass \\
\hline 2038 & 25.132 & 12.31 & 12.31 & 12.32 & 12.32 & 12.31 & 12.31 & $Y$ & 6.3407 & pass \\
\hline 2039 & 25.185 & 12.31 & 12.31 & 12.32 & 12.33 & 12.32 & 12.31 & $Y$ & 6.3449 & pass \\
\hline 2040 & 25.204 & 12.30 & 12.30 & 12.31 & 12.31 & 12.30 & 12.30 & $Y$ & 6.3496 & pass \\
\hline
\end{tabular}

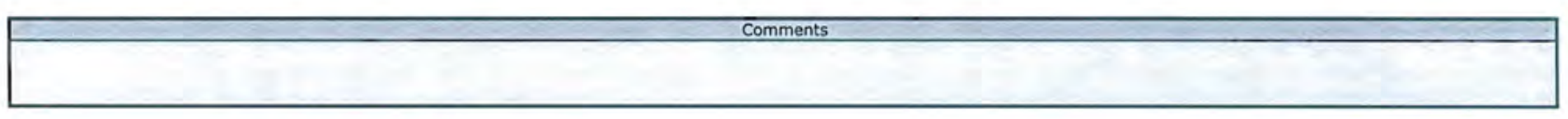

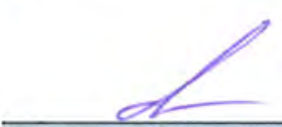

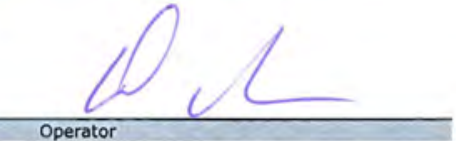

Mun

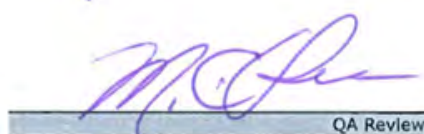

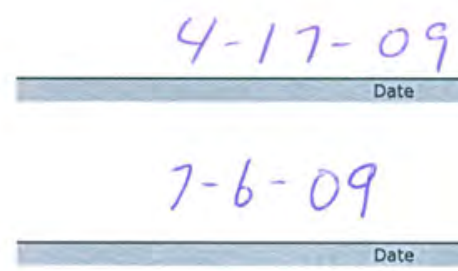

$13 / 8 / 09$ 
Data Report Form DRF-24A: Compact Diameter and Lenath

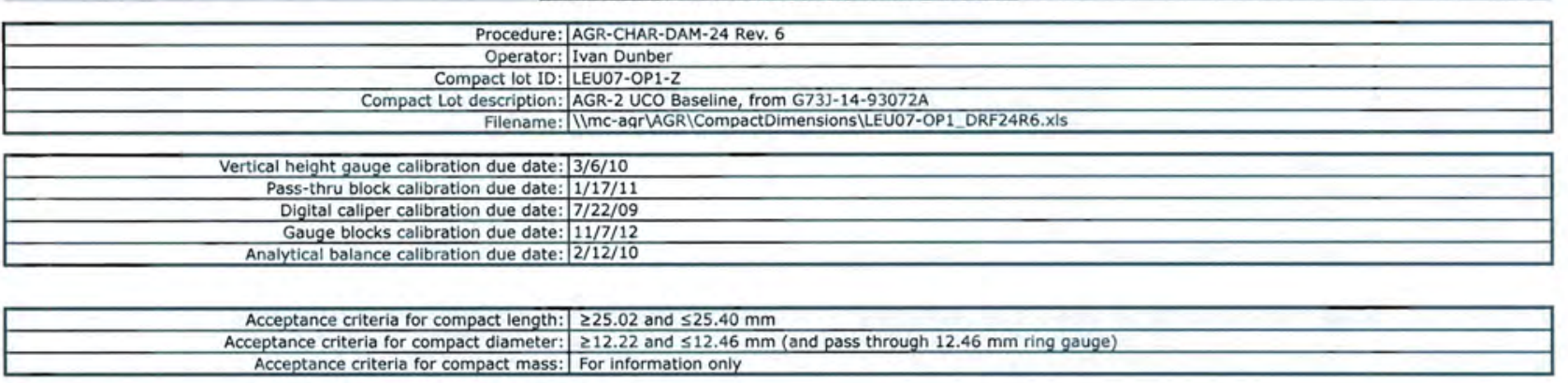

\begin{tabular}{|c|c|c|c|c|c|c|c|c|c|c|}
\hline \multirow{2}{*}{$\begin{array}{l}\text { Compact } \\
\text { ID Number }\end{array}$} & \multirow{2}{*}{$\begin{array}{l}\text { Length } \\
(\mathrm{mm})\end{array}$} & \multicolumn{6}{|c|}{ Diameter $(\mathrm{mm})$} & \multirow{2}{*}{$\begin{array}{c}\text { Pass Thru? } \\
(Y \text { or } N)\end{array}$} & \multirow{2}{*}{\begin{tabular}{l|} 
Compact weight \\
(a)
\end{tabular}} & \multirow{2}{*}{$\begin{array}{c}\text { Accept? } \\
\text { (pass or fail) }\end{array}$} \\
\hline & & Top 1 & Top 2 & Middle 1 & Middle 2 & Bottom 1 & Bottom 2 & & & \\
\hline 2041 & & 12.31 & 12.30 & 12.31 & 12.31 & 12.31 & 12.30 & & & \\
\hline 2042 & 25.226 & 12.31 & 12,31 & 12.32 & 12.33 & 12.32 & 12.31 & $Y$ & 6.3584 & pass \\
\hline 2043 & 25.063 & 12.31 & 12.30 & 12.32 & 12.31 & 12.31 & 12.31 & Y & 6.3437 & pass \\
\hline 2044 & 25.091 & 12.30 & 12.31 & 12.31 & 12.32 & 12.30 & 12.30 & $Y$ & 6.3397 & pass \\
\hline 2045 & 25.163 & 12.31 & 12.31 & 12.32 & 12.32 & 12.31 & 12.31 & $\begin{array}{l} \\
\end{array}$ & 6.3478 & pass \\
\hline 2046 & 25.076 & 12.30 & 12.30 & 12.32 & 12.32 & 12.30 & 12.30 & $Y$ & 6.3524 & pass \\
\hline 2047 & 25.205 & 12.31 & 12.32 & 12.32 & 12.32 & 12.32 & 12.32 & $\mathrm{Y}$ & 6.3480 & pass \\
\hline 2048 & 25.120 & 12.32 & 12.31 & 12.32 & 12.32 & 12.31 & 12.32 & $\mathrm{Y}$ & 6.3350 & pass \\
\hline 2049 & 25.245 & 12.31 & 12.31 & 12.32 & 12.32 & 12.30 & 12.31 & $Y$ & 6.3393 & pass \\
\hline 2050 & 25.134 & 12.31 & 12.32 & 12.34 & 12.34 & 12.31 & 12.32 & $Y$ & 6.3517 & pass \\
\hline 2051 & 25.201 & 12.31 & 12.32 & 12.32 & 12.32 & 12.30 & 12.31 & $\begin{array}{l} \\
Y\end{array}$ & 6.3400 & pass \\
\hline 2052 & 25.234 & 12.31 & 12.32 & 12.32 & 12.33 & 12.32 & 12.32 & $Y$ & 6.3528 & pass \\
\hline 2053 & 25.273 & 12.30 & 12.31 & 12.32 & 12.33 & 12.31 & 12.31 & $Y$ & 6.3563 & pass \\
\hline 2054 & 25.157 & 12.32 & 12.32 & 12.33 & 12.33 & 12.32 & 12.32 & $Y$ & 6.3552 & pass \\
\hline 2055 & 25.062 & 12.31 & 12.32 & 12.31 & 12.32 & 12.32 & 12.31 & $Y$ & 6.3535 & pass \\
\hline 2056 & 25.241 & 12.31 & 12.31 & 12.32 & 12.32 & 12.31 & 12.32 & $Y$ & 6.3569 & pass \\
\hline 2057 & 25.242 & 12.31 & 12.30 & 12.31 & 12.31 & 12.30 & 12.31 & 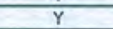 & 6.3532 & pass \\
\hline 2058 & 25.174 & 12.32 & 12.31 & 12.32 & 12.31 & 12.32 & 12.31 & $\dot{Y}$ & 6.3426 & pass \\
\hline 2059 & 25.030 & 12.30 & 12.30 & 12.31 & 12.31 & 12.31 & 12.30 & $Y$ & 6.3389 & pass \\
\hline 2060 & 25.267 & 12.32 & 12.32 & 12.33 & 12.33 & 12.33 & 12.32 & $Y$ & 6.3550 & pass \\
\hline 2061 & 25.336 & 12.33 & 12.32 & 12.33 & 12.33 & 12.33 & 12.32 & $Y$ & 6.3425 & pass \\
\hline 2062 & 25.218 & 12.31 & 12.32 & 12.32 & 12.32 & 12.30 & 12.31 & $Y$ & 6.3434 & pass \\
\hline 2063 & 25.095 & 12.32 & 12.32 & 12.33 & 12.33 & 12.32 & 12.32 & $Y$ & 6.3432 & pass \\
\hline 2064 & 25.131 & 12.32 & 12.32 & 12.32 & 12.32 & 12.31 & 12.31 & $Y$ & 6.3355 & pass \\
\hline 2065 & 25.288 & 12.32 & 12.32 & 12.33 & 12.33 & 12.32 & 12.32 & $Y$ & 6.3456 & pass \\
\hline 2066 & 25.134 & 12.33 & 12.32 & 12.33 & 12.33 & 12.32 & 12.32 & $Y$ & 6.3521 & pass \\
\hline 2067 & 25.212 & 12.31 & 12.32 & 12.33 & 12.33 & 12.31 & 12.32 & $Y$ & 6.3400 & pass \\
\hline 2068 & 25.165 & 12.31 & 12.31 & 12.32 & 12.32 & 12.32 & 12.32 & $\bar{Y}$ & 6.3575 & pass \\
\hline 2069 & 25.214 & 12.32 & 12.32 & 12.33 & 12.33 & 12.31 & 12.31 & $Y$ & 6.3450 & pass \\
\hline 2070 & 25.177 & 12.31 & 12.31 & 12.33 & 12.33 & 12.31 & 12.31 & $Y$ & 6.3416 & pass \\
\hline 2071 & 25.215 & 12.32 & 12.32 & 12.32 & 12.33 & 12.32 & 12.31 & $Y$ & 6.3573 & pass \\
\hline 2072 & 25.152 & 12.31 & 12.31 & 12.32 & 12.32 & 12.30 & 12.30 & $Y$ & 6.3374 & pass \\
\hline 2073 & 25.246 & 12.32 & 12.32 & 12.33 & 12.33 & 12.32 & 12.32 & $Y$ & 6.3604 & pass \\
\hline 2074 & 25.134 & 12.32 & 12.31 & 12.32 & 12.32 & 12.31 & 12.31 & $Y$ & 6.3449 & pass \\
\hline 2075 & 25.234 & 12.32 & 12.32 & 12.33 & 12.33 & 12.32 & 12.32 & $Y$ & 6.3514 & pass \\
\hline 2076 & 25.251 & 12.31 & 12.31 & 12.32 & 12.32 & 12.31 & 12.31 & $Y$ & 6.3512 & pass \\
\hline 2077 & 25.143 & 12.32 & 12.32 & 12.32 & 12.33 & 12.31 & 12.31 & $Y$ & 6.3576 & pass \\
\hline 2078 & 25.234 & 12.31 & 12.31 & 12.32 & 12.32 & 12.31 & 12.32 & $Y$ & 6.3293 & pass \\
\hline 2079 & 25.315 & 12.31 & 12.30 & 12.32 & 12.32 & 12.32 & 12.32 & $Y$ & 6.3491 & pass \\
\hline 2080 & 25.161 & 12.32 & 12.31 & 12.32 & 12.32 & 12.30 & 12.31 & $Y$ & 6.3478 & pass \\
\hline
\end{tabular}

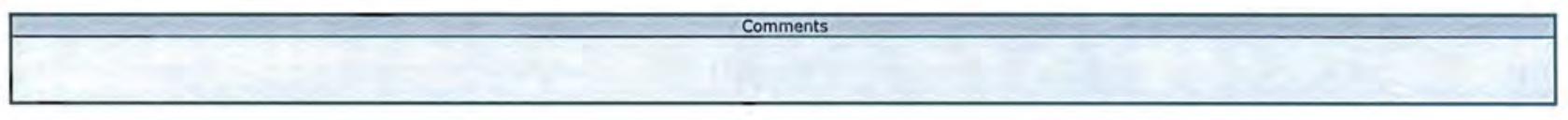

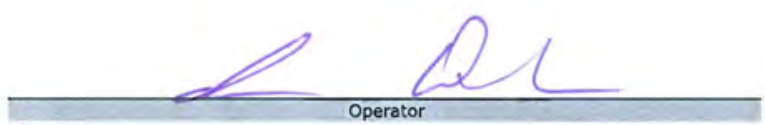

MOn actupervisor

QA Revie

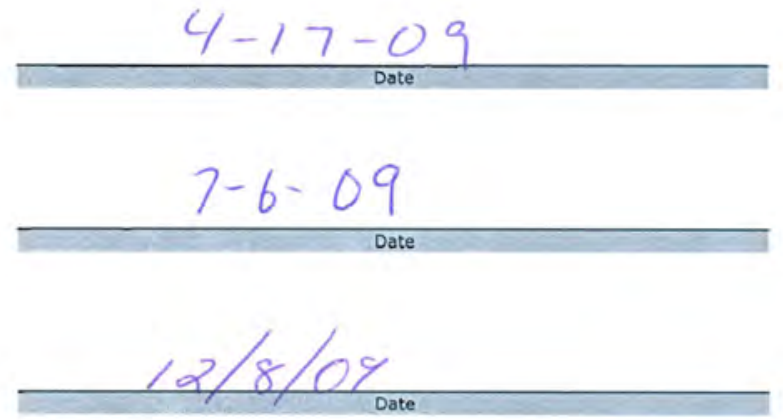


Data Report Form DRF-24A: Compact Dlameter and Length

\begin{tabular}{|c|c|}
\hline Procedure: & AGR-CHAR-DAM-24 Rev. 6 \\
\hline Operator: & Ivan Dunber \\
\hline Compact lot ID & LEU07-OP1-Z \\
\hline Compact Lot description & AGR-2 UCO Baseline, from G73J-14-93072A \\
\hline Filename & IIImc-aarVAGRICompactDimensions/LEU07-OP1_DRF24R6.xls \\
\hline Vertical height gauge calibration due date & $3 / 6 / 10$ \\
\hline Pass-thru block calibration due date & $1 / 17 / 11$ \\
\hline Digital caliper calibration due date & $7 / 22 / 09$ \\
\hline Gauge blocks calibration due date & $11 / 7 / 12$ \\
\hline Analytical balance callibration due date & $2 / 12 / 10$ \\
\hline
\end{tabular}

\begin{tabular}{|r|l|}
\hline Acceptance criteria for compact length: & $\geq 25.02$ and $\leq 25.40 \mathrm{~mm}$ \\
\hline Acceptance criteria for compact diameter: & $\geq 12.22$ and $\leq 12.46 \mathrm{~mm}$ (and pass through 12.46 mm ring gauge) \\
\hline Acceptance criteria for compact mass: & For information only \\
\hline
\end{tabular}

\begin{tabular}{|c|c|c|c|c|c|c|c|c|c|c|}
\hline \multirow{2}{*}{$\begin{array}{l}\text { Compact } \\
\text { ID Number }\end{array}$} & \multirow{2}{*}{$\begin{array}{l}\text { Length } \\
(\mathrm{mm})\end{array}$} & \multicolumn{6}{|c|}{ Diameter $(\mathrm{mm})$} & \multirow{2}{*}{$\begin{array}{l}\text { Pass Thru? } \\
(Y \circ \text { or } N)\end{array}$} & \multirow{2}{*}{$\begin{array}{l}\text { Compact weight } \\
\text { (a) }\end{array}$} & \multirow{2}{*}{$\begin{array}{c}\text { Accept? } \\
\text { (pass or fail) }\end{array}$} \\
\hline & & Top 1 & Top 2 & Middle 1 & Middle 2 & Bottom 1 & Bottom 2 & & & \\
\hline 2081 & 25.083 & 12.31 & 12.31 & 12.32 & 12.32 & 12.31 & 12.31 & $\bar{Y}$ & 6.3519 & pass \\
\hline 2082 & 25.078 & 12.31 & 12.32 & 12.31 & 12.32 & 12.31 & 12.30 & $Y$ & 6.3452 & pass \\
\hline 2083 & 25.155 & 12.31 & 12.32 & 12.32 & 12.32 & 12.31 & 12.31 & $\bar{Y}$ & 6.3436 & pass \\
\hline 2084 & 25.219 & 12.32 & 12.32 & 12.33 & 12.33 & 12.32 & 12.31 & $Y$ & 6.3638 & pass \\
\hline 2085 & 25.196 & 12.32 & 12.30 & 12.33 & 12.32 & 12.31 & 12.31 & $\begin{array}{r} \\
\end{array}$ & 6.3373 & pass \\
\hline 2086 & 25.219 & 12.32 & 12.32 & 12.33 & 12.33 & 12.32 & 12.32 & $\bar{Y}$ & 6.3558 & pass \\
\hline 2087 & 25.340 & 12.32 & 12.32 & 12.33 & 12.33 & 12.32 & 12.32 & 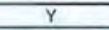 & 6.3544 & pass \\
\hline 2088 & 25.236 & 12.31 & 12.31 & 12.32 & 12.32 & 12.31 & 12.31 & $\bar{Y}$ & 6.3484 & pass \\
\hline 2089 & 25.223 & 12.32 & 12.32 & 12.33 & 12.33 & 12.32 & 12.32 & $Y$ & 6.3548 & pass \\
\hline Z091 & 25.115 & 12.32 & 12.32 & 12.33 & 12.33 & 12.32 & 12.31 & $\bar{Y}$ & 6.3576 & pass \\
\hline 2092 & 25.147 & 12.30 & 12.30 & 12.32 & 12.32 & 12.30 & 12.31 & $Y$ & 6.3390 & pass \\
\hline Z093 & 25.163 & 12.31 & 12.31 & 12.32 & 12.32 & 12.31 & 12.31 & $Y$ & 6.3559 & pass \\
\hline Z094 & 25.135 & 12.31 & 12.31 & 12.32 & 12.32 & 12.31 & 12.31 & $\bar{Y}$ & 6.3409 & pass \\
\hline 2095 & 25.233 & 12.31 & 12.31 & 12.33 & 12.33 & 12.32 & 12.32 & $Y$ & 6.3616 & pass \\
\hline Z096 & 25.252 & 12.31 & 12.31 & 12.32 & 12.32 & 12.30 & 12.31 & $\bar{Y}$ & 6.3530 & pass \\
\hline 2097 & 25.185 & 12.31 & 12.31 & 12.32 & 12.33 & 12.32 & 12.31 & $Y$ & 6.3451 & pass \\
\hline 2098 & 25.195 & 12.32 & 12.31 & 12.33 & 12.33 & 12.32 & 12.32 & $\bar{Y}$ & 6.3492 & pass \\
\hline Z099 & 25.206 & 12.32 & 12.31 & 12.33 & 12.32 & 12.30 & 12.31 & $Y$ & 6.3549 & pass \\
\hline $\mathrm{Z100}$ & 25.142 & 12.31 & 12.31 & 12.33 & 12.32 & 12.32 & 12.31 & $Y$ & 6.3409 & pass \\
\hline$Z 101$ & 25.146 & 12.30 & 12.30 & 12.32 & 12.32 & 12.30 & 12.31 & $\mathrm{Y}$ & 6.3309 & pass \\
\hline $\mathrm{Z} 104$ & 25.077 & 12.30 & 12.31 & 12.32 & 12.32 & 12.30 & 12.30 & $Y$ & 6.3266 & pass \\
\hline Z105 & 25.133 & 12.30 & 12.30 & 12.32 & 12.32 & 12.31 & 12.31 & $Y$ & 6.3386 & pass \\
\hline Z106 & 25.184 & 12.30 & 12.31 & 12.32 & 12.33 & 12.30 & 12.31 & $Y$ & 6.3277 & pass \\
\hline$Z 107$ & 25.175 & 12.31 & 12.31 & 12.33 & 12.33 & 12.31 & 12.32 & $Y$ & 6.3457 & pass \\
\hline$Z 108$ & 25.151 & 12.31 & 12.31 & 12.33 & 12.33 & 12.32 & 12.32 & $Y$ & 6.3570 & pass \\
\hline Z109 & 25.194 & 12.32 & 12.32 & 12.34 & 12.33 & 12.32 & 12.31 & $Y$ & 6.3308 & pass \\
\hline$Z 110$ & 25.032 & 12.31 & 12.31 & 12.32 & 12.33 & 12.31 & 12.31 & $Y$ & 6.3430 & pass \\
\hline$Z 111$ & 25.238 & 12.31 & 12.32 & 12.32 & 12.33 & 12.31 & 12.31 & $Y$ & 6.3402 & pass \\
\hline$Z 112$ & 25.217 & 12.30 & 12.30 & 12.32 & 12.32 & 12.30 & 12.31 & $Y$ & 6.3430 & pass \\
\hline $\mathrm{Z113}$ & 25.177 & 12.30 & 12.30 & 12.32 & 12.31 & 12.30 & 12.31 & $Y$ & 6.3466 & pass \\
\hline $\mathrm{Z114}$ & 25.142 & 12.31 & 12.30 & 12.32 & 12.31 & 12.30 & 12.31 & $Y$ & 6.3289 & pass \\
\hline$Z 115$ & 25.192 & 12.31 & 12.32 & 12.32 & 12.32 & 12.31 & 12.31 & $Y$ & 6.3347 & pass \\
\hline$Z 116$ & 25.278 & 12.32 & 12.32 & 12.33 & 12.33 & 12.32 & 12.32 & $Y$ & 6.3665 & pass \\
\hline$Z 117$ & 25.135 & 12.30 & 12.30 & 12.32 & 12.32 & 12.31 & 12.31 & $\bar{Y}$ & 6.3332 & pass \\
\hline Z118 & 25.228 & 12.30 & 12.31 & 12.32 & 12.32 & 12.31 & 12.31 & $Y$ & 6.3454 & pass \\
\hline Z119 & 25.112 & 12.32 & 12.31 & 12.32 & 12.32 & 12.30 & 12.30 & $Y$ & 6.3501 & pass \\
\hline $\mathrm{Z} 120$ & 25.298 & 12.32 & 12.31 & 12.32 & 12.32 & 12.30 & 12.31 & $Y$ & 6.3380 & pass \\
\hline
\end{tabular}

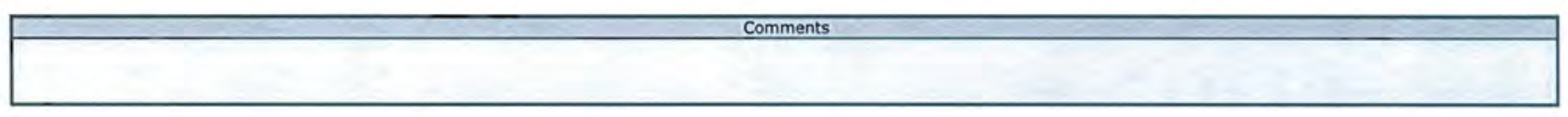

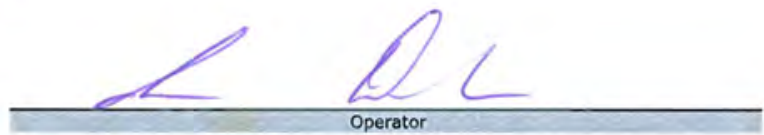
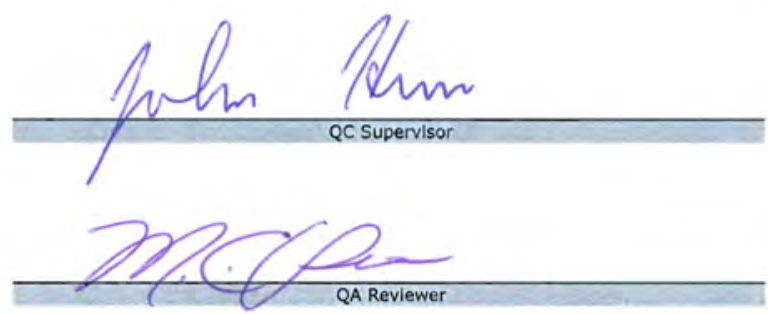

$$
4-17-09
$$$$
7-6-09
$$
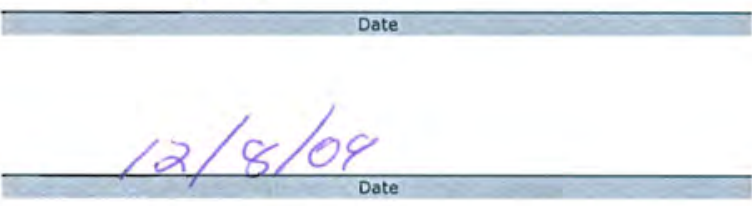
ORNL/TM-2009/305
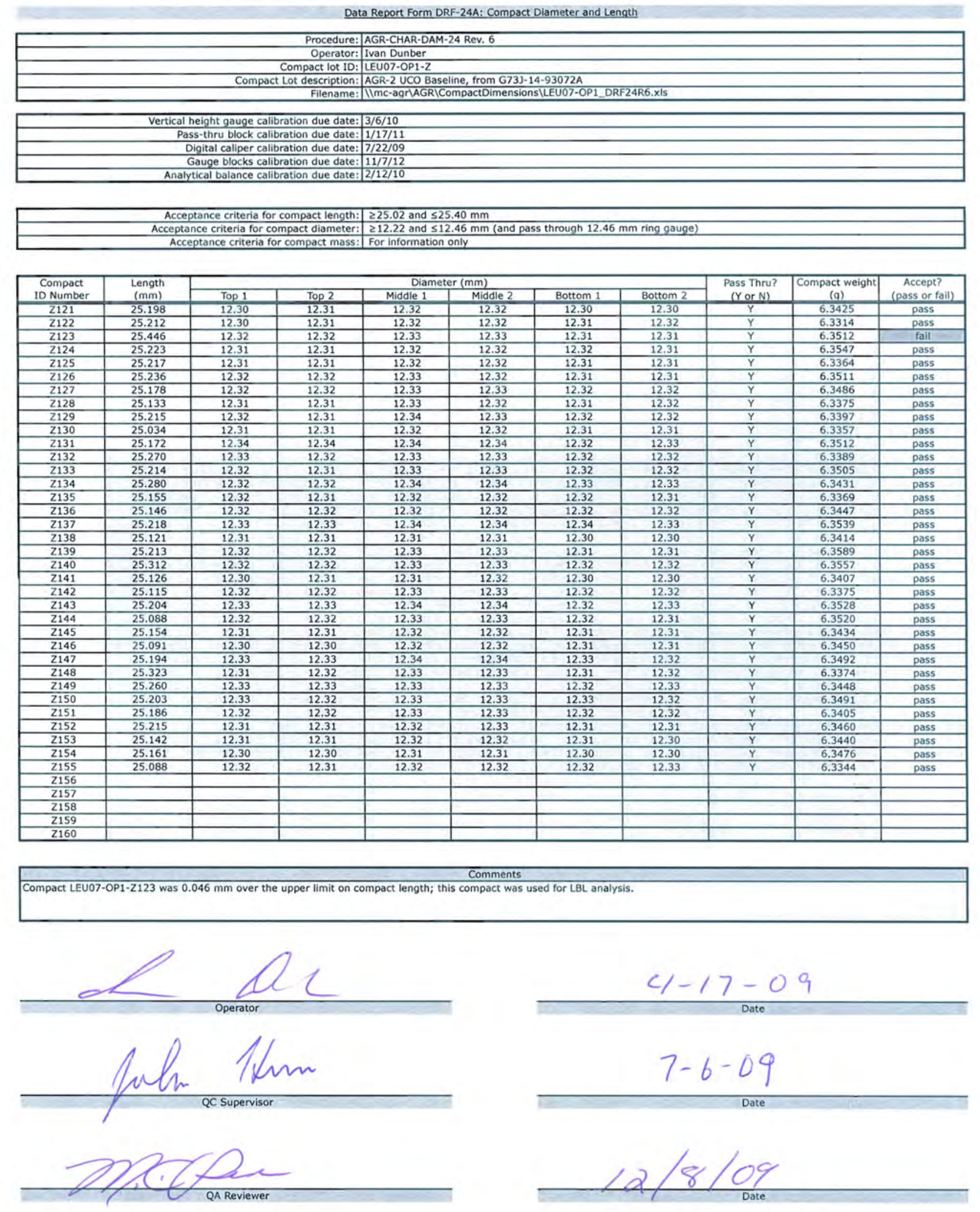

61 
ORNL/TM-2009/305
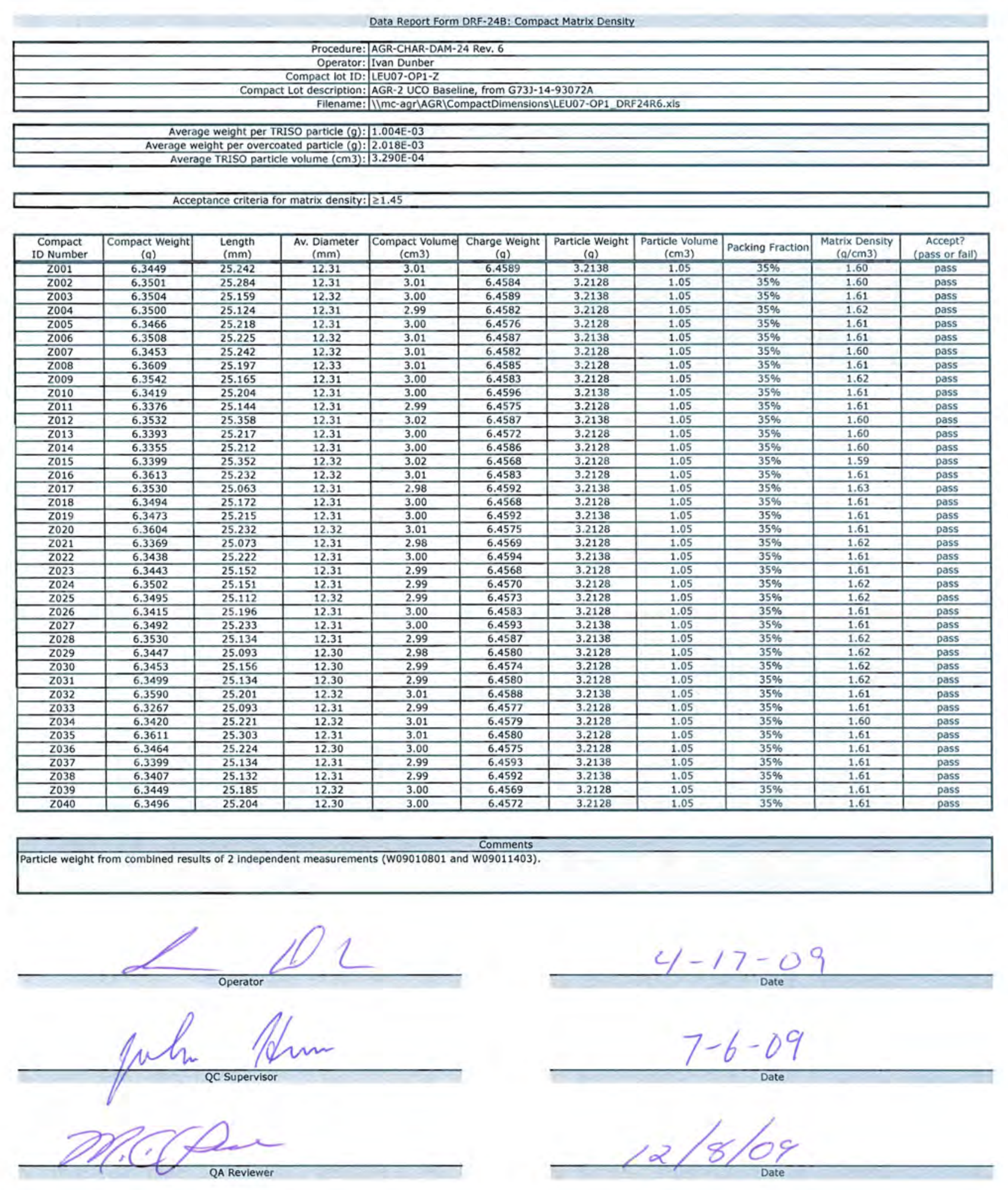

62 
Data Report Form DRF-24B: Compact Matrix Density

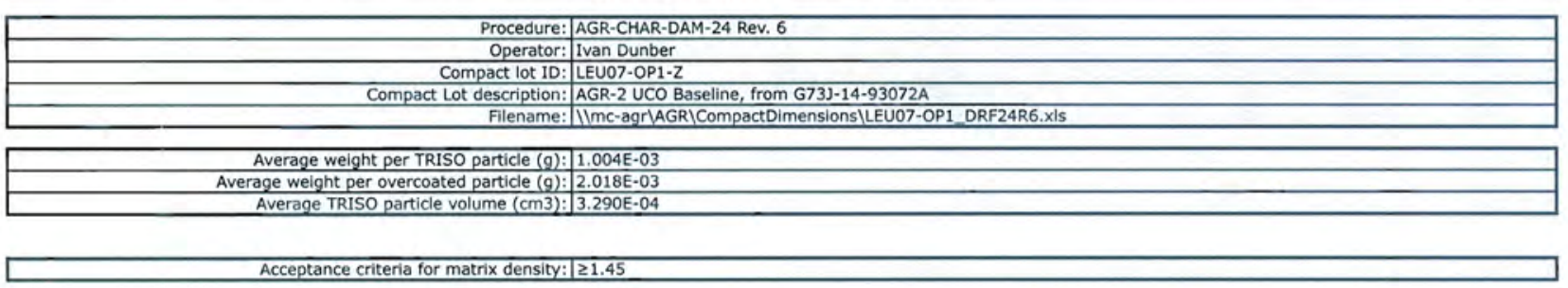

\begin{tabular}{|c|c|c|c|c|c|c|c|c|c|c|}
\hline $\begin{array}{l}\text { Compact } \\
\text { ID Number }\end{array}$ & $\begin{array}{l}\text { Compact Weight } \\
\text { (a) }\end{array}$ & $\begin{array}{l}\text { Length } \\
(\mathrm{mm})\end{array}$ & $\begin{array}{l}\text { Av. Diameter } \\
(\mathrm{mm})\end{array}$ & $\begin{array}{l}\text { Compact Volume } \\
(\mathrm{cm} 3)\end{array}$ & $\begin{array}{l}\text { Charge Weight } \\
\text { (a) }\end{array}$ & $\begin{array}{l}\text { Particle Weight } \\
\text { (a) }\end{array}$ & $\begin{array}{l}\text { Particle Volume } \\
\text { (cm3) }\end{array}$ & \begin{tabular}{|l|} 
Packing Fraction \\
\end{tabular} & $\begin{array}{l}\text { Matrix Density } \\
(\mathrm{a} / \mathrm{cm} 3)\end{array}$ & $\begin{array}{c}\text { Accept? } \\
\text { (pass or fail) }\end{array}$ \\
\hline 2041 & 6.3464 & 25.121 & 12.31 & 2.99 & 6.4576 & 3.2128 & 1.05 & $35 \%$ & 1.62 & pass \\
\hline 2042 & 6.3584 & 25.226 & 12.32 & 3.01 & 6.4564 & 3.2118 & 1.05 & $35 \%$ & 1.61 & pass \\
\hline 2043 & 6.3437 & 25.063 & 12.31 & 2.98 & 6.4593 & 3.2138 & 1.05 & $35 \%$ & 1.62 & pass \\
\hline 2044 & 6.3397 & 25.091 & 12.31 & 2.98 & 6.4595 & 3.2138 & 1.05 & $35 \%$ & 1.62 & pass \\
\hline 2045 & 6.3478 & 25.163 & 12.31 & 3.00 & 6.4594 & 3.2138 & 1.05 & $35 \%$ & 1.61 & pass \\
\hline 2046 & 6.3524 & 25.076 & 12.31 & 2.98 & 6.4577 & 3.2128 & 1.05 & $35 \%$ & 1.63 & pass \\
\hline Z047 & 6.3480 & 25.205 & 12.32 & 3.00 & 6.4572 & 3.2128 & 1.05 & $35 \%$ & 1.61 & pass \\
\hline 2048 & 6.3350 & 25.120 & 12.32 & 2.99 & 6.4583 & 3.2128 & 1.05 & $35 \%$ & 1.61 & pass \\
\hline Z049 & 6.3393 & 25.245 & 12.31 & 3.01 & 6.4585 & 3.2128 & 1.05 & $35 \%$ & 1.60 & pass \\
\hline 2050 & $\frac{0.353}{6.3517}$ & $\frac{25.245}{25.134}$ & $\frac{12.31}{12.32}$ & $\frac{3.01}{3.00}$ & $\frac{0.4303}{6.4590}$ & 3.2138 & 1.05 & $35 \%$ & 1.61 & $\frac{p \text { pass }}{\text { pass }}$ \\
\hline Z051 & 6.3400 & 25.201 & 12.31 & 3.00 & 6.4579 & 3.2128 & 1.05 & $35 \%$ & 1.61 & pass \\
\hline 2052 & 6.3528 & 25.234 & 12.32 & 3.01 & 6.4574 & 3.2128 & 1.05 & $35 \%$ & 1.61 & pass \\
\hline 2053 & 6.3563 & 25.273 & 12.31 & 3.01 & 6.4597 & 3.2138 & 1.05 & $35 \%$ & 1.61 & pass \\
\hline 2054 & 6.3552 & 25.157 & 12.32 & 3.00 & 6.4580 & 3.2128 & 1.05 & $35 \%$ & 1.61 & pass \\
\hline 2055 & 6.3535 & 25.062 & 12.32 & 2.99 & 6.4581 & 3.2128 & 1.05 & $35 \%$ & 1.63 & pass \\
\hline 2056 & 6.3569 & 25.241 & 12.32 & 3.01 & 6.4589 & 3.2138 & 1.05 & $35 \%$ & 1.61 & pass \\
\hline 2057 & 6.3532 & 25.242 & 12.31 & 3.00 & 6.4575 & 3.2128 & 1.05 & $35 \%$ & 1.61 & pass \\
\hline 2058 & 6.3426 & 25.174 & 12.32 & 3.00 & 6.4591 & 3.2138 & 1.05 & $35 \%$ & 1.61 & pass \\
\hline 2059 & 6.3389 & 25.030 & 12.31 & 2.98 & 6.4583 & 3.2128 & 1.05 & $35 \%$ & 1.62 & pass \\
\hline 2060 & 6.3550 & 25.267 & 12.33 & 3.01 & 6.4583 & 3.2128 & 1.05 & $35 \%$ & 1.60 & pass \\
\hline 2061 & 6.3425 & 25.336 & 12.33 & 3.02 & 6.4581 & 3.2128 & 1.05 & $35 \%$ & 1.59 & pass \\
\hline 2062 & 6.3434 & 25.218 & 12.31 & 3.00 & 6.4575 & 3.2128 & 1.05 & $35 \%$ & 1.61 & pass \\
\hline Z063 & 6.3432 & 25.095 & 12.32 & 2.99 & 6.4593 & 3.2138 & 1.05 & $35 \%$ & 1.61 & pass \\
\hline 2064 & 6.3355 & 25.131 & 12.32 & 2.99 & 6.4579 & 3.2128 & 1.05 & $35 \%$ & 1.61 & pass \\
\hline 2065 & 6.3456 & 25.288 & 12.32 & 3.02 & 6.4586 & 3.2128 & 1.05 & $35 \%$ & 1.60 & pass \\
\hline 2066 & 6.3521 & 25.134 & 12.33 & 3.00 & 6.4591 & 3.2138 & 1.05 & $35 \%$ & 1.61 & pass \\
\hline 2067 & 6.3400 & 25.212 & 12.32 & 3.01 & 6.4585 & 3.2128 & 1.05 & $35 \%$ & 1.60 & pass \\
\hline 2068 & 6.3575 & 25.165 & 12.32 & 3.00 & 6.4587 & 3.2138 & 1.05 & $35 \%$ & 1.62 & pass \\
\hline 2069 & 6.3450 & 25.214 & 12.32 & 3.01 & 6.4582 & 3.2128 & 1.05 & $35 \%$ & 1.60 & pass \\
\hline 2070 & 6.3416 & 25.177 & 12.32 & 3.00 & $\frac{0.4502}{6.4572}$ & $\frac{3.428}{3.2128}$ & 1.05 & $35 \%$ & 1.61 & $\frac{\text { pass }}{\text { pass }}$ \\
\hline Z071 & 6.3573 & 25.215 & 12.32 & 3.01 & 6.4591 & 3.2138 & 1.05 & $35 \%$ & 1.61 & pass \\
\hline 2072 & 6.3374 & 25.152 & 12.31 & 2.99 & 6.4590 & 3.2138 & 1.05 & $35 \%$ & 1.61 & pass \\
\hline 2073 & 6.3604 & 25.246 & 12.32 & 3.01 & 6.4583 & 3.2128 & 1.05 & $35 \%$ & 1.61 & pass \\
\hline 2074 & 6.3449 & 25.134 & 12.32 & 2.99 & 6.4570 & 3.2128 & 1.05 & $35 \%$ & 1.61 & pass \\
\hline 2075 & 6.3514 & 25.234 & 12.32 & 3.01 & 6.4575 & 3.2128 & 1.05 & $35 \%$ & 1.60 & pass \\
\hline Z076 & 6.3512 & 25.251 & 12.31 & 3.01 & 6.4 & 3.2 & 1.05 & $35 \%$ & 1.61 & pass \\
\hline 2077 & 6.3576 & 25.143 & 12.32 & 3.00 & 6.4595 & 3.2138 & 1.05 & $35 \%$ & 1.62 & pass \\
\hline Z078 & 6.3293 & 25.234 & 12.32 & 3.01 & 6.4592 & 3.2138 & 1.05 & $35 \%$ & 1.60 & pass \\
\hline 2079 & 6.3491 & 25.315 & 12.32 & 3.02 & 6.4587 & 3.2138 & 1.05 & $35 \%$ & 1.60 & pass \\
\hline 2080 & 6.3478 & 25.161 & 12.31 & 3.00 & 6.4597 & 3.2138 & 1.05 & $35 \%$ & 1.61 & pass \\
\hline
\end{tabular}

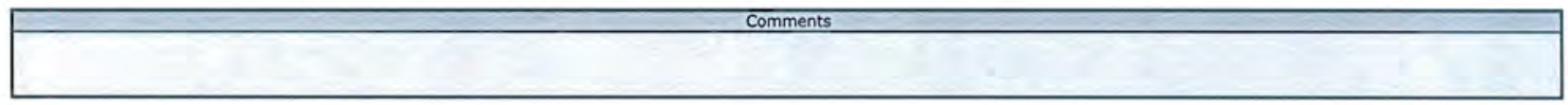

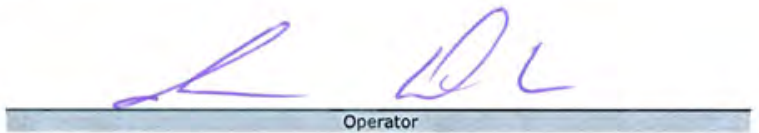
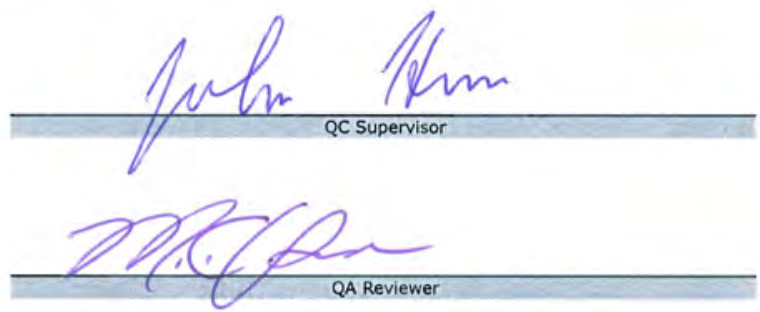

$$
4-17-09
$$

$$
7-6-09
$$
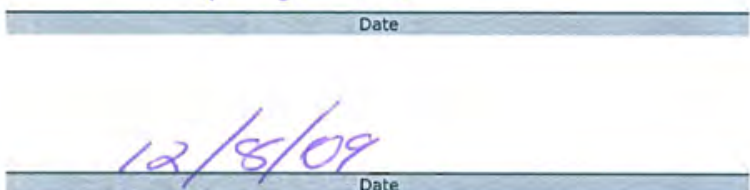


\begin{tabular}{|c|c|c|c|c|c|c|c|c|c|c|}
\hline $\begin{array}{l}\text { Compact } \\
\text { ID Number }\end{array}$ & \begin{tabular}{|c|} 
Compact Weight \\
(a)
\end{tabular} & $\begin{array}{l}\text { Length } \\
(\mathrm{mm})\end{array}$ & $\begin{array}{l}\text { Av. Diameter } \\
(\mathrm{mm})\end{array}$ & $\begin{array}{c}\begin{array}{c}\text { Compact Volume } \\
(\mathrm{cm} 3)\end{array} \\
\end{array}$ & $\begin{array}{l}\text { Charge Weight } \\
\text { (a) }\end{array}$ & $\begin{array}{c}\text { Particle Weight } \\
\text { (a) }\end{array}$ & $\begin{array}{c}\begin{array}{c}\text { Particle Volume } \\
(\mathrm{cm} 3)\end{array} \\
\end{array}$ & Packing Fraction & $\begin{array}{l}\text { Matrix Density } \\
(\mathrm{a} / \mathrm{cm} 3)\end{array}$ & $\begin{array}{c}\text { Accept? } \\
\text { (pass or fail) }\end{array}$ \\
\hline 2081 & 6.3519 & 25.083 & 12.31 & 2.99 & 6.4591 & 3.2138 & 1.05 & $35 \%$ & 1.62 & pass \\
\hline 2082 & 6.3452 & 25.078 & 12.31 & 2.99 & 6.4583 & 3.2128 & 1.05 & $35 \%$ & 1.62 & pass \\
\hline 2083 & 6.3436 & 25.155 & 12.32 & 3.00 & 6.4588 & 3.2138 & 1.05 & $35 \%$ & 1.61 & pass \\
\hline Z084 & 6.3638 & 25.219 & 12.32 & 3.01 & 6.4586 & 3.2128 & 1.05 & $35 \%$ & 1.61 & pass \\
\hline 2085 & 6.3373 & 25.196 & 12.32 & 3.00 & 6.4583 & 3.2128 & 1.05 & $35 \%$ & 1.60 & pass \\
\hline 2086 & 6.3558 & 25.219 & 12.32 & 3.01 & 6.4568 & 3.2128 & 1.05 & $35 \%$ & 1.61 & pass \\
\hline 2087 & 6.3544 & 25.340 & 12.32 & 3.02 & 6.4593 & 3.2138 & 1.05 & $35 \%$ & 1.59 & pass \\
\hline 2088 & 6.3484 & 25.236 & 12.31 & 3.01 & 6.4585 & 3.2128 & 1.05 & $35 \%$ & 1.61 & pass \\
\hline 2089 & 6.3548 & 25.223 & 12.32 & 3.01 & 6.4599 & 3.2138 & 1.05 & $35 \%$ & 1.61 & pass \\
\hline 2090 & 6.3432 & 25.181 & 12.31 & 3.00 & 6.4574 & 3.2128 & 1.05 & $35 \%$ & 1.61 & pass \\
\hline 2091 & 6.3576 & 25.115 & 12.32 & 2.99 & 6.4570 & 3.2128 & 1.05 & $35 \%$ & 1.62 & pass \\
\hline ZO92 & 6.3390 & 25.147 & 12.31 & 2.99 & 6.4579 & 3.2128 & 1.05 & $35 \%$ & 1.61 & pass \\
\hline 2093 & 6.3559 & 25.163 & 12.31 & 3.00 & 6.4586 & 3.2128 & 1.05 & $35 \%$ & 1.62 & pass \\
\hline 2094 & 6.3409 & 25.135 & 12.31 & 2.99 & 6.4578 & 3.2128 & 1.05 & $35 \%$ & 1.61 & pass \\
\hline 2095 & 6.3616 & 25.233 & 12.32 & 3.01 & 6.4593 & 3.2138 & 1.05 & $35 \%$ & 1.61 & pass \\
\hline 2096 & 6.3530 & 25.252 & 12.31 & 3.01 & 6.4592 & 3.2138 & 1.05 & $35 \%$ & 1.61 & pass \\
\hline 2097 & 6.3451 & 25.185 & 12.32 & 3.00 & 6.4579 & 3.2128 & 1.05 & $35 \%$ & 1.61 & pass \\
\hline 2098 & 6.3492 & 25.195 & 12.32 & 3.00 & 6.4577 & 3.2128 & 1.05 & $35 \%$ & 1.61 & pass \\
\hline Z099 & 6.3549 & 25.206 & 12.32 & 3.00 & 6.4586 & 3.2128 & 1.05 & $35 \%$ & 1.61 & pass \\
\hline 2100 & 6.3409 & 25.142 & 12.32 & 3.00 & 6.4587 & 3.2138 & 1.05 & $35 \%$ & 1.61 & pass \\
\hline $\mathrm{Z} 101$ & 6.3309 & 25.146 & 12.31 & 2.99 & 6.4582 & 3.2128 & 1.05 & $35 \%$ & 1.61 & pass \\
\hline $\mathrm{Z} 102$ & 6.3570 & 25.336 & 12.32 & 3.02 & 6.4582 & 3.2128 & 1.05 & $35 \%$ & 1.60 & pass \\
\hline $\mathrm{Z103}$ & 6.3386 & 25.195 & 12.33 & 3.01 & 6.4579 & 3.2128 & 1.05 & $35 \%$ & 1.60 & pass \\
\hline$Z 104$ & 6.3266 & 25.077 & 12.31 & 2.98 & 6.4585 & 3.2128 & 1.05 & $35 \%$ & 1.61 & pass \\
\hline $\mathrm{Z} 105$ & 6.3386 & 25.133 & 12.31 & 2.99 & 6.4593 & 3.2138 & 1.05 & $35 \%$ & 1.61 & pass \\
\hline $\mathrm{Z106}$ & 6.3277 & 25.184 & 12.31 & 3.00 & 6.4590 & 3.2138 & 1.05 & $35 \%$ & 1.60 & pass \\
\hline$Z 107$ & 6.3457 & 25.175 & 12.32 & 3.00 & 6.4576 & 3.2128 & 1.05 & $35 \%$ & 1.61 & pass \\
\hline $\mathrm{Z} 108$ & 6.3570 & 25.151 & 12.32 & 3.00 & 6.4581 & 3.2128 & 1.05 & $35 \%$ & 1.62 & pass \\
\hline$Z 109$ & 6.3308 & 25.194 & 12.32 & 3.00 & 6.4574 & 3.2128 & 1.05 & $35 \%$ & 1.60 & pass \\
\hline$Z 110$ & 6.3430 & 25.032 & 12.32 & 2.98 & 6.4582 & 3.2128 & 1.05 & $35 \%$ & 1.62 & pass \\
\hline Z111 & 6.3402 & 25.238 & 12.32 & 3.01 & 6.4587 & 3.2138 & 1.05 & $35 \%$ & 1.60 & pass \\
\hline$Z 112$ & 6.3430 & 25.217 & 12.31 & 3.00 & 6.4578 & 3.2128 & 1.05 & $35 \%$ & 1.61 & pass \\
\hline 2113 & 6.3466 & 25.177 & 12.31 & 2.99 & 6.4580 & 3.2128 & 1.05 & $35 \%$ & 1.61 & pass \\
\hline 2114 & 6.3289 & 25.142 & 12.31 & 2.99 & 6.4589 & 3.2138 & 1.05 & $35 \%$ & 1.61 & pass \\
\hline$Z 115$ & 6.3347 & 25.192 & 12.32 & 3.00 & 6.4571 & 3.2128 & 1.05 & $35 \%$ & 1.60 & pass \\
\hline$Z 116$ & 6.3665 & 25.278 & 12.32 & 3.02 & 6.4580 & 3.2128 & 1.05 & $35 \%$ & 1.61 & pass \\
\hline$Z 117$ & 6.3332 & 25.135 & 12.31 & 2.99 & 6.4581 & 3.2128 & 1.05 & $35 \%$ & 1.61 & pass \\
\hline$Z 118$ & 6.3454 & 25.228 & 12.31 & 3.00 & 6.4575 & 3.2128 & 1.05 & $35 \%$ & 1.61 & pass \\
\hline 2119 & 6.3501 & 25.112 & 12.31 & 2.99 & 6.4579 & 3.2128 & 1.05 & $35 \%$ & 1.62 & pass \\
\hline $\mathrm{Z120}$ & 6.3380 & 25.298 & 12.31 & 3.01 & 6.4582 & 3.2128 & 1.05 & $35 \%$ & 1.59 & pass \\
\hline
\end{tabular}

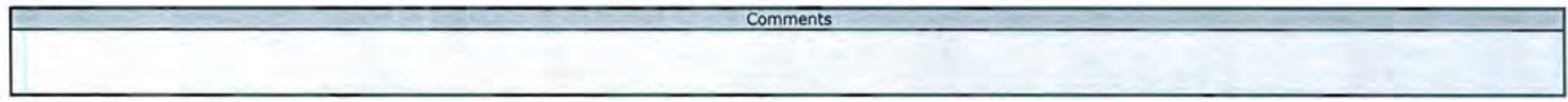

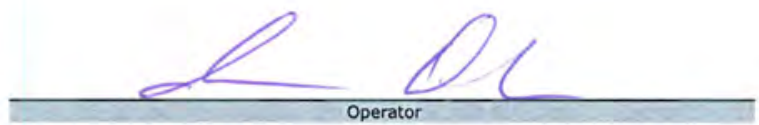
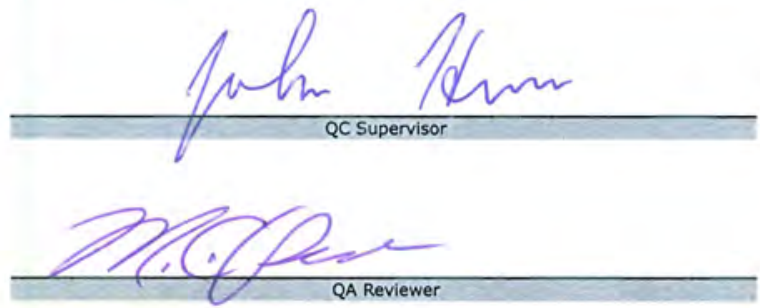

$4-17-09$

$$
7-6-09
$$

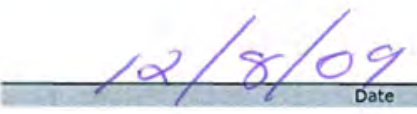




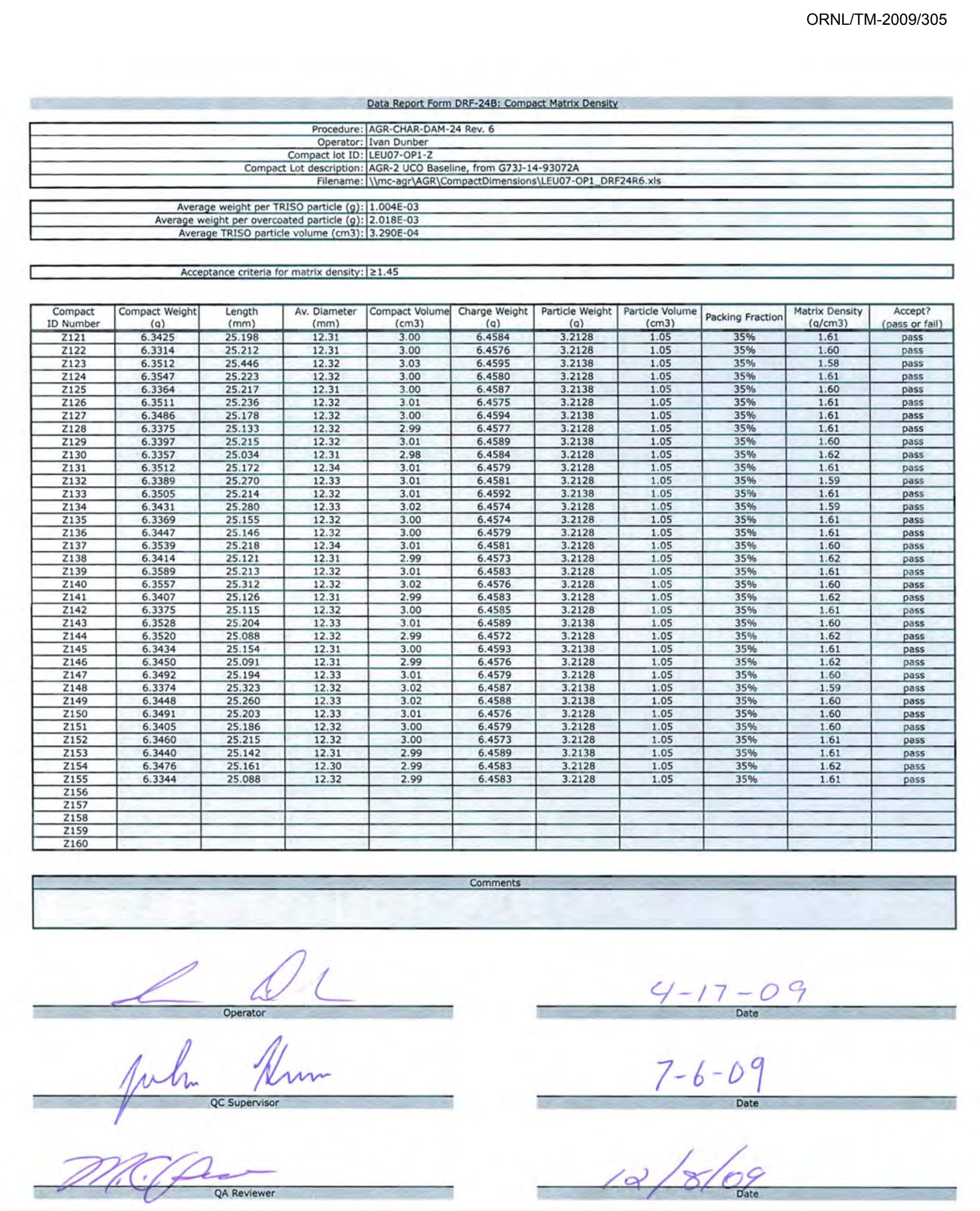


Data Report Form DRF-24C: Compact Tracking

\begin{tabular}{|r|l|}
\hline Procedure: & AGR-CHAR-DAM-24 Rev. 6 \\
\hline Operator: & Ivan Dunber \\
\hline Compact lot ID: & LEU07-OP1-Z \\
\hline Compact Lot description: & AGR-2 UCO Baseline, from G73J-14-93072A \\
\hline Filename: & \ImC-agr\AGR\CompactDimensions\LEU07-OP1_DRF24R6.xIs \\
\hline
\end{tabular}

\begin{tabular}{|c|c|}
\hline $\begin{array}{c}\text { Compact } \\
\text { Z Number }\end{array}$ & $\begin{array}{c}\text { Compact G } \\
\text { Number }\end{array}$ \\
\hline Z001 & G056 \\
\hline Z002 & G049 \\
\hline Z003 & G147 \\
\hline Z004 & G156 \\
\hline Z005 & G132 \\
\hline Z006 & G087 \\
\hline Z007 & G136 \\
\hline Z008 & G152 \\
\hline Z009 & G048 \\
\hline Z010 & G118 \\
\hline Z011 & G010 \\
\hline Z012 & G054 \\
\hline Z013 & G119 \\
\hline Z014 & G021 \\
\hline Z015 & G012 \\
\hline Z016 & G047 \\
\hline Z017 & G107 \\
\hline Z018 & G170 \\
\hline Z019 & G043 \\
\hline Z020 & G130 \\
\hline Z021 & G164 \\
\hline Z022 & G091 \\
\hline Z023 & G161 \\
\hline Z024 & G064 \\
\hline Z025 & G085 \\
\hline Z026 & G101 \\
\hline Z027 & G150 \\
\hline Z028 & G109 \\
\hline Z029 & G040 \\
\hline Z030 & G105 \\
\hline Z031 & G097 \\
\hline Z032 & G090 \\
\hline Z033 & G026 \\
\hline Z034 & G066 \\
\hline Z035 & G044 \\
\hline Z036 & G163 \\
\hline Z037 & G059 \\
\hline Z038 & G079 \\
\hline Z039 & G067 \\
\hline Z040 & G179 \\
\hline
\end{tabular}

\begin{tabular}{|c|c|}
\hline $\begin{array}{c}\text { Compact } \\
\text { Z Number }\end{array}$ & $\begin{array}{c}\text { Compact G } \\
\text { Number }\end{array}$ \\
\hline \hline Z041 & G102 \\
\hline Z042 & G157 \\
\hline Z043 & G100 \\
\hline Z044 & G008 \\
\hline Z045 & G074 \\
\hline Z046 & G103 \\
\hline Z047 & G138 \\
\hline Z048 & G072 \\
\hline Z049 & G020 \\
\hline Z050 & G149 \\
\hline Z051 & G032 \\
\hline Z052 & G165 \\
\hline Z053 & G131 \\
\hline Z054 & G083 \\
\hline Z055 & G098 \\
\hline Z056 & G045 \\
\hline Z057 & G177 \\
\hline Z058 & G146 \\
\hline Z059 & G029 \\
\hline Z060 & G082 \\
\hline Z061 & G057 \\
\hline Z062 & G060 \\
\hline Z063 & G080 \\
\hline Z064 & G073 \\
\hline Z065 & G038 \\
\hline Z066 & G076 \\
\hline Z067 & G144 \\
\hline Z068 & G110 \\
\hline Z069 & G070 \\
\hline Z070 & G078 \\
\hline Z071 & G086 \\
\hline Z072 & G116 \\
\hline Z073 & G111 \\
\hline Z074 & G051 \\
\hline Z075 & G180 \\
\hline Z076 & G151 \\
\hline Z077 & G046 \\
\hline Z078 & G031 \\
\hline Z079 & G127 \\
\hline Z080 & G171 \\
\hline & \\
\hline & \\
\hline
\end{tabular}

\begin{tabular}{|c|c|}
\hline $\begin{array}{c}\text { Compact } \\
\text { Z Number }\end{array}$ & $\begin{array}{c}\text { Compact G } \\
\text { Number }\end{array}$ \\
\hline Z081 & G104 \\
\hline Z082 & G094 \\
\hline Z083 & G142 \\
\hline Z084 & G167 \\
\hline Z085 & G115 \\
\hline Z086 & G129 \\
\hline Z087 & G068 \\
\hline Z088 & G137 \\
\hline Z089 & G140 \\
\hline Z090 & G134 \\
\hline Z091 & G112 \\
\hline Z092 & G124 \\
\hline Z093 & G106 \\
\hline Z094 & G113 \\
\hline Z095 & G172 \\
\hline Z096 & G153 \\
\hline Z097 & G071 \\
\hline Z098 & G162 \\
\hline Z099 & G108 \\
\hline Z100 & G077 \\
\hline Z101 & G033 \\
\hline Z102 & G052 \\
\hline Z103 & G154 \\
\hline Z104 & G023 \\
\hline Z105 & G037 \\
\hline Z106 & G019 \\
\hline Z107 & G143 \\
\hline Z108 & G159 \\
\hline Z109 & G025 \\
\hline Z110 & G093 \\
\hline Z111 & G022 \\
\hline Z112 & G009 \\
\hline Z113 & G062 \\
\hline Z114 & G121 \\
\hline Z115 & G141 \\
\hline Z116 & G174 \\
\hline Z117 & G122 \\
\hline Z118 & G158 \\
\hline Z119 & G095 \\
\hline Z120 & G024 \\
\hline
\end{tabular}

\begin{tabular}{|c|c|}
\hline $\begin{array}{c}\text { Compact } \\
\text { Z Number }\end{array}$ & $\begin{array}{c}\text { Compact G } \\
\text { Number }\end{array}$ \\
\hline Z121 & G042 \\
\hline Z122 & G035 \\
\hline Z123 & G041 \\
\hline Z124 & G173 \\
\hline Z125 & G034 \\
\hline Z126 & G039 \\
\hline Z127 & G148 \\
\hline Z128 & G123 \\
\hline Z129 & G028 \\
\hline Z130 & G013 \\
\hline Z131 & G084 \\
\hline Z132 & G099 \\
\hline Z133 & G061 \\
\hline Z134 & G128 \\
\hline Z135 & G120 \\
\hline Z136 & G178 \\
\hline Z137 & G135 \\
\hline Z138 & G063 \\
\hline Z139 & G175 \\
\hline Z140 & G053 \\
\hline Z141 & G117 \\
\hline Z142 & G155 \\
\hline Z143 & G145 \\
\hline Z144 & G092 \\
\hline Z145 & G069 \\
\hline Z146 & G050 \\
\hline Z147 & G075 \\
\hline Z148 & G125 \\
\hline Z149 & G058 \\
\hline Z150 & G176 \\
\hline Z151 & G114 \\
\hline Z152 & G166 \\
\hline Z153 & G081 \\
\hline Z154 & G160 \\
\hline Z155 & G036 \\
\hline Z156 & \\
\hline Z157 & \\
\hline$Z 158$ & \\
\hline Z159 & \\
\hline Z160 & \\
\hline & \\
\hline
\end{tabular}

Comments

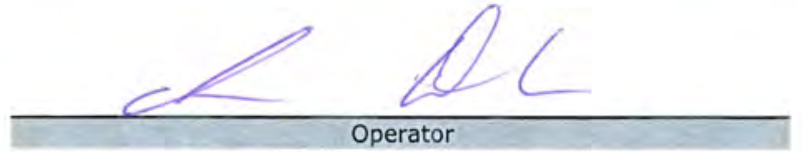

$$
4-15-09
$$


Data Report Form DRF-24D: Compact Charge Weight

\begin{tabular}{|c|c|}
\hline Procedure: & AGR-CHAR-DAM-24 Rev. 6 \\
\hline Operator: & Ivan Dunbar \\
\hline Compact lot ID: & LEU 07-OP1-Z \\
\hline Compact Lot description: & AGR-2 UCO Baseline, from G73J-14-93072A \\
\hline \multicolumn{2}{|c|}{ Filename: \IImc-agr|AGR\CompactDimensions\LEU07-OP1_ DRF24R6.xIs } \\
\hline \multicolumn{2}{|c|}{\begin{tabular}{|l|l|} 
Analytical balance calibration due date: & $10 / 29 / 09$ \\
\end{tabular}} \\
\hline \multicolumn{2}{|c|}{$\begin{array}{r}\text { Target compact charge weight }(\mathrm{g}): 6.4580 \\
\end{array}$} \\
\hline Average weight per overcoated particle $(\mathrm{g})$ : & $2.018 \mathrm{E}-03$ \\
\hline Approximate number of particles per compact: & 3200 \\
\hline Average uranium loading per particle $(\mathrm{g})$ : & $3.930 \mathrm{E}-04$ \\
\hline Approximate uranium loading per compact $(\mathrm{g})$ : & 1.258 \\
\hline
\end{tabular}

\begin{tabular}{|c|c|}
\hline $\begin{array}{c}\text { Compact } \\
\text { G Number }\end{array}$ & $\begin{array}{c}\text { Charge Weight } \\
\text { (9) }\end{array}$ \\
\hline G001 & 6.4587 \\
\hline G002 & 6.4581 \\
\hline G003 & 6.4573 \\
\hline G004 & 6.4591 \\
\hline G005 & 6.4570 \\
\hline G006 & 6.4587 \\
\hline G007 & 6.4567 \\
\hline G008 & 6.4595 \\
\hline G009 & 6.4578 \\
\hline G010 & 6.4575 \\
\hline G011 & 6.4580 \\
\hline G012 & 6.4568 \\
\hline G013 & 6.4584 \\
\hline G014 & 6.4584 \\
\hline G015 & 6.4583 \\
\hline G016 & 6.4582 \\
\hline G017 & 6.4585 \\
\hline G018 & 6.4582 \\
\hline G019 & 6.4590 \\
\hline G020 & 6.4585 \\
\hline G021 & 6.4586 \\
\hline G022 & 6.4587 \\
\hline G023 & 6.4585 \\
\hline G024 & 6.4582 \\
\hline G025 & 6.4574 \\
\hline G026 & 6.4577 \\
\hline G027 & 6.4580 \\
\hline G028 & 6.4589 \\
\hline G029 & 6.4583 \\
\hline G030 & 6.4579 \\
\hline G031 & 6.4592 \\
\hline G033 & 6.4579 \\
\hline G034 & 6.4582 \\
\hline G035 & 6.4587 \\
\hline G036 & 6.4576 \\
\hline G037 037 & 6.4583 \\
\hline G040 & 6.4583 \\
\hline & 6.4575 \\
\hline 6.4580 \\
\hline
\end{tabular}

\begin{tabular}{|c|c|}
\hline $\begin{array}{l}\text { Compact } \\
\text { G Number }\end{array}$ & $\begin{array}{c}\text { Charge Weight } \\
\text { (g) }\end{array}$ \\
\hline G046 & 6.4595 \\
\hline G047 & 6.4583 \\
\hline G048 & 6.4583 \\
\hline G049 & 6.4584 \\
\hline G050 & 6.4576 \\
\hline G051 & 6.4570 \\
\hline G052 & 6.4582 \\
\hline G053 & 6.4576 \\
\hline G054 & 6.4587 \\
\hline G055 & 6.4594 \\
\hline G056 & 6.4589 \\
\hline G057 & 6.4581 \\
\hline G058 & 6.4588 \\
\hline G059 & 6.4593 \\
\hline G060 & 6.4575 \\
\hline G061 & 6.4592 \\
\hline G062 & 6.4580 \\
\hline G063 & 6.4573 \\
\hline G064 & 6.4570 \\
\hline G065 & 6.4572 \\
\hline G066 & 6.4579 \\
\hline G067 & 6.4569 \\
\hline G068 & 6.4593 \\
\hline G069 & 6.4593 \\
\hline G070 & 6.4582 \\
\hline G071 & 6.4579 \\
\hline G072 & 6.4583 \\
\hline G073 & 6.4579 \\
\hline G074 & 6.4594 \\
\hline G075 & 6.4579 \\
\hline G076 & 6.4591 \\
\hline G077 & 6.4587 \\
\hline G078 & 6.4572 \\
\hline G079 & 6.4592 \\
\hline G080 & 6.4593 \\
\hline G081 & 6.4589 \\
\hline G082 & 6.4583 \\
\hline G083 & 6.4580 \\
\hline G084 & 6.4579 \\
\hline G085 & 6.4573 \\
\hline G086 & 6.4591 \\
\hline G087 & 6.4587 \\
\hline G088 & 6.4592 \\
\hline G089 & 6.4590 \\
\hline G090 & 6.4588 \\
\hline
\end{tabular}

\begin{tabular}{|c|c|}
\hline $\begin{array}{c}\text { Compact } \\
\text { G Number }\end{array}$ & $\begin{array}{c}\text { Charge Weight } \\
(\mathrm{g})\end{array}$ \\
\hline G091 & 6.4594 \\
\hline G092 & 6.4572 \\
\hline G093 & 6.4582 \\
\hline G094 & 6.4583 \\
\hline G095 & 6.4579 \\
\hline G096 & 6.4585 \\
\hline G097 & 6.4580 \\
\hline G098 & 6.4581 \\
\hline G099 & 6.4581 \\
\hline G100 & 6.4593 \\
\hline G101 & 6.4583 \\
\hline G102 & 6.4576 \\
\hline G103 & 6.4577 \\
\hline G104 & 6.4591 \\
\hline G105 & 6.4574 \\
\hline G106 & 6.4586 \\
\hline G107 & 6.4592 \\
\hline G108 & 6.4586 \\
\hline G109 & 6.4587 \\
\hline G110 & 6.4587 \\
\hline G111 & 6.4583 \\
\hline G112 & 6.4570 \\
\hline G113 & 6.4578 \\
\hline G114 & 6.4579 \\
\hline G115 & 6.4583 \\
\hline G116 & 6.4590 \\
\hline G117 & 6.4583 \\
\hline G118 & 6.4596 \\
\hline G119 & 6.4572 \\
\hline G120 & 6.4574 \\
\hline G121 & 6.4589 \\
\hline G122 & 6.4581 \\
\hline G123 & 6.4577 \\
\hline G124 & 6.4579 \\
\hline G125 & 6.4587 \\
\hline G126 & 6.4582 \\
\hline G128 & 6.4587 \\
\hline G129 & 6.45730 \\
\hline G131 & 6.4568 \\
\hline & 6.4575 \\
\hline G135 & 6.4577 \\
\hline & 6.4577 \\
\hline & 6.4581 \\
\hline
\end{tabular}

\begin{tabular}{|c|c|}
\hline $\begin{array}{c}\text { Compact } \\
\text { G Number }\end{array}$ & $\begin{array}{c}\text { Charge Weight } \\
(\text { ( ) }\end{array}$ \\
\hline G136 & 6.4582 \\
\hline G137 & 6.4585 \\
\hline G138 & 6.4572 \\
\hline G139 & 6.4561 \\
\hline G140 & 6.4599 \\
\hline G141 & 6.4571 \\
\hline G142 & 6.4588 \\
\hline G143 & 6.4576 \\
\hline G144 & 6.4585 \\
\hline G145 & 6.4589 \\
\hline G146 & 6.4591 \\
\hline G147 & 6.4589 \\
\hline G148 & 6.4594 \\
\hline G149 & 6.4590 \\
\hline G150 & 6.4593 \\
\hline G151 & 6.4581 \\
\hline G152 & 6.4585 \\
\hline G153 & 6.4592 \\
\hline G154 & 6.4579 \\
\hline G155 & 6.4585 \\
\hline G156 & 6.4582 \\
\hline G157 & 6.4564 \\
\hline G158 & 6.4575 \\
\hline G159 & 6.4581 \\
\hline G160 & 6.4583 \\
\hline G161 & 6.4568 \\
\hline G162 & 6.4577 \\
\hline G163 & 6.4575 \\
\hline G164 & 6.4569 \\
\hline G165 & 6.4574 \\
\hline G166 & 6.4573 \\
\hline G167 & 6.4586 \\
\hline G168 & 6.4577 \\
\hline G169 & 6.4584 \\
\hline G170 & 6.4568 \\
\hline G171 & 6.4597 \\
\hline G173 & 6.4593 \\
\hline G174 & 6.4580 \\
\hline G176 & 6.4580 \\
\hline & 6.4583 \\
\hline & 6.4576 \\
\hline G177 & 6.4575 \\
\hline G.4579 \\
\hline G.4572 \\
\hline
\end{tabular}

Comments

$$
\frac{4-1-09}{\text { Date }}
$$


Data Report Form DRF-25: Fuel Compact Mean Uranium Loading

\begin{tabular}{|r|l|}
\hline Procedure: & AGR-CHAR-DAM-25 Rev. 2 \\
\hline Operator: & Montgomery \\
\hline Compact lot ID: & LEU07-OP1-Z \\
\hline Compact lot description: & AGR-2 UCO Baseline fuel, from G73J-14-93072A \\
\hline Filename: & IIImC-agrIAGRIUraniumLoadinglLEU07-OP1-Z_DRF25R2.xIs \\
\hline
\end{tabular}

\begin{tabular}{|c|c|c|c|c|c|c|}
\hline & Sample 1 & Sample 2 & Sample 3 & Sample 4 & Sample 5 & Sample 6 \\
\hline Compact ID number: & Z057 & Z154 & $Z 096$ & Z090 & $\mathrm{Z} 120$ & $\mathrm{Z153}$ \\
\hline First Leach & \multicolumn{6}{|c|}{ 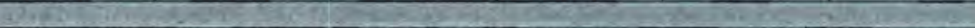 } \\
\hline Sample tube ID number: & U09042001 & U09042002 & U09042003 & U09042004 & U09042005 & U09042006 \\
\hline Radiochemical laboratory analysis number: & $1874-001$ & $1874-002$ & $1874-003$ & $1874-004$ & $1874-005$ & $1874-006$ \\
\hline Weight $U$ in leach $(g)$ : & 1.251 & 1.255 & 1.242 & 1.253 & 1.250 & 1.254 \\
\hline Uncertainty in weight $U$ in leach $(\mathrm{g})$ : & 0.005 & 0.005 & 0.005 & 0.005 & 0.005 & 0.005 \\
\hline \multicolumn{7}{|l|}{ Second Leach } \\
\hline Sample tube ID number: & U09042101 & U09042102 & U09042103 & U09042104 & U09042105 & U09042106 \\
\hline Radiochemical laboratory analysis number: & $1874-007$ & $1874-008$ & $1874-009$ & $1874-010$ & $1874-011$ & $1874-012$ \\
\hline Weight $U$ in leach $(g)$ : & $3.05 \mathrm{E}-04$ & $2.88 \mathrm{E}-04$ & $4.13 E-04$ & $2.94 \mathrm{E}-04$ & $4.86 \mathrm{E}-04$ & $3.80 \mathrm{E}-04$ \\
\hline Uncertainty in weight $U$ in leach $(q)$ : & $3.05 \mathrm{E}-05$ & $2.88 \mathrm{E}-05$ & $4.13 E-05$ & $2.94 \mathrm{E}-05$ & $4.86 \mathrm{E}-05$ & $3.80 \mathrm{E}-05$ \\
\hline Total Measured U & & $=$ & $\sqrt{2+2 x}$ & +2 & & \\
\hline Weight $U$ in compact $(\mathrm{g})$ : & 1.251 & 1.255 & 1.242 & 1.253 & 1.251 & 1.254 \\
\hline Uncertainty in weight $\mathrm{U}$ in compact (g): & 0.005 & 0.005 & 0.005 & 0.005 & 0.005 & 0.005 \\
\hline \multicolumn{3}{|c|}{ Mean uranium loading (gU/compact): } & \multirow{2}{*}{\multicolumn{3}{|c|}{$\frac{1.251}{0.005}$}} & \\
\hline \multicolumn{3}{|c|}{ Standard deviation in mean uranium loading (gU/compact): } & & & & \\
\hline
\end{tabular}

Comments

each 1 was analyzed by Davies-Gray titration method. Leach 2 was analyzed by ICP-MS, due to low U concentration.

Davies-Gray Initial known U recovery: $100.25 \%$; final known U recovery $100.18 \%$ Blind titration U recovery $100.39 \%$.

Uncertainty in Davies-Gray $(0.4 \%)$ based on average of measured \% recovery data for LEU06,07,08,09.

wt. \% U235 enrichment: sample $1=14.542$ : sample $2=14.529 ;$ sample $3=14.533$; sample $4=14.529 ;$ sample $5=14.501$; sample $6=14.504$

$U$ data check with official results of analysies for RMAL 1874 by FCM on 5/26/2009

Feed c. montgomery $10-19-2009$

Operator 
Data Report Form DRF-26A: Measurement of U Contamination or Impurities by Deconsolidation Leach

\begin{tabular}{|c|c|}
\hline Procedure: & AGR-CHAR-DAM-26 ReV. 1 \\
\hline Operator: & Fred Montgomery \\
\hline Compact lot ID: & LLU07-OP1-Z \\
\hline $\begin{array}{l}\text { Compact lot description: } \\
\text { Compact ID numbers: }\end{array}$ & $\frac{\text { AGR-2 UCO Baseline Fuel, from G73J-14-93072A }}{060,046,122,146,130}$ \\
\hline DRF filename: & 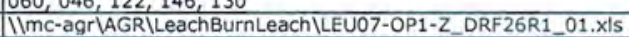 \\
\hline
\end{tabular}

\begin{tabular}{|c|c|c|c|c|}
\hline \multicolumn{2}{|r|}{$\begin{array}{r}\text { Mean average weight uranium per particle }(g): \\
\text { Uncertainty in mean average weight uranium per particle }(\mathrm{q}):\end{array}$} & \multicolumn{3}{|c|}{$\begin{array}{c}3.930 \mathrm{E}-04 \\
6.56 \mathrm{E}-07 \\
\end{array}$} \\
\hline & & First Leach & Second Leach & Total \\
\hline & Deconsolidation-leach solution ID: & $L 09042401$ & $L 09042801$ & \\
\hline & Number of compacts: & & & \\
\hline & Total volume of leach solution $(\mathrm{ml})$ : & 145.0 & 140.0 & \\
\hline & Radiochemical laboratory analysis number: | & $1881-001$ & & \\
\hline & Measured uranium concentration $(\mathrm{\mu g} / \mathrm{ml})$ : & $2.82 \mathrm{E}+00$ & $1.47 \mathrm{E}-01$ & \\
\hline & Uncertainty in uranium concentration $(\mu \mathrm{g} / \mathrm{ml})$ : & $2.82 \mathrm{E}-01$ & $1.47 \mathrm{E}-02$ & \\
\hline & Weight uranium leached $(g):$ & $4.09 \mathrm{E}-04$ & $2.06 \mathrm{E}-05$ & $4.29 \mathrm{E}-04$ \\
\hline & Uncertainty in weight uranium leached $(9):$ & $4.09 \mathrm{E}-05$ & $2.06 \mathrm{E}-06$ & $4.10 \mathrm{E}-05$ \\
\hline & Effective number of exposed kernels: & 1.0 & 0.1 & 1.1 \\
\hline & Uncertainty in effective number of exposed kernels: & 0.1 & 0.0 & 0.1 \\
\hline \multirow{5}{*}{$\mathbf{F e}$} & Measured concentration of impurity in sample $(\mathrm{\mu g} / \mathrm{ml})$ : & $<1.03 \mathrm{E}-01$ & $<1.03 \mathrm{E}-01$ & \\
\hline & Uncorrected weight of impurity in sample $(\mu g)$ : & $<14.94$ & $<14.42$ & $<29.36$ \\
\hline & Weight of impurity in blank $(\mu \mathrm{g})$ : & $<18.33$ & $<14.11$ & \\
\hline & Minimum corrected weight of impurity in sample $(\mu \mathrm{g})$ : & 0.00 & 0.00 & 0.00 \\
\hline & Maximum corrected weight of impurity in sample $(\mu g)$ : & 14.94 & 14.42 & 29.36 \\
\hline \multirow{5}{*}{$\mathrm{Cr}$} & Measured concentration of impurity in sample $(\mathrm{\mu g} / \mathrm{ml})$ : & $7.08 E-03$ & $5.47 \mathrm{E}-03$ & $\mathrm{Cr}$ \\
\hline & Uncorrected weight of impurity in sample $(\mu g)$ : & 1.03 & 0.77 & 1.79 \\
\hline & Weight of impurity in blank $(\mu g)$ : & $<0.89$ & $<0.69$ & \\
\hline & Minimum corrected weight of impurity in sample $(\mu \mathrm{g})$ : & 0.14 & 0.08 & 0.22 \\
\hline & Maximum corrected weight of impurity in sample $(\mu g)$ : & 1.03 & 0.77 & 1.79 \\
\hline \multirow{5}{*}{$\mathrm{Mn}$} & Measured concentration of impurity in sample $(\mathrm{\mu g} / \mathrm{ml})$ : & $<4.77 \mathrm{E}-03$ & $\leq 4.77 \mathrm{E}-03$ & $\mathrm{Mn}$ \\
\hline & Uncorrected weight of impurity in sample $(\mu g)$ : & $<0.69$ & $<0.67$ & $<1,36$ \\
\hline & Weight of impurity in blank $(\mu \mathrm{g})$ : & $<0.85$ & $<0.65$ & \\
\hline & Minimum corrected weight of impurity in sample $(\mu g)$ : & 0.00 & 0.00 & 0.00 \\
\hline & Maximum corrected weight of impurity in sample $(\mu g)$ : & 0.69 & 0.67 & 1.36 \\
\hline \multirow{5}{*}{ co } & Measured concentration of impurity in sample $(\mu \mathrm{g} / \mathrm{ml})$ : & $<4.06 \mathrm{E}-03$ & $<4.06 \mathrm{E}-03$ & Co \\
\hline & Uncorrected weight of impurity in sample $(\mu \mathrm{g})$ : & $<0.59$ & $<0.57$ & $<1.16$ \\
\hline & Weight of impurity in blank $(\mu \mathrm{g})$ : & $<0.72$ & $<0.56$ & \\
\hline & Minimum corrected weight of impurity in sample $(\mu \mathrm{g})$ : & 0.00 & 0.00 & 0.00 \\
\hline & Maximum corrected weight of impurity in sample $(\mu \mathrm{g})$ : & 0.59 & 0.57 & 1.16 \\
\hline \multirow{5}{*}{$\mathrm{Ni}$} & Measured concentration of impurity in sample $(\mathrm{\mu g} / \mathrm{ml})$ : & $<2.00 \mathrm{E}-02$ & $<2.00 \mathrm{E}-02$ & $\mathrm{Ni}$ \\
\hline & Uncorrected weight of impurity in sample $(\mu g)$ : & $<2.90$ & $<2.80$ & $<5.70$ \\
\hline & Weight of impurity in blank $(\mu \mathrm{g})$ : & $<3.56$ & $<2.74$ & \\
\hline & Minimum corrected weight of impurity in sample $(\mu g)$ : & 0.00 & 0.00 & 0.00 \\
\hline & Maximum corrected weight of impurity in sample $(\mu q)$ : & 2.90 & 2.80 & 5.70 \\
\hline \multirow{5}{*}{$\mathrm{Ca}$} & Measured concentration of impurity in sample $(\mu \mathrm{g} / \mathrm{ml})$ : & $4.63 \mathrm{E}-01$ & 2.16E-01 & $\mathrm{Ca}$ \\
\hline & Uncorrected weight of impurity in sample $(\mu \mathrm{g})$ : & 67.14 & 30.24 & 97.38 \\
\hline & Weight of impurity in blank $(\mu \mathrm{g})$ : & $<17.80$ & 42.61 & \\
\hline & Minimum corrected weight of impurity in sample $(\mu \mathrm{g})$ : & 49.34 & 0.00 & 49.34 \\
\hline & Maximum corrected weight of impurity in sample $(\mu g)$ : & 67.14 & 0.00 & 67.14 \\
\hline \multirow{5}{*}{ Al } & Measured concentration of impurity in sample $(\mu \mathrm{g} / \mathrm{ml})$ : & $4.32 \mathrm{E}-01$ & $1.50 \mathrm{E}-01$ & Al \\
\hline & Uncorrected weight of impurity in sample $(\mu g)$ : & 62.64 & 21.00 & 83.64 \\
\hline & Weight of impurity in blank $(\mu \mathrm{g})$ : & 5.46 & 3.45 & \\
\hline & Minimum corrected weight of impurity in sample $(\mu g)$ : & 57.18 & 17.55 & 74.72 \\
\hline & Maximum corrected weight of impurity in sample $(\mu q)$ : & 57.18 & 17.55 & 74.72 \\
\hline \multirow{5}{*}{$\mathrm{Ti}$} & Measured concentration of impurity in sample $(\mu \mathrm{g} / \mathrm{ml})$ : & $2.06 \mathrm{E}-02$ & $2.19 \mathrm{E}-02$ & $\mathrm{Ti}$ \\
\hline & Uncorrected weight of impurity in sample $(\mu q)$ : & 2.99 & 3.07 & 6.05 \\
\hline & Weight of impurity in blank $(\mu \mathrm{g})$ : & $<3.56$ & $<2.74$ & \\
\hline & Minimum corrected weight of impurity in sample $(\mu g)$ : & 0.00 & 0.33 & 0.33 \\
\hline & Maximum corrected weight of impurity in sample $(\mu g)$ : & 2.99 & 3.07 & 6.05 \\
\hline \multirow{5}{*}{ v } & Measured concentration of impurity in sample $(\mu \mathrm{g} / \mathrm{ml})$ : & $2.22 \mathrm{E}-01$ & $5.76 \mathrm{E}-02$ & $\mathrm{v}$ \\
\hline & Uncorrected weight of impurity in sample $(\mu \mathrm{g})$ : & 32.19 & 8.06 & 40.25 \\
\hline & Weight of impurity in blank $(\mu g)$ : & $<0.89$ & $<0.69$ & +2 \\
\hline & Minimum corrected weight of impurity in sample $(\mu g)$ : & 31.30 & 7.38 & 38.68 \\
\hline & Maximum corrected weight of impurity in sample $(\mu q)$ : & 32.19 & 8.06 & 40.25 \\
\hline
\end{tabular}

Comments

FCM Checked the official results of analyses for RMAL1881 on 7/10/2009

Fied C. montgo mevey

$7-23-09$ 
Data Report Form DRF-26A: Measurement of U Contamination or Impurities by Deconsolidation Leach

\begin{tabular}{|c|c|}
\hline Procedure: & AGR-CHAR-DAM-26 Rev. 1 \\
\hline Operator: & Fred Montgomery \\
\hline Compact lot ID: & LEU07-OP1-Z \\
\hline Compact lot description: & AGR-2 UCO Baseline Fuel, from G73J-14-93072A \\
\hline Compact ID numbers: & $141,138,111,064,053$ \\
\hline DRF filename: & IIImc-agr|AGRILeachBurnLeach|LEU07-OP1-Z_DRF26R1_01.xIs \\
\hline
\end{tabular}

\begin{tabular}{|c|c|c|c|c|}
\hline \multirow{2}{*}{\multicolumn{2}{|c|}{$\begin{array}{r}\text { Mean average weight uranium per particle }(g) \text { : } \\
\text { Uncertainty in mean average weight uranium per particle }(q):\end{array}$}} & \multirow{2}{*}{\multicolumn{3}{|c|}{$\frac{3.93 \mathrm{E}-04}{6.56 \mathrm{E}-07}$}} \\
\hline & & & & \\
\hline & & First Leach & Second Leach & Total \\
\hline \multirow{2}{*}{\multicolumn{2}{|c|}{$\begin{array}{r}\text { Deconsolidation-leach solution ID: } \\
\text { Number of compacts: }\end{array}$}} & L09042402 & L09042802 & \\
\hline & & & & \\
\hline \multicolumn{2}{|r|}{ Total volume of leach solution $(\mathrm{ml})$ : } & 148.0 & 141.0 & \\
\hline & 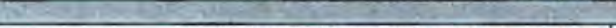 & 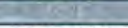 & & \\
\hline \multicolumn{2}{|r|}{ Radiochemical laboratory analysis number: } & 1881.002 & $1881-009$ & \\
\hline \multicolumn{2}{|r|}{ Measured uranium concentration $(\mu \mathrm{g} / \mathrm{ml})$ : } & $2.83 E+00$ & $1.25 \mathrm{E}-01$ & \\
\hline & Uncertainty in uranium concentration $(\mu \mathrm{g} / \mathrm{ml})$ : & $2.83 E-01$ & $1.25 \mathrm{E}-02$ & \\
\hline \multicolumn{2}{|r|}{ Weight uranium leached $(\mathrm{g})$ : } & $4.19 \mathrm{E}-04$ & 1.76E-05 & $4.36 \mathrm{E}-04$ \\
\hline \multicolumn{2}{|r|}{ Uncertainty in weight uranium leached $(\mathrm{g})$ : } & $4.19 \mathrm{E}-05$ & $1.76 \mathrm{E}-06$ & $4.20 E-05$ \\
\hline \multirow{2}{*}{\multicolumn{2}{|c|}{$\begin{array}{r}\text { Effective number of exposed kernels: } \\
\text { Uncertainty in effective number of exposed kernels: }\end{array}$}} & 1.1 & 0.0 & 1.1 \\
\hline & & 0.1 & 0.0 & 0.1 \\
\hline & & & & \\
\hline \multirow{5}{*}{$\mathrm{Fe}$} & Measured concentration of impurity in sample $(\mathrm{\mu g} / \mathrm{ml})$ : & $<1.03 \mathrm{E}-01$ & $<1.03 \mathrm{E}-01$ & $\mathrm{Fe}$ \\
\hline & Uncorrected weight of impurity in sample $(\mu g)$ : & $<15.24$ & $<14.52$ & $<29.77$ \\
\hline & Weight of impurity in blank $(\mu \mathrm{g})$ : & $<18.33$ & $<14.11$ & $=$ \\
\hline & Minimum corrected weight of impurity in sample $(\mu \mathrm{g})$ : & 0.00 & 0.00 & 0.00 \\
\hline & Maximum corrected weight of impurity in sample $(\mu \mathrm{g})$ : & 15.24 & 14.52 & 29.77 \\
\hline \multirow{5}{*}{$\mathrm{Cr}$} & Measured concentration of impurity in sample $(\mu \mathrm{g} / \mathrm{ml})$ : & $8.93 E-03$ & $<5.00 \mathrm{E}-03$ & $\mathrm{Cr}$ \\
\hline & Uncorrected weight of impurity in sample $(\mu \mathrm{g})$ : & 1.32 & $<0.71$ & $<2.03$ \\
\hline & Weight of impurity in blank $(\mu \mathrm{g})$ : & $<0.89$ & $<0.69$ & 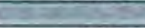 \\
\hline & Minimum corrected weight of impurity in sample $(\mu \mathrm{g})$ : & 0.43 & 0.00 & 0.43 \\
\hline & Maximum corrected weight of impurity in sample $(\mu \mathrm{g})$ : & 1.32 & 0.71 & 2.03 \\
\hline \multirow{5}{*}{ Mn } & Measured concentration of impurity in sample $(\mu \mathrm{g} / \mathrm{ml})$ : & $<4.77 \mathrm{E}-03$ & $<4.77 \mathrm{E}-03$ & Mn \\
\hline & Uncorrected weight of impurity in sample $(\mu \mathrm{g})$ : & $<0.71$ & $<0.67$ & $<1.38$ \\
\hline & Weight of impurity in blank $(\mu \mathrm{g})$ : & $<0.85$ & $<0.65$ & 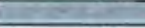 \\
\hline & Minimum corrected weight of impurity in sample $(\mu g)$ : & 0.00 & 0.00 & 0.00 \\
\hline & Maximum corrected weight of impurity in sample $(\mu \mathrm{g})$ : & 0.71 & 0.67 & 1.38 \\
\hline \multirow{5}{*}{ Co } & Measured concentration of impurity in sample $(\mu \mathrm{g} / \mathrm{ml})$ : & $<4.06 \mathrm{E}-03$ & $<4.06 \mathrm{E}-03$ & Co \\
\hline & Uncorrected weight of impurity in sample $(\mu \mathrm{g})$ : & $<0.60$ & $<0.57$ & $<1.17$ \\
\hline & Weight of impurity in blank $(\mu \mathrm{g})$ : & $<0.72$ & $<0.56$ & 8 \\
\hline & Minimum corrected weight of impurity in sample $(\mu \mathrm{g})$ : & 0.00 & 0.00 & 0.00 \\
\hline & Maximum corrected weight of impurity in sample $(\mu \mathrm{g})$ : & 0.60 & 0.57 & 1.17 \\
\hline \multirow{5}{*}{$\mathrm{Ni}$} & Measured concentration of impurity in sample $(\mu \mathrm{g} / \mathrm{ml})$ : & $<2.00 \mathrm{E}-02$ & $<2.00 \mathrm{E}-02$ & $\mathrm{Ni}$ \\
\hline & Uncorrected weight of impurity in sample $(\mu \mathrm{g})$ : & $<2.96$ & $<2.82$ & $<5.78$ \\
\hline & Weight of impurity in blank $(\mu \mathrm{g})$ : & $<3.56$ & $<2.74$ & \\
\hline & Minimum corrected weight of impurity in sample $(\mu g)$ : & 0.00 & 0.00 & 0.00 \\
\hline & Maximum corrected weight of impurity in sample $(\mu g)$ : & 2.96 & 2.82 & 5.78 \\
\hline \multirow{5}{*}{ Ca } & Measured concentration of impurity in sample $(\mu \mathrm{g} / \mathrm{ml})$ : & $5.02 \mathrm{E}-01$ & $1.31 \mathrm{E}-01$ & $\mathrm{Ca}$ \\
\hline & Uncorrected weight of impurity in sample $(\mu \mathrm{g})$ : & 74.30 & 18.47 & 92.77 \\
\hline & Weight of impurity in blank $(\mu g)$ : & $<17.80$ & 42.61 & \\
\hline & Minimum corrected weight of impurity in sample $(\mu \mathrm{g})$ : & 56.50 & 0.00 & 56.50 \\
\hline & Maximum corrected weight of impurity in sample $(\mu g)$ : & 74.30 & 0.00 & 74.30 \\
\hline \multirow{5}{*}{ Al } & Measured concentration of impurity in sample $(\mathrm{\mu g} / \mathrm{ml})$ : & $5.28 \mathrm{E}-01$ & $1.07 \mathrm{E}-01$ & Al \\
\hline & Uncorrected weight of impurity in sample $(\mu \mathrm{g})$ : & 78.14 & 15.09 & 93.23 \\
\hline & Weight of impurity in blank $(\mu \mathrm{g})$ : & 5.46 & 3.45 & 5 \\
\hline & Minimum corrected weight of impurity in sample $(\mu g)$ : & 72.68 & 11.63 & 84.31 \\
\hline & Maximum corrected weight of impurity in sample $(\mu \mathrm{g})$ : & 72.68 & 11.63 & 84.31 \\
\hline \multirow{5}{*}{$\mathrm{Ti}$} & Measured concentration of impurity in sample $(\mu \mathrm{g} / \mathrm{ml})$ : & $3.26 \mathrm{E}-02$ & 2.05E-02 & $\mathrm{Ti}$ \\
\hline & Uncorrected weight of impurity in sample $(\mu \mathrm{g})$ : & 4.82 & 2.89 & 7.72 \\
\hline & Weight of impurity in blank $(\mu \mathrm{g})$ : & $<3.56$ & $<2.74$ & $\mathrm{C}=\mathrm{n}$ \\
\hline & Minimum corrected weight of impurity in sample $(\mu \mathrm{g})$ : & 1.26 & 0.15 & 1.42 \\
\hline & Maximum corrected weight of impurity in sample $(\mu g)$ : & 4.82 & 2.89 & 7.72 \\
\hline & Measured concentration of impurity in sample $(\mu \mathrm{g} / \mathrm{ml})$ : & $2.22 \mathrm{E}-01$ & 5.10E-02 & v \\
\hline & Uncorrected weight of impurity in sample $(\mu \mathrm{g})$ : & 32.86 & 7.19 & 40.05 \\
\hline $\mathbf{v}$ & Weight of impurity in blank $(\mu \mathrm{g})$ : & $<0.89$ & $<0.69$ & 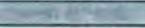 \\
\hline & Minimum corrected weight of impurity in sample $(\mu \mathrm{g})$ : & 31.97 & 6.51 & 38.47 \\
\hline & Maximum corrected weight of impurity in sample $(\mu \mathrm{g})$ : & 32.86 & 7.19 & 40.05 \\
\hline
\end{tabular}

comments

Fied c. Mnontgomeuy

$7-23-09$ 
Data Report Form DRF-26A: Measurement of U Contamination or Impurities by Deconsolidation Leach

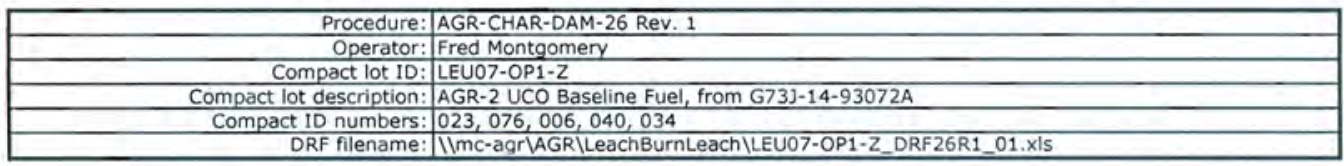

\begin{tabular}{|c|c|c|c|c|}
\hline \multicolumn{2}{|c|}{$\begin{array}{c}\text { Mean average weight uranium per particle }(g): \\
\text { Uncertainty in mean average weight uranium per particle }(q):\end{array}$} & \multicolumn{3}{|c|}{$\frac{3.93 E-04}{6.56 E-07}$} \\
\hline & & First Leach & Second Leach & Total \\
\hline & Deconsolidation-leach solution ID: & $\angle 09042403$ & $L 09042803$ & \\
\hline & Number of compacts: & & & \\
\hline & Total volume of leach solution $(\mathrm{ml})$ : & 147.0 & 139.0 & \\
\hline & Radiochemical laboratory analysis number: & $1881-003$ & $1881-010$ & \\
\hline & Measured uranium concentration $(\mathrm{\mu g} / \mathrm{ml})$ : & $4.82 \mathrm{E}-02$ & $9.97 \mathrm{E}-03$ & \\
\hline & Uncertainty in uranium concentration $(\mu \mathrm{g} / \mathrm{ml})$ : & $4.82 \mathrm{E}-03$ & $9.97 E-04$ & \\
\hline & Weight uranium leached $(\mathrm{g})$ : & $7.09 \mathrm{E}-06$ & $1.39 \mathrm{E}-06$ & $8.47 E-06$ \\
\hline & Uncertainty in weight uranium leached $(\mathrm{g})$ : & $7.09 \mathrm{E}-07$ & $1.39 \mathrm{E}-07$ & $7.23 E-07$ \\
\hline & Effective number of exposed kernels: & 0.0 & 0.0 & 0.0 \\
\hline & Uncertainty in effective number of exposed kernels: & 0.0 & 0.0 & 0.0 \\
\hline \multirow{5}{*}{$\mathrm{Fe}$} & Measured concentration of impurity in sample $(\mu \mathrm{g} / \mathrm{ml})$ : & $<1.03 \mathrm{E}-01$ & $<1.03 E-01$ & $\mathbf{F e}$ \\
\hline & Uncorrected weight of impurity in sample $(\mu g)$ : & $<15.14$ & $<14.32$ & $<29.46$ \\
\hline & Weight of impurity in blank $(\mu \mathrm{g})$ : & $<18.33$ & $<14.11$ & \\
\hline & Minimum corrected weight of impurity in sample $(\mu g)$ : & 0.00 & 0.00 & 0.00 \\
\hline & Maximum corrected weight of impurity in sample $(\mu g)$ : & 15.14 & 14.32 & 29.46 \\
\hline \multirow{5}{*}{$\mathrm{Cr}$} & Measured concentration of impurity in sample $(\mu \mathrm{g} / \mathrm{ml})$ : & $1.18 \mathrm{E}-02$ & $<5.00 \mathrm{E}-03$ & $\mathrm{Cr}$ \\
\hline & Uncorrected weight of impurity in sample $(\mu g)$ : & 1.73 & $<0.70$ & $<2.43$ \\
\hline & Weight of impurity in blank $(\mu \mathrm{g})$ : & $<0.89$ & $<0.69$ & \\
\hline & Minimum corrected weight of impurity in sample $(\mu q)$ : & 0.84 & 0.00 & 0.84 \\
\hline & Maximum corrected weight of impurity in sample $(\mu g)$ : & 1.73 & 0.70 & 2.43 \\
\hline \multirow{5}{*}{ Mn } & Measured concentration of impurity in sample $(\mathrm{\mu g} / \mathrm{ml})$ : & $<4.77 \mathrm{E}-03$ & $\leq 4.77 \mathrm{E}-03$ & $\mathrm{Mn}$ \\
\hline & Uncorrected weight of impurity in sample $(\mu \mathrm{g})$ : & $<0.70$ & $<0.66$ & $<1.36$ \\
\hline & Weight of impurity in blank $(\mu \mathrm{g})$ : & $<0.85$ & $<0.65$ & \\
\hline & Minimum corrected weight of impurity in sample $(\mu g)$ : & 0.00 & 0.00 & 0.00 \\
\hline & Maximum corrected weight of impurity in sample $(\mu g)$ : & 0.70 & 0.66 & 1.36 \\
\hline \multirow{5}{*}{ co } & Measured concentration of impurity in sample $(\mathrm{\mu g} / \mathrm{ml})$ : & $<4.06 \mathrm{E}-03$ & $<4.06 \mathrm{E}-03$ & Co \\
\hline & Uncorrected weight of impurity in sample $(\mu g)$ : & $<0.60$ & $<0.56$ & $<1.16$ \\
\hline & Weight of impurity in blank $(\mu \mathrm{g})$ : & $<0.72$ & $<0.56$ & \\
\hline & Minimum corrected weight of impurity in sample $(\mu g)$ : & 0.00 & 0.00 & 0.00 \\
\hline & Maximum corrected weight of impurity in sample $(\mu \mathrm{g})$ : & 0.60 & 0.56 & 1.16 \\
\hline \multirow{5}{*}{$\mathrm{Ni}$} & Measured concentration of impurity in sample $(\mathrm{\mu g} / \mathrm{ml})$ : & $<2.00 \mathrm{E}-02$ & $<2.00 \mathrm{E}-02$ & $\mathrm{Ni}$ \\
\hline & Uncorrected weight of impurity in sample $(\mu g)$ : & $<2.94$ & $<2.78$ & $<5.72$ \\
\hline & Weight of impurity in blank $(\mu \mathrm{g})$ : & $<3.56$ & $<2.74$ & \\
\hline & Minimum corrected weight of impurity in sample $(\mu g)$ : & 0.00 & 0.00 & 0.00 \\
\hline & Maximum corrected weight of impurity in sample $(\mu \mathrm{g})$ : & 2.94 & 2.78 & 5.72 \\
\hline \multirow{5}{*}{$\mathrm{Ca}$} & Measured concentration of impurity in sample $(\mathrm{\mu g} / \mathrm{ml})$ : & $8.46 \mathrm{E}-01$ & $1.08 \mathrm{E}-01$ & $\mathrm{Ca}$ \\
\hline & Uncorrected weight of impurity in sample $(\mu \mathrm{g})$ : & 124.36 & 15.01 & 139.37 \\
\hline & Weight of impurity in blank $(\mu g)$ : & $<17.80$ & 42.61 & \\
\hline & Minimum corrected weight of impurity in sample $(\mu \mathrm{g})$ : & 106.56 & 0.00 & 106.56 \\
\hline & Maximum corrected weight of impurity in sample $(\mu g)$ : & 124.36 & 0.00 & 124.36 \\
\hline \multirow{5}{*}{ Al } & Measured concentration of impurity in sample $(\mathrm{hg} / \mathrm{ml})$ : & $4.23 E-01$ & $7.50 E-02$ & Al \\
\hline & Uncorrected weight of impurity in sample $(\mu \mathrm{g})$ : & 62.18 & 10.43 & 72.61 \\
\hline & Weight of impurity in blank $(\mu \mathrm{g})$ : & 5.46 & 3.45 & \\
\hline & Minimum corrected weight of impurity in sample $(\mu g)$ : & 56.72 & 6.97 & 63.69 \\
\hline & Maximum corrected weight of impurity in sample $(\mu g)$ : & 56.72 & 6.97 & 63.69 \\
\hline \multirow{5}{*}{$\mathrm{Ti}$} & Measured concentration of impurity in sample $(\mu \mathrm{g} / \mathrm{ml})$ : & $3.40 \mathrm{E}-02$ & $<2.00 \mathrm{E}-02$ & $\mathrm{Ti}$ \\
\hline & Uncorrected weight of impurity in sample $(\mu \mathrm{g})$ : & 5.00 & $<2.78$ & $<7.78$ \\
\hline & Weight of impurity in blank $(\mu g)$ : & $<3.56$ & $<2.74$ & $=$ \\
\hline & Minimum corrected weight of impurity in sample $(\mu g)$ : & 1.44 & 0.00 & 1.44 \\
\hline & Maximum corrected weight of impurity in sample $(\mu g)$ : & 5.00 & 2.78 & 7.78 \\
\hline \multirow{5}{*}{$\mathrm{v}$} & Measured concentration of impurity in sample $(\mu \mathrm{g} / \mathrm{ml})$ : & 2.17E-01 & $4.11 \mathrm{E}-02$ & $\mathbf{v}$ \\
\hline & Uncorrected weight of impurity in sample $(\mu g)$ : & 31.90 & 5.71 & 37.61 \\
\hline & Weight of impurity in blank $(\mu \mathrm{g})$ : & $<0.89$ & $<0.69$ & \\
\hline & Minimum corrected weight of impurity in sample $(\mu g)$ : & 31.01 & 5.03 & 36.04 \\
\hline & Maximum corrected weight of impurity in sample $(\mu g)$ : & 31.90 & 5.71 & 37.61 \\
\hline
\end{tabular}

Comments

treel c. Montgomery 
Data Report Form DRF-26A: Measurement of U Contamination or Impurities by Deconsolidation Leach

\begin{tabular}{|c|c|}
\hline Procedure: & AGR-CHAR-DAM- 26 Rev. 1 \\
\hline Operator: & Fred Montgomery \\
\hline Compact lot ID: & LEU 07-OP1-Z \\
\hline Compact lot description: & AGR-2 UCO Baseline Fuel, from G73J-14-93072A \\
\hline Compact ID numbers: & $139,083,087,145,049$ \\
\hline DRF filename: & 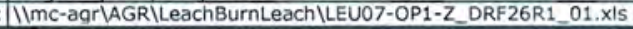 \\
\hline
\end{tabular}

\begin{tabular}{|c|c|c|c|c|}
\hline \multirow{2}{*}{\multicolumn{2}{|c|}{$\begin{array}{r}\text { Mean average weight uranium per particle }(g): \\
\text { Uncertainty in mean average weight uranium per particle }(q):\end{array}$}} & \multicolumn{3}{|c|}{$3.93 E-04$} \\
\hline & & \multicolumn{3}{|c|}{$6.56 \mathrm{E}-07$} \\
\hline & & First Leach & Second Leach & Total \\
\hline & Deconsolidation-leach solution ID: & $\mathrm{L} 09042404$ & $L 09042804$ & \\
\hline & Number of compacts: & \multicolumn{2}{|c|}{5} & \\
\hline & Total volume of leach solution $(\mathrm{ml})$ : & 147.0 & 138.0 & \\
\hline \multicolumn{4}{|c|}{ 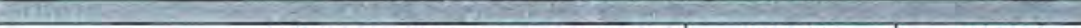 } & \\
\hline & Radiochemical laboratory analysis number: & $1881-004$ & $1881-011$ & \\
\hline & Measured uranium concentration $(\mu \mathrm{g} / \mathrm{ml})$ : & $1.36 \mathrm{E}+00$ & $1.27 E-01$ & \\
\hline & Uncertainty in uranium concentration $(\mu \mathrm{g} / \mathrm{ml})$ : & $1.36 \mathrm{E}-01$ & $1.27 \mathrm{E}-02$ & \\
\hline & Weight uranium leached $(\mathrm{g}):$ & $2.00 \mathrm{E}-04$ & $1.75 \mathrm{E}-05$ & $2.17 E-04$ \\
\hline & Uncertainty in weight uranium leached $(g)$ : & $2.00 \mathrm{E}-05$ & $1.75 \mathrm{E}-06$ & $2.01 \mathrm{E}-05$ \\
\hline & Effective number of exposed kernels: & 0.5 & 0.0 & 0.6 \\
\hline & Uncertainty in effective number of exposed kernels: & 0.1 & 0.0 & 0.1 \\
\hline & 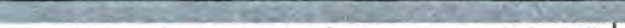 & & & \\
\hline \multirow{5}{*}{$\mathbf{F e}$} & Measured concentration of impurity in sample $(\mu \mathrm{g} / \mathrm{ml})$ : & $<1.03 \mathrm{E}-01$ & $<1.03 \mathrm{E}-01$ & $\mathrm{Fe}$ \\
\hline & Uncorrected weight of impurity in sample $(\mu \mathrm{g})$ : & $<15.14$ & $<14.21$ & $<29.36$ \\
\hline & Weight of impurity in blank $(\mu g)$ : & $<18.33$ & $<14.11$ & \\
\hline & Minimum corrected weight of impurity in sample $(\mu \mathrm{g})$ : & 0.00 & 0.00 & 0.00 \\
\hline & Maximum corrected weight of impurity in sample $(\mu \mathrm{g})$ : & 15.14 & 14.21 & 29.36 \\
\hline \multirow{5}{*}{$\mathrm{Cr}$} & Measured concentration of impurity in sample $(\mu \mathrm{g} / \mathrm{ml})$ : & $1.01 \mathrm{E}-02$ & $<5.00 \mathrm{E}-03$ & $\mathrm{Cr}$ \\
\hline & Uncorrected weight of impurity in sample $(\mu g)$ : & 1.48 & $<0.69$ & $<2.17$ \\
\hline & Weight of impurity in blank $(\mu g)$ : & $<0.89$ & $<0.69$ & 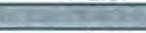 \\
\hline & Minimum corrected weight of impurity in sample $(\mu \mathrm{g})$ : & 0.59 & 0.00 & 0.59 \\
\hline & Maximum corrected weight of impurity in sample $(\mu g)$ : & 1.48 & 0.69 & 2.17 \\
\hline \multirow{5}{*}{ Mn } & Measured concentration of impurity in sample $(\mu \mathrm{g} / \mathrm{ml})$ : & $4.77 \mathrm{E}-03$ & $<4.77 \mathrm{E}-03$ & Mn \\
\hline & Uncorrected weight of impurity in sample $(\mu g)$ : & 0.70 & $<0.66$ & $<1.36$ \\
\hline & Weight of impurity in blank $(\mu g)$ : & $<0.85$ & $<0.65$ & +2 \\
\hline & Minimum corrected weight of impurity in sample $(\mu g)$ : & 0.00 & 0.00 & 0.00 \\
\hline & Maximum corrected weight of impurity in sample $(\mu g)$ : & 0.70 & 0.66 & 1.36 \\
\hline \multirow{5}{*}{ Co } & Measured concentration of impurity in sample $(\mu \mathrm{g} / \mathrm{ml})$ : & $<4.06 \mathrm{E}-03$ & $<4.06 \mathrm{E}-03$ & Co \\
\hline & Uncorrected weight of impurity in sample $(\mu \mathrm{g})$ : & $<0.60$ & $<0.56$ & $<1.16$ \\
\hline & Weight of impurity in blank $(\mu g)$ : & $<0.72$ & $<0.56$ & 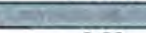 \\
\hline & Minimum corrected weight of impurity in sample $(\mu g)$ : & 0.00 & 0.00 & 0.00 \\
\hline & Maximum corrected weight of impurity in sample $(\mu g)$ : & 0.60 & 0.56 & 1.16 \\
\hline \multirow{5}{*}{$\mathrm{Ni}$} & Measured concentration of impurity in sample $(\mu \mathrm{g} / \mathrm{ml})$ : & $<2.00 E-02$ & $<2.00 E-02$ & $\mathrm{Ni}$ \\
\hline & Uncorrected weight of impurity in sample $(\mu g)$ : & $<2.94$ & $<2.76$ & $<5.70$ \\
\hline & Weight of impurity in blank $(\mu 9)$ : & $<3.56$ & $<2.74$ & 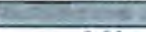 \\
\hline & Minimum corrected weight of impurity in sample $(\mu \mathrm{g})$ : & 0.00 & 0.00 & 0.00 \\
\hline & Maximum corrected weight of impurity in sample $(\mu g)$ : & 2.94 & 2.76 & 5.70 \\
\hline \multirow{5}{*}{$\mathrm{Ca}$} & Measured concentration of impurity in sample $(\mu \mathrm{g} / \mathrm{ml})$ : & $5.06 \mathrm{E}-01$ & $1.69 \mathrm{E}-01$ & $\mathrm{Ca}$ \\
\hline & Uncorrected weight of impurity in sample $(\mu g)$ : & 74.38 & 23.32 & 97.70 \\
\hline & Weight of impurity in blank $(\mu \mathrm{g})$ : & $<17.80$ & 42.61 & 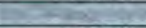 \\
\hline & Minimum corrected weight of impurity in sample $(\mu g)$ : & 56.58 & 0.00 & 56.58 \\
\hline & Maximum corrected weight of impurity in sample $(\mu g)$ : & 74.38 & 0.00 & 74.38 \\
\hline \multirow{5}{*}{ Al } & Measured concentration of impurity in sample $(\mu \mathrm{g} / \mathrm{ml})$ : & $4.47 E-01$ & $1.15 E-01$ & Al \\
\hline & Uncorrected weight of impurity in sample $(\mu g)$ : & 65.71 & 15.87 & 81.58 \\
\hline & Weight of impurity in blank $(\mu \mathrm{g})$ : & 5.46 & 3.45 & \\
\hline & Minimum corrected weight of impurity in sample $(\mu \mathrm{g})$ : & 60.24 & 12.42 & 72.66 \\
\hline & Maximum corrected weight of impurity in sample $(\mu g)$ : & 60.24 & 12.42 & 72.66 \\
\hline \multirow{5}{*}{$\mathbf{T i}$} & Measured concentration of impurity in sample $(\mu \mathrm{g} / \mathrm{ml})$ : & $3.72 \mathrm{E}-02$ & $<2.00 \mathrm{E}-02$ & Ti \\
\hline & Uncorrected weight of impurity in sample $(\mu \mathrm{g})$ : & 5.47 & $<2.76$ & $<8.23$ \\
\hline & Weight of impurity in blank $(\mu g)$ : & $<3.56$ & $<2.74$ & 8 \\
\hline & Minimum corrected weight of impurity in sample $(\mu g)$ : & 1.91 & 0.00 & 1.91 \\
\hline & Maximum corrected weight of impurity in sample $(\mu g)$ : & 5.47 & 2.76 & 8.23 \\
\hline \multirow{5}{*}{$\mathbf{v}$} & Measured concentration of impurity in sample $(\mu \mathrm{g} / \mathrm{ml})$ : & $2.40 \mathrm{E}-01$ & 5.37E-02 & $\mathbf{v}$ \\
\hline & Uncorrected weight of impurity in sample $(\mu \mathrm{g})$ : & 35.28 & 7.41 & 42.69 \\
\hline & Weight of impurity in blank $(\mu \mathrm{g})$ : & $<0.89$ & $<0.69$ & 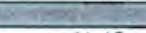 \\
\hline & Minimum corrected weight of impurity in sample $(\mu g)$ : & 34.39 & 6.73 & 41.12 \\
\hline & Maximum corrected weight of impurity in sample $(\mu g)$ : & 35.28 & 7.41 & 42.69 \\
\hline \multicolumn{5}{|c|}{ Comments } \\
\hline \multicolumn{5}{|c|}{ FCM Checked the official results of analyses for RMAL1881 on $7 / 10 / 2009$} \\
\hline
\end{tabular}

\section{Foed c. Montromery}

$7-23-09$ 
Data Report Form DRF-26A: Measurement of U Contamination or Impurities by Deconsolidation Leach

\begin{tabular}{|c|c|}
\hline Procedure: & AGR-CHAR-DAM-26 Rev. 1 \\
\hline Operator: & Fred Montgomery \\
\hline Compact lot ID: & LEU07-OP1-Z \\
\hline Compact lot description: & AGR-2 UCO Baseline Fuel, from G73J-14-93072A \\
\hline Compact ID numbers: & Deconsolidation Leach Blank \\
\hline DRF filename: & IImc-agr\AGR\LeachBurnLeach\LEU07-OP1-Z_DRF26R1_01.xIs \\
\hline
\end{tabular}

\begin{tabular}{|r|c|}
\hline Mean average weight uranium per particle $(\mathrm{g}):$ & $3.93 \mathrm{E}-04$ \\
\hline Uncertainty in mean average weight uranium per particle $(\mathrm{g}):$ & $6.56 \mathrm{E}-07$ \\
\hline
\end{tabular}

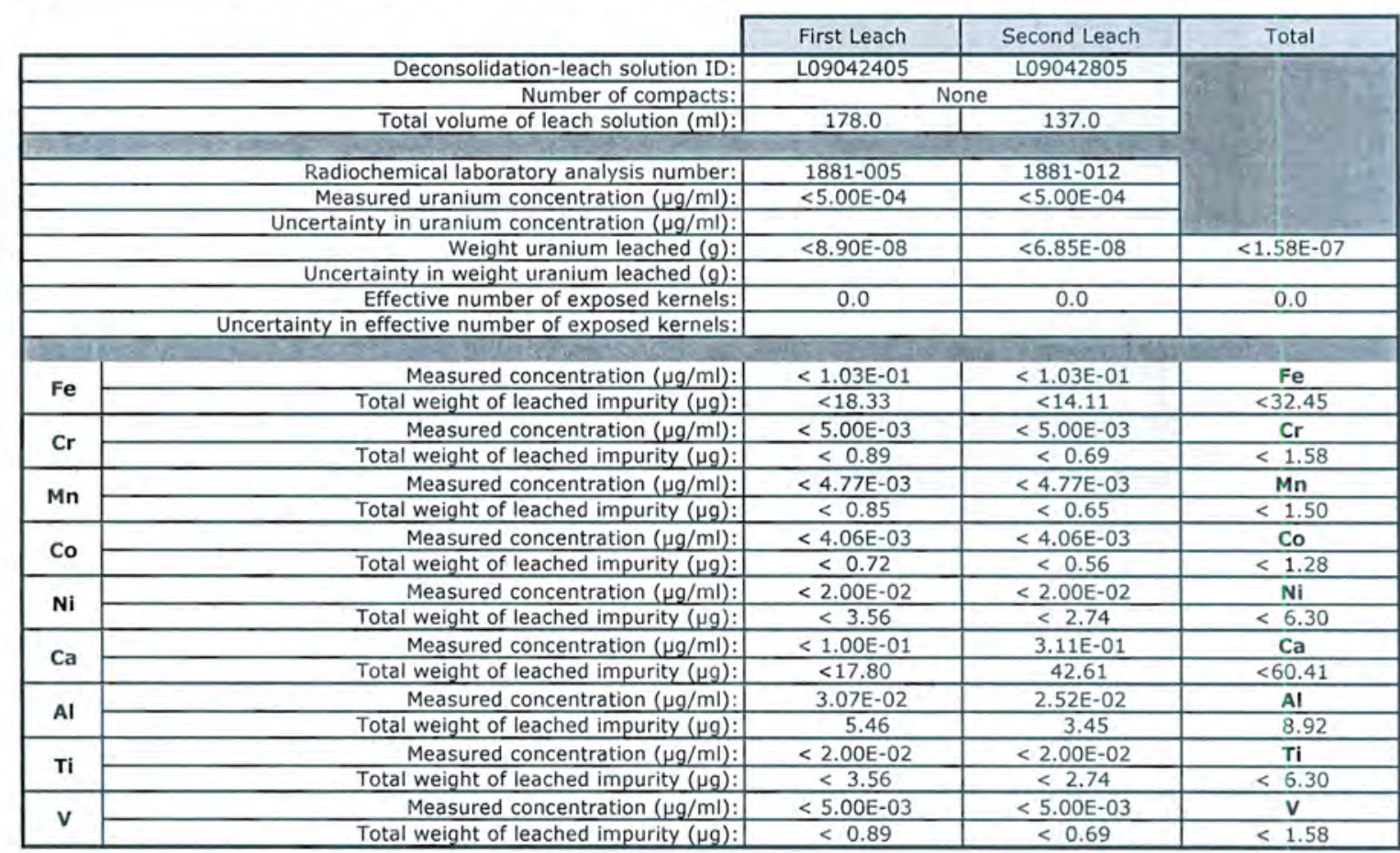

Comments

FCM Checked the official results of analyses for RMAL1881 on 7/10/2009 
Data Report Form DRF-26B: Measurement of SIC Burn-Leach Defects or Impurities by Burn-Leach

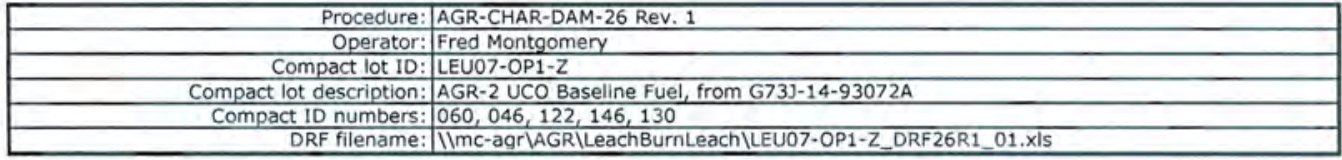

\begin{tabular}{|c|c|c|c|c|}
\hline \multicolumn{2}{|r|}{$\begin{array}{r}\text { Mean average weight uranium per particle }(q): \\
\text { Uncertainty in mean average weight uranium per particle }(q):\end{array}$} & \multicolumn{3}{|c|}{$\frac{3.93 E-04}{6.56 E-07}$} \\
\hline & & First Leach & Second Leach & Total \\
\hline \multirow{2}{*}{\multicolumn{2}{|c|}{$\begin{array}{l}\text { Burn-leach solution ID: } \\
\text { Number of compacts: }\end{array}$}} & 809051101 & B09051206 & \\
\hline & & & & \\
\hline \multicolumn{2}{|r|}{ Total volume of leach solution $(\mathrm{ml})$ : } & 52.0 & 55.5 & \\
\hline \multicolumn{2}{|r|}{ Radiochemical laboratory analysis number: } & $1910-011$ & $1918-006$ & \\
\hline \multirow{2}{*}{\multicolumn{2}{|c|}{$\begin{array}{l}\text { Measured uranium concentration }(\mu \mathrm{g} / \mathrm{ml}): \\
\text { Uncertainty in uranium concentration }(\mu \mathrm{g} / \mathrm{ml}):\end{array}$}} & $4.48 \mathrm{E}-01$ & $6.64 \mathrm{E}-03$ & \\
\hline & & $4.48 \mathrm{E}-02$ & $6.64 \mathrm{E}-04$ & \\
\hline \multicolumn{2}{|r|}{ Weight uranium leached $(g)$ : } & $2.33 \mathrm{E}-05$ & $3.69 \mathrm{E}-07$ & $2.37 E-05$ \\
\hline \multicolumn{2}{|r|}{ Uncertainty in weight uranium leached $(g)$ : } & $2.35 E-06$ & $3.71 \mathrm{E}-08$ & $2.35 \mathrm{E}-06$ \\
\hline \multirow{2}{*}{\multicolumn{2}{|c|}{$\begin{array}{l}\text { Number of leached kernels: } \\
\text { Uncertainty in number of leached kernels: }\end{array}$}} & 0.1 & 0.0 & 0.1 \\
\hline & & 0.0 & 0.0 & 0.0 \\
\hline & & & & \\
\hline \multirow{4}{*}{$\mathrm{Fe}$} & $\frac{\text { Measured concentration of impurity in sample }(\mu \mathrm{g} / \mathrm{ml}) \text { : }}{\text { Uncorrected weight of impurity in sample }(\mu \mathrm{g}):}$ & $\frac{1.12 \mathrm{E}-01}{5.82}$ & $\frac{<4.12 \mathrm{E}-02}{<2.29}$ & $\frac{\mathrm{Fe}}{<8.11}$ \\
\hline & Weight of impurity in blank $(\mu g)$ : & 3.46 & $<2.22$ & \\
\hline & Minimum corrected weight of impurity in sample $(\mu \mathrm{g})$ : & 2.37 & 0.00 & 2.37 \\
\hline & Maximum corrected weight of impurity in sample $(\mu g)$ : & 2.37 & 2.29 & 4.65 \\
\hline \multirow{5}{*}{$\mathrm{Cr}$} & Measured concentration of impurity in sample $(\mu \mathrm{g} / \mathrm{ml})$ : & $1.57 E-02$ & $<2.00 \mathrm{E}-03$ & $\mathrm{Cr}$ \\
\hline & Uncorrected weight of impurity in sample $(\mu g)$ : & 0.82 & $<0.11$ & $<0.93$ \\
\hline & Weight of impurity in blank $(\mu \mathrm{g})$ : & 0.20 & $<0.11$ & \\
\hline & Minimum corrected weight of impurity in sample $(\mu g)$ : & 0.62 & 0.00 & 0.62 \\
\hline & Maximum corrected weight of impurity in sample $(\mu g)$ : & 0.62 & 0.11 & 0.73 \\
\hline \multirow{5}{*}{ Mn } & Measured concentration of impurity in sample $(\mathrm{\mu g} / \mathrm{ml})$ : & $<1,91 \mathrm{E}-03$ & $<1.91 \mathrm{E}-03$ & $\mathrm{Mn}$ \\
\hline & Uncorrected weight of impurity in sample $(\mu \mathrm{g})$ : & $<0.10$ & $<0.11$ & $<0.21$ \\
\hline & Weight of impurity in blank $(\mu \mathrm{g})$ : & $<0.10$ & $<0.10$ & \\
\hline & Minimum corrected weight of impurity in sample $(\mu q)$ : & 0.00 & 0.00 & 0.00 \\
\hline & Maximum corrected weight of impurity in sample $(\mu g)$ : & 0.10 & 0.11 & 0.21 \\
\hline \multirow{5}{*}{ co } & Measured concentration of impurity in sample $(\mathrm{\mu g} / \mathrm{ml})$ : & $<1.62 \mathrm{E}-03$ & $<1.62 \mathrm{E}-03$ & Co \\
\hline & Uncorrected weight of impurity in sample $(\mu g)$ : & $<0.08$ & $<0.09$ & $<0.17$ \\
\hline & Weight of impurity in blank $(\mu g)$ : & 0.29 & $<0.09$ & \\
\hline & Minimum corrected weight of impurity in sample $(\mu g)$ : & 0.00 & 0.00 & 0.00 \\
\hline & Maximum corrected weight of impurity in sample $(\mu g)$ : & 0.00 & 0.09 & 0.09 \\
\hline \multirow{5}{*}{$\mathrm{Ni}$} & Measured concentration of impurity in sample $(\mu \mathrm{g} / \mathrm{ml})$ : & $9.92 \mathrm{E}-03$ & $<8.00 \mathrm{E}-03$ & \\
\hline & Uncorrected weight of impurity in sample $(\mu g)$ : & 0.52 & $<0.44$ & $<0.96$ \\
\hline & Weight of impurity in blank $(\mu \mathrm{g})$ : & $<0.41$ & $<0.43$ & \\
\hline & Minimum corrected weight of impurity in sample $(\mu \mathrm{g})$ : & 0.10 & 0.00 & 0.10 \\
\hline & Maximum corrected weight of impurity in sample $(u g)$ : & 0.52 & 0.44 & 0.96 \\
\hline \multirow{5}{*}{$\mathrm{Ca}$} & Measured concentration of impurity in sample $(\mu \mathrm{g} / \mathrm{ml})$ : & $2.16 \mathrm{E}+00$ & $1.15 \mathrm{E}-01$ & $\mathrm{Ca}$ \\
\hline & Uncorrected weight of impurity in sample $(\mu \mathrm{g})$ : & 112.32 & 6.38 & 118.70 \\
\hline & Weight of impurity in blank $(\mu \mathrm{g})$ : & $<5.15$ & $<5.40$ & \\
\hline & Minimum corrected weight of impurity in sample $(\mu g)$ : & 107.17 & 0.98 & 108.15 \\
\hline & Maximum corrected weight of impurity in sample $(\mu g)$ : & 112.32 & 6.38 & 118.70 \\
\hline \multirow{5}{*}{ Al } & Measured concentration of impurity in sample $(\mathrm{\mu g} / \mathrm{ml})$ : & $1.42 E+00$ & $4.64 \mathrm{E}-02$ & Al \\
\hline & Uncorrected weight of impurity in sample $(\mu g)$ : & 73.84 & 2.58 & 76.42 \\
\hline & Weight of impurity in blank $(\mu \mathrm{g})$ : & 2.60 & 0.71 & \\
\hline & Minimum corrected weight of impurity in sample $(\mu g)$ : & 71.24 & 1.86 & 73.10 \\
\hline & Maximum corrected weight of impurity in sample $(\mu g)$ : & 71.24 & 1.86 & 73.10 \\
\hline \multirow{5}{*}{$\mathrm{Ti}$} & Measured concentration of impurity in sample $(\mu \mathrm{g} / \mathrm{ml})$ : & $1.62 \mathrm{E}-01$ & $<8.00 \mathrm{E}-03$ & $\mathrm{Ti}$ \\
\hline & Uncorrected weight of impurity in sample $(\mu \mathrm{g})$ : & 8.42 & $<0.44$ & $<8.87$ \\
\hline & Weight of impurity in blank $(\mu g)$ : & $<0.41$ & $<0.43$ & \\
\hline & Minimum corrected weight of impurity in sample $(\mu g)$ : & 8.01 & 0.00 & 8.01 \\
\hline & Maximum corrected weight of impurity in sample $(\mu g)$ : & 8.42 & 0.44 & 8.87 \\
\hline \multirow{5}{*}{ v } & Measured concentration of impurity in sample $(\mu \mathrm{g} / \mathrm{ml})$ : & $1.03 E+00$ & $6.74 \mathrm{E}-03$ & $\mathrm{v}$ \\
\hline & Uncorrected weight of impurity in sample $(\mu \mathrm{g})$ : & 53.56 & 0.37 & 53.93 \\
\hline & Weight of impurity in blank $(\mu g)$ : & $<0.10$ & $<0.11$ & \\
\hline & Minimum corrected weight of impurity in sample $(\mu g)$ : & 53.46 & 0.27 & 53.72 \\
\hline & Maximum corrected weight of impurity in sample $(\mu q)$ : & 53.56 & 0.37 & 53.93 \\
\hline
\end{tabular}

Comments

Feed c montfanney
$7-23-09$ 
Data Report Form DRF-26B: Measurement of SiC Burn-Leach Defects or Impurities by Burn-Leach

\begin{tabular}{|c|c|}
\hline Procedure: & AGR-CHAR-DAM-26 Rev. 1 \\
\hline Operator: & Fred Montgomery \\
\hline $\begin{array}{r}\text { Compact lot ID: } \\
\text { Compact lot description: }\end{array}$ & $\begin{array}{l}\text { LEU07-OP1-Z } \\
\text { AGR-2 UCO Baseline Fuel from G73]-14-93072A }\end{array}$ \\
\hline Compact ID numbers: & $141,138,111,064,053$ \\
\hline DRF filename: & IImc-agr|AGRILeachBurnLeach|LEU07-OP1-Z_DRF26R1_01.x|s \\
\hline
\end{tabular}

\begin{tabular}{|c|c|c|c|c|}
\hline & $\begin{array}{l}\text { Mean average weight uranium per particle }(g) \text { : } \\
\text { Uncertainty in mean average weight uranium per particle }(q) \text { : }\end{array}$ & & $\begin{array}{l}3.93 \mathrm{E}-04 \\
6.56 \mathrm{E}-07\end{array}$ & \\
\hline & & First Leach & Second Leach & Total \\
\hline & Burn-leach solution ID: & 809051102 & B09051207 & \\
\hline & Number of compacts: & & & \\
\hline & Total volume of leach solution $(\mathrm{ml})$ : & 53.0 & 54.0 & \\
\hline & +2 & & & \\
\hline & Radiochemical laboratory analysis number: & $1910-012$ & $1918-007$ & \\
\hline & Measured uranium concentration $(\mathrm{\mu g} / \mathrm{ml})$ : & $4.43 \mathrm{E}-01$ & $7.12 \mathrm{E}-03$ & \\
\hline & Uncertainty in uranium concentration $(\mu \mathrm{g} / \mathrm{ml})$ : & $4.43 \mathrm{E}-02$ & $7.12 \mathrm{E}-04$ & \\
\hline & Weight uranium leached (g): & $2.35 E-05$ & $3.84 E-07$ & $2.39 \mathrm{E}-05$ \\
\hline & Uncertainty in weight uranium leached $(g)$ : & $2.37 E-06$ & $3.87 E-08$ & $2.37 E-06$ \\
\hline & Number of leached kernels: & 0.1 & 0.0 & 0.1 \\
\hline & Uncertainty in number of leached kernels: & 0.0 & 0.0 & 0.0 \\
\hline & Measured concentration of impurity in sample $(\mu \mathrm{g} / \mathrm{ml})$ : & 4.06E-01 & $<4.12 \mathrm{E}-02$ & $\mathrm{Fe}$ \\
\hline & Uncorrected weight of impurity in sample $(\mu g)$ : & 21.52 & $<2.22$ & $<23.74$ \\
\hline $\mathrm{Fe}$ & Weight of impurity in blank $(\mu \mathrm{g})$ : & 3.46 & $<2.22$ & \\
\hline & Minimum corrected weight of impurity in sample $(\mu q)$ : & 18.06 & 0.00 & 18.06 \\
\hline & Maximum corrected weight of impurity in sample $(\mu g)$ : & 18.06 & 2.22 & 20.29 \\
\hline & Measured concentration of impurity in sample $(\mu \mathrm{g} / \mathrm{ml})$ : & $1.52 \mathrm{E}-02$ & $<2.00 \mathrm{E}-03$ & $\mathrm{Cr}$ \\
\hline & Uncorrected weight of impurity in sample $(\mu g)$ : & 0.81 & $<0.11$ & $<0.91$ \\
\hline $\mathrm{Cr}$ & Weight of impurity in blank $(\mu \mathrm{g})$ : & 0.20 & $<0.11$ & \\
\hline & Minimum corrected weight of impurity in sample $(\mu g)$ : & 0.61 & 0.00 & 0.61 \\
\hline & Maximum corrected weight of impurity in sample $(\mu \mathrm{g})$ : & 0.61 & 0.11 & 0.72 \\
\hline & Measured concentration of impurity in sample $(\mathrm{\mu g} / \mathrm{ml})$ : & $<1.91 \mathrm{E}-03$ & $<1.91 \mathrm{E}-03$ & $\mathrm{Mn}$ \\
\hline & Uncorrected weight of impurity in sample $(\mu \mathrm{g})$ : & $<0.10$ & $<0.10$ & $<0.20$ \\
\hline Mn & Weight of impurity in blank $(\mu \mathrm{g})$ : & $<0.10$ & $<0.10$ & \\
\hline & Minimum corrected weight of impurity in sample $(\mu g)$ : & 0.00 & 0.00 & 0.00 \\
\hline & Maximum corrected weight of impurity in sample $(\mu g)$ : & 0.10 & 0.10 & 0.20 \\
\hline & Measured concentration of impurity in sample $(\mu \mathrm{g} / \mathrm{ml})$ : & $1.62 \mathrm{E}-03$ & $<1.62 \mathrm{E}-03$ & Co \\
\hline & Uncorrected weight of impurity in sample $(\mu g)$ : & 0.09 & $<0.09$ & $<0.17$ \\
\hline Co & Weight of impurity in blank $(\mu \mathrm{g})$ : & 0.29 & $<0.09$ & \\
\hline & Minimum corrected weight of impurity in sample $(\mu g)$ : & 0.00 & 0.00 & 0.00 \\
\hline & Maximum corrected weight of impurity in sample $(\mu \mathrm{g})$ : & 0.00 & 0.09 & 0.09 \\
\hline & Measured concentration of impurity in sample $(\mathrm{\mu g} / \mathrm{ml})$ : & $9.64 \mathrm{E}-03$ & $<8.00 \mathrm{E}-03$ & $\mathrm{Ni}$ \\
\hline & Uncorrected weight of impurity in sample $(\mu \mathrm{g})$ : & 0.51 & $<0.43$ & $<0.94$ \\
\hline $\mathrm{Ni}$ & Weight of impurity in blank $(\mu \mathrm{g})$ : & $<0.41$ & $<0.43$ & \\
\hline & Minimum corrected weight of impurity in sample $(\mu g)$ : & 0.10 & 0.00 & 0.10 \\
\hline & Maximum corrected weight of impurity in sample $(\mu g)$ : & 0.51 & 0.43 & 0.94 \\
\hline & Measured concentration of impurity in sample $(\mu \mathrm{g} / \mathrm{ml})$ : & $1.98 \mathrm{E}+00$ & $2.98 \mathrm{E}-01$ & $\mathrm{Ca}$ \\
\hline & Uncorrected weight of impurity in sample $(\mu g)$ : & 104.94 & 16.09 & 121.03 \\
\hline $\mathrm{Ca}$ & Weight of impurity in blank $(\mu \mathrm{g})$ : & $<5.15$ & $<5.40$ & \\
\hline & Minimum corrected weight of impurity in sample $(\mu g)$ : & 99.79 & 10.69 & 110.48 \\
\hline & Maximum corrected weight of impurity in sample $(\mu \mathrm{g})$ : & 104.94 & 16.09 & 121.03 \\
\hline & Measured concentration of impurity in sample $(\mathrm{\mu g} / \mathrm{ml})$ : & $1.36 \mathrm{E}+00$ & $5.16 \mathrm{E}-02$ & Al \\
\hline & Uncorrected weight of impurity in sample $(\mu g)$ : & 72.08 & 2.79 & 74.87 \\
\hline Al & Weight of impurity in blank $(\mu g)$ : & 2.60 & 0.71 & \\
\hline & Minimum corrected weight of impurity in sample $(\mu \mathrm{g})$ : & 69.48 & 2.07 & 71.55 \\
\hline & Maximum corrected weight of impurity in sample $(\mu \mathrm{g})$ : & 69.48 & 2.07 & 71.55 \\
\hline & Measured concentration of impurity in sample $(\mu \mathrm{g} / \mathrm{ml})$ : & $1.33 \mathrm{E}-01$ & $<8.00 \mathrm{E}-03$ & $\mathrm{Ti}$ \\
\hline & Uncorrected weight of impurity in sample $(\mu g)$ : & 7.05 & $<0.43$ & $<7.48$ \\
\hline $\mathbf{T i}$ & Weight of impurity in blank $(\mathrm{\mu g})$ : & $<0.41$ & $<0.43$ & \\
\hline & Minimum corrected weight of impurity in sample $(\mu g)$ : & 6.64 & 0.00 & 6.64 \\
\hline & Maximum corrected weight of impurity in sample $(\mu g)$ : & 7.05 & 0.43 & 7.48 \\
\hline & Measured concentration of impurity in sample $(\mu \mathrm{g} / \mathrm{ml})$ : & $1.00 E+00$ & $7.22 \mathrm{E}-03$ & $\mathrm{v}$ \\
\hline & Uncorrected weight of impurity in sample $(\mu \mathrm{g})$ : & 53.00 & 0.39 & 53.39 \\
\hline v & Weight of impurity in blank $(\mathrm{\mu g})$ : & $<0.10$ & $<0.11$ & \\
\hline & Minimum corrected weight of impurity in sample $(\mu \mathrm{g})$ : & 52.90 & 0.28 & 53.18 \\
\hline & Maximum corrected weight of impurity in sample $(\mu g)$ : & 53.00 & 0.39 & 53.39 \\
\hline
\end{tabular}

Comments

FCM Checked the official results of analyses for RMAL1910 and RMAL1918 on 7/10/2009

Foed C. mentgomery $7-23-09$ 
Data Report Form DRF-26B: Measurement of SIC Burn-Leach Defects or Impurities by Burn-Leach

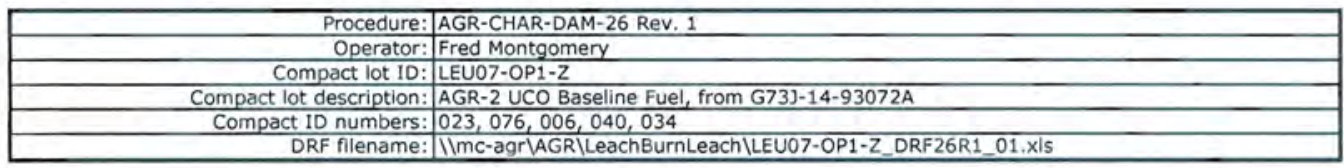

\begin{tabular}{|c|c|c|c|c|}
\hline & $\begin{array}{l}\text { Mean average weight uranium per particle }(g) \text { : } \\
\text { Uncertainty in mean average weight uranium per particle }(q) \text { : }\end{array}$ & & $\frac{3.93 E-04}{6.56 E-07}$ & \\
\hline & & First Leach & Second Leach & Total \\
\hline & Burn-leach solution ID: & B09051103 & $B 09051208$ & \\
\hline & Number of compacts: & & & \\
\hline & Total volume of leach solution $(\mathrm{ml})$ : & 52.5 & 57.0 & \\
\hline & Radiochemical laboratory analysis number: & & & \\
\hline & $\begin{array}{l}\text { Radiochemical laboratory analysis number: } \\
\text { Measured uranium concentration }(\mu \mathrm{g} / \mathrm{ml}):\end{array}$ & $\frac{1910-013}{4.39 E-01}$ & $\frac{1918-008}{5.86 \mathrm{E}-03}$ & \\
\hline & Uncertainty in uranium concentration $(\mathrm{\mu g} / \mathrm{ml})$ : & $4.39 \mathrm{E}-02$ & $5.86 \mathrm{E}-04$ & \\
\hline & Weight uranium leached $(\mathrm{g})$ : & $2.30 \mathrm{E}-05$ & $3.34 \mathrm{E}-07$ & $2.34 \mathrm{E}-05$ \\
\hline & Uncertainty in weight uranium leached $(g)$ : & $2.32 \mathrm{E}-06$ & $3.36 \mathrm{E}-08$ & $2.32 \mathrm{E}-06$ \\
\hline & Number of leached kernels: & 0.1 & 0.0 & 0.1 \\
\hline & Uncertainty in number of leached kernels: & 0.0 & 0.0 & 0.0 \\
\hline & Measured concentration of impurity in sample $(\mathrm{\mu g} / \mathrm{ml})$ : & $8.01 \mathrm{E}-02$ & $<4.12 \mathrm{E}-02$ & $\mathrm{Fe}$ \\
\hline & Uncorrected weight of impurity in sample $(\mu q)$ : & 4.21 & $<2.35$ & $<6.55$ \\
\hline $\mathrm{Fe}$ & Weight of impurity in blank $(\mu g)$ : & 3.46 & $<2.22$ & \\
\hline & Minimum corrected weight of impurity in sample $(\mu g)$ : & 0.75 & 0.00 & 0.75 \\
\hline & Maximum corrected weight of impurity in sample $(\mu g)$ : & 0.75 & 2.35 & 3.10 \\
\hline & Measured concentration of impurity in sample $(\mathrm{\mu g} / \mathrm{ml})$ : & $1.50 \mathrm{E}-02$ & $<2.00 \mathrm{E}-03$ & $\mathrm{Cr}$ \\
\hline & Uncorrected weight of impurity in sample $(\mu g)$ : & 0.79 & $<0.11$ & $<0.90$ \\
\hline $\mathrm{Cr}$ & Weight of impurity in blank $(\mu \mathrm{g})$ : & 0.20 & $<0.11$ & \\
\hline & Minimum corrected weight of impurity in sample $(\mu g)$ : & 0.59 & 0.00 & 0.59 \\
\hline & Maximum corrected weight of impurity in sample $(\mu g)$ : & 0.59 & 0.11 & 0.70 \\
\hline & Measured concentration of impurity in sample $(\mathrm{\mu g} / \mathrm{ml})$ : & $<1.91 \mathrm{E}-03$ & $<1,91 \mathrm{E}-03$ & $\mathrm{Mn}$ \\
\hline & Uncorrected weight of impurity in sample $(\mu \mathrm{g})$ : & $<0.10$ & $<0.11$ & $<0.21$ \\
\hline $\mathrm{Mn}$ & Weight of impurity in blank $(\mu \mathrm{g})$ : & $<0.10$ & $<0.10$ & \\
\hline & Minimum corrected weight of impurity in sample $(\mu g)$ : & 0.00 & 0.00 & 0.00 \\
\hline & Maximum corrected weight of impurity in sample $(\mu g)$ : & 0.10 & 0.11 & 0.21 \\
\hline & Measured concentration of impurity in sample $(\mathrm{\mu g} / \mathrm{ml})$ : & $<1.62 \mathrm{E}-03$ & $<1.62 \mathrm{E}-03$ & Co \\
\hline & Uncorrected weight of impurity in sample $(\mu g)$ : & $<0.09$ & $<0.09$ & $<0.18$ \\
\hline Co & Weight of impurity in blank $(\mu \mathrm{g})$ : & 0.29 & $<0.09$ & $=0.10$ \\
\hline & Minimum corrected weight of impurity in sample $(\mu g)$ : & 0.00 & 0.00 & 0.00 \\
\hline & Maximum corrected weight of impurity in sample $(\mu g)$ : & 0.00 & 0.09 & 0.09 \\
\hline & Measured concentration of impurity in sample $(\mathrm{\mu g} / \mathrm{ml})$ : & $8.96 \mathrm{E}-03$ & $<8.00 \mathrm{E}-03$ & $\mathrm{Ni}$ \\
\hline & Uncorrected weight of impurity in sample $(\mu \mathrm{g})$ : & 0.47 & $<0.46$ & $<0.93$ \\
\hline $\mathrm{Ni}$ & Weight of impurity in blank $(\mu \mathrm{g})$ : & $<0.41$ & $<0.43$ & \\
\hline & Minimum corrected weight of impurity in sample $(\mu g)$ : & 0.06 & 0.00 & 0.06 \\
\hline & Maximum corrected weight of impurity in sample $(u g)$ : & 0.47 & 0.46 & 0.93 \\
\hline & Measured concentration of impurity in sample $(\mu \mathrm{g} / \mathrm{ml})$ : & $1.98 E+00$ & $1.49 \mathrm{E}-01$ & $\mathrm{Ca}$ \\
\hline & Uncorrected weight of impurity in sample $(\mu \mathrm{g})$ : & 103.95 & 8.49 & 112.44 \\
\hline Ca & Weight of impurity in blank $(\mu \mathrm{g})$ : & $<5.15$ & $<5.40$ & \\
\hline & Minimum corrected weight of impurity in sample $(\mu g)$ : & 98.80 & 3.09 & 101.89 \\
\hline & Maximum corrected weight of impurity in sample $(\mu \mathrm{g})$ : & 103.95 & 8.49 & 112.44 \\
\hline & Measured concentration of impurity in sample $(\mathrm{\mu g} / \mathrm{ml})$ : & $1.42 \mathrm{E}+00$ & $4.51 \mathrm{E}-02$ & Al \\
\hline & Uncorrected weight of impurity in sample $(\mu \mathrm{g})$ : & 74.55 & 2.57 & 77.12 \\
\hline Al & Weight of impurity in blank $(\mu g)$ : & 2.60 & 0.71 & \\
\hline & Minimum corrected weight of impurity in sample $(\mu g)$ : & 71.95 & 1.86 & 73.81 \\
\hline & Maximum corrected weight of impurity in sample $(\mu g)$ : & 71.95 & 1.86 & 73.81 \\
\hline & Measured concentration of impurity in sample $(\mu \mathrm{g} / \mathrm{ml})$ : & $1.83 E-01$ & $<8.00 \mathrm{E}-03$ & $\mathrm{Ti}$ \\
\hline & Uncorrected weight of impurity in sample $(\mu \mathrm{g})$ : & 9.61 & $<0.46$ & $<10.06$ \\
\hline $\mathrm{Ti}$ & Weight of impurity in blank $(\mu g)$ : & $<0.41$ & $<0.43$ & \\
\hline & Minimum corrected weight of impurity in sample $(\mu g)$ : & 9.20 & 0.00 & 9.20 \\
\hline & Maximum corrected weight of impurity in sample $(\mu \mathrm{g})$ : & 9.61 & 0.46 & 10.06 \\
\hline & Measured concentration of impurity in sample $(\mu \mathrm{g} / \mathrm{ml})$ : & $1.04 E+00$ & $5.59 \mathrm{E}-03$ & $\mathrm{v}$ \\
\hline & Uncorrected weight of impurity in sample $(\mu g)$ : & 54.60 & 0.32 & 54.92 \\
\hline $\mathbf{v}$ & Weight of impurity in blank $(\mu \mathrm{g})$ : & $<0.10$ & $<0.11$ & \\
\hline & Minimum corrected weight of impurity in sample $(\mu g)$ : & 54.50 & 0.21 & 54.71 \\
\hline & Maximum corrected weight of impurity in sample $(\mu g)$ : & 54.60 & 0.32 & 54.92 \\
\hline
\end{tabular}

Comments

FCM Checked the official results of analyses for RMAL1910 and RMAL1918 on 7/10/2009

\section{Fied C. Monteomey 7-23-09}


Data Report Form DRF-26B: Measurement of SIC Burn-Leach Defects or Impurities by Burn-Leach

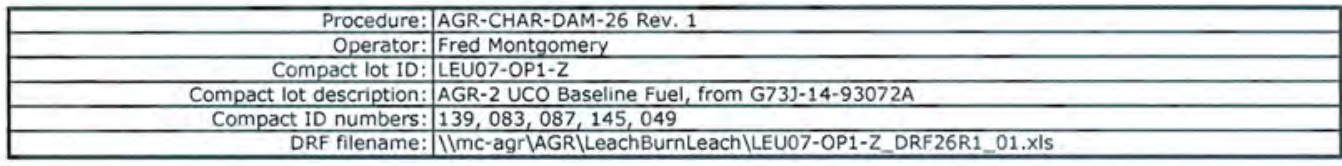

\begin{tabular}{|c|c|c|c|c|}
\hline & $\begin{array}{l}\text { Mean average weight uranium per particle }(\mathrm{g}) \text { : } \\
\text { Uncertainty in mean average weight uranium per particle }(q) \text { : }\end{array}$ & & $\frac{3.93 E-04}{6.56 E-07}$ & \\
\hline & & First Leach & Second Leach & Total \\
\hline & Burn-leach solution ID: & $B 09051104$ & 809051209 & \\
\hline & Number of compacts: & & & \\
\hline & Total volume of leach solution (ml): & 49.0 & 52.5 & \\
\hline & Radiochemical laboratory analysis number: & $1910-014$ & $1918-009$ & \\
\hline & Measured uranium concentration $(\mathrm{\mu g} / \mathrm{ml})$ : & $4.99 \mathrm{E}-01$ & $6.31 \mathrm{E}-03$ & \\
\hline & Uncertainty in uranium concentration $(\mathrm{\mu g} / \mathrm{ml})$ : & $4.99 \mathrm{E}-02$ & $6.31 \mathrm{E}-04$ & \\
\hline & Weight uranium leached $(g)$ : & $2.45 E-05$ & $3.31 \mathrm{E}-07$ & $2.48 \mathrm{E}-05$ \\
\hline & Uncertainty in weight uranium leached $(\mathrm{g})$ : & $2.47 \mathrm{E}-06$ & $3.34 E-08$ & $2.47 \mathrm{E}-06$ \\
\hline & Number of leached kernels: & 0.1 & 0.0 & 0.1 \\
\hline & Uncertainty in number of leached kernels: & 0.0 & 0.0 & 0.0 \\
\hline & Measured concentration of impurity in sample $(\mu \mathrm{g} / \mathrm{ml})$ : & $8.73 E-02$ & $<4.12 \mathrm{E}-02$ & \\
\hline & Uncorrected weight of impurity in sample $(\mu \mathrm{g})$ : & 4.28 & $<2.16$ & $<6.44$ \\
\hline $\mathbf{F e}$ & Weight of impurity in blank $(\mu \mathrm{g})$ : & 3.46 & $<2.22$ & \\
\hline & Minimum corrected weight of impurity in sample $(\mu \mathrm{g})$ : & 0.82 & 0.00 & 0.82 \\
\hline & Maximum corrected weight of impurity in sample $(\mathrm{\mu g})$ : & 0.82 & 2.16 & 2.99 \\
\hline & Measured concentration of impurity in sample $(\mu \mathrm{g} / \mathrm{ml})$ : & $1.49 \mathrm{E}-02$ & $<2.00 E-03$ & $\mathrm{Cr}$ \\
\hline & Uncorrected weight of impurity in sample $(\mu \mathrm{g})$ : & 0.73 & $<0.11$ & $<0.84$ \\
\hline $\mathrm{Cr}$ & Weight of impurity in blank $(\mu \mathrm{g})$ : & 0.20 & $<0.11$ & \\
\hline & Minimum corrected weight of impurity in sample $(\mu \mathrm{g})$ : & 0.53 & 0.00 & 0.53 \\
\hline & Maximum corrected weight of impurity in sample $(\mu g)$ : & 0.53 & 0.11 & 0.64 \\
\hline & Measured concentration of impurity in sample $(\mu \mathrm{g} / \mathrm{ml})$ : & $<1.91 \mathrm{E}-03$ & $<1.91 \mathrm{E}-03$ & $\mathrm{Mn}$ \\
\hline & Uncorrected weight of impurity in sample $(\mu \mathrm{g})$ : & $<0.09$ & $<0.10$ & $<0.19$ \\
\hline Mn & Weight of impurity in blank $(\mu \mathrm{g})$ : & $<0.10$ & $<0.10$ & \\
\hline & Minimum corrected weight of impurity in sample $(\mu \mathrm{g})$ : & 0.00 & 0.00 & 0.00 \\
\hline & Maximum corrected weight of impurity in sample $(\mu \mathrm{g})$ : & 0.09 & 0.10 & 0.19 \\
\hline & Measured concentration of impurity in sample $(\mathrm{\mu g} / \mathrm{ml})$ : & $<1.62 \mathrm{E}-03$ & $<1.62 \mathrm{E}-03$ & Co \\
\hline & Uncorrected weight of impurity in sample $(\mu \mathrm{g})$ : & $<0.08$ & $<0.09$ & $<0.16$ \\
\hline Co & Weight of impurity in blank $(\mu g)$ : & 0.29 & $<0.09$ & \\
\hline & Minimum corrected weight of impurity in sample $(\mu g)$ : & 0.00 & 0.00 & 0.00 \\
\hline & Maximum corrected weight of impurity in sample $(\mu \mathrm{g})$ : & 0.00 & 0.09 & 0.09 \\
\hline & Measured concentration of impurity in sample $(\mu \mathrm{g} / \mathrm{ml})$ : & $8.30 \mathrm{E}-03$ & $<8.00 \mathrm{E}-03$ & $\mathrm{Ni}$ \\
\hline & Uncorrected weight of impurity in sample $(\mu g)$ : & 0.41 & $<0.42$ & $<0.83$ \\
\hline $\mathrm{Ni}$ & Weight of impurity in blank $(\mu \mathrm{g})$ : & $<0.41$ & $<0.43$ & \\
\hline & Minimum corrected weight of impurity in sample $(\mu \mathrm{g})$ : & 0.00 & 0.00 & 0.00 \\
\hline & Maximum corrected weight of impurity in sample $(\mu \mathrm{g})$ : & 0.41 & 0.42 & 0.83 \\
\hline & Measured concentration of impurity in sample $(\mu \mathrm{g} / \mathrm{ml})$ : & $1.95 E+00$ & $1.29 \mathrm{E}-01$ & $\mathrm{Ca}$ \\
\hline & Uncorrected weight of impurity in sample $(\mu \mathrm{g})$ : & 95.55 & 6.77 & 102.32 \\
\hline $\mathrm{Ca}$ & Weight of impurity in blank $(\mu g)$ : & $<5.15$ & $<5.40$ & \\
\hline & Minimum corrected weight of impurity in sample $(\mu g)$ : & 90.40 & 1.37 & 91.77 \\
\hline & Maximum corrected weight of impurity in sample $(\mu \mathrm{g})$ : & 95.55 & 6.77 & 102.32 \\
\hline & Measured concentration of impurity in sample $(\mu \mathrm{g} / \mathrm{ml})$ : & $1.48 \mathrm{E}+00$ & $3.74 \mathrm{E}-02$ & Al \\
\hline & Uncorrected weight of impurity in sample $(\mu \mathrm{g})$ : & 72.52 & 1.96 & 74.48 \\
\hline Al & Weight of impurity in blank $(\mu \mathrm{g})$ : & 2.60 & 0.71 & \\
\hline & Minimum corrected weight of impurity in sample $(\mu g)$ : & 69.92 & 1.25 & 71.17 \\
\hline & Maximum corrected weight of impurity in sample $(\mu \mathrm{g} g)$ : & 69.92 & 1.25 & 71.17 \\
\hline & Measured concentration of impurity in sample $(\mu \mathrm{g} / \mathrm{ml})$ : & $1.36 \mathrm{E}-01$ & $<8.00 \mathrm{E}-03$ & $\mathrm{Ti}$ \\
\hline & Uncorrected weight of impurity in sample $(\mu g)$ : & 6.66 & $<0.42$ & $<7.08$ \\
\hline $\mathrm{Ti}$ & Weight of impurity in blank $(\mu q)$ : & $<0.41$ & $<0.43$ & \\
\hline & Minimum corrected weight of impurity in sample $(\mu q)$ : & 6.25 & 0.00 & 6.25 \\
\hline & Maximum corrected weight of impurity in sample $(\mu g)$ : & 6.66 & 0.42 & 7.08 \\
\hline & Measured concentration of impurity in sample $(\mathrm{\mu g} / \mathrm{ml})$ : & $1.09 \mathrm{E}+00$ & $6.26 \mathrm{E}-03$ & v \\
\hline & Uncorrected weight of impurity in sample $(\mu \mathrm{g})$ : & 53.41 & 0.33 & 53.74 \\
\hline $\mathbf{v}$ & Weight of impurity in blank $(\mu g)$ : & $<0.10$ & $<0.11$ & \\
\hline & Minimum corrected weight of impurity in sample $(\mu \mathrm{g})$ : & 53.31 & 0.22 & 53.53 \\
\hline & Maximum corrected weight of impurity in sample $(\mu \mathrm{g})$ : & 53.41 & 0.33 & 53.74 \\
\hline
\end{tabular}

Comments

FCM Checked the official results of analyses for RMAL1910 and RMAL1918 on 7/10/2009

Fred c. Montgomen
$7-23-09$ 
Data Report Form DRF-26B: Measurement of SiC Burn-Leach Defects or Impurities by Burn-Leach

\begin{tabular}{|r|l|}
\hline Procedure: & AGR-CHAR-DAM-26 Rev. 1 \\
\hline Operator: & Fred Montgomery \\
\hline Compact lot ID: & LEU07-OP1-Z \\
\hline Compact lot description: & AGR-2 UCO Baseline Fuel, from G73J-14-93072A \\
\hline Compact ID numbers: & Burn-Leach Blank \\
\hline DRF filename: & IImc-agr\AGR\LeachBurnLeach \LEU07-OP1-Z_DRF26R1_01.xIs \\
\hline
\end{tabular}

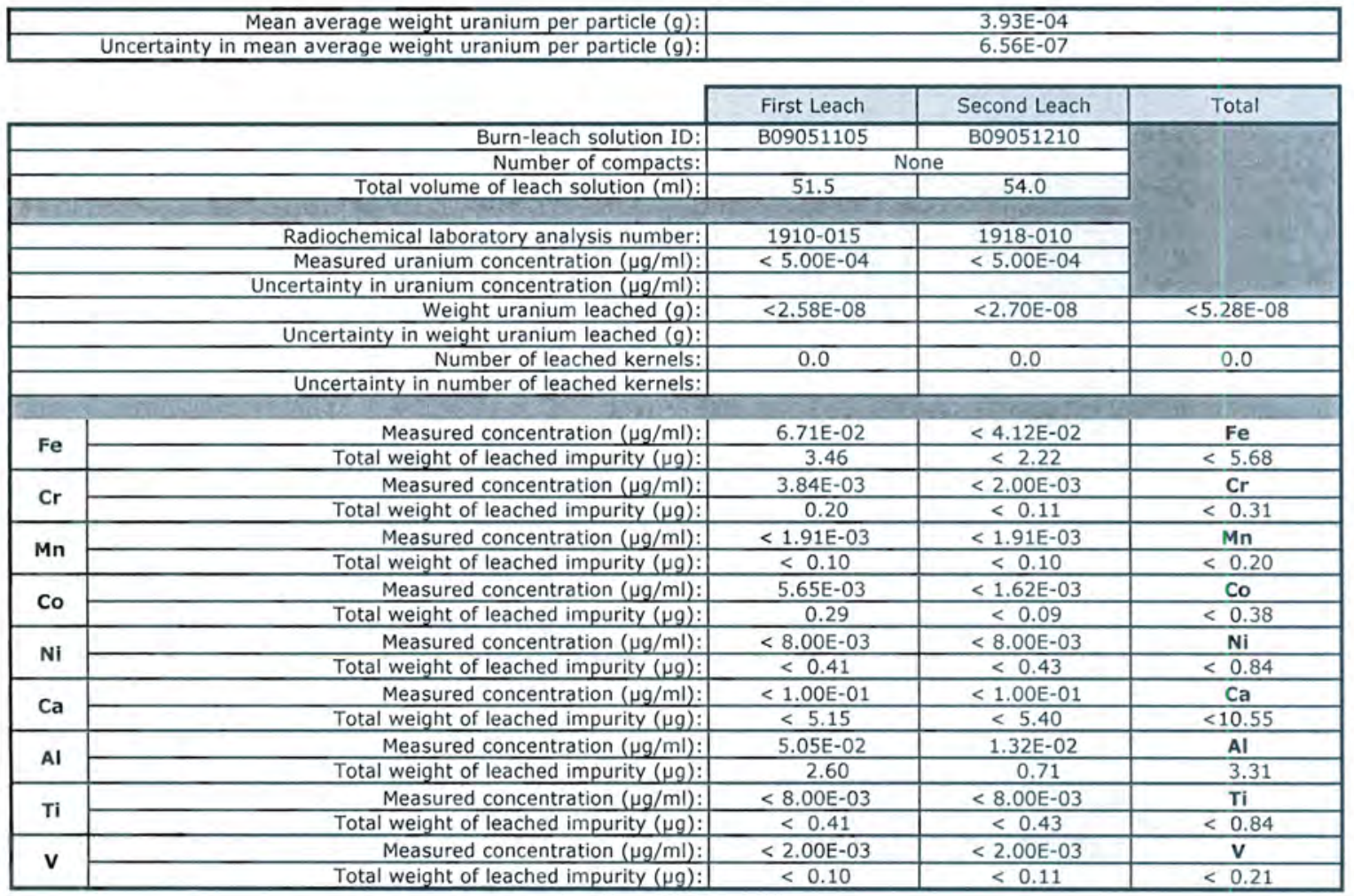

Comments

FCM Checked the official results of analyses for RMAL1910 and RMAL1918 on 7/10/2009

Fied c. Mont gomeey

$7-23-09$

$\frac{7-23-09}{\text { Date }}$


Data Report Form DRF-26A: Measurement of U Contamination or Impurities by Deconsolidation Leach

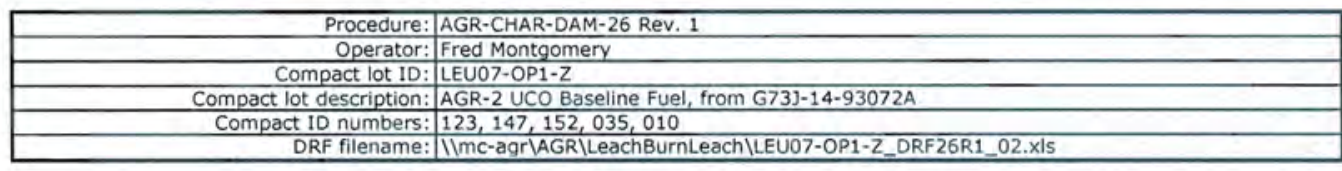

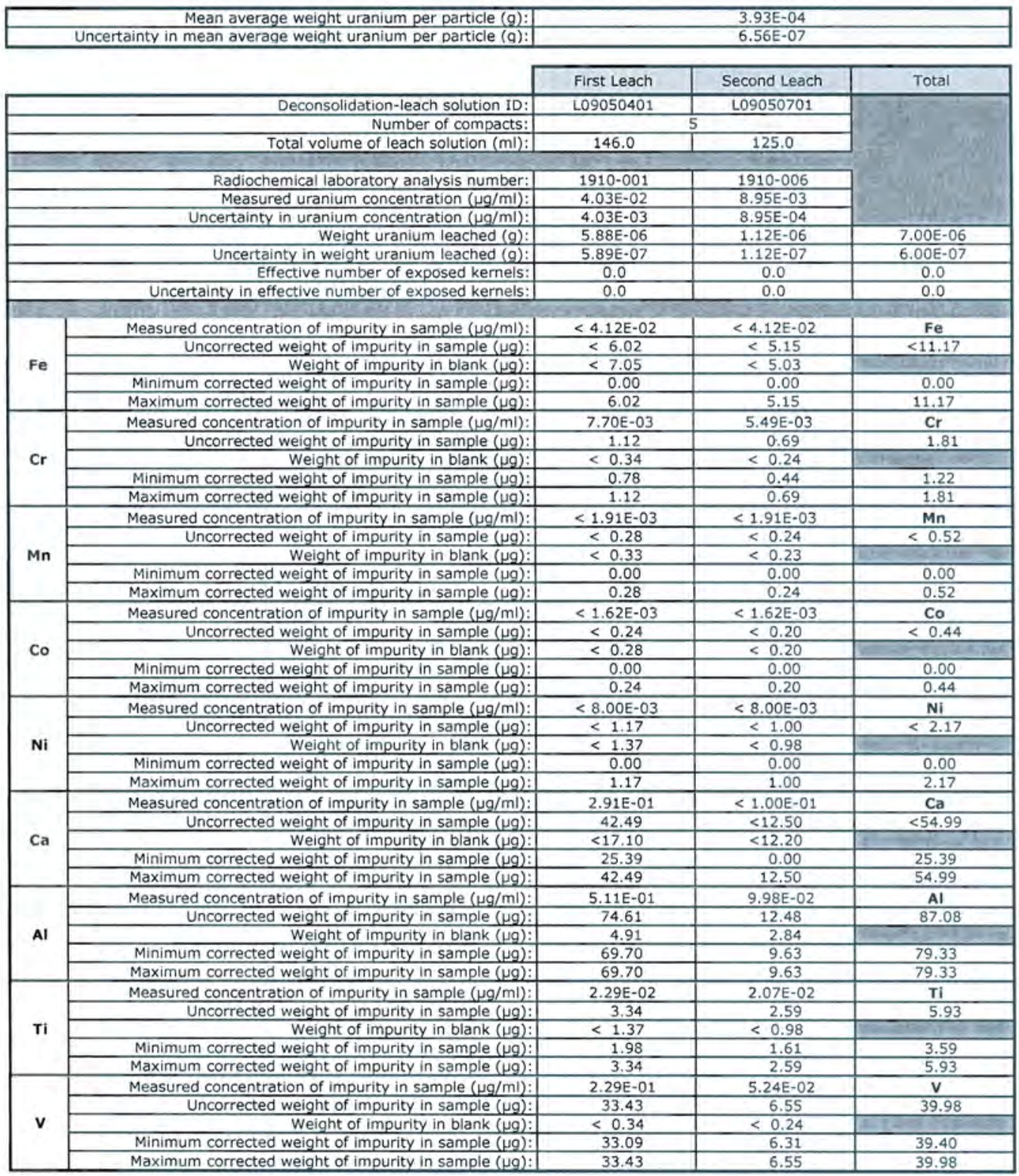

comments

Checked against official results of analyses for RMAL 1910 by FCM on 7/09/2009

Fed c. Monteromen

$7-23-09$ 
Data Report Form DRF-26A: Measurement of U Contamination or Impurities by Deconsolidation Leach

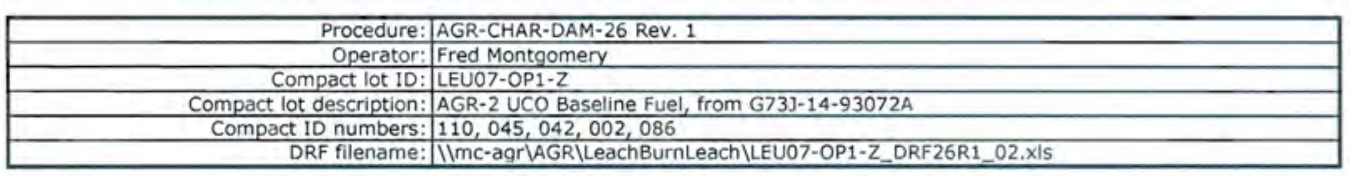

\begin{tabular}{|c|c|c|c|c|}
\hline \multirow{2}{*}{\multicolumn{2}{|c|}{$\begin{aligned} \text { Mean average weight uranium per particle }(\mathrm{g}): \\
\text { Uncertainty in mean average weight uranium per particle (q): } \\
\end{aligned}$}} & \multicolumn{3}{|c|}{$\begin{array}{l}3.93 \mathrm{E}-04 \\
6.56 \mathrm{E}-07 \\
\end{array}$} \\
\hline & & First Leach & Second Leach & Total \\
\hline & Deconsolidation-leach solution ID: & $L 09050402$ & $L 09050702$ & \\
\hline & Number of compacts: & & & \\
\hline & Total volume of leach solution (mi): & 146.0 & 129.0 & \\
\hline & Radiochemical laboratory analysis number: & $1910-002$ & $1910-007$ & \\
\hline & Measured uranium concentration $(\mathrm{\mu g} / \mathrm{ml})$ : & $5.18 \mathrm{E}+00$ & $2.65 \mathrm{E}-01$ & \\
\hline & Uncertainty in uranium concentration $(\mathrm{\mu g} / \mathrm{ml})$ : & $5.18 \mathrm{E}-01$ & $2.65 E-02$ & \\
\hline & Weight uranium leached $(9)$ : & $7.56 \mathrm{E}-04$ & $3.42 \mathrm{E}-05$ & $7.90 \mathrm{E}-04$ \\
\hline & Uncertainty in weight uranium leached $(g)$ : & $7.57 \mathrm{E}-05$ & $3.42 \mathrm{E}-06$ & $7.58 \mathrm{E}-05$ \\
\hline & Effective number of exposed kernels: & 1.9 & 0.1 & 2.0 \\
\hline & Uncertainty in effective number of exposed kernels: & 0.2 & 0.0 & 0.2 \\
\hline \multirow{5}{*}{$\mathrm{Fe}$} & Measured concentration of impurity in sample $(\mu \mathrm{g} / \mathrm{ml})$ : & $<4.12 \mathrm{E}-02$ & $<4.12 \mathrm{E}-02$ & $\mathrm{Fe}$ \\
\hline & Uncorrected weight of impurity in sample $(\mu g)$ : & $<6.02$ & $<5.31$ & $<11.33$ \\
\hline & Weight of impurity in blank $(\mu \mathrm{g})$ : & $<7.05$ & $<5.03$ & \\
\hline & Minimum corrected weight of impurity in sample $(\mu \mathrm{g})$ : & 0.00 & 0.00 & 0.00 \\
\hline & Maximum corrected weight of impurity in sample $(\mu \mathrm{g})$ : & 6.02 & 5.31 & 11.33 \\
\hline \multirow{5}{*}{$\mathrm{Cr}$} & Measured concentration of impurity in sample $(\mu \mathrm{g} / \mathrm{ml})$ : & $1.11 \mathrm{E}-02$ & $7.87 \mathrm{E}-03$ & Cr \\
\hline & Uncorrected weight of impurity in sample $(\mu \mathrm{g})$ : & 1.62 & 1.02 & 2.64 \\
\hline & Weight of impurity in blank $(\mu \mathrm{g})$ : & $<0.34$ & $<0.24$ & \\
\hline & Minimum corrected weight of impurity in sample $(\mu \mathrm{g})$ : & 1.28 & 0.77 & 2.05 \\
\hline & Maximum corrected weight of impurity in sample $(\mu \mathrm{g})$ : & 1.62 & 1.02 & 2.64 \\
\hline \multirow{4}{*}{$\mathrm{Mn}$} & Measured concentration of impurity in sample $(\mathrm{\mu g} / \mathrm{ml})$ : & $<1.91 \mathrm{E}-03$ & $<1.91 \mathrm{E}-03$ & $\mathrm{Mn}$ \\
\hline & Uncorrected weight of impurity in sample $(\mu \mathrm{g})$ : & $<0.28$ & $<0.25$ & $<0.53$ \\
\hline & Weight of impurity in blank $(\mu \mathrm{g})$ : & $<0.33$ & $<0.23$ & \\
\hline & $\begin{array}{l}\text { Minimum corrected weight of impurity in sample }(\mu g) \text { : } \\
\text { Maximum corrected weight of impurity in sample }(\mu g) \text { : }\end{array}$ & 0.00 & 0.00 & 0.00 \\
\hline \multirow{5}{*}{ co } & Measured concentration of impurity in sample $(\mu \mathrm{g} / \mathrm{ml})$ : & $<1.62 E-03$ & $<1.62 \mathrm{E}-03$ & Co \\
\hline & Uncorrected weight of impurity in sample $(\mu \mathrm{g})$ : & $<0.24$ & $<0.21$ & $<0.45$ \\
\hline & Weight of impurity in blank $(\mu \mathrm{g})$ : & $<0.28$ & $<0.20$ & \\
\hline & Minimum corrected weight of impurity in sample $(\mu \mathrm{g})$ : & 0.00 & 0.00 & 0.00 \\
\hline & Maximum corrected weight of impurity in sample $(\mu g)$ : & 0.24 & 0.21 & 0.45 \\
\hline \multirow{5}{*}{$\mathrm{Ni}$} & Measured concentration of impurity in sample $(\mu \mathrm{g} / \mathrm{ml})$ : & $<8.00 \mathrm{E}-03$ & $<8.00 \mathrm{E}-03$ & \\
\hline & Uncorrected weight of impurity in sample $(\mu \mathrm{g})$ : & $<1.17$ & $<1.03$ & $<2.20$ \\
\hline & $\begin{array}{c}\text { Weight of impurity in blank }(\mu g) \text { : } \\
\end{array}$ & $<1.37$ & $<0.98$ & \\
\hline & Minimum corrected weight of impurity in sample $(\mu g)$ : & 0.00 & 0.00 & 0.00 \\
\hline & Maximum corrected weight of impurity in sample $(\mu g)$ : & 1.17 & 1.03 & 2.20 \\
\hline \multirow{5}{*}{ Ca } & Measured concentration of impurity in sample $(\mu \mathrm{g} / \mathrm{ml})$ : & $4.10 \mathrm{E}-01$ & $2.78 \mathrm{E}-01$ & $\mathrm{Ca}$ \\
\hline & Uncorrected weight of impurity in sample $(\mu g)$ : & 59.86 & 35.86 & 95.72 \\
\hline & Weight of impurity in blank $(\mu g)$ : & $<17.10$ & $<12.20$ & \\
\hline & Minimum corrected weight of impurity in sample $(\mu g)$ : & 42.76 & 23.66 & 66.42 \\
\hline & Maximum corrected weight of impurity in sample $(\mu \mathrm{g})$ : & 59.86 & 35.86 & 95.72 \\
\hline \multirow{5}{*}{ Al } & Measured concentration of impurity in sample $(\mu \mathrm{g} / \mathrm{ml})$ : & $4.21 \mathrm{E}-01$ & $1.22 \mathrm{E}-01$ & Al \\
\hline & Uncorrected weight of impurity in sample $(\mu \mathrm{g})$ : & 61.47 & 15.74 & 77.20 \\
\hline & Weight of impurity in blank $(\mu \mathrm{g})$ : & 4.91 & 2.84 & \\
\hline & Minimum corrected weight of impurity in sample $(\mu g)$ : & 56.56 & 12.90 & 69.45 \\
\hline & Maximum corrected weight of impurity in sample $(\mu \mathrm{g})$ : & 56.56 & 12.90 & 69.45 \\
\hline \multirow{5}{*}{$\mathrm{Ti}$} & Measured concentration of impurity in sample $(\mu \mathrm{g} / \mathrm{ml})$ : & 3.07E-02 & $2.88 \mathrm{E}-02$ & $\mathrm{Ti}$ \\
\hline & Uncorrected weight of impurity in sample $(\mu g)$ : & 4.48 & 3.72 & 8.20 \\
\hline & Weight of impurity in blank $(\mu \mathrm{\mu g})$ : & $<1.37$ & $<0.98$ & 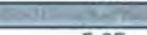 \\
\hline & Minimum corrected weight of impurity in sample $(\mu \mathrm{g})$ : & 3.11 & 2.74 & 5.85 \\
\hline & Maximum corrected weight of impurity in sample $(\mu g)$ : & 4.48 & 3.72 & 8.20 \\
\hline \multirow{5}{*}{$\mathbf{v}$} & Measured concentration of impurity in sample $(\mu \mathrm{g} / \mathrm{ml})$ : & $2.34 \mathrm{E}-01$ & $6.14 \mathrm{E}-02$ & $\mathrm{v}$ \\
\hline & Uncorrected weight of impurity in sample $(\mu g)$ : & 34.16 & 7.92 & 42.08 \\
\hline & Weight of impurity in blank $(\mu g)$ : & $<0.34$ & $<0.24$ & 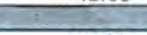 \\
\hline & Minimum corrected weight of impurity in sample $(\mu g)$ : & 33.82 & 7.68 & 41.50 \\
\hline & Maximum corrected weight of impurity in sample $(\mu g)$ : & 34.16 & 7.92 & 42.08 \\
\hline
\end{tabular}

Comments

Checked against official results of analyses for RMAL1910 by FCM on 7/09/2009

Fied c. monteomey

$7-23-09$ 
Data Report Form DRF-26A: Measurement of U Contamination or Impurities by Deconsolidation Leach

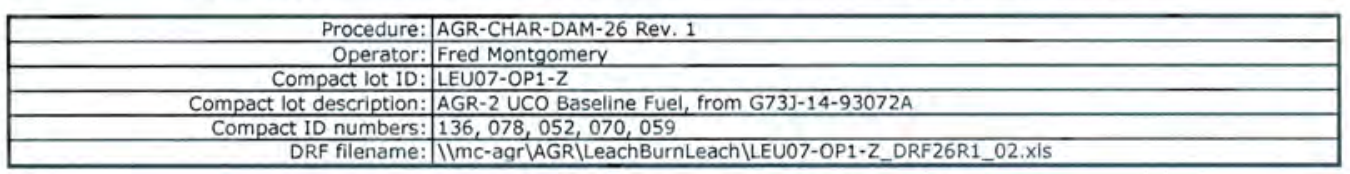

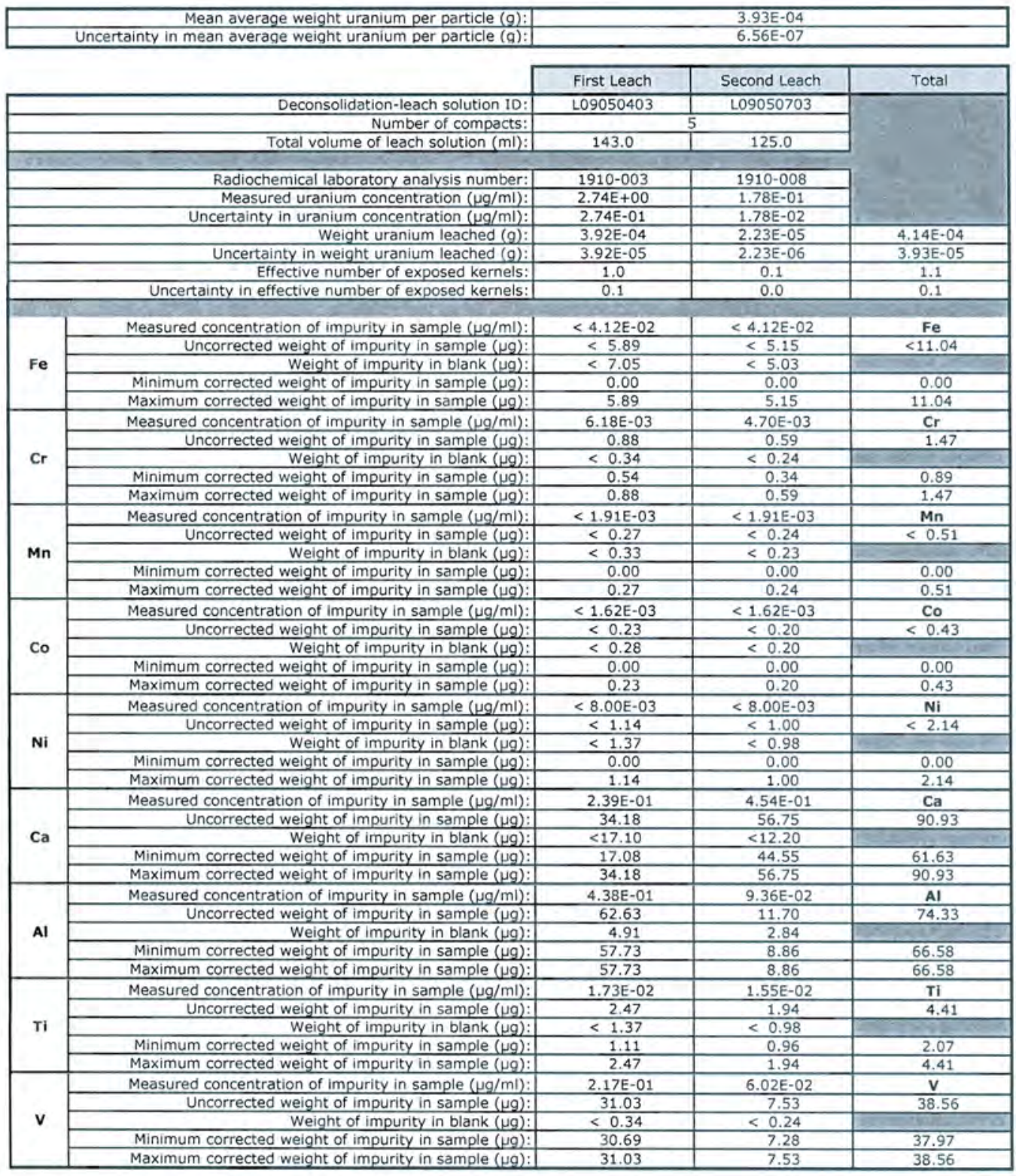

Comments

Checked against official results of analyses for RMAL1910 by FCM on 7/09/2009

\section{Fied C. Montsomey 7-23-09}


Data Report Form DRF-26A: Measurement of U Contamination or Impurities by Deconsolidation Leach

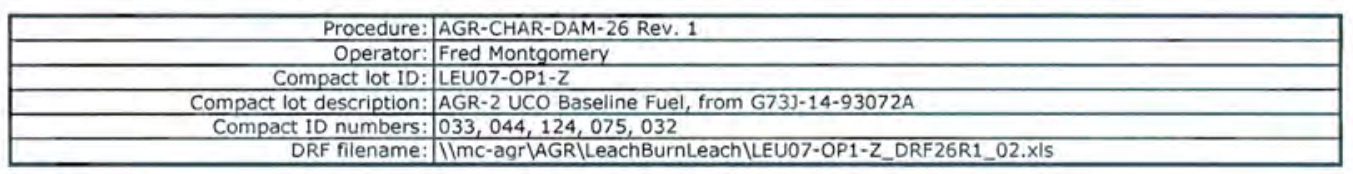

\begin{tabular}{|c|c|c|c|c|}
\hline \multicolumn{2}{|r|}{$\begin{array}{r}\text { Mean average weight uranium per particle }(g): \\
\text { Uncertainty in mean average weight uranium per particle }(q):\end{array}$} & \multicolumn{3}{|c|}{$\frac{3.93 E-04}{6.56 E-07}$} \\
\hline & & First Leach & Second Leach & Total \\
\hline & Deconsolidation-leach solution ID: & $L 09050404$ & L09050704 & \\
\hline & Number of compacts: & & & \\
\hline & Total volume of leach solution $(\mathrm{ml})$ : & 144.0 & 126.0 & \\
\hline & Radiochemical laboratory analysis number: & & & \\
\hline & $\begin{array}{l}\text { Radiochemical laboratory analysis number: } \\
\text { Measured uranium concentration }(\mu \mathrm{g} / \mathrm{ml}) \text { : }\end{array}$ & $\frac{1910-004}{4.43 \mathrm{E}-01}$ & $\frac{1910-009}{1.71 E-02}$ & \\
\hline & Uncertainty in uranium concentration $(\mu \mathrm{g} / \mathrm{ml})$ : & $4.43 E-02$ & $1.71 \mathrm{E}-03$ & \\
\hline & Weight uranium leached $(g)$ : & $6.38 \mathrm{E}-05$ & $2.15 \mathrm{E}-06$ & $6.59 \mathrm{E}-05$ \\
\hline & Uncertainty in weight uranium leached $(g)$ : & $6.39 \mathrm{E}-06$ & $2.16 \mathrm{E}-07$ & $6.39 \mathrm{E}-06$ \\
\hline & Effective number of exposed kernels: & 0.2 & 0.0 & 0.2 \\
\hline & Uncertainty in effective number of exposed kernels: & 0.0 & 0.0 & 0.0 \\
\hline \multirow{5}{*}{ Fe } & Measured concentration of impurity in sample $(\mu \mathrm{g} / \mathrm{ml})$ : & $<4.12 \mathrm{E}-02$ & $<4.12 \mathrm{E}-02$ & $\mathrm{Fe}$ \\
\hline & Uncorrected weight of impurity in sample $(\mu g)$ : & $<5.93$ & $<5.19$ & $<11.12$ \\
\hline & Weight of impurity in blank $(\mu \mathrm{g})$ : & $<7.05$ & $<5.03$ & \\
\hline & Minimum corrected weight of impurity in sample $(\mu g)$ : & 0.00 & 0.00 & 0.00 \\
\hline & Maximum corrected weight of impurity in sample $(\mu g)$ : & 5.93 & 5.19 & 11.12 \\
\hline \multirow{5}{*}{$\mathrm{Cr}$} & Measured concentration of impurity in sample $(\mu \mathrm{g} / \mathrm{ml})$ : & $1.25 \mathrm{E}-02$ & $8.49 \mathrm{E}-03$ & $\mathrm{Cr}$ \\
\hline & Uncorrected weight of impurity in sample $(\mu g)$ : & 1.80 & 1.07 & 2.87 \\
\hline & Weight of impurity in blank $(\mu g)$ : & $<0.34$ & $<0.24$ & \\
\hline & Minimum corrected weight of impurity in sample $(\mu g)$ : & 1.46 & 0.83 & 2.28 \\
\hline & Maximum corrected weight of impurity in sample $(\mu \mathrm{g})$ : & 1.80 & 1.07 & 2.87 \\
\hline \multirow{5}{*}{ Mn } & Measured concentration of impurity in sample $(\mu \mathrm{g} / \mathrm{ml})$ : & $<1.93 \mathrm{E}-03$ & $<1.91 \mathrm{E}-03$ & $\mathrm{Mn}$ \\
\hline & Uncorrected weight of impurity in sample $(\mu \mathrm{g})$ : & $<0.28$ & $<0.24$ & $<0.52$ \\
\hline & Weight of impurity in blank $(\mu g)$ : & $<0.33$ & $<0.23$ & \\
\hline & Minimum corrected weight of impurity in sample $(\mu g)$ : & 0.00 & 0.00 & 0.00 \\
\hline & Maximum corrected weight of impurity in sample $(\mu \mathrm{g})$ : & 0.28 & 0.24 & 0.52 \\
\hline \multirow{5}{*}{ co } & Measured concentration of impurity in sample $(\mu \mathrm{g} / \mathrm{ml})$ : & $<1.62 \mathrm{E}-03$ & $<1.62 \mathrm{E}-03$ & Co \\
\hline & Uncorrected weight of impurity in sample $(\mu \mathrm{g})$ : & $<0.23$ & $<0.20$ & $<0.44$ \\
\hline & Weight of impurity in blank $(\mu g)$ : & $<0.28$ & $<0.20$ & \\
\hline & Minimum corrected weight of impurity in sample $(\mu g)$ : & 0.00 & 0.00 & 0.00 \\
\hline & Maximum corrected weight of impurity in sample $(\mu g)$ : & 0.23 & 0.20 & 0.44 \\
\hline \multirow{5}{*}{$\mathrm{Ni}$} & Measured concentration of impurity in sample $(\mu \mathrm{g} / \mathrm{ml})$ : & $<8.00 \mathrm{E}-03$ & $<8.00 \mathrm{E}-03$ & $\mathbf{N i}$ \\
\hline & Uncorrected weight of impurity in sample $(\mu g)$ : & $<1.15$ & $<1.01$ & $<2.16$ \\
\hline & Weight of impurity in blank $(\mu \mathrm{g})$ : & $<1.37$ & $<0.98$ & \\
\hline & Minimum corrected weight of impurity in sample $(\mu \mathrm{g})$ : & 0.00 & 0.00 & 0.00 \\
\hline & Maximum corrected weight of impurity in sample $(\mu \mathrm{g})$ : & 1.15 & 1.01 & 2.16 \\
\hline \multirow{5}{*}{$\mathrm{Ca}$} & Measured concentration of impurity in sample $(\mu \mathrm{g} / \mathrm{ml})$ : & $9.47 \mathrm{E}-01$ & $3.54 \mathrm{E}-01$ & $\mathrm{Ca}$ \\
\hline & Uncorrected weight of impurity in sample $(\mu \mathrm{g})$ : & 136.37 & 44.60 & 180.97 \\
\hline & Weight of impurity in blank $(\mathrm{\mu g})$ : & $<17.10$ & $<12.20$ & \\
\hline & Minimum corrected weight of impurity in sample $(\mu \mathrm{g})$ : & 119.27 & 32.40 & 151.67 \\
\hline & Maximum corrected weight of impurity in sample $(\mu \mathrm{g})$ : & 136.37 & 44.60 & 180.97 \\
\hline \multirow{5}{*}{ Al } & Measured concentration of impurity in sample $(\mu \mathrm{g} / \mathrm{ml})$ : & $5.08 \mathrm{E}-01$ & $1.95 \mathrm{E}-01$ & Al \\
\hline & Uncorrected weight of impurity in sample $(\mu \mathrm{g})$ : & 73.15 & 24.57 & 97.72 \\
\hline & Weight of impurity in blank $(\mu \mathrm{g})$ : & 4.91 & 2.84 & \\
\hline & Minimum corrected weight of impurity in sample $(\mu \mathrm{g})$ : & 68.24 & 21.73 & 89.97 \\
\hline & Maximum corrected weight of impurity in sample $(\mathrm{ug})$ : & 68.24 & 21.73 & 89.97 \\
\hline \multirow{5}{*}{$\mathrm{Ti}$} & Measured concentration of impurity in sample $(\mu \mathrm{g} / \mathrm{ml})$ : & $3.19 \mathrm{E}-02$ & $3.03 \mathrm{E}-02$ & $\mathbf{T i}$ \\
\hline & Uncorrected weight of impurity in sample $(\mu \mathrm{g})$ : & 4.59 & 3.82 & 8.41 \\
\hline & Weight of impurity in blank $(\mu \mathrm{g})$ : & $<1.37$ & $<0.98$ & \\
\hline & Minimum corrected weight of impurity in sample $(\mu \mathrm{g})$ : & 3.23 & 2.84 & 6.07 \\
\hline & Maximum corrected weight of impurity in sample $(\mu \mathrm{g})$ : & 4.59 & 3.82 & 8.41 \\
\hline \multirow{5}{*}{$\mathbf{v}$} & Measured concentration of impurity in sample $(\mu \mathrm{g} / \mathrm{ml})$ : & $2.35 \mathrm{E}-01$ & $6.61 \mathrm{E}-02$ & v \\
\hline & Uncorrected weight of impurity in sample $(\mu \mathrm{g})$ : & 33.84 & 8.33 & 42,17 \\
\hline & Weight of impurity in blank $(\mathrm{g} g)$ : & $<0.34$ & $<0.24$ & \\
\hline & Minimum corrected weight of impurity in sample $(\mu \mathrm{g})$ : & 33.50 & 8.08 & 41.58 \\
\hline & Maximum corrected weight of impurity in sample $(\mu \mathrm{g})$ : & 33.84 & 8.33 & 42.17 \\
\hline
\end{tabular}

Comments

Checked against official results of analyses for RMAL1910 by FCM on 7/09/2009

7wel c. Montigomey


Data Report Form DRF-26A: Measurement of U Contamination or Impurities by Deconsolidation Leach

\begin{tabular}{|c|c|}
\hline Procedure: & AGR-CHAR-DAM-26 Rev. 1 \\
\hline Operator: & Fred Montgomery \\
\hline Compact lot ID: & LEU07-OP1-Z \\
\hline Compact lot description: & AGR-2 UCO Baseline Fuel, from G73J-14-93072A \\
\hline Compact ID numbers: & Deconsolidation Leach Blank \\
\hline DRF filename: & 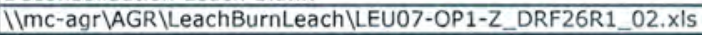 \\
\hline
\end{tabular}

\begin{tabular}{|c|c|c|c|c|}
\hline & Mean average weight uranium per particle $(\mathrm{g})$ : & & $3.93 \mathrm{E}-04$ & \\
\hline & Uncertainty in mean average weight uranium per particle $(\mathrm{g})$ : & & $6.56 \mathrm{E}-07$ & \\
\hline & & First Leach & Second Leach & Total \\
\hline & Deconsolidation-leach solution ID: & L09050405 & L09050705 & \\
\hline & Number of compacts: & & & \\
\hline & Total volume of leach solution $(\mathrm{ml})$ : & 171.0 & 122.0 & \\
\hline & 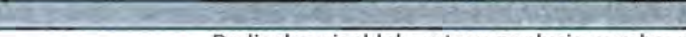 & & & \\
\hline & Radiochemical laboratory analysis number: & $1910-005$ & $1910-010$ & \\
\hline & $\begin{array}{l}\text { Measured uranium concentration }(\mu \mathrm{g} / \mathrm{ml}) \text { : } \\
\text { Uncertainty in uranium concentration }(\mu \mathrm{g} / \mathrm{ml}) \text { : }\end{array}$ & $<5.00 \mathrm{E}-04$ & $<5.00 \mathrm{E}-04$ & \\
\hline & $\begin{array}{r}\text { Uncertainty in uranium concentration }(\mu \mathrm{g} / \mathrm{ml}) \text { : } \\
\text { Weight uranium leached }(\mathrm{g}) \text { : }\end{array}$ & $<8.55 \mathrm{E}-08$ & $<6.10 \mathrm{E}-08$ & $<1.47 \mathrm{E}-07$ \\
\hline & Uncertainty in weight uranium leached $(\mathrm{g})$ : & & & \\
\hline & Effective number of exposed kernels: & 0.0 & 0.0 & 0.0 \\
\hline & Uncertainty in effective number of exposed kernels: & & & \\
\hline & Measured concentration $(\mu \mathrm{g} / \mathrm{ml})$ : & $<4.12 \mathrm{E}-02$ & $<4.12 \mathrm{E}-02$ & $\mathbf{F e}$ \\
\hline $\mathrm{Fe}$ & Total weight of leached impurity $(\mu \mathrm{g})$ : & $<7.05$ & $<5.03$ & $<12.07$ \\
\hline & Measured concentration $(\mu \mathrm{g} / \mathrm{ml})$ : & $<2.00 \mathrm{E}-03$ & $<2.00 \mathrm{E}-03$ & $\mathrm{Cr}$ \\
\hline $\mathrm{Cr}$ & Total weight of leached impurity $(\mu \mathrm{g})$ : & $<0.34$ & $<0.24$ & $<0.59$ \\
\hline Mn & Measured concentration $(\mu \mathrm{g} / \mathrm{ml})$ : & $<1.91 \mathrm{E}-03$ & $<1.91 \mathrm{E}-03$ & $\mathrm{Mn}$ \\
\hline $\mathrm{Mn}$ & Total weight of leached impurity $(\mu \mathrm{g})$ : & $<0.33$ & $<0.23$ & $<0.56$ \\
\hline Co & Measured concentration $(\mu \mathrm{g} / \mathrm{ml})$ : & $<1.62 \mathrm{E}-03$ & $<1.62 \mathrm{E}-03$ & Co \\
\hline co & Total weight of leached impurity $(\mu \mathrm{g})$ : & $<0.28$ & $<0.20$ & $<0.47$ \\
\hline & Measured concentration $(\mu \mathrm{g} / \mathrm{ml})$ : & $<8.00 \mathrm{E}-03$ & $<8.00 \mathrm{E}-03$ & $\mathrm{Ni}$ \\
\hline NI & Total weight of leached impurity $(\mu \mathrm{g})$ : & $<1.37$ & $<0.98$ & $<2.34$ \\
\hline Ca & Measured concentration $(\mu \mathrm{g} / \mathrm{ml})$ : & $<1.00 \mathrm{E}-01$ & $<1.00 \mathrm{E}-01$ & $\mathrm{Ca}$ \\
\hline $\mathrm{Ca}$ & Total weight of leached impurity $(\mu \mathrm{g})$ : & $<17.10$ & $<12.20$ & $<29.30$ \\
\hline & Measured concentration $(\mu \mathrm{g} / \mathrm{ml})$ : & $2.87 \mathrm{E}-02$ & $2.33 \mathrm{E}-02$ & Al \\
\hline AI & Total weight of leached impurity $(\mu \mathrm{g})$ : & 4.91 & 2.84 & 7.75 \\
\hline & Measured concentration $(\mu \mathrm{g} / \mathrm{ml})$ : & $<8.00 \mathrm{E}-03$ & $<8.00 \mathrm{E}-03$ & $\mathrm{Ti}$ \\
\hline $\mathrm{TI}$ & Total weight of leached impurity $(\mu \mathrm{g})$ : & $<1.37$ & $<0.98$ & $<2.34$ \\
\hline$v$ & Measured concentration $(\mu \mathrm{g} / \mathrm{ml})$ : & $<2.00 \mathrm{E}-03$ & $<2.00 \mathrm{E}-03$ & v \\
\hline$v$ & Total weight of leached impurity $(\mu \mathrm{g})$ : & $<0.34$ & $<0.24$ & $<0.59$ \\
\hline
\end{tabular}

Comments

Checked against official results of analyses for RMAL1910 by FCM on 7/09/2009

\section{Fied C. Montgomeuy}

$2-23-09$ 
Data Report Form DRF-26A: Measurement of U Contamination or Impurities by Deconsolidation Leach

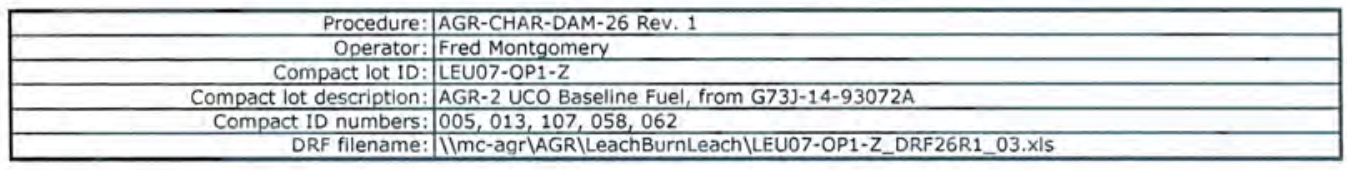

\begin{tabular}{|c|c|c|c|c|}
\hline \multirow{2}{*}{\multicolumn{2}{|c|}{$\begin{array}{r}\text { Mean average weight uranium per particle }(g): \\
\text { Uncertainty in mean average weight uranium per particle }(a): \\
\end{array}$}} & \multicolumn{3}{|c|}{$\begin{array}{l}3.93 \mathrm{E}-04 \\
6.56 \mathrm{E}-07 \\
\end{array}$} \\
\hline & & First Leach & Second Leach & Total \\
\hline & Deconsolidation-leach solution ID: & L09060201 & L09060401 & \\
\hline & Number of compacts: & & & \\
\hline & Total volume of leach solution $(\mathrm{ml})$ : & 137.0 & 109.0 & \\
\hline & Radiochemical laboratory analysis number: & $1999-001$ & $1999-006$ & \\
\hline & Measured uranium concentration $(\mu \mathrm{g} / \mathrm{ml})$ : & $5.50 E+00$ & $4.15 E-01$ & \\
\hline & Uncertainty in uranlum concentration $(\mathrm{\mu g} / \mathrm{ml})$ : & $5.50 \mathrm{E}-01$ & $4.15 E-02$ & \\
\hline & Weight uranium leached $(g)$ : & $7.54 \mathrm{E}-04$ & $4.52 \mathrm{E}-0.5$ & $7.99 \mathrm{E}-04$ \\
\hline & Uncertainty in weight uranium leached $(\mathrm{g})$ : & $7.54 E-05$ & $4.53 E-06$ & $7.56 \mathrm{E} \cdot 05$ \\
\hline & Effective number of exposed kernels: & 1.9 & 0.1 & 2.0 \\
\hline & Uncertainty in effective number of exposed kernels: & 0.2 & 0.0 & 0.2 \\
\hline \multirow{5}{*}{$\mathrm{Fe}$} & Measured concentration of impurity in sample $(\mathrm{\mu g} / \mathrm{ml})$ : & $<4.12 \mathrm{E}-02$ & $<4,12 \mathrm{E}-02$ & \\
\hline & Uncorrected weight of impurity in sample $(\mu g)$ : & $<5.64$ & $<4.49$ & $\frac{\mathrm{re}}{<10.14}$ \\
\hline & Weight of impurity in blank $(\mu g)$ : & $<6.72$ & $<4.86$ & \\
\hline & Minimum corrected weight of impurity in sample $(\mu \mathrm{g})$ : & 0.00 & 0.00 & 0.00 \\
\hline & Maximum corrected weight of impurity in sample $(\mu g)$ : & 5.64 & 4.49 & 10.14 \\
\hline \multirow{5}{*}{$\mathrm{Cr}$} & Measured concentration of impurity in sample $(\mathrm{\mu g} / \mathrm{ml})$ : & $8.74 E-03$ & $9.26 \mathrm{E}-03$ & $\mathrm{Cr}$ \\
\hline & Uncorrected weight of impurity in sample $(\mu \mathrm{g})$ : & 1.20 & 1.01 & 2.21 \\
\hline & Weight of impurity in blank $(\mu \mathrm{g})$ : & $<0.33$ & $<0.24$ & sene \\
\hline & Minimum corrected weight of impurity in sample $(\mu g)$ : & 0.87 & 0.77 & 1.64 \\
\hline & Maximum corrected weight of impurity in sample $(\mu \mathrm{g})$ : & 1.20 & 1.01 & 2.21 \\
\hline \multirow{5}{*}{$\mathrm{Mn}$} & Measured concentration of impurity in sample $(\mu \mathrm{g} / \mathrm{ml})$ : & $<1.91 \mathrm{E}-03$ & $<1.91 \mathrm{E}-03$ & $\mathrm{Mn}$ \\
\hline & Uncorrected weight of impurity in sample $(\mu \mathrm{g})$ : & $<0.26$ & $\leq 0.21$ & $<0.47$ \\
\hline & Weight of impurity in blank $(\mu \mathrm{g})$ : & $<0.31$ & $<0.23$ & \\
\hline & Minimum corrected weight of impurity in sample $(\mu g)$ : & 0.00 & 0.00 & 0.00 \\
\hline & Maximum corrected weight of impurity in sample $(\mu \mathrm{g})$ : & 0.26 & 0.21 & 0.47 \\
\hline \multirow{5}{*}{ co } & Measured concentration of impurity in sample $(\mu \mathrm{g} / \mathrm{ml})$ : & $<1.62 \mathrm{E}-03$ & $<1.62 \mathrm{E}-03$ & Co \\
\hline & Uncorrected weight of impurity in sample $(\mu \mathrm{g})$ : & $<0.22$ & $<0.18$ & $<0.40$ \\
\hline & Weight of impurity in blank $(\mu \mathrm{g})$ : & $<0.26$ & $<0.19$ & \\
\hline & Minimum corrected weight of impurity in sample $(\mu g)$ : & 0.00 & 0.00 & 0.00 \\
\hline & Maximum corrected weight of impurity in sample $(\mu \mathrm{g})$ : & 0.22 & 0.18 & 0.40 \\
\hline \multirow{5}{*}{$\mathrm{Ni}$} & Measured concentration of impurity in sample $(\mathrm{\mu g} / \mathrm{ml})$ : & $<8.00 \mathrm{E}-03$ & $<8.00 \mathrm{E}-03$ & $\mathrm{Ni}$ \\
\hline & Uncorrected weight of impurity in sample $(\mu \mathrm{g})$ : & $<1.10$ & $<0.87$ & $<1.97$ \\
\hline & Weight of impurity in blank $(\mu \mathrm{g})$ : & $<1.30$ & $<0.94$ & \\
\hline & Minimum corrected weight of impurity in sample $(\mu \mathrm{g})$ : & 0.00 & 0.00 & 0.00 \\
\hline & Maximum corrected weight of impurity in sample $(\mu g)$ : & 1.10 & 0.87 & 1.97 \\
\hline \multirow{5}{*}{ Ca } & Measured concentration of impurity in sample $(\mu \mathrm{g} / \mathrm{ml})$ : & $<1.00 \mathrm{E}-01$ & $<1.00 \mathrm{E}-01$ & $\mathrm{Ca}$ \\
\hline & Uncorrected weight of impurity in sample $(\mu \mathrm{g})$ : & $<13.70$ & $<10.90$ & $<24.60$ \\
\hline & Weight of impurity in blank $(\mathrm{pg})$ : & $<16.30$ & $<11.80$ & \\
\hline & Minimum corrected weight of impurity in sample $(\mu \mathrm{g})$ : & 0.00 & 0.00 & 0.00 \\
\hline & Maximum corrected weight of impurity in sample $(\mu g)$ : & 13.70 & 10.90 & 24.60 \\
\hline \multirow{5}{*}{ Al } & Measured concentration of impurity in sample $(\mathrm{\mu g} / \mathrm{ml})$ : & $1.70 E+00$ & $1.64 \mathrm{E}-01$ & Al \\
\hline & Uncorrected weight of impurity in sample $(\mu \mathrm{g})$ : & 232.90 & 17.88 & 250.78 \\
\hline & Weight of impurity in blank $(\mu \mathrm{g})$ : & 11.70 & 3.95 & 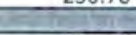 \\
\hline & Minimum corrected weight of impurity in sample $(\mu \mathrm{g})$ : & 221.20 & 13.92 & 235.12 \\
\hline & Maximum corrected weight of impurity in sample $(\mu g)$ : & 221.20 & 13.92 & 235.12 \\
\hline \multirow{5}{*}{$\mathrm{Ti}$} & Measured concentration of impurity in sample $(\mathrm{\mu g} / \mathrm{ml})$ : & $2.40 \mathrm{E}-02$ & $3.18 \mathrm{E}-02$ & Ti \\
\hline & Uncorrected weight of impurity in sample $(\mu g)$ : & 3.29 & 3.47 & 6.75 \\
\hline & Weight of impurity in blank $(\mu g)$ : & $<1.30$ & $<0.94$ & \\
\hline & Minimum corrected weight of impurity in sample $(\mu \mathrm{g})$ : & 1.98 & 2.52 & 4.51 \\
\hline & Maximum corrected weight of impurity in sample $(\mu \mathrm{g})$ : & 3.29 & 3.47 & 6.75 \\
\hline \multirow{5}{*}{ v } & Measured concentration of impurity in sample $(\mathrm{\mu g} / \mathrm{ml})$ : & $2.16 \mathrm{E}-01$ & $7.76 \mathrm{E}-02$ & $\mathrm{v}$ \\
\hline & Uncorrected weight of impurity in sample $(\mu \mathrm{g})$ : & 29.59 & 8.46 & 38.05 \\
\hline & Weight of impurity in blank $(\mu \mathrm{g})$ : & $<0.33$ & $<0.24$ & \\
\hline & Minimum corrected weight of impurity in sample $(\mu \mathrm{g})$ : & 29.27 & 8.22 & 37.49 \\
\hline & Maximum corrected weight of impurity in sample $(\mu \mathrm{g})$ : & 29.59 & 8.46 & 38.05 \\
\hline
\end{tabular}

Comments

Checked against official results of RMAL1999 by FCM on 7/14/2009

Fred c. Montgomerey
$7-23-09$ 
Data Report Form DRF-26A: Measurement of U Contamination or Impurities by Deconsolidation Leach

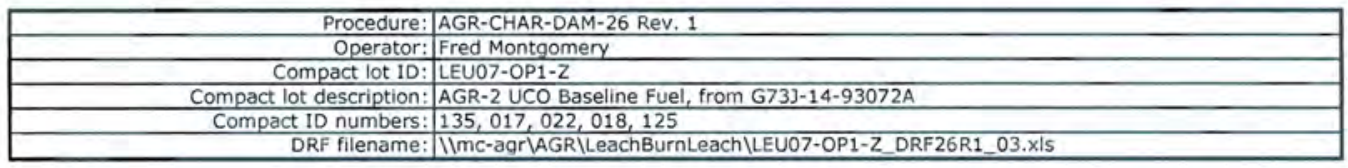

\begin{tabular}{|c|c|c|c|c|}
\hline & $\begin{array}{l}\text { Mean average weight uranium per particle }(g) \text { : } \\
\text { Uncertainty in mean average weight uranium per particle }(q) \text { : }\end{array}$ & & $\begin{array}{l}3.93 E-04 \\
6.56 E-07 \\
\end{array}$ & \\
\hline & & First Leach & Second Leach & Total \\
\hline & Deconsolidation-leach solution ID: & L09060202 & L09060402 & \\
\hline & Number of compacts: & & & \\
\hline & Total volume of leach solution $(\mathrm{ml})$ : & 139.0 & 112.0 & \\
\hline & 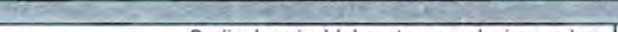 & & & \\
\hline & Radiochemical laboratory analysis number: & $1999-002$ & $1999-007$ & \\
\hline & Measured uranium concentration $(\mu \mathrm{g} / \mathrm{ml})$ : & $5.87 \mathrm{E}-02$ & $1.98 \mathrm{E}-02$ & \\
\hline & Uncertainty in uranium concentration $(\mu \mathrm{g} / \mathrm{ml})$ : & $5.87 E-03$ & $1.98 \mathrm{E}-03$ & \\
\hline & Weight uranium leached $(g)$ : & $8.16 \mathrm{E}-06$ & $2.22 \mathrm{E}-06$ & $1.04 \mathrm{E}-05$ \\
\hline & Uncertainty in weight uranium leached $(g)$ : & $8.17 E-07$ & $2.22 \mathrm{E}-07$ & $8.46 \mathrm{E}-07$ \\
\hline & Effective number of exposed kernels: & 0.0 & 0.0 & 0.0 \\
\hline & Uncertainty in effective number of exposed kernels: & 0.0 & 0.0 & 0.0 \\
\hline & Measured concentration of impurity in sample $(\mu \mathrm{g} / \mathrm{ml})$ : & & & \\
\hline & Uncorrected weight of impurity in sample $(\mu \mathrm{g})$ : & $\frac{4.12 L^{-02}}{<5.73}$ & $<4.61$ & $\frac{r e}{<10.34}$ \\
\hline $\mathrm{Fe}$ & Weight of impurity in blank $(\mu g)$ : & $<6.72$ & $<4.86$ & \\
\hline & Minimum corrected weight of impurity in sample $(\mu g)$ : & 0.00 & 0.00 & 0.00 \\
\hline & Maximum corrected weight of impurity in sample $(\mu \mathrm{g})$ : & 5.73 & 4.61 & 10.34 \\
\hline & Measured concentration of impurity in sample $(\mu \mathrm{g} / \mathrm{ml})$ : & 1.27E-02 & $8.44 \mathrm{E}-03$ & \\
\hline & Uncorrected weight of impurity in sample $(\mu \mathrm{g})$ : & 1.77 & 0.95 & 2.71 \\
\hline $\mathrm{Cr}$ & Weight of impurity in blank $(\mu \mathrm{g})$ : & $<0.33$ & $<0.24$ & \\
\hline & Minimum corrected weight of impurity in sample $(\mu \mathrm{g})$ : & 1.44 & 0.71 & 2.15 \\
\hline & Maximum corrected weight of impurity in sample $(\mu g)$ : & 1.77 & 0.95 & 2.71 \\
\hline & Measured concentration of impurity in sample $(\mathrm{\mu g} / \mathrm{ml})$ : & $<1.91 \mathrm{E}-03$ & $<1.91 \mathrm{E}-03$ & $\mathrm{Mn}$ \\
\hline & Uncorrected weight of impurity in sample $(\mu g)$ : & $<0.27$ & $<0.21$ & $<0.48$ \\
\hline Mn & Weight of impurity in blank $(\mathrm{g} g)$ : & $<0.31$ & $<0.23$ & \\
\hline & Minimum corrected weight of impurity in sample $(\mu g)$ : & 0.00 & 0.00 & 0.00 \\
\hline & Maximum corrected weight of impurity in sample $(\mu \mathrm{g})$ : & 0.27 & 0.21 & 0.48 \\
\hline & Measured concentration of impurity in sample $(\mu \mathrm{g} / \mathrm{ml})$ : & $<1.62 \mathrm{E}-03$ & $<1.62 \mathrm{E}-03$ & Co \\
\hline & Uncorrected weight of impurity in sample $(\mu g)$ : & $<0.23$ & $<0.18$ & $<0.41$ \\
\hline Co & Weight of impurity in blank $(\mu \mathrm{g})$ : & $<0.26$ & $<0.19$ & \\
\hline & Minimum corrected weight of impurity in sample $(\mu \mathrm{g})$ : & 0.00 & 0.00 & 0.00 \\
\hline & Maximum corrected weight of impurity in sample $(\mu \mathrm{g})$ : & 0.23 & 0.18 & 0.41 \\
\hline & Measured concentration of impurity in sample $(\mu \mathrm{g} / \mathrm{ml})$ : & $<8.00 \mathrm{E}-03$ & $<8.00 E-03$ & $\mathrm{Ni}$ \\
\hline & Uncorrected weight of impurity in sample $(\mu g)$ : & $<1.11$ & $<0.90$ & $<2.01$ \\
\hline $\mathrm{Ni}$ & Weight of impurity in blank $(\mu \mathrm{g})$ : & $<1.30$ & $<0.94$ & \\
\hline & Minimum corrected weight of impurity in sample $(\mu g)$ : & 0.00 & 0.00 & 0.00 \\
\hline & Maximum corrected weight of impurity in sample $(\mu \mathrm{g})$ : & 1.11 & 0.90 & 2.01 \\
\hline & Measured concentration of impurity in sample $(\mathrm{\mu g} / \mathrm{ml})$ : & $<1.00 \mathrm{E}-01$ & $2.09 E-01$ & Ca \\
\hline & Uncorrected weight of impurity in sample $(\mu \mathrm{g})$ : & $<13.90$ & 23.41 & $<37.31$ \\
\hline $\mathrm{Ca}$ & Weight of impurity in blank $(\mu \mathrm{gg})$ : & $<16.30$ & $<11.80$ & 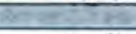 \\
\hline & Minimum corrected weight of impurity in sample $(\mu g)$ : & 0.00 & 11.61 & 11.61 \\
\hline & Maximum corrected weight of impurity in sample $(\mu g)$ : & 13.90 & 23.41 & 37.31 \\
\hline & Measured concentration of impurity in sample $(\mu \mathrm{g} / \mathrm{ml})$ : & 5.07E-01 & $1.88 \mathrm{E}-01$ & Al \\
\hline & Uncorrected weight of impurity in sample $(\mu \mathrm{g})$ : & 70.47 & 21.06 & 91.53 \\
\hline Al & Weight of impurity in blank $(\mu g)$ : & 11.70 & 3.95 & \\
\hline & Minimum corrected weight of impurity in sample $(\mu \mathrm{g})$ : & 58.77 & 17.10 & 75.87 \\
\hline & Maximum corrected weight of impurity in sample $(\mu g)$ : & 58.77 & 17.10 & 75.87 \\
\hline & Measured concentration of impurity in sample $(\mu \mathrm{g} / \mathrm{ml})$ : & $3.72 \mathrm{E}-02$ & $3.42 \mathrm{E}-02$ & Ti \\
\hline & Uncorrected weight of impurity in sample $(\mu \mathrm{g})$ : & 5.17 & 3.83 & 9.00 \\
\hline $\mathrm{Ti}$ & Weight of impurity in blank $(\mu g)$ : & $<1.30$ & $<0.94$ & \\
\hline & Minimum corrected weight of impurity in sample $(\mu \mathrm{g})$ : & 3.87 & 2.89 & 6.75 \\
\hline & Maximum corrected weight of impurity in sample $(\mu \mathrm{g})$ : & 5.17 & 3.83 & 9.00 \\
\hline & Measured concentration of impurity in sample $(\mu \mathrm{g} / \mathrm{ml})$ : & $2.58 \mathrm{E}-01$ & $7.90 \mathrm{E}-02$ & $\mathrm{v}$ \\
\hline & Uncorrected weight of impurity in sample $(\mu \mathrm{g})$ : & 35.86 & 8.85 & 44.71 \\
\hline v & Weight of impurity in blank $(\mu \mathrm{g})$ : & $<0.33$ & $<0.24$ & \\
\hline & Minimum corrected weight of impurity in sample $(\mu \mathrm{g})$ : & 35.54 & 8.61 & 44.15 \\
\hline & Maximum corrected weight of impurity in sample $(\mu \mathrm{g})$ : & 35.86 & 8.85 & 44.71 \\
\hline
\end{tabular}

Comments 
Data Report Form DRF-26A: Measurement of U Contamination or Impurities by Deconsolidation Leach

\begin{tabular}{|c|c|}
\hline 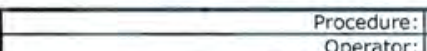 & AGR-CHAR-DAM-26 Rev. 1 \\
\hline $\begin{array}{r}\text { Operator: } \\
\text { Compact lot ID: }\end{array}$ & $\begin{array}{l}\text { Fred Montogemery } \\
\text { LEU07-OP1-Z }\end{array}$ \\
\hline Compact lot description: & AGR-2 UCO Baseline Fuel, from G73]-14-93072A \\
\hline Compact ID numbers: & $037,027,097,025,048$ \\
\hline DRF filename: & IImc-agr|AGRILeachBurn \\
\hline
\end{tabular}

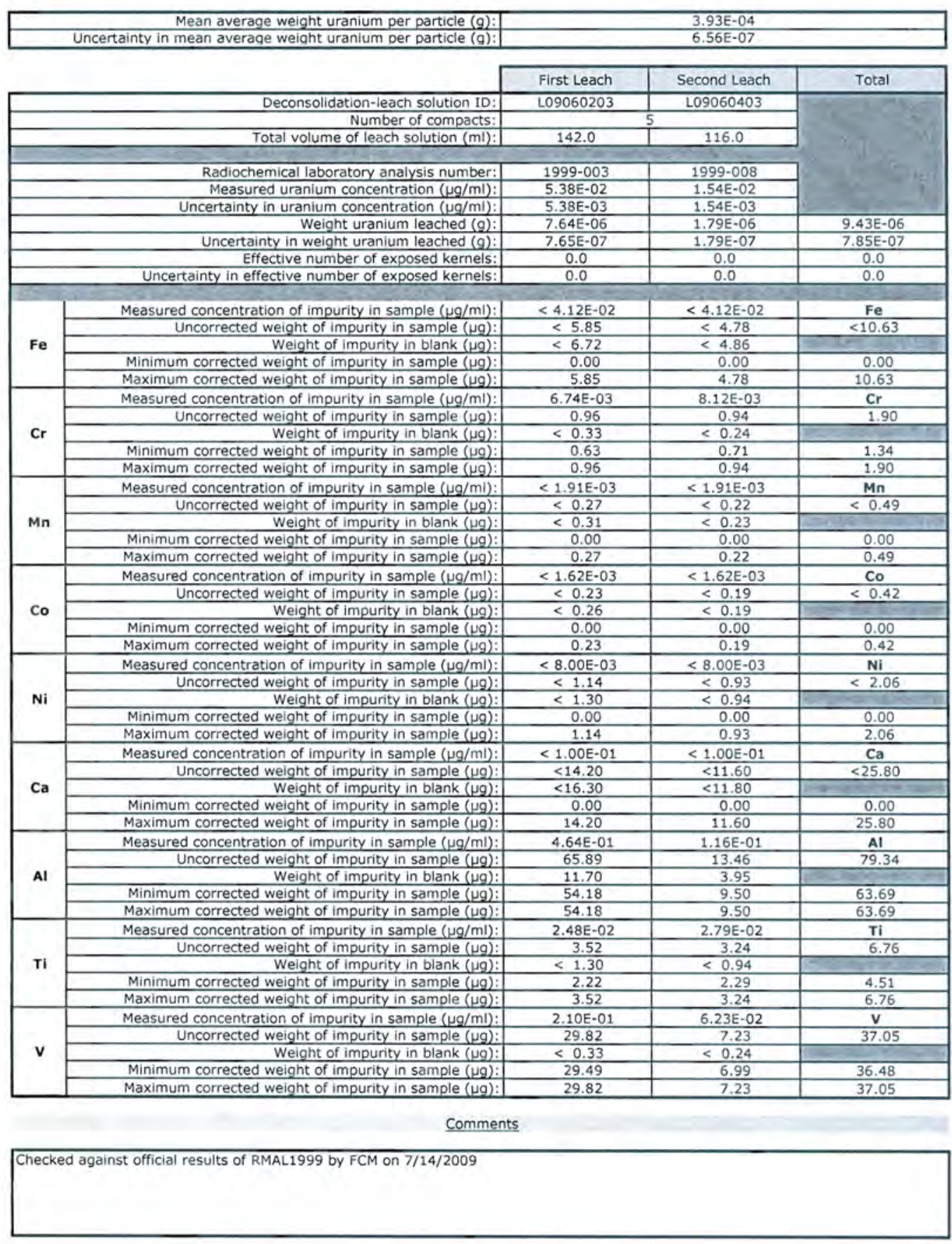

$7_{\text {ued C. Montgomery }}^{\text {Opertor }}-\frac{7.23 .09}{\text { Date }}$


Data Report Form DRF-26A: Measurement of U Contamination or Impurities by Deconsolidation Leach

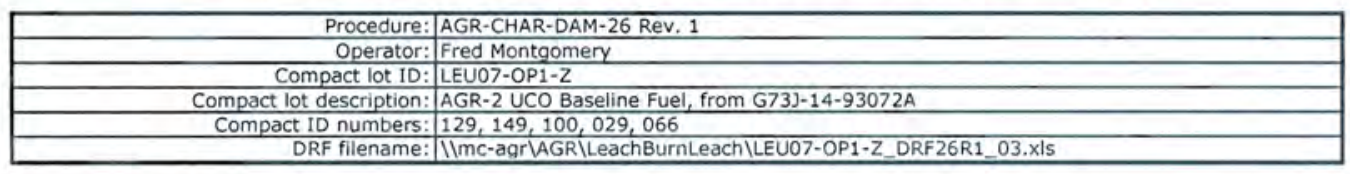

\begin{tabular}{|c|c|c|c|c|}
\hline \multirow{2}{*}{\multicolumn{2}{|c|}{$\begin{array}{r}\text { Mean average weight uranium per particle }(g) \text { : } \\
\text { Uncertainty in mean average weight uranium per particle }(q): \\
\end{array}$}} & \multicolumn{3}{|c|}{$\begin{array}{l}3.93 \mathrm{E}-04 \\
6.56 \mathrm{E}-07 \\
\end{array}$} \\
\hline & & First Leach & Second Leach & Total \\
\hline & Deconsolidation-leach solution ID: & L09060204 & L09060404 & \\
\hline & Number of compacts: & & & \\
\hline & Total volume of leach solution $(\mathrm{ml})$ : & 139.0 & 116.0 & \\
\hline & Dadinchemical tathoraton analucis number & & & \\
\hline & $\begin{array}{l}\text { Radiochemical laboratory analysis number: } \\
\text { Measured uranium concentration }(\mu \mathrm{g} / \mathrm{ml})\end{array}$ & $\frac{1999-004}{2.72 E+00}$ & $\frac{1999-009}{1.65 \mathrm{E}-01}$ & \\
\hline & Uncertainty in uranium concentration $(\mu \mathrm{g} / \mathrm{ml})$ : & $2.72 \mathrm{E}-01$ & $1.65 \mathrm{E}-02$ & \\
\hline & $\begin{array}{r}\text { Weight uranium leached }(g): \\
\end{array}$ & $3.78 \mathrm{E}-04$ & $1.91 \mathrm{E}-05$ & $3.97 E-04$ \\
\hline & Uncertainty in weight uranium leached $(g)$ : & $3.78 \mathrm{E}-05$ & $1.92 \mathrm{E}-06$ & $3.79 \mathrm{E}-05$ \\
\hline & Effective number of exposed kernels: & 1.0 & 0.0 & 1.0 \\
\hline & Uncertainty in effective number of exposed kernels: & 0.1 & 0.0 & 0.1 \\
\hline \multirow{5}{*}{$\mathrm{Fe}$} & Measured concentration of impurity in sample $(\mathrm{ug} / \mathrm{ml})$ : & $<4,12 \mathrm{E}-02$ & & \\
\hline & Uncorrected weight of impurity in sample $(\mu \mathrm{g})$ : & $<5.73$ & $<\frac{<.12 E-02}{<4.78}$ & $\frac{\mathrm{Fe}}{<10.51}$ \\
\hline & Weight of impurity in blank $(\mu g)$ : & $<6.72$ & $<4.86$ & \\
\hline & Minimum corrected weight of impurity in sample $(\mu g)$ : & 0.00 & 0.00 & 0.00 \\
\hline & Maximum corrected weight of impurity in sample $(\mu g)$ : & 5.73 & 4.78 & 10.51 \\
\hline \multirow{5}{*}{$\mathrm{Cr}$} & Measured concentration of impurity in sample $(\mathrm{\mu g} / \mathrm{ml})$ : & $1.23 \mathrm{E}-02$ & $6.59 \mathrm{E}-03$ & $\mathrm{Cr}$ \\
\hline & Uncorrected weight of impurity in sample $(\mu \mathrm{g})$ : & 1.71 & 0.76 & 2.47 \\
\hline & Weight of impurity in blank $(\mu g)$ : & $<0.33$ & $<0.24$ & \\
\hline & Minimum corrected weight of impurity in sample $(\mu g)$ : & 1.38 & 0.53 & 1.91 \\
\hline & Maximum corrected weight of impurity in sample $(\mu g)$ : & 1.71 & 0.76 & 2.47 \\
\hline \multirow{5}{*}{ Mn } & Measured concentration of impurity in sample $(\mu \mathrm{g} / \mathrm{ml})$ : & $<1.91 \mathrm{E}-03$ & $<1.91 \mathrm{E}-03$ & $\mathrm{Mn}$ \\
\hline & Uncorrected weight of impurity in sample $(\mu g)$ : & $<0.27$ & $<0.22$ & $<0.49$ \\
\hline & Weight of impurity in blank $(\mu g)$ : & $<0.31$ & $<0.23$ & \\
\hline & Minimum corrected weight of impurity in sample $(\mu g)$ : & 0.00 & 0.00 & 0.00 \\
\hline & Maximum corrected weight of impurity in sample $(\mu g)$ : & 0.27 & 0.22 & 0.49 \\
\hline \multirow{5}{*}{ co } & Measured concentration of impurity in sample $(\mathrm{\mu g} / \mathrm{ml})$ : & $<1.62 \mathrm{E}-03$ & $<1.62 \mathrm{E}-03$ & Co \\
\hline & Uncorrected weight of impurity in sample $(\mu \mathrm{g})$ : & $<0.23$ & $<0.19$ & $<0.41$ \\
\hline & Weight of impurity in blank $(\mu \mathrm{g})$ : & $<0.26$ & $<0.19$ & \\
\hline & Minimum corrected weight of impurity in sample $(\mu \mathrm{g})$ : & 0.00 & 0.00 & 0.00 \\
\hline & Maximum corrected weight of impurity in sample $(\mu g)$ : & 0.23 & 0.19 & 0.41 \\
\hline \multirow{5}{*}{$\mathrm{Ni}$} & Measured concentration of impurity in sample $(\mu \mathrm{g} / \mathrm{ml})$ : & $<8.00 \mathrm{E}-03$ & $<8.00 \mathrm{E}-03$ & $\mathrm{Ni}$ \\
\hline & Uncorrected weight of impurity in sample $(\mu \mathrm{g})$ : & $<1.11$ & $<0.93$ & $<2.04$ \\
\hline & Weight of impurity in blank $(\mu \mathrm{g})$ : & $<1.30$ & $<0.94$ & \\
\hline & Minimum corrected weight of impurity in sample $(\mu g)$ : & 0.00 & 0.00 & 0.00 \\
\hline & Maximum corrected weight of impurity in sample $(\mu \mathrm{g})$ : & 1.11 & 0.93 & 2.04 \\
\hline \multirow{5}{*}{$\mathrm{Ca}$} & Measured concentration of impurity in sample $(\mu \mathrm{g} / \mathrm{ml})$ : & $<1.00 \mathrm{E}-01$ & $<1.00 \mathrm{E}-01$ & $\mathrm{Ca}$ \\
\hline & Uncorrected weight of impurity in sample $(\mu g)$ : & $<13.90$ & $<11.60$ & $<25.50$ \\
\hline & Weight of impurity in blank $(\mu \mathrm{g})$ : & $<16.30$ & $<11.80$ & \\
\hline & Minimum corrected weight of impurity in sample $(\mu \mathrm{g})$ : & 0.00 & 0.00 & 0.00 \\
\hline & Maximum corrected weight of impurity in sample $(\mu \mathrm{g})$ : & 13.90 & 11.60 & 25.50 \\
\hline \multirow{5}{*}{ Al } & Measured concentration of impurity in sample $(\mu \mathrm{g} / \mathrm{ml})$ : & $5.08 \mathrm{E}-01$ & $1.44 E-01$ & Al \\
\hline & Uncorrected weight of impurity in sample $(\mu \mathrm{g})$ : & 70.61 & 16.70 & 87.32 \\
\hline & $\begin{array}{l}\text { Weight of impurity in blank }(\mu g) \text { : } \\
\text {. }\end{array}$ & 11.70 & 3.95 & \\
\hline & Minimum corrected weight of impurity in sample $(\mu g)$ : & 58.91 & 12.75 & 71.66 \\
\hline & Maximum corrected weight of impurity in sample ( $\mu \mathrm{g})$ : & 58.91 & 12.75 & 71.66 \\
\hline \multirow{5}{*}{$\mathbf{T i}$} & Measured concentration of impurity in sample $(\mu \mathrm{g} / \mathrm{ml})$ : & $4.47 E-02$ & $3.18 \mathrm{E}-02$ & $\mathrm{Ti}$ \\
\hline & Uncorrected weight of impurity in sample ( $\mu \mathrm{g})$ : & 6.21 & 3.69 & 9.90 \\
\hline & Weight of impurity in blank $(\mu \mathrm{g})$ : & $<1.30$ & $<0.94$ & 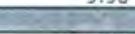 \\
\hline & Minimum corrected weight of impurity in sample $(\mu \mathrm{g})$ : & 4.91 & 2.74 & 7.65 \\
\hline & Maximum corrected weight of impurity in sample $(\mu g)$ : & 6.21 & 3.69 & 9.90 \\
\hline \multirow{5}{*}{$\mathbf{v}$} & Measured concentration of impurity in sample $(\mu \mathrm{g} / \mathrm{ml})$ : & $2.43 \mathrm{E}-01$ & $6.57 \mathrm{E}-02$ & $\mathrm{v}$ \\
\hline & Uncorrected weight of impurity in sample $(\mu g)$ : & 33.78 & 7.62 & 41.40 \\
\hline & Weight of impurity in blank $(\mu g)$ : & $<0.33$ & $<0.24$ & \\
\hline & Minimum corrected weight of impurity in sample $(\mu \mathrm{g})$ : & 33.45 & 7.39 & 40.84 \\
\hline & Maximum corrected weight of impurity in sample $(\mathrm{Hg})$ : & 33.78 & 7.62 & 41.40 \\
\hline
\end{tabular}

Comments

Checked against official results of RMAL1999 by FCM on 7/14/2009

Fud c. Montgomeuy 
Data Report Form DRF-26A: Measurement of U Contamination or Impurities by Deconsolidation Leach

\begin{tabular}{|c|c|}
\hline Procedure: & AGR-CHAR-DAM-26 Rev. 1 \\
\hline Operator: & Fred Montgomery \\
\hline Compact lot ID: & LEU07-OP1-Z \\
\hline Compact lot description: & AGR-2 UCO Baseline Fuel, from G73J-14-93072A \\
\hline Compact ID numbers: & Deconsolidation Leach Blank \\
\hline DRF filename: & IImc-agr\AGR\LeachBurnLeach|LEU07-OP1-Z_DRF26R1_03.xIs \\
\hline
\end{tabular}

\begin{tabular}{|c|c|c|c|c|}
\hline & Mean average weight uranium per particle $(\mathrm{g})$ : & \multirow{2}{*}{\multicolumn{3}{|c|}{$\begin{array}{l}3.93 \mathrm{E}-04 \\
6.56 \mathrm{E}-07\end{array}$}} \\
\hline & \multirow[t]{2}{*}{ Uncertainty in mean average weight uranium per particle (g): } & $6.56 \mathrm{E}-07$ & & \\
\hline & & First Leach & Second Leach & Total \\
\hline & Deconsolidation-leach solution ID: & L09060205 & L09060405 & \\
\hline & Number of compacts: & \multicolumn{2}{|c|}{ None } & \\
\hline \multirow{2}{*}{\multicolumn{4}{|c|}{ Total volume or leach solution (mi): }} & \\
\hline & & & & \\
\hline & Radiochemical laboratory analysis number: & $1999-005$ & $1999-010$ & \\
\hline & Measured uranium concentration $(\mu \mathrm{g} / \mathrm{ml})$ : & $2.36 \mathrm{E}-04$ & $<2.00 \mathrm{E}-04$ & \\
\hline & Uncertainty in uranium concentration $(\mu \mathrm{g} / \mathrm{ml})$ : & $2.36 \mathrm{E}-05$ & & \\
\hline & Weight uranium leached $(\mathrm{g})$ : & $3.85 \mathrm{E}-08$ & $<2.36 \mathrm{E}-08$ & $<6.21 \mathrm{E}-08$ \\
\hline & Uncertainty in weight uranium leached $(\mathrm{g})$ : & $3.85 \mathrm{E}-09$ & & \\
\hline & Effective number of exposed kernels: & 0.0 & 0.0 & 0.0 \\
\hline & Uncertainty in effective number of exposed kernels: & 0.0 & & \\
\hline & & & & \\
\hline \multirow{2}{*}{$\mathrm{Fe}$} & Measured concentration $(\mu \mathrm{g} / \mathrm{ml})$ : & $<4.12 \mathrm{E}-02$ & $<4.12 \mathrm{E}-02$ & $\mathrm{Fe}$ \\
\hline & Total weight of leached impurity $(\mu \mathrm{g})$ : & $<6.72$ & $<4.86$ & $<11.58$ \\
\hline \multirow{2}{*}{$\mathrm{Cr}$} & Measured concentration $(\mu \mathrm{g} / \mathrm{ml})$ : & $<2.00 \mathrm{E}-03$ & $<2.00 \mathrm{E}-03$ & $\mathrm{Cr}$ \\
\hline & Total weight of leached impurity $(\mu \mathrm{g})$ : & $<0.33$ & $<0.24$ & $<0.56$ \\
\hline \multirow{2}{*}{$\mathrm{Mn}$} & Measured concentration $(\mu \mathrm{g} / \mathrm{ml})$ : & $<1.91 \mathrm{E}-03$ & $<1.91 \mathrm{E}-03$ & $\mathrm{Mn}$ \\
\hline & Total weight of leached impurity $(\mu \mathrm{g})$ : & $<0.31$ & $<0.23$ & $<0.54$ \\
\hline \multirow{2}{*}{ Co } & Measured concentration $(\mu \mathrm{g} / \mathrm{ml})$ : & $<1.62 \mathrm{E}-03$ & $<1.62 \mathrm{E}-03$ & Co \\
\hline & Total weight of leached impurity $(\mu \mathrm{g})$ : & $<0.26$ & $<0.19$ & $<0.46$ \\
\hline \multirow{2}{*}{$\mathbf{N i}$} & Measured concentration $(\mu \mathrm{g} / \mathrm{ml})$ : & $<8.00 \mathrm{E}-03$ & $<8.00 \mathrm{E}-03$ & $\mathrm{Ni}$ \\
\hline & Total weight of leached impurity $(\mu \mathrm{g})$ : & $<1.30$ & $<0.94$ & $<2.25$ \\
\hline \multirow{2}{*}{$\mathrm{Ca}$} & Measured concentration $(\mu \mathrm{g} / \mathrm{ml})$ : & $<1.00 \mathrm{E}-01$ & $<1.00 \mathrm{E}-01$ & $\mathrm{Ca}$ \\
\hline & Total weight of leached impurity $(\mu \mathrm{g})$ : & $<16.30$ & $<11.80$ & $<28.10$ \\
\hline \multirow[b]{2}{*}{ Al } & Measured concentration $(\mu \mathrm{g} / \mathrm{ml})$ : & $7.18 \mathrm{E}-02$ & $3.35 \mathrm{E}-02$ & Al \\
\hline & Total weight of leached impurity $(\mu \mathrm{g})$ : & 11.70 & 3.95 & 15.66 \\
\hline \multirow{2}{*}{$\mathrm{Ti}$} & Measured concentration $(\mu \mathrm{g} / \mathrm{ml})$ : & $<8.00 \mathrm{E}-03$ & $<8.00 \mathrm{E}-03$ & $\mathrm{Ti}$ \\
\hline & Total weight of leached impurity $(\mu \mathrm{g})$ : & $<1.30$ & $<0.94$ & $<2.25$ \\
\hline \multirow{2}{*}{ v } & Measured concentration $(\mu \mathrm{g} / \mathrm{ml})$ : & $<2.00 \mathrm{E}-03$ & $<2.00 \mathrm{E}-03$ & $\mathrm{v}$ \\
\hline & Total weight of leached impurity $(\mu \mathrm{g})$ : & $<0.33$ & $<0.24$ & $<0.56$ \\
\hline
\end{tabular}

\section{Comments}

Checked against official results of RMAL1999 by FCM on 7/14/2009 
Data Report Form DRF-26B: Measurement of SiC Burn-Leach Defects or Impurities by Burn-Leach

\begin{tabular}{|r|l|}
\hline Procedure: & AGR-CHAR-DAM-26 Rev. 1 \\
\hline Operator: & Fred Montgomery \\
\hline Compact lot ID: & LEU07-OP1-Z \\
\hline Compact lot description: & AGR-2 UCO Baseline Fuel, from G73J-14-93072A \\
\hline Compact ID numbers: & $005,013,107,05,062$ \\
\hline DRF filename: & IImc-agr|AGRILeachBurnLeachILEU07-OP1-Z_DRF26R1_03.xIs \\
\hline
\end{tabular}

\begin{tabular}{|c|c|c|c|c|}
\hline \multicolumn{2}{|c|}{$\begin{array}{r}\text { Mean average weight uranium per particle }(g) \text { : } \\
\text { Uncertainty in mean average weight uranium per particle }(q): \\
\end{array}$} & \multicolumn{3}{|c|}{$\frac{3.93 E-04}{6.56 E-07}$} \\
\hline & & First Leach & Second Leach & Total \\
\hline & Burn-leach solution ID: & $B 09061601$ & $B 09061901$ & \\
\hline & Number of compacts: & & & \\
\hline & Total volume of leach solution $(\mathrm{ml}):$ & 49.0 & 51.5 & \\
\hline & Radiochemical laboratory analysis number: & $2014-001$ & $2014-006$ & \\
\hline & Measured uranium concentration $(\mathrm{\mu g} / \mathrm{ml}):$ & $\frac{2014001}{4.15 E-01}$ & $\frac{2014-000}{3.01 E-01}$ & \\
\hline & Uncertainty in uranium concentration $(\mu \mathrm{g} / \mathrm{ml})$ : & $4.15 \mathrm{E}-02$ & $3.01 \mathrm{E}-02$ & \\
\hline & Weight uranium leached $(g):$ & $2.03 E-05$ & $1.55 E-05$ & $3.58 \mathrm{E}-05$ \\
\hline & Uncertainty in weight uranium leached (g): & $2.05 E-06$ & $1.56 \mathrm{E}-06$ & $2.58 \mathrm{E}-06$ \\
\hline & Number of leached kernels: & 0.1 & 0.0 & 0.1 \\
\hline & Uncertainty in number of leached kernels: & 0.0 & 0.0 & 0.0 \\
\hline \multirow{5}{*}{$\mathrm{Fe}$} & & $7.24 E-02$ & & \\
\hline & Uncorrected weight of impurity in sample $(\mu g)$ : & 3.55 & $<2.12$ & $<5.67$ \\
\hline & Weight of impurity in blank $(\mu g)$ : & $<2.06$ & $<2.16$ & \\
\hline & Minimum corrected weight of impurity in sample $(\mu \mathrm{g})$ : & 1.49 & 0.00 & 1.49 \\
\hline & Maximum corrected weight of impurity in sample $(\mu \mathrm{g})$ : & 3.55 & 2.12 & 5.67 \\
\hline \multirow{5}{*}{$\mathrm{Cr}$} & Measured concentration of impurity in sample $(\mu \mathrm{g} / \mathrm{ml})$ : & $1.34 \mathrm{E}-02$ & $<2.00 \mathrm{E}-03$ & $\mathrm{Cr}$ \\
\hline & Uncorrected weight of impurity in sample $(\mu \mathrm{g})$ : & 0.66 & $<0.10$ & $<0.76$ \\
\hline & Weight of impurity in blank $(\mu g)$ : & $<0.10$ & $<0.11$ & \\
\hline & Minimum corrected weight of impurity in sample $(\mu \mathrm{g})$ : & 0.56 & 0.00 & 0.56 \\
\hline & Maximum corrected weight of impurity in sample $(\mu g)$ : & 0.66 & 0.10 & 0.76 \\
\hline \multirow{5}{*}{ Mn } & Measured concentration of impurity in sample $(\mu \mathrm{g} / \mathrm{ml})$ : & $<1.91 \mathrm{E}-03$ & $<1.91 \mathrm{E}-03$ & $\mathrm{Mn}$ \\
\hline & Uncorrected weight of impurity in sample $(\mu \mathrm{g})$ : & $<0.09$ & $<0.10$ & $<0.19$ \\
\hline & Weight of impurity in blank $(\mu g)$ : & $<0.10$ & $<0.10$ & \\
\hline & Minimum corrected weight of impurity in sample $(\mu \mathrm{g})$ : & 0.00 & 0.00 & 0.00 \\
\hline & Maximum corrected weight of impurity in sample $(u g)$ : & 0.09 & 0.10 & 0.19 \\
\hline \multirow{5}{*}{ co } & Measured concentration of impurity in sample $(\mu \mathrm{g} / \mathrm{ml})$ : & $<1.62 \mathrm{E}-03$ & $<1.62 \mathrm{E}-03$ & Co \\
\hline & Uncorrected weight of impurity in sample $(\mu g)$ : & $<0.08$ & $<0.08$ & $<0.16$ \\
\hline & Weight of impurity in blank $(\mu \mathrm{g})$ : & $<0.08$ & $<0.09$ & \\
\hline & Minimum corrected weight of impurity in sample $(\mu \mathrm{g})$ : & 0.00 & 0.00 & 0.00 \\
\hline & Maximum corrected weight of impurity in sample $(\mu g)$ : & 0.08 & 0.08 & 0.16 \\
\hline \multirow{5}{*}{$\mathrm{Ni}$} & Measured concentration of impurity in sample $(\mu \mathrm{g} / \mathrm{ml})$ : & $8.25 \mathrm{E}-03$ & $<8.00 \mathrm{E}-03$ & $\mathrm{Ni}$ \\
\hline & Uncorrected weight of impurity in sample $(\mu \mathrm{g})$ : & 0.40 & $<0.41$ & $<0.82$ \\
\hline & Weight of impurity in blank $(\mu \mathrm{g})$ : & $<0.40$ & $<0.42$ & \\
\hline & Minimum corrected weight of impurity in sample $(\mu g)$ : & 0.00 & 0.00 & 0.00 \\
\hline & Maximum corrected weight of impurity in sample $(\mu \mathrm{g})$ : & 0.40 & 0.41 & 0.82 \\
\hline \multirow{5}{*}{$\mathrm{Ca}$} & Measured concentration of impurity in sample $(\mathrm{\mu g} / \mathrm{ml})$ : & $1.65 E+00$ & $2.19 E+00$ & $\mathbf{C a}$ \\
\hline & Uncorrected weight of impurity in sample $(\mu q)$ : & 80.85 & 112.79 & 193.64 \\
\hline & Weight of impurity in blank $(\mu g)$ : & 7.15 & 49.77 & \\
\hline & Minimum corrected weight of impurity in sample $(\mu g)$ : & 73.70 & 63.02 & 136.72 \\
\hline & Maximum corrected weight of impurity in sample $(\mu g)$ : & 73.70 & 63.02 & 136.72 \\
\hline \multirow{5}{*}{ Al } & Measured concentration of impurity in sample $(\mu \mathrm{g} / \mathrm{ml})$ : & $1.30 E+00$ & $9.21 \mathrm{E}-02$ & Al \\
\hline & Uncorrected weight of impurity in sample $(\mu q)$ : & 63.70 & 4.74 & 68.44 \\
\hline & Weight of impurity in blank $(\mu \mathrm{g})$ : & 2.59 & 2.85 & 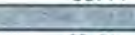 \\
\hline & Minimum corrected weight of impurity in sample $(\mu \mathrm{g})$ : & 61.11 & 1.90 & 63.01 \\
\hline & Maximum corrected weight of impurity in sample $(\mu \mathrm{g})$ : & 61.11 & 1.90 & 63.01 \\
\hline \multirow{5}{*}{$\mathbf{T i}$} & Measured concentration of impurity in sample $(\mu \mathrm{g} / \mathrm{ml})$ : & $1.43 E-01$ & $<8.00 \mathrm{E}-03$ & $\mathrm{Ti}$ \\
\hline & Uncorrected weight of impurity in sample $(\mu g)$ : & 7.01 & $<0.41$ & $<7.42$ \\
\hline & Weight of impurity in blank $(\mu \mathrm{g})$ : & $<0.40$ & $<0.42$ & \\
\hline & Minimum corrected weight of impurity in sample $(\mu \mathrm{g})$ : & 6.61 & 0.00 & 6.61 \\
\hline & Maximum corrected weight of impurity in sample $(\mu g)$ : & 7.01 & 0.41 & 7.42 \\
\hline \multirow{5}{*}{ v } & Measured concentration of impurity in sample $(\mu \mathrm{g} / \mathrm{ml})$ : & $9.85 \mathrm{E}-01$ & $1.35 \mathrm{E}-02$ & $\mathrm{v}$ \\
\hline & Uncorrected weight of impurity in sample $(\mu \mathrm{g})$ : & 48.27 & 0.70 & 48.96 \\
\hline & Weight of impurity in blank $(\mu \mathrm{g})$ : & $<0.10$ & $<0.11$ & 2 \\
\hline & Minimum corrected weight of impurity in sample $(\mu g)$ : & 48.17 & 0.59 & 48.76 \\
\hline & Maximum corrected weight of impurity in sample $(\mu g)$ : & 48.27 & 0.70 & 48.96 \\
\hline \multicolumn{5}{|c|}{ Comments } \\
\hline \multicolumn{5}{|c|}{ Checked against official results of RMAL2014 by FCM on 7/23/2009 } \\
\hline
\end{tabular}

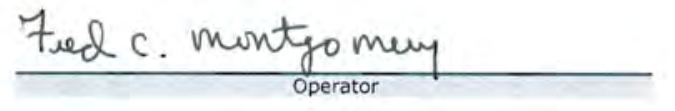

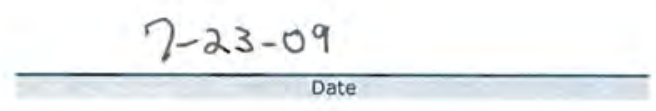


Data Report Form DRF-26B: Measurement of SiC Burn-Leach Defects or Impurities by Burn-Leach

\begin{tabular}{|r|r|}
\hline Procedure: & AGR-CHAR-DAM-26 Rev, 1 \\
\hline Operator: & Fred Montgomery \\
\hline Compact lot ID: & LEU07-OP1-Z \\
\hline Compact lot description: & AGR-2 UCO Baseline Fuel, from G73J-14-93072A \\
\hline Compact ID numbers: & $135,017,022,018,125$ \\
\hline DRF filename: & IImC-agr|AGRILeachBurnLeachILEU07-OP1-Z_DRF26R1_03.xIs \\
\hline
\end{tabular}

\begin{tabular}{|c|c|c|c|c|}
\hline \multirow{2}{*}{\multicolumn{2}{|c|}{$\begin{aligned} \text { Mean average weight uranium per particle }(g): \\
\text { Uncertainty in mean average weight uranium per particle }(\mathrm{g}): \\
\end{aligned}$}} & \multicolumn{3}{|c|}{$\begin{array}{l}3.93 \mathrm{E}-04 \\
6.56 \mathrm{E}-07 \\
\end{array}$} \\
\hline & & First Leach & Second Leach & Total \\
\hline & Burn-leach solution ID: & B09061602 & $B 09061902$ & \\
\hline & Number of compacts: & & & \\
\hline & Total volume of leach solution (ml): & 48.0 & 52.0 & \\
\hline & Radiochemical laboratory analysis number: & $2014-002$ & 2014-007 & \\
\hline & Measured uranium concentration $(\mu \mathrm{g} / \mathrm{ml})$ : & $1.14 \mathrm{E}+00$ & $4.47 E-02$ & \\
\hline & Uncertainty in uranium concentration $(\mathrm{\mu g} / \mathrm{ml})$ : & $1.14 \mathrm{E}-01$ & $4.47 \mathrm{E}-03$ & \\
\hline & Weight uranium leached $(\mathrm{g})$ : & $5.47 E-05$ & $2.32 E-06$ & $5.70 E-05$ \\
\hline & Uncertainty in weight uranium leached $(g)$ : & $5.52 \mathrm{E}-06$ & $2.34 E-07$ & $5.53 \mathrm{E}-06$ \\
\hline & Number of leached kernels: & 0.1 & 0.0 & 0.1 \\
\hline & Uncertainty in number of leached kernels: & 0.0 & 0.0 & 0.0 \\
\hline \multirow{5}{*}{$\mathrm{Fe}$} & Measured concentration of impurity in sample $(\mu \mathrm{g} / \mathrm{ml})$ : & $1.52 \mathrm{E}-01$ & $<4.12 \mathrm{E}-02$ & \\
\hline & Uncorrected weight of impurity in sample $(\mu \mathrm{gg})$ : & 7.30 & $<2.14$ & $<9.44$ \\
\hline & Weight of impurity in blank $(\mu \mathrm{g})$ : & $<2.06$ & $<2.16$ & \\
\hline & Minimum corrected weight of impurity in sample $(\mu \mathrm{g})$ : & 5.24 & 0.00 & 5.24 \\
\hline & Maximum corrected weight of impurity in sample $(\mu \mathrm{g})$ : & 7.30 & 2.14 & 9.44 \\
\hline \multirow{5}{*}{$\mathrm{Cr}$} & Measured concentration of impurity in sample $(\mu \mathrm{g} / \mathrm{ml})$ : & $1.63 \mathrm{E}-02$ & $<2.00 \mathrm{E}-03$ & $\mathrm{Cr}$ \\
\hline & Uncorrected weight of impurity in sample $(\mu g)$ : & 0.78 & $<0.10$ & $<0.89$ \\
\hline & Weight of impurity in blank $(\mathrm{\mu g})$ : & $<0.10$ & $<0.11$ & \\
\hline & Minimum corrected weight of impurity in sample $(\mu \mathrm{g})$ : & 0.68 & 0.00 & 0.68 \\
\hline & Maximum corrected weight of impurity in sample $(\mu g)$ : & 0.78 & 0.10 & 0.89 \\
\hline \multirow{5}{*}{ Mn } & Measured concentration of impurity in sample $(\mu \mathrm{g} / \mathrm{mi})$ : & $<1.91 \mathrm{E}-03$ & $<1.91 \mathrm{E}-03$ & $\mathrm{Mn}$ \\
\hline & Uncorrected weight of impurity in sample $(\mu g)$ : & $<0.09$ & $<0.10$ & $<0.19$ \\
\hline & Weight of impurity in blank $(\mu g)$ : & $<0.10$ & $<0.10$ & \\
\hline & Minimum corrected weight of impurity in sample $(\mu g)$ : & 0.00 & 0.00 & 0.00 \\
\hline & Maximum corrected weight of impurity in sample $(\mu \mathrm{g})$ : & 0.09 & 0.10 & 0.19 \\
\hline \multirow{5}{*}{ co } & Measured concentration of impurity in sample $(\mu \mathrm{g} / \mathrm{ml})$ : & $<1.62 \mathrm{E}-03$ & $<1.62 \mathrm{E}-03$ & Co \\
\hline & Uncorrected weight of impurity in sample $(\mu g)$ : & $<0.08$ & $<0.08$ & $<0.16$ \\
\hline & Weight of impurity in blank $(\mu g)$ : & $<0.08$ & $<0.09$ & \\
\hline & Minimum corrected weight of impurity in sample $(\mu g)$ : & 0.00 & 0.00 & 0.00 \\
\hline & Maximum corrected weight of impurity in sample $(\mu g)$ : & 0.08 & 0.08 & 0.16 \\
\hline \multirow{5}{*}{$\mathrm{Ni}$} & Measured concentration of impurity in sample $(\mu \mathrm{g} / \mathrm{ml})$ : & 1.07E-02 & $<8.00 \mathrm{E}-03$ & $\mathrm{Ni}$ \\
\hline & Uncorrected weight of impurity in sample $(\mu \mathrm{g})$ : & 0.51 & $<0.42$ & $<0.93$ \\
\hline & Weight of impurity in blank $(\mu \mathrm{g})$ : & $<0.40$ & $<0.42$ & \\
\hline & Minimum corrected weight of impurity in sample $(\mu g)$ : & 0.11 & 0.00 & 0.11 \\
\hline & Maximum corrected weight of impurity in sample $(\mu q)$ : & 0.51 & 0.42 & 0.93 \\
\hline \multirow{5}{*}{ Ca } & Measured concentration of impurity in sample $(\mu \mathrm{g} / \mathrm{mll})$ : & $1.92 E+00$ & $2.40 E+00$ & $\mathrm{Ca}$ \\
\hline & Uncorrected weight of impurity in sample $(\mu \mathrm{g})$ : & 92.16 & 124.80 & 216.96 \\
\hline & Weight of impurity in blank $(\mu g)$ : & 7.15 & 49.77 & \\
\hline & Minimum corrected weight of impurity in sample $(\mu g)$ : & 85.01 & 75.03 & 160.04 \\
\hline & Maximum corrected weight of impurity in sample $(\mu \mathrm{g})$ : & 85.01 & 75.03 & 160.04 \\
\hline \multirow{5}{*}{ Al } & Measured concentration of impurity in sample $(\mu \mathrm{g} / \mathrm{ml})$ : & $1.35 E+00$ & $1.31 \mathrm{E}-01$ & Al \\
\hline & Uncorrected weight of impurity in sample $(\mu g)$ : & 64.80 & 6.81 & 71.61 \\
\hline & $\begin{array}{l}\text { Weight of impurity in blank }(\mu \mathrm{g}) \text { : } \\
\end{array}$ & 2.59 & 2.85 & \\
\hline & Minimum corrected weight of impurity in sample $(\mu g)$ : & 62.21 & 3.97 & 66.18 \\
\hline & Maximum corrected weight of impurity in sample $(\mu g)$ : & 62.21 & 3.97 & 66.18 \\
\hline \multirow{5}{*}{$\mathrm{Ti}$} & Measured concentration of impurity in sample $(\mu \mathrm{g} / \mathrm{ml})$ : & $1.10 \mathrm{E}-01$ & $<8.00 \mathrm{E}-03$ & $\mathrm{Ti}$ \\
\hline & Uncorrected weight of impurity in sample $(\mu q)$ : & 5.28 & $<0.42$ & $<5.70$ \\
\hline & Weight of impurity in blank $(\mu g)$ : & $<0.40$ & $<0.42$ & 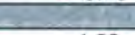 \\
\hline & Minimum corrected weight of impurity in sample $(\mu q)$ : & 4.88 & 0.00 & 4.88 \\
\hline & Maximum corrected weight of impurity in sample $(\mu g)$ : & 5.28 & 0.42 & 5.70 \\
\hline \multirow{5}{*}{ v } & Measured concentration of impurity in sample $(\mu \mathrm{g} / \mathrm{ml})$ : & $1.03 E+00$ & $1.28 \mathrm{E}-02$ & v \\
\hline & Uncorrected weight of impurity in sample $(\mu \mathrm{g})$ : & 49.44 & 0.67 & 50.11 \\
\hline & Weight of impurity in blank $(\mu \mathrm{g})$ : & $<0.10$ & $<0.11$ & \\
\hline & Minimum corrected weight of impurity in sample $(\mu g)$ : & 49.34 & 0.56 & 49.90 \\
\hline & Maximum corrected weight of impurity in sample $(\mu g)$ : & 49.44 & 0.67 & 50.11 \\
\hline
\end{tabular}

Comments

Fied C. Montyomuy

$7-23-09$ 
Data Report Form DRF-268: Measurement of SIC Burn-Leach Defects or Impurities by Burn-Leach

\begin{tabular}{|c|c|}
\hline Procedure: & AGR-CHAR-DAM-26 \\
\hline Operator: & Fred Montgomery \\
\hline Compact lot ID: & LEU07-OP1-Z \\
\hline Compact lot description: & AGR-2 UCO Baseline Fuel, from G73J-14-93072A \\
\hline Compact ID numbers: & $037,027,097,025,048$ \\
\hline DRF filename: & |Imc-agr|AGR|LeachBurnLeach|LEU07-OP1-Z_DRF26R1_03.x|s \\
\hline
\end{tabular}

\begin{tabular}{|c|c|c|c|c|}
\hline & $\begin{array}{l}\text { Mean average weight uranium per particle }(g) \text { : } \\
\text { Uncertainty in mean average weight uranium per particle }(q) \text { : }\end{array}$ & & $\begin{array}{l}3.93 E-04 \\
6.56 E-07\end{array}$ & \\
\hline & & First Leach & Second Leach & Total \\
\hline & Burn-leach solution ID: & B09061603 & $B 09061903$ & \\
\hline & Number of compacts: & & & \\
\hline & Total volume of leach solution $(\mathrm{ml})$ : & 50.5 & 52.0 & \\
\hline & & & & \\
\hline & $\begin{array}{l}\text { Radiochemical laboratory analysis number: } \\
\text { Measured uranium concentration }(\mathrm{\mu g} / \mathrm{ml}):\end{array}$ & $\frac{2014-003}{2.88 E-01}$ & $\frac{2014-008}{1.09 \mathrm{E}-02}$ & \\
\hline & $\begin{array}{l}\text { Measured uranium concentration }(\mu \mathrm{g} / \mathrm{ml}) \text { : } \\
\text { Uncertainty in uranium concentration }(\mu \mathrm{g} / \mathrm{ml}) \text { : }\end{array}$ & $2.88 \mathrm{E}-02$ & $1.09 \mathrm{E}-03$ & \\
\hline & Weight uranium leached $(g):$ & $1.45 \mathrm{E}-05$ & $5.67 E-07$ & $1.51 \mathrm{E}-05$ \\
\hline & Uncertainty in weight uranium leached $(g)$ : & $1.47 \mathrm{E}-06$ & $5.71 E-08$ & $1.47 \mathrm{E}-06$ \\
\hline & Number of leached kernels: & 0.0 & 0.0 & 0.0 \\
\hline & Uncertainty in number of leached kernels: & 0.0 & 0.0 & 0.0 \\
\hline & Measured concentration of impurity in sample $(u \mathrm{~g} / \mathrm{ml})$ : & $8.88 \mathrm{E}-02$ & $<4,12 \mathrm{E}-02$ & $\mathrm{Fe}$ \\
\hline & Uncorrected weight of impurity in sample $(\mu g)$ : & 4.48 & $<2.14$ & $<6.63$ \\
\hline $\mathrm{Fe}$ & Weight of impurity in blank $(\mu g)$ : & $<2.06$ & $<2.16$ & \\
\hline & Minimum corrected weight of impurity in sample $(\mu g)$ : & 2.42 & 0.00 & 2.42 \\
\hline & Maximum corrected weight of impurity in sample $(\mu g)$ : & 4.48 & 2.14 & 6.63 \\
\hline & Measured concentration of impurity in sample $(\mu \mathrm{g} / \mathrm{ml})$ : & $1.56 \mathrm{E}-02$ & $<2.00 \mathrm{E}-03$ & $\mathrm{Cr}$ \\
\hline & Uncorrected weight of impurity in sample $(\mu g)$ : & 0.79 & $<0.10$ & $<0.89$ \\
\hline $\mathrm{Cr}$ & Weight of impurity in blank $(\mu g)$ : & $<0.10$ & $<0.11$ & \\
\hline & Minimum corrected weight of impurity in sample $(\mu g)$ : & 0.69 & 0.00 & 0.69 \\
\hline & Maximum corrected weight of impurity in sample $(\mu \mathrm{g})$ : & 0.79 & 0.10 & 0.89 \\
\hline & Measured concentration of impurity in sample $(\mu \mathrm{g} / \mathrm{ml})$ : & $<1.91 \mathrm{E}-03$ & $<1.91 \mathrm{E}-03$ & $\mathrm{Mn}$ \\
\hline & Uncorrected weight of impurity in sample $(\mu \mathrm{g})$ : & $<0.10$ & $<0.10$ & $<0.20$ \\
\hline Mn & Weight of impurity in blank $(\mu g)$ : & $<0.10$ & $<0.10$ & \\
\hline & Minimum corrected weight of impurity in sample $(\mu g)$ : & 0.00 & 0.00 & 0.00 \\
\hline & Maximum corrected weight of impurity in sample $(\mu g)$ : & 0.10 & 0.10 & 0.20 \\
\hline & Measured concentration of impurity in sample $(\mu \mathrm{g} / \mathrm{ml})$ : & $<1.62 \mathrm{E}-03$ & $<1.62 \mathrm{E}-03$ & Co \\
\hline & Uncorrected weight of impurity in sample $(\mu q)$ : & $<0.08$ & $<0.08$ & $<0.17$ \\
\hline co & Weight of impurity in blank $(\mu \mathrm{g})$ : & $<0.08$ & $<0.09$ & 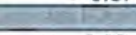 \\
\hline & Minimum corrected weight of impurity in sample $(\mu \mathrm{g})$ : & 0.00 & 0.00 & 0.00 \\
\hline & Maximum corrected weight of impurity in sample $(\mu q)$ : & 0.08 & 0.08 & 0.17 \\
\hline & Measured concentration of impurity in sample $(\mu \mathrm{g} / \mathrm{ml})$ : & $9.30 \mathrm{E}-03$ & $<8.00 \mathrm{E}-03$ & $\mathrm{Ni}$ \\
\hline & Uncorrected weight of impurity in sample $(\mu \mathrm{g})$ : & 0.47 & $<0.42$ & $<0.89$ \\
\hline $\mathrm{Ni}$ & Weight of impurity in blank $(\mu \mathrm{g})$ : & $<0.40$ & $<0.42$ & \\
\hline & Minimum corrected weight of impurity in sample $(\mu \mathrm{g})$ : & 0.07 & 0.00 & 0.07 \\
\hline & Maximum corrected weight of impurity in sample $(\mu g)$ : & 0.47 & 0.42 & 0.89 \\
\hline & Measured concentration of impurity in sample $(\mu \mathrm{g} / \mathrm{ml})$ : & $2.13 \mathrm{E}+00$ & $2.25 E+00$ & $\mathrm{Ca}$ \\
\hline & Uncorrected weight of impurity in sample $(\mu \mathrm{g})$ : & 107.57 & 117.00 & 224.57 \\
\hline $\mathrm{Ca}$ & Weight of impurity in blank $(\mu \mathrm{g})$ : & 7.15 & 49.77 & \\
\hline & Minimum corrected weight of impurity in sample $(\mathrm{\mu g})$ : & 100.42 & 67.23 & 167.65 \\
\hline & Maximum corrected weight of impurity in sample $(\mathrm{\mu g})$ : & 100.42 & 67.23 & 167.65 \\
\hline & Measured concentration of impurity in sample $(\mu \mathrm{g} / \mathrm{ml})$ : & $1.26 \mathrm{E}+00$ & $1.49 \mathrm{E}-01$ & Al \\
\hline & Uncorrected weight of impurity in sample $(\mu \mathrm{g})$ : & 63.63 & 7.75 & 71.38 \\
\hline Al & $\begin{array}{l}\text { Weight of impurity in blank }(\mu g) \text { : } \\
\text {. }\end{array}$ & 2.59 & 2.85 & 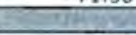 \\
\hline & Minimum corrected weight of impurity in sample $(\mu \mathrm{g})$ : & 61.04 & 4.90 & 65.94 \\
\hline & Maximum corrected weight of impurity in sample $(\mu g)$ : & 61.04 & 4.90 & 65.94 \\
\hline & Measured concentration of impurity in sample $(\mathrm{\mu g} / \mathrm{ml})$ : & $1.52 \mathrm{E}-01$ & $8.53 \mathrm{E}-03$ & $\mathrm{Ti}$ \\
\hline & Uncorrected weight of impurity in sample $(\mu \mathrm{g})$ : & 7.68 & 0.44 & 8.12 \\
\hline $\mathbf{T i}$ & Weight of impurity in blank $(\mu g)$ : & $<0.40$ & $<0.42$ & \\
\hline & Minimum corrected weight of impurity in sample $(\mu \mathrm{g})$ : & 7.28 & 0.02 & 7.30 \\
\hline & Maximum corrected weight of impurity in sample $(\mu \mathrm{g})$ : & 7.68 & 0.44 & 8.12 \\
\hline & Measured concentration of impurity in sample $(\mu \mathrm{g} / \mathrm{ml})$ : & $9.01 \mathrm{E}-01$ & $1.33 \mathrm{E}-02$ & $\mathrm{v}$ \\
\hline & Uncorrected weight of impurity in sample $(\mu \mathrm{g})$ : & 45.50 & 0.69 & 46.19 \\
\hline v & Weight of impurity in blank $(\mathrm{\mu g})$ : & $<0.10$ & $<0.11$ & \\
\hline & Minimum corrected weight of impurity in sample $(\mu \mathrm{g})$ : & 45.40 & 0.59 & 45.99 \\
\hline & Maximum corrected weight of impurity in sample $(\mu \mathrm{g})$ : & 45.50 & 0.69 & 46.19 \\
\hline & Commer & & & \\
\hline Che & against official results of RMAL2014 by FCM on 7/23/2009 & & & \\
\hline
\end{tabular}

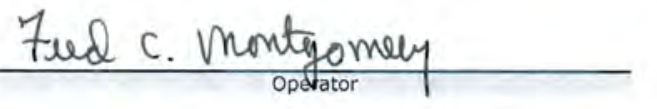

$7-23-09$ 
Data Report Form DRF-26B: Measurement of SiC Burn-Leach Defects or Impurities by Burn-Leach

\begin{tabular}{|r|r|}
\hline Procedure: & AGR-CHAR-DAM-26 Rev. 1 \\
\hline Operator: & Fred Montgomery \\
\hline Compact lot ID: & LEU07-OP1-Z \\
\hline Compact lot description: & AGR-2 UCO Baseline Fuel, from G73J-14-93072A \\
\hline Compact ID numbers: & $129,149,100,029,066$ \\
\hline DRF filename: & IImC-agr(LGRILeachBurnLeachILEU07-OP1-Z_DRF26R1_03.xIs \\
\hline
\end{tabular}

\begin{tabular}{|c|c|c|c|c|}
\hline & $\begin{array}{l}\text { Mean average weight uranium per particle }(g) \text { : } \\
\text { Uncertainty in mean average weight uranium per particle }(\mathrm{g}) \text { : }\end{array}$ & & $\begin{array}{l}3.93 \mathrm{E}-04 \\
6.56 \mathrm{E}-07\end{array}$ & \\
\hline & & First Leach & Second Leach & Total \\
\hline & Burn-leach solution ID: & B09061604 & 809061904 & \\
\hline & Number of compacts: & & & \\
\hline & Total volume of leach solution $(\mathrm{ml})$ : & 49.5 & 51.5 & \\
\hline & Radiochemical laboratory analysis number: & $2014-004$ & $2014-009$ & \\
\hline & Measured uranium concentration $(\mathrm{\mu g} / \mathrm{ml})$ : & $3.95 \mathrm{E}-01$ & $7.78 \mathrm{E}-03$ & \\
\hline & Uncertainty in uranium concentration $(\mu \mathrm{g} / \mathrm{ml})$ : & $3.95 \mathrm{E}-02$ & $7.78 \mathrm{E}-04$ & \\
\hline & Weight uranium leached (g): & $1.96 \mathrm{E}-05$ & $4.01 \mathrm{E}-07$ & $2.00 \mathrm{E}-05$ \\
\hline & Uncertainty in weight uranium leached $(\mathrm{g})$ : & $1.97 \mathrm{E}-06$ & $4.04 E-08$ & $1.97 \mathrm{E}-06$ \\
\hline & Number of leached kernels: & 0.0 & 0.0 & 0.1 \\
\hline & Uncertainty in number of leached kernels: & 0.0 & 0.0 & 0.0 \\
\hline & Measured concentration of impurity in sample $(\mathrm{\mu g} / \mathrm{ml})$ : & $1.13 \mathrm{E}-01$ & $5.22 \mathrm{E}-02$ & $\mathrm{Fe}$ \\
\hline & Uncorrected weight of impurity in sample $(\mu g)$ : & 5.59 & 2.69 & 8.28 \\
\hline $\mathbf{F e}$ & Weight of impurity in blank $(\mu g)$ : & $<2.06$ & $<2.16$ & \\
\hline & Minimum corrected weight of impurity in sample $(\mu \mathrm{g})$ : & 3.53 & 0.53 & 4.06 \\
\hline & Maximum corrected weight of impurity in sample $(\mu g)$ : & 5.59 & 2.69 & 8.28 \\
\hline & Measured concentration of impurity in sample $(\mathrm{\mu g} / \mathrm{ml})$ : & $1.62 \mathrm{E}-02$ & $<2.00 \mathrm{E}-03$ & $\mathrm{Cr}$ \\
\hline & Uncorrected weight of impurity in sample $(\mu g)$ : & 0.80 & $<0.10$ & $<0.90$ \\
\hline $\mathrm{Cr}$ & Weight of impurity in blank $(\mu g)$ : & $<0.10$ & $<0.11$ & \\
\hline & Minimum corrected weight of impurity in sample $(\mu g)$ : & 0.70 & 0.00 & 0.70 \\
\hline & Maximum corrected weight of impurity in sample $(\mu g)$ : & 0.80 & 0.10 & 0.90 \\
\hline & Measured concentration of impurity in sample $(\mathrm{\mu g} / \mathrm{ml})$ : & $<1.91 \mathrm{E}-03$ & $<1.91 \mathrm{E}-03$ & $\mathrm{Mn}$ \\
\hline & Uncorrected weight of impurity in sample $(\mu g)$ : & $<0.09$ & $<0.10$ & $<0.19$ \\
\hline Mn & Weight of impurity in blank $(\mu \mathrm{g})$ : & $<0.10$ & $<0.10$ & \\
\hline & Minimum corrected weight of impurity in sample $(\mu g)$ : & 0.00 & 0.00 & 0.00 \\
\hline & Maximum corrected weight of impurity in sample $(\mu g)$ : & 0.09 & 0.10 & 0.19 \\
\hline & Measured concentration of impurity in sample $(\mathrm{\mu g} / \mathrm{ml})$ : & $<1.62 \mathrm{E}-03$ & $<1.62 \mathrm{E}-03$ & Co \\
\hline & Uncorrected weight of impurity in sample $(\mu g)$ : & $<0.08$ & $<0.08$ & $<0.16$ \\
\hline co & Weight of impurity in blank $(\mu g)$ : & $<0.08$ & $<0.09$ & \\
\hline & Minimum corrected weight of impurity in sample $(\mathrm{\mu g})$ : & 0.00 & 0.00 & 0.00 \\
\hline & Maximum corrected weight of impurity in sample $(\mu g)$ : & 0.08 & 0.08 & 0.16 \\
\hline & Measured concentration of impurity in sample $(\mathrm{\mu g} / \mathrm{ml})$ : & $1.06 \mathrm{E}-02$ & $<8.00 E-03$ & $\mathrm{Ni}$ \\
\hline & Uncorrected weight of impurity in sample $(\mu g)$ : & 0.52 & $<0.41$ & $<0.94$ \\
\hline $\mathrm{Ni}$ & Weight of impurity in blank $(\mu \mathrm{g})$ : & $<0.40$ & $<0.42$ & जी \\
\hline & Minimum corrected weight of impurity in sample $(\mu g)$ : & 0.12 & 0.00 & 0.12 \\
\hline & Maximum corrected weight of impurity in sample $(\mu q)$ : & 0.52 & 0.41 & 0.94 \\
\hline & Measured concentration of impurity in sample $(\mu \mathrm{g} / \mathrm{ml})$ : & $2.03 E+00$ & $3.02 E+00$ & $\mathrm{Ca}$ \\
\hline & Uncorrected weight of impurity in sample $(\mu \mathrm{g})$ : & 100.49 & 155.53 & 256.02 \\
\hline $\mathrm{Ca}$ & Weight of impurity in blank $(\mu g)$ : & 7.15 & 49.77 & \\
\hline & Minimum corrected weight of impurity in sample $(\mu q)$ : & 93.34 & 105.76 & 199.10 \\
\hline & Maximum corrected weight of impurity in sample $(\mu \mathrm{g})$ : & 93.34 & 105.76 & 199.10 \\
\hline & Measured concentration of impurity in sample $(\mu \mathrm{g} / \mathrm{ml})$ : & $1.30 E+00$ & $1.61 \mathrm{E} \cdot 01$ & Al \\
\hline & Uncorrected weight of impurity in sample $(\mu \mathrm{g})$ : & 64.35 & 8.29 & 72.64 \\
\hline Al & Weight of impurity in blank $(\mu g)$ : & 2.59 & 2.85 & \\
\hline & Minimum corrected weight of impurity in sample $(\mu g)$ : & 61.76 & 5.45 & 67.21 \\
\hline & Maximum corrected weight of impurity in sample $(\mu q)$ : & 61.76 & 5.45 & 67.21 \\
\hline & Measured concentration of impurity in sample $(\mu \mathrm{g} / \mathrm{ml})$ : & $1.09 \mathrm{E}-01$ & $8.24 \mathrm{E}-03$ & $\mathrm{Ti}$ \\
\hline & Uncorrected weight of impurity in sample $(\mu g)$ : & 5.40 & 0.42 & 5.82 \\
\hline $\mathbf{T i}$ & Weight of impurity in blank $(\mu \mathrm{g})$ : & $<0.40$ & $<0.42$ & \\
\hline & Minimum corrected weight of impurity in sample $(\mu \mathrm{g})$ : & 5.00 & 0.00 & 5.00 \\
\hline & Maximum corrected weight of impurity in sample $(\mu \mathrm{g})$ : & 5.40 & 0.42 & 5.82 \\
\hline & Measured concentration of impurity in sample $(\mu \mathrm{g} / \mathrm{ml})$ : & $9.19 \mathrm{E}-01$ & $9.93 \mathrm{E}-03$ & $\mathrm{v}$ \\
\hline & Uncorrected weight of impurity in sample $(\mu g)$ : & 45.49 & 0.51 & 46.00 \\
\hline $\mathbf{v}$ & Weight of impurity in blank $(\mu g)$ : & $<0.10$ & $<0.11$ & \\
\hline & Minimum corrected weight of impurity in sample $(\mu g)$ : & 45.39 & 0.41 & 45.80 \\
\hline & Maximum corrected weight of impurity in sample $(\mu g)$ : & 45.49 & 0.51 & 46.00 \\
\hline
\end{tabular}

Comments 
Data Report Form DRF-26B: Measurement of SiC Burn-Leach Defects or Impurities by Burn-Leach

\begin{tabular}{|c|c|}
\hline Procedure: & AGR-CHAR-DAM-26 Rev. 1 \\
\hline Operator: & Fred Montgomery \\
\hline Compact lot ID: & LEU07-OP1-Z \\
\hline Compact lot description: & AGR-2 UCO Baseline Fuel, from G73]-14-93072A \\
\hline Compact ID numbers: & Burn-Leach Blank \\
\hline DRF filename: & IImc-agr\AGR\LeachBurnLeach\LEU07-OP1-Z_DRF26R1_03.xIs \\
\hline
\end{tabular}

\begin{tabular}{|c|c|c|c|c|}
\hline & Mean average weight uranium per particle $(g)$ : & \multirow{2}{*}{\multicolumn{3}{|c|}{$\begin{array}{l}3.93 \mathrm{E}-04 \\
6.56 \mathrm{E}-07\end{array}$}} \\
\hline & \multirow[t]{2}{*}{ Uncertainty in mean average weight uranium per particle $(g)$ : } & & & \\
\hline & & First Leach & Second Leach & Total \\
\hline & Burn-leach solution ID: & B09061605 & B09061905 & \\
\hline \multirow{2}{*}{\multicolumn{2}{|c|}{$\begin{array}{r}\text { Number of compacts: } \\
\text { Total volume of leach solution }(\mathrm{ml}):\end{array}$}} & \multicolumn{2}{|c|}{ None } & \\
\hline & & \multirow{2}{*}{\multicolumn{2}{|c|}{\begin{tabular}{l|l} 
& 52.5 \\
\end{tabular}}} & \\
\hline \multirow{2}{*}{\multicolumn{2}{|c|}{ Radiochemical laboratory analysis number: }} & & & \\
\hline & & $2014-005$ & $2014-010$ & \\
\hline & Measured uranium concentration $(\mu \mathrm{g} / \mathrm{ml})$ : & $<5.00 \mathrm{E}-04$ & $<5.00 \mathrm{E}-04$ & \\
\hline \multicolumn{2}{|r|}{ Uncertainty in uranium concentration $(\mu \mathrm{g} / \mathrm{ml})$ : } & & & \\
\hline & Weight uranium leached $(g)$ : & $<2.50 \mathrm{E}-08$ & $<2.63 \mathrm{E}-08$ & $<5.13 \mathrm{E}-08$ \\
\hline \multicolumn{2}{|r|}{ Uncertainty in weight uranium leached $(g)$ : } & & & \\
\hline & Number of leached kernels: & 0.0 & 0.0 & 0.0 \\
\hline \multicolumn{2}{|r|}{ Uncertainty in number of leached kernels: } & & & \\
\hline \multirow{3}{*}{$\mathrm{Fe}$} & 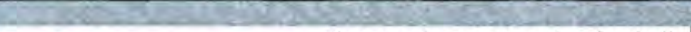 & & & \\
\hline & Measured concentration $(\mu \mathrm{g} / \mathrm{ml})$ : & $<4.12 \mathrm{E}-02$ & $<4.12 \mathrm{E}-02$ & $\mathrm{Fe}$ \\
\hline & Total weight of leached impurity $(\mu g)$ : & $<2.06$ & $<2.16$ & $<4.22$ \\
\hline \multirow{2}{*}{$\mathrm{Cr}$} & Measured concentration $(\mu \mathrm{g} / \mathrm{ml})$ : & $<2.00 \mathrm{E}-03$ & $<2.00 \mathrm{E}-03$ & $\mathrm{Cr}$ \\
\hline & Total weight of leached impurity $(\mu \mathrm{g})$ : & $<0.10$ & $<0.11$ & $<0.21$ \\
\hline \multirow{2}{*}{ Mn } & Measured concentration $(\mu \mathrm{g} / \mathrm{ml})$ : & $<1.91 \mathrm{E}-03$ & $<1.91 \mathrm{E}-03$ & Mn \\
\hline & Total weight of leached impurity $(\mu \mathrm{g})$ : & $<0.10$ & $<0.10$ & $<0.20$ \\
\hline \multirow{2}{*}{ Co } & Measured concentration $(\mu \mathrm{g} / \mathrm{ml})$ : & $<1.62 \mathrm{E}-03$ & $<1.62 \mathrm{E}-03$ & Co \\
\hline & Total weight of leached impurity $(\mu \mathrm{g})$ : & $<0.08$ & $<0.09$ & $<0.17$ \\
\hline \multirow{2}{*}{$\mathrm{Ni}$} & Measured concentration $(\mu \mathrm{g} / \mathrm{ml})$ : & $<8.00 \mathrm{E}-03$ & $<8.00 \mathrm{E}-03$ & $\mathrm{Ni}$ \\
\hline & Total weight of leached impurity $(\mu g)$ : & $<0.40$ & $<0.42$ & $<0.82$ \\
\hline \multirow{2}{*}{$\mathrm{Ca}$} & Measured concentration $(\mu \mathrm{g} / \mathrm{ml})$ : & $1.43 \mathrm{E}-01$ & $9.48 \mathrm{E}-01$ & $\mathrm{Ca}$ \\
\hline & Total weight of leached impurity $(\mu \mathrm{g})$ : & 7.15 & 49.77 & 56.92 \\
\hline \multirow{2}{*}{ Al } & Measured concentration $(\mu \mathrm{g} / \mathrm{ml})$ : & $5.18 \mathrm{E}-02$ & $5.42 E-02$ & Al \\
\hline & Total weight of leached impurity $(\mu \mathrm{g})$ : & 2.59 & 2.85 & 5,44 \\
\hline \multirow{2}{*}{ Ti } & Measured concentration $(\mu \mathrm{g} / \mathrm{ml})$ : & $<8.00 \mathrm{E}-03$ & $<8.00 \mathrm{E}-03$ & $\mathrm{Ti}$ \\
\hline & Total weight of leached impurity $(\mu \mathrm{g})$ : & $<0.40$ & $<0.42$ & $<0.82$ \\
\hline \multirow{2}{*}{ v } & Measured concentration $(\mu \mathrm{g} / \mathrm{ml})$ : & $<2.00 \mathrm{E}-03$ & $<2.00 \mathrm{E}-03$ & $\mathrm{v}$ \\
\hline & Total weight of leached impurity $(\mu \mathrm{g})$ : & $<0.10$ & $<0.11$ & $<0.21$ \\
\hline
\end{tabular}

\section{Comments}

checked against official results of analyses for RMAL2014 by FCM on 7/23/2009

Fued C. Montyomeey
$7-23-09$

Date 
Data Report Form DRF-26A: Measurement of U Contamination or Impurities by Deconsolidation Leach

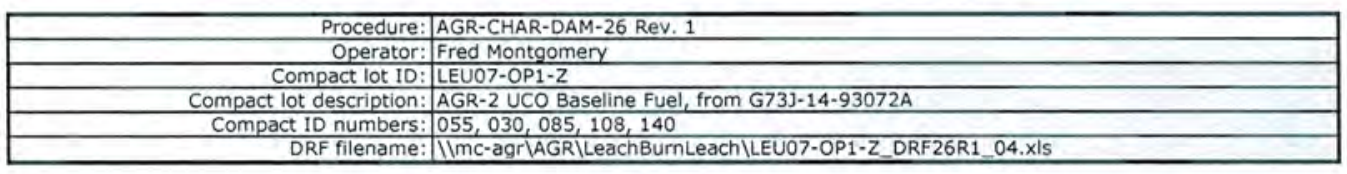

\begin{tabular}{|c|c|c|c|c|}
\hline \multicolumn{2}{|r|}{$\begin{aligned} & \text { Mean average weight uranium per particle }(g): \\
& \text { Uncertainty in mean average weight uranium per particle }(g):\end{aligned}$} & \multicolumn{3}{|c|}{$\frac{3.93 E-04}{6.56 E-07}$} \\
\hline & & First Leach & Second Leach & Total \\
\hline & Deconsolidation-leach solution ID: & L09060801 & L09061101 & \\
\hline & Number of compacts: & & & \\
\hline & Total volume of leach solution $(\mathrm{ml})$ : & 140.0 & 113.0 & \\
\hline & & & & \\
\hline & $\frac{\text { Radiochemical laboratory analysis number: }}{\text { Measured uranium concentration }(\mu \mathrm{g} / \mathrm{ml}):}$ & $\frac{1999-016}{287 E+00}$ & $\frac{1999-021}{1.69 E-01}$ & \\
\hline & & $\frac{2.87 E+00}{2.87 E-01}$ & $\frac{1.69 \mathrm{E}-01}{1.69 \mathrm{E}-02}$ & \\
\hline & Weight uranium leached $(g)$ : & $4.02 E-04$ & $1.91 \mathrm{E}-05$ & $4.21 \mathrm{E}-04$ \\
\hline & Uncertainty in weight uranium leached (g): & $4.02 E-05$ & $1.91 \mathrm{E}-06$ & $4.03 \mathrm{E}-05$ \\
\hline & Effective number of exposed kernels: & 1.0 & 0.0 & 1.1 \\
\hline & Uncertainty in effective number of exposed kernels: & 0.1 & 0.0 & 0.1 \\
\hline \multirow{5}{*}{$\mathrm{Fe}$} & Measured concentration of impurity in sample $(\mu \mathrm{g} / \mathrm{mll}):$ & $<4.13 \mathrm{E}-02$ & $<4.12 \mathrm{E}-02$ & $\mathrm{Fe}$ \\
\hline & Uncorrected weight of impurity in sample $(\mu \mathrm{g})$ : & $<5.78$ & $<4.66$ & $<10.44$ \\
\hline & Weight of impurity in blank $(\mu g)$ : & $<6.84$ & $<4.90$ & \\
\hline & Minimum corrected weight of impurity in sample $(\mu \mathrm{g})$ : & 0.00 & 0.00 & 0.00 \\
\hline & Maximum corrected weight of impurity in sample $(\mu \mathrm{g})$ : & 5.78 & 4.66 & 10.44 \\
\hline \multirow{4}{*}{$\mathrm{Cr}$} & Measured concentration of impurity in sample $(\mathrm{\mu g} / \mathrm{ml})$; & $1.02 \mathrm{E}-02$ & $7.95 \mathrm{E}-03$ & $\mathrm{Cr}$ \\
\hline & Uncorrected weight of impurity in sample $(\mu g)$ : & 1.43 & 0.90 & 2.33 \\
\hline & $\begin{array}{r}\text { Weight of impurity in blank }(\mu g) \text { : } \\
\text { Minimum corrected weight of impurity in sample }(\mu g):\end{array}$ & $<0.33$ & $<0.24$ & 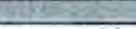 \\
\hline & $\begin{array}{l}\text { Minimum corrected weight of impurity in sample }(\mu g) \text { : } \\
\text { Maximum corrected weight of impurity in sample }(\mu g) \text { : }\end{array}$ & $\begin{array}{l}1.10 \\
1.43 \\
\end{array}$ & 0.66 & $\frac{1.76}{2.33}$ \\
\hline \multirow{5}{*}{$\mathrm{Mn}$} & Measured concentration of impurity in sample $(\mathrm{\mu g} / \mathrm{ml})$ : & $<1.93 \mathrm{E}-03$ & $<1.91 \mathrm{E}-03$ & $\mathrm{Mn}$ \\
\hline & Uncorrected weight of impurity in sample $(\mu g)$ : & $<0.27$ & $<0.22$ & $<0.49$ \\
\hline & Weight of impurity in blank $(\mu g)$ : & $<0.32$ & $<0.23$ & ons \\
\hline & Minimum corrected weight of impurity in sample $(\mu \mathrm{g})$ : & 0.00 & 0.00 & 0.00 \\
\hline & Maximum corrected weight of impurity in sample $(\mu \mathrm{g})$ : & 0.27 & 0.22 & 0.49 \\
\hline \multirow{5}{*}{ Co } & Measured concentration of impurity in sample $(\mathrm{\mu g} / \mathrm{ml})$ : & $<1.62 \mathrm{E}-03$ & $<1.62 \mathrm{E}-03$ & Co \\
\hline & Uncorrected weight of impurity in sample $(\mu \mathrm{g})$ : & $<0.23$ & $<0.18$ & $<0.41$ \\
\hline & Weight of impurity in blank $(\mu \mathrm{g})$ : & $<0.27$ & $<0.19$ & \\
\hline & Minimum corrected weight of impurity in sample $(\mu \mathrm{g})$ : & 0.00 & 0.00 & 0.00 \\
\hline & Maximum corrected weight of impurity in sample $(\mu g)$ : & 0.23 & 0.18 & 0.41 \\
\hline \multirow{5}{*}{$\mathrm{Ni}$} & Measured concentration of impurity in sample $(\mu \mathrm{g} / \mathrm{ml})$ : & $<8.00 \mathrm{E}-03$ & $<8.00 \mathrm{E}-03$ & $\mathrm{Ni}$ \\
\hline & Uncorrected weight of impurity in sample $(\mu \mathrm{g})$ : & $<1.12$ & $<0.90$ & $<2.02$ \\
\hline & Weight of impurity in blank $(\mathrm{\mu g})$ : & $<1.33$ & $<0.95$ & \\
\hline & Minimum corrected weight of impurity in sample $(\mu \mathrm{g})$ : & 0.00 & 0.00 & 0.00 \\
\hline & Maximum corrected weight of impurity in sample $(\mu \mathrm{gg})$ : & 1,12 & 0.90 & 2.02 \\
\hline \multirow{5}{*}{$\mathrm{Ca}$} & Measured concentration of impurity in sample $(\mu \mathrm{g} / \mathrm{mi})$ : & $<1.00 \mathrm{E}-01$ & $2.77 \mathrm{E}-01$ & $\mathrm{Ca}$ \\
\hline & Uncorrected weight of impurity in sample $(\mu \mathrm{g})$ : & $<14.00$ & 31.30 & $<45.30$ \\
\hline & Weight of impurity in blank $(\mu \mathrm{g})$ : & $<16.60$ & $<11.90$ & \\
\hline & Minimum corrected weight of impurity in sample $(\mu \mathrm{g})$ : & 0.00 & 19.40 & 19.40 \\
\hline & Maximum corrected weight of impurity in sample $(\mu g)$ : & 14.00 & 31.30 & 45.30 \\
\hline \multirow{5}{*}{ Al } & Measured concentration of impurity in sample $(\mu \mathrm{g} / \mathrm{ml})$ : & $4.17 E-01$ & $1.98 \mathrm{E}-01$ & Al \\
\hline & Uncorrected weight of impurity in sample $(\mu g)$ : & 58.38 & 22.37 & 80.75 \\
\hline & Weight of impurity in blank $(\mu \mathrm{g})$ : & 4.80 & 4.78 & \\
\hline & Minimum corrected weight of impurity in sample $(\mu \mathrm{g})$ : & 53.58 & 17.59 & 71.17 \\
\hline & Maximum corrected weight of impurity in sample $(\mu g)$ : & 53.58 & 17.59 & 71.17 \\
\hline \multirow{5}{*}{$\mathrm{Ti}$} & Measured concentration of impurity in sample $(\mathrm{\mu g} / \mathrm{ml})$ : & $2.89 \mathrm{E}-02$ & $3.19 \mathrm{E}-02$ & \\
\hline & Uncorrected weight of impurity in sample $(\mu \mathrm{g})$ : & 4.05 & 3.60 & 7.65 \\
\hline & Weight of impurity in blank $(\mu \mathrm{g})$ : & $<1.33$ & $<0.95$ & \\
\hline & Minimum corrected weight of impurity in sample $(\mu g)$ : & 2.72 & 2.65 & 5.37 \\
\hline & Maximum corrected weight of impurity in sample $(\mu g)$ : & 4.05 & 3.60 & 7.65 \\
\hline \multirow{5}{*}{$\mathbf{v}$} & Measured concentration of impurity in sample $(\mu \mathrm{g} / \mathrm{ml})$ : & $2.44 \mathrm{E}-01$ & $7.62 E-02$ & $\mathrm{v}$ \\
\hline & Uncorrected weight of impurity in sample $(\mu \mathrm{g})$ : & 34.16 & 8.61 & 42.77 \\
\hline & Weight of impurity in blank $(\mu \mathrm{g})$ : & $<0.33$ & $<0.24$ & \\
\hline & Minimum corrected weight of impurity in sample $(\mu g)$ : & 33.83 & 8.37 & 42.20 \\
\hline & Maximum corrected weight of impurity in sample $(\mu g)$ : & 34.16 & 8.61 & 42.77 \\
\hline
\end{tabular}

Comments

Checked against official results of RMAL1999 by FCM on 7/14/2009

$\frac{7 \text { red c. Montgo meey }}{\text { operator }}$
$7-23-09$ 
Data Report Form DRF-26A: Measurement of U Contamination or Impurities by Deconsolidation Leach

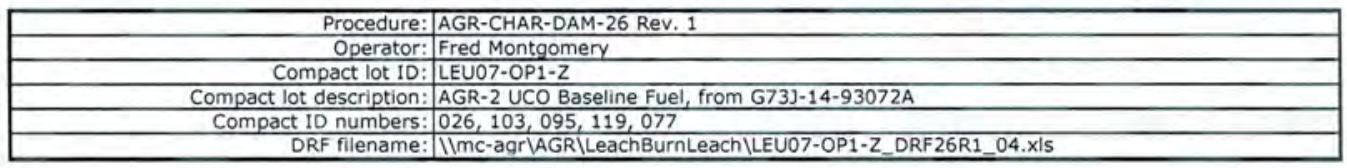

\begin{tabular}{|c|c|c|c|c|}
\hline \multicolumn{2}{|c|}{$\begin{array}{c}\text { Mean average weight uranium per particle }(g) \text { : } \\
\text { Uncertainty in mean average weight uranium per particle }(q):\end{array}$} & \multicolumn{3}{|c|}{$\begin{array}{l}3.93 \mathrm{E}-04 \\
6.56 \mathrm{E}-07\end{array}$} \\
\hline & & First Leach & Second Leach & Total \\
\hline & Deconsolidation-leach solution ID: & L09060802 & L09061102 & \\
\hline & Number of compacts: & & & \\
\hline & Total volume of leach solution $(\mathrm{ml})$ : & 144.0 & 118.0 & \\
\hline & Radiochemical laboratory analysis number: & $1999-017$ & $1999-022$ & \\
\hline & Measured uranium concentration $(\mathrm{\mu g} / \mathrm{ml})$ : & $2.90 E+00$ & $1.67 \mathrm{E}-01$ & \\
\hline & Uncertainty in uranium concentration $(\mathrm{\mu g} / \mathrm{ml})$ : & $2.90 \mathrm{E}-01$ & $1.67 \mathrm{E}-02$ & \\
\hline & Weight uranium leached $(g)$ : & $4.18 \mathrm{E}-04$ & $1.97 \mathrm{E}-05$ & 4.37E-04 \\
\hline & Uncertainty in weight uranium leached $(g)$ : & $4.18 \mathrm{E}-05$ & $1.97 E-06$ & $4.18 E-05$ \\
\hline & Effective number of exposed kernels: & 1.1 & 0.1 & 1.1 \\
\hline & Uncertainty in effective number of exposed kernels: & 0.1 & 0.0 & 0.1 \\
\hline \multirow{5}{*}{$\mathrm{Fe}$} & Measured concentration of impurity in sample $(\mu \mathrm{g} / \mathrm{ml})$ : & $<4.12 \mathrm{E}-02$ & $<4.12 \mathrm{E}-02$ & $\mathrm{Fe}$ \\
\hline & Uncorrected weight of impurity in sample $(\mu \mathrm{g})$ : & $<5.93$ & $<4.86$ & $<10.79$ \\
\hline & Weight of impurity in blank $(\mu \mathrm{g})$ : & $<6.84$ & $<4.90$ & \\
\hline & Minimum corrected weight of impurity in sample $(\mu \mathrm{g})$ : & 0.00 & 0.00 & 0.00 \\
\hline & Maximum corrected weight of impurity in sample ( $\mu \mathrm{g})$ : & 5.93 & 4.86 & 10.79 \\
\hline \multirow{5}{*}{$\mathrm{Cr}$} & Measured concentration of impurity in sample $(\mu \mathrm{g} / \mathrm{ml})$ : & $7.63 E-03$ & $8.60 \mathrm{E}-03$ & $\mathrm{Cr}$ \\
\hline & Uncorrected weight of impurity in sample $(\mu \mathrm{g})$ : & 1.10 & 1.01 & 2.11 \\
\hline & Weight of impurity in blank $(\mu \mathrm{g})$ : & $<0.33$ & $<0.24$ & \\
\hline & Minimum corrected weight of impurity in sample $(\mu \mathrm{g})$ : & 0.77 & 0.78 & 1.54 \\
\hline & Maximum corrected weight of impurity in sample $(\mu \mathrm{g})$ : & 1.10 & 1.01 & 2.11 \\
\hline \multirow{5}{*}{ Mn } & Measured concentration of impurity in sample $(\mu \mathrm{g} / \mathrm{ml})$ : & $<1.91 \mathrm{E}-03$ & $<1.91 \mathrm{E}-03$ & $\mathrm{Mn}$ \\
\hline & Uncorrected weight of impurity in sample $(\mu g)$ : & $<0.28$ & $<0.23$ & $<0.50$ \\
\hline & Weight of impurity in blank $(\mu \mathrm{g})$ : & $<0.32$ & $<0.23$ & \\
\hline & Minimum corrected weight of impurity in sample $(\mu \mathrm{g})$ : & 0.00 & 0.00 & 0.00 \\
\hline & Maximum corrected weight of impurity in sample $(\mu g)$ : & 0.28 & 0.23 & 0.50 \\
\hline \multirow{5}{*}{ co } & Measured concentration of impurity in sample $(\mu \mathrm{g} / \mathrm{ml})$ : & $<1.62 \mathrm{E}-03$ & $<1.62 \mathrm{E}-03$ & Co \\
\hline & Uncorrected weight of impurity in sample $(\mu \mathrm{g})$ : & $<0.23$ & $<0.19$ & $<0.42$ \\
\hline & Weight of impurity in blank $(\mu \mathrm{g})$ : & $<0.27$ & $<0.19$ & \\
\hline & Minimum corrected weight of impurity in sample $(\mu \mathrm{g})$ : & 0.00 & 0.00 & 0.00 \\
\hline & Maximum corrected weight of impurity in sample $(\mathrm{\mu g})$ : & 0.23 & 0.19 & 0.42 \\
\hline \multirow{5}{*}{$\mathrm{Ni}$} & Measured concentration of impurity in sample $(\mathrm{\mu g} / \mathrm{ml})$ : & $<8.00 \mathrm{E}-03$ & $<8.00 \mathrm{E}-03$ & $\mathrm{Ni}$ \\
\hline & Uncorrected weight of impurity in sample $(\mu \mathrm{g})$ : & $<1.15$ & $<0.94$ & $<2.10$ \\
\hline & Weight of impurity in blank $(\mu \mathrm{g})$ : & $<1.33$ & $<0.95$ & \\
\hline & Minimum corrected weight of impurity in sample $(\mathrm{\mu g})$ : & 0.00 & 0.00 & 0.00 \\
\hline & Maximum corrected weight of impurity in sample $(\mu \mathrm{\mu g})$ : & 1.15 & 0.94 & 2.10 \\
\hline \multirow{5}{*}{$\mathrm{Ca}$} & Measured concentration of impurity in sample $(\mu \mathrm{g} / \mathrm{ml})$ : & $<1.00 \mathrm{E}-01$ & $2.10 \mathrm{E}-01$ & $\mathrm{Ca}$ \\
\hline & Uncorrected weight of impurity in sample $(\mu \mathrm{g})$ : & $<14.40$ & 24.78 & $<39.18$ \\
\hline & Weight of impurity in blank $(\mu g)$ : & $<16.60$ & $<11.90$ & \\
\hline & Minimum corrected weight of impurity in sample $(\mathrm{\mu g})$ : & 0.00 & 12.88 & 12.88 \\
\hline & Maximum corrected weight of impurity in sample $(\mu \mathrm{g})$ : & 14.40 & 24.78 & 39.18 \\
\hline \multirow{5}{*}{ Al } & Measured concentration of impurity in sample $(\mu \mathrm{g} / \mathrm{ml})$ : & $3.76 \mathrm{E}-01$ & $1.72 \mathrm{E}-01$ & Al \\
\hline & Uncorrected weight of impurity in sample $(\mu \mathrm{g})$ : & 54.14 & 20.30 & 74.44 \\
\hline & Weight of impurity in blank $(\mu g)$ : & 4.80 & 4.78 & \\
\hline & Minimum corrected weight of impurity in sample $(\mathrm{gg})$ : & 49.35 & $\frac{15.51}{1.54}$ & 64.86 \\
\hline & Maximum corrected weight of impurity in sample $(\mu \mathrm{g})$ : & 49.35 & 15.51 & 64.86 \\
\hline \multirow{5}{*}{$\mathrm{Ti}$} & Measured concentration of impurity in sample $(\mu \mathrm{g} / \mathrm{ml})$ : & $2.43 E-02$ & $3.11 \mathrm{E}-02$ & $\mathrm{Ti}$ \\
\hline & Uncorrected weight of impurity in sample $(\mu \mathrm{g})$ : & 3.50 & 3.67 & 7.17 \\
\hline & Weight of impurity in blank $(\mu g)$ : & $<1.33$ & $<0.95$ & \\
\hline & Minimum corrected weight of impurity in sample $(\mu g)$ : & 2.17 & 2.72 & 4.89 \\
\hline & Maximum corrected weight of impurity in sample $(\mu \mathrm{g})$ : & 3.50 & 3.67 & 7.17 \\
\hline \multirow{4}{*}{ v } & Measured concentration of impurity in sample $(\mu \mathrm{g} / \mathrm{ml})$ : & $2.33 \mathrm{E}-01$ & $7.22 \mathrm{E}-02$ & $\mathrm{v}$ \\
\hline & Uncorrected weight of impurity in sample $(\mu g)$ : & $\frac{33.55}{1.32}$ & 8.52 & 42.07 \\
\hline & $\begin{array}{l}\text { Weight of impurity in blank }(\mu g) \text { : } \\
\text { Minimum corrected weight of impurity in sample }(\mu g) \text { : }\end{array}$ & $<0.33$ & $<0.24$ & \\
\hline & $\begin{array}{l}\text { Minimum corrected weight of impurity in sample }(\mu g) \text { : } \\
\text { Maximum corrected weight of impurity in sample }(\mu g) \text { : }\end{array}$ & $\frac{33.22}{33.55}$ & $\frac{8.28}{8.52}$ & $\begin{array}{l}41.50 \\
42.07\end{array}$ \\
\hline
\end{tabular}

comments 
Data Report Form DRF-26A: Measurement of U Contamination or Impurities by Deconsolidation Leach

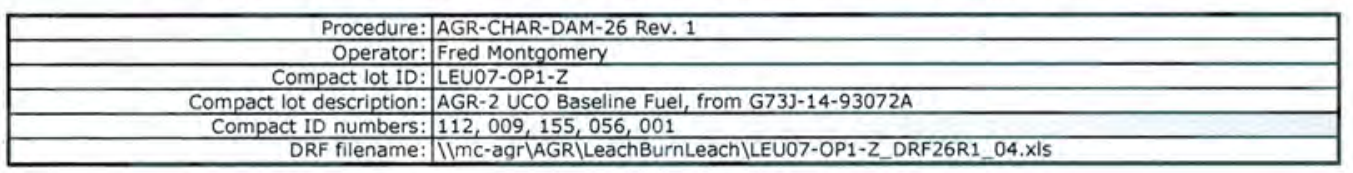

\begin{tabular}{|c|c|c|c|c|}
\hline & $\begin{array}{l}\text { Mean average weight uranium per particle (g): } \\
\text { Uncertainty in mean average weight uranium per particle (g): }\end{array}$ & & $\frac{3.93 E-04}{6.56 E-07}$ & \\
\hline & & First Leach & Second Leach & Total \\
\hline & Deconsolidation-leach solution ID: & L09060803 & L09061103 & \\
\hline & Number of compacts: & & & \\
\hline & Total volume of leach solution $(\mathrm{ml})$ : & 140.0 & 119.0 & \\
\hline & Radiochemical laboratory analysis numberi & & & \\
\hline & $\frac{\text { Radiochemical laboratory analysis number: }}{\text { Measured uranium concentration }(\mu \mathrm{g} / \mathrm{ml}):}$ & $\frac{1999-018}{3.90 \mathrm{E}-02}$ & $\frac{1999-023}{1.31 \mathrm{E}-02}$ & \\
\hline & Uncertainty in uranium concentration $(\mu \mathrm{g} / \mathrm{ml})$ : & $3.90 \mathrm{E}-03$ & $1.31 \mathrm{E}-03$ & \\
\hline & Weight uranium leached $(g):$ & $5.46 \mathrm{E}-06$ & $1.56 \mathrm{E}-06$ & $7.02 E-06$ \\
\hline & Uncertainty in weight uranium leached $(g)$ : & 5.47E-07 & $1.56 \mathrm{E}-07$ & $5.68 \mathrm{E}-07$ \\
\hline & Effective number of exposed kernels: & 0.0 & 0.0 & 0.0 \\
\hline & Uncertainty in effective number of exposed kernels: & 0.0 & 0.0 & 0.0 \\
\hline & Measured concentration of impurity in sample $(\mathrm{\mu g} / \mathrm{ml})$ : & $<4.12 \mathrm{E}-02$ & $<4.12 \mathrm{E}-02$ & \\
\hline & Uncorrected weight of impurity in sample $(\mu \mathrm{g})$ : & $<5.77$ & $<4.90$ & $<10.67$ \\
\hline $\mathbf{F e}$ & Weight of impurity in blank $(\mu g):$ & $<6.84$ & $<4.90$ & \\
\hline & Minimum corrected weight of impurity in sample $(\mu \mathrm{g})$ : & 0.00 & 0.00 & 0.00 \\
\hline & Maximum corrected weight of impurity in sample $(\mu \mathrm{g})$ : & 5.77 & 4.90 & 10.67 \\
\hline & Measured concentration of impurity in sample $(\mu \mathrm{g} / \mathrm{ml})$ : & $5.28 \mathrm{E}-03$ & $9.38 \mathrm{E}-03$ & \\
\hline & Uncorrected weight of impurity in sample $(\mu \mathrm{g})$ : & 0.74 & 1.12 & 1.86 \\
\hline Cr & Weight of impurity in blank $(\mu \mathrm{g})$ : & $<0.33$ & $<0.24$ & \\
\hline & Minimum corrected weight of impurity in sample $(\mu \mathrm{g})$ : & 0.41 & 0.88 & 1.29 \\
\hline & Maximum corrected weight of impurity in sample $(\mu g)$ : & 0.74 & 1.12 & 1.86 \\
\hline & Measured concentration of impurity in sample $(\mu \mathrm{g} / \mathrm{ml}):$ & $<1.91 \mathrm{E}-03$ & $<1.91 \mathrm{E}-03$ & $\mathrm{Mn}$ \\
\hline & Uncorrected weight of impurity in sample $(\mu \mathrm{g})$ : & $<0.27$ & $<0.23$ & $<0.49$ \\
\hline Mn & Weight of impurity in blank ( $\mu \mathrm{g})$ : & $<0.32$ & $<0.23$ & \\
\hline & Minimum corrected weight of impurity in sample $(\mu \mathrm{g})$ : & 0.00 & 0.00 & 0.00 \\
\hline & Maximum corrected weight of impurity in sample $(\mu g)$ : & 0.27 & 0.23 & 0.49 \\
\hline & Measured concentration of impurity in sample $(\mu \mathrm{g} / \mathrm{ml})$ : & $<1.62 \mathrm{E}-03$ & $<1.62 \mathrm{E}-03$ & Co \\
\hline & Uncorrected weight of impurity in sample $(\mu \mathrm{g})$ : & $<0.23$ & $<0.19$ & $<0.42$ \\
\hline Co & Weight of impurity in blank $(\mu \mathrm{g})$ : & $<0.27$ & $<0.19$ & \\
\hline & Minimum corrected weight of impurity in sample $(\mu g)$ : & 0.00 & 0.00 & 0.00 \\
\hline & Maximum corrected weight of impurity in sample $(\mu \mathrm{g})$ : & 0.23 & 0.19 & 0.42 \\
\hline & Measured concentration of impurity in sample $(\mu \mathrm{g} / \mathrm{ml})$ : & $<8.00 \mathrm{E}-03$ & $<8.00 \mathrm{E}-03$ & $\mathrm{Ni}$ \\
\hline & Uncorrected weight of impurity in sample $(\mu \mathrm{g})$ : & $<1.12$ & $<0.95$ & $<2.07$ \\
\hline $\mathrm{Ni}$ & Weight of impurity in blank $(\mu \mathrm{g})$ : & $<1.33$ & $<0.95$ & \\
\hline & Minimum corrected weight of impurity in sample $(\mu g)$ : & 0.00 & 0.00 & 0.00 \\
\hline & Maximum corrected weight of impurity in sample $(\mu g)$ : & 1.12 & 0.95 & 2.07 \\
\hline & Measured concentration of impurity in sample $(\mu \mathrm{g} / \mathrm{ml})$ : & $2.66 \mathrm{E}-01$ & $<1.00 \mathrm{E}-01$ & $\mathrm{Ca}$ \\
\hline & Uncorrected weight of impurity in sample $(\mu \mathrm{g})$ : & 37.24 & $<11.90$ & $<49.14$ \\
\hline Ca & Weight of impurity in blank $(\mu g)$ : & $<16.60$ & $<11.90$ & \\
\hline & Minimum corrected weight of impurity in sample $(\mu g)$ : & 20.64 & 0.00 & 20.64 \\
\hline & Maximum corrected weight of impurity in sample $(\mu \mathrm{g})$ : & 37.24 & 11.90 & 49.14 \\
\hline & Measured concentration of impurity in sample $(\mathrm{\mu g} / \mathrm{ml})$ : & $3.61 \mathrm{E}-01$ & $1.27 \mathrm{E}-01$ & Al \\
\hline & Uncorrected weight of impurity in sample $(\mu g)$ : & 50.54 & 15.11 & 65.65 \\
\hline Al & Weight of impurity in blank $(\mu \mathrm{g})$ : & 4.80 & 4.78 & \\
\hline & Minimum corrected weight of impurity in sample $(\mu g)$ : & 45.74 & 10.33 & 56.07 \\
\hline & Maximum corrected weight of impurity in sample $(\mu g)$ : & 45.74 & 10.33 & 56.07 \\
\hline & Measured concentration of impurity in sample $(\mu \mathrm{g} / \mathrm{ml})$ : & $1.72 \mathrm{E}-02$ & $2.44 E-02$ & $\mathbf{T i}$ \\
\hline & Uncorrected weight of impurity in sample $(\mu g)$ : & 2.41 & 2.90 & 5.31 \\
\hline $\mathrm{Ti}$ & Weight of impurity in blank $(\mu \mathrm{g})$ : & $<1.33$ & $<0.95$ & \\
\hline & Minimum corrected weight of impurity in sample $(\mu g)$ : & $\frac{1.08}{2.14}$ & $\frac{1.95}{200}$ & $\frac{3.03}{5.31}$ \\
\hline & Maximum corrected weight of impurity in sample $(\mu g)$ : & 2.41 & 2.90 & 5.31 \\
\hline & Measured concentration of impurity in sample $(\mathrm{\mu g} / \mathrm{ml})$ : & $2.16 \mathrm{E}-01$ & $8.14 \mathrm{E}-02$ & $\mathbf{v}$ \\
\hline & Uncorrected weight of impurity in sample $(\mu g)$ : & 30.24 & 9.69 & 39.93 \\
\hline v & Weight of impurity in blank $(\mu \mathrm{g})$ : & $<0.33$ & $\leq 0.24$ & \\
\hline & Minimum corrected weight of impurity in sample $(\mu g)$ : & 29.91 & 9.45 & $\frac{39.36}{30.93}$ \\
\hline & Maximum corrected weight of impurity in sample $(\mu g)$ : & 30.24 & 9.69 & 39.93 \\
\hline
\end{tabular}

\section{Comments}


Data Report Form DRF-26A: Measurement of U Contamination or Impurities by Deconsolidation Leach

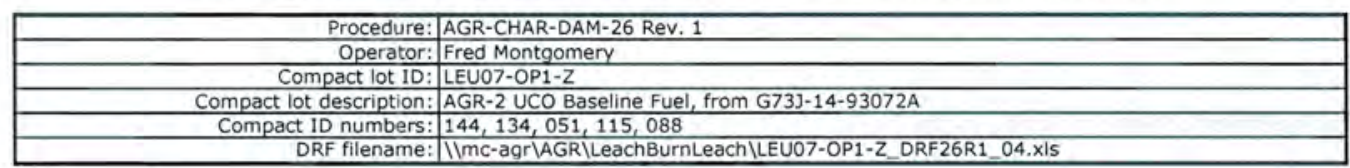

\begin{tabular}{|c|c|c|c|c|}
\hline \multicolumn{2}{|c|}{$\begin{array}{r}\text { Mean average weight uranium per particle }(\mathrm{g}) \text { : } \\
\text { Uncertainty in mean average weight uranium per particle (a): }\end{array}$} & \multicolumn{3}{|c|}{$\begin{array}{l}3.93 \mathrm{E}-04 \\
6.56 \mathrm{E}-07 \\
\end{array}$} \\
\hline & & First Leach & Second Leach & Total \\
\hline & Deconsolidation-leach solution ID: & L09060804 & $L 09061104$ & \\
\hline & Number of compacts: & & & \\
\hline & Total volume of leach solution $(\mathrm{ml})$ : & 140.0 & 115.0 & \\
\hline & Radiochemical laboratory analysis number: & 1999-019 & 1999-024 & \\
\hline & Measured uranium concentration $(\mu \mathrm{g} / \mathrm{ml})$ : & $3.90 \mathrm{E}-02$ & $1.56 \mathrm{E}-02$ & \\
\hline & Uncertainty in uranium concentration $(\mathrm{\mu g} / \mathrm{ml})$ : & $3.90 \mathrm{E}-03$ & $1.56 \mathrm{E}-03$ & \\
\hline & Weight uranium leached $(g)$ : & $5.46 \mathrm{E}-06$ & $1.79 \mathrm{E}-06$ & $7.25 \mathrm{E}-06$ \\
\hline & Uncertainty in weight uranium leached $(g)$ : & $5.47 E-07$ & $1.80 \mathrm{E}-07$ & $5.75 \mathrm{E}-07$ \\
\hline & Effective number of exposed kernels: & 0.0 & 0.0 & 0.0 \\
\hline & Uncertainty in effective number of exposed kernels: & 0.0 & 0.0 & 0.0 \\
\hline & Measured concentration of impurity in sample $(\mathrm{\mu g} / \mathrm{ml})$ : & $<4.12 \mathrm{E}-02$ & $<4.12 \mathrm{E}-02$ & \\
\hline \multirow{4}{*}{$\mathrm{Fe}$} & Uncorrected weight of impurity in sample $(\mu g)$ : & $<5.77$ & $<4.74$ & $\frac{r e}{<10.51}$ \\
\hline & Weight of impurity in blank $(\mu g)$ : & $<6.84$ & $<4.90$ & \\
\hline & Minimum corrected weight of impurity in sample $(\mu g)$ : & 0.00 & 0.00 & 0.00 \\
\hline & Maximum corrected weight of impurity in sample $(\mu q)$ : & 5.77 & 4.74 & 10.51 \\
\hline \multirow{5}{*}{$\mathrm{Cr}$} & Measured concentration of impurity in sample $(\mathrm{\mu g} / \mathrm{ml})$ : & $6.36 \mathrm{E}-03$ & $8.78 \mathrm{E}-03$ & $\mathrm{Cr}$ \\
\hline & Uncorrected weight of impurity in sample $(\mu g)$ : & 0.89 & 1.01 & 1.90 \\
\hline & Weight of impurity in blank $(\mu \mathrm{g})$ : & $<0.33$ & $<0.24$ & \\
\hline & Minimum corrected weight of impurity in sample $(\mu g)$ : & 0.56 & 0.77 & 1.33 \\
\hline & Maximum corrected weight of impurity in sample $(\mu g)$ : & 0.89 & 1.01 & 1.90 \\
\hline \multirow{5}{*}{$\mathrm{Mr}$} & Measured concentration of impurity in sample $(\mathrm{\mu g} / \mathrm{ml})$ : & $<1.91 \mathrm{E}-03$ & $<1.91 \mathrm{E}-03$ & $\mathrm{Mn}$ \\
\hline & Uncorrected weight of impurity in sample $(\mu g)$ : & $<0.27$ & $<0.22$ & $<0.49$ \\
\hline & Weight of impurity in blank $(\mu \mathrm{g})$ : & $<0.32$ & $<0.23$ & \\
\hline & Minimum corrected weight of impurity in sample $(\mu g)$ : & 0.00 & 0.00 & 0.00 \\
\hline & Maximum corrected weight of impurity in sample $(\mu \mathrm{g})$ : & 0.27 & 0.22 & 0.49 \\
\hline \multirow{5}{*}{ Co } & Measured concentration of impurity in sample $(\mu \mathrm{g} / \mathrm{ml})$ : & $<1.62 \mathrm{E}-03$ & $<1.62 \mathrm{E}-03$ & Co \\
\hline & Uncorrected weight of impurity in sample $(\mu g)$ : & $<0.23$ & $<0.19$ & $<0.41$ \\
\hline & Weight of impurity in blank $(\mu g)$ : & $<0.27$ & $<0.19$ & \\
\hline & Minimum corrected weight of impurity in sample $(\mu g)$ : & 0.00 & 0.00 & 0.00 \\
\hline & Maximum corrected weight of impurity in sample $(\mu g)$ : & 0.23 & 0.19 & 0.41 \\
\hline \multirow{5}{*}{$\mathrm{Ni}$} & Measured concentration of impurity in sample $(\mu \mathrm{g} / \mathrm{ml})$ : & $<8.00 \mathrm{E}-03$ & $<8.00 \mathrm{E}-03$ & $\mathrm{Ni}$ \\
\hline & Uncorrected weight of impurity in sample $(\mu g)$ : & $<1.12$ & $<0.92$ & $<2.04$ \\
\hline & Weight of impurity in blank $(\mu \mathrm{g})$ : & $<1.33$ & $<0.95$ & \\
\hline & Minimum corrected weight of impurity in sample $(\mu \mathrm{g})$ : & 0.00 & 0.00 & 0.00 \\
\hline & Maximum corrected weight of impurity in sample $(\mu g)$ : & 1.12 & 0.92 & 2.04 \\
\hline \multirow{5}{*}{$\mathrm{Ca}$} & Measured concentration of impurity in sample $(\mathrm{\mu g} / \mathrm{ml})$ : & $<1.00 \mathrm{E}-01$ & $<1.00 \mathrm{E}-01$ & $\mathrm{Ca}$ \\
\hline & Uncorrected weight of impurity in sample $(\mu \mathrm{g})$ : & $<14.00$ & $<11.50$ & $<25.50$ \\
\hline & Weight of impurity in blank $(\mu \mathrm{g})$ : & $<16.60$ & $<11.90$ & \\
\hline & Minimum corrected weight of impurity in sample $(\mu \mathrm{g})$ : & 0.00 & 0.00 & 0.00 \\
\hline & Maximum corrected weight of impurity in sample $(\mu g)$ : & 14.00 & 11.50 & 25.50 \\
\hline \multirow{5}{*}{ Al } & Measured concentration of impurity in sample $(\mathrm{\mu g} / \mathrm{ml})$ : & $3.70 E-01$ & $1.89 \mathrm{E}-01$ & Al \\
\hline & Uncorrected weight of impurity in sample $(\mu \mathrm{g})$ : & 51.80 & 21.74 & 73.54 \\
\hline & Weight of impurity in blank $(\mu \mathrm{g})$ : & 4.80 & 4.78 & \\
\hline & Minimum corrected weight of impurity in sample $(\mu g)$ : & 47.00 & 16.95 & 63.95 \\
\hline & Maximum corrected weight of impurity in sample $(\mu \mathrm{g})$ : & 47.00 & 16.95 & 63.95 \\
\hline \multirow{5}{*}{$\mathrm{Ti}$} & Measured concentration of impurity in sample $(\mathrm{\mu g} / \mathrm{ml})$ : & $2.20 \mathrm{E}-02$ & $3.08 \mathrm{E}-02$ & $\mathrm{Ti}$ \\
\hline & Uncorrected weight of impurity in sample $(\mu \mathrm{g})$ : & 3.08 & 3.54 & 6.62 \\
\hline & Weight of impurity in blank $(\mathrm{\mu g})$ : & $<1.33$ & $<0.95$ & \\
\hline & Minimum corrected weight of impurity in sample $(\mu \mathrm{g})$ : & 1.75 & 2.59 & 4.34 \\
\hline & Maximum corrected weight of impurity in sample $(\mu \mathrm{g})$ : & 3.08 & 3.54 & 6.62 \\
\hline \multirow{5}{*}{ v } & Measured concentration of impurity in sample $(\mathrm{\mu g} / \mathrm{ml})$ : & $2,17 \mathrm{E}-01$ & $7.34 \mathrm{E}-02$ & $\mathrm{v}$ \\
\hline & Uncorrected weight of impurity in sample $(\mu \mathrm{g})$ : & 30.38 & 8.44 & 38.82 \\
\hline & Weight of impurity in blank $(\mu \mathrm{g})$ : & $<0.33$ & $<0.24$ & 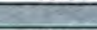 \\
\hline & Minimum corrected weight of impurity in sample $(\mu g)$ : & 30.05 & 8.20 & 38.25 \\
\hline & Maximum corrected weight of impurity in sample $(\mu \mathrm{g})$ : & 30.38 & 8.44 & 38.82 \\
\hline
\end{tabular}

comments

Checked against official results of RMAL1999 by FCM on 7/14/2009

$\frac{\text { Fied C. montgomuly }}{\text { operator }}-7-23-09$ Date


Data Report Form DRF-26A: Measurement of U Contamination or Impurities by Deconsolidation Leach

\begin{tabular}{|r|l|}
\hline Procedure: & AGR-CHAR-DAM-26 Rev. 1 \\
\hline Operator: & Fred Montgomery \\
\hline Compact lot ID: & LEU07-OP1-Z \\
\hline Compact lot description: & AGR-2 UCO Baseline Fuel, from G73J-14-93072A \\
\hline Compact ID numbers: & Deconsolidation Leach Blank \\
\hline DRF filename: & \Imc-agr\AGR\LeachBurnLeach \LEU07-OP1-Z_DRF26R1_04.xIs \\
\hline
\end{tabular}

\begin{tabular}{|r|c|}
\hline Mean average weight uranium per particle $(\mathrm{g}):$ & $3.93 \mathrm{E}-04$ \\
\hline Uncertainty in mean average weight uranium per particle $(\mathrm{g}):$ & $6.56 \mathrm{E}-07$ \\
\hline
\end{tabular}

\begin{tabular}{|c|c|c|c|c|}
\hline & & First Leach & Second Leach & Total \\
\hline & Deconsolidation-leach solution ID: & L09060805 & L09061105 & \\
\hline & Number of compacts: & \multicolumn{2}{|c|}{ None } & \\
\hline & Total volume of leach solution $(\mathrm{ml}):$ - & 166.0 & 119.0 & \\
\hline \multicolumn{4}{|c|}{ 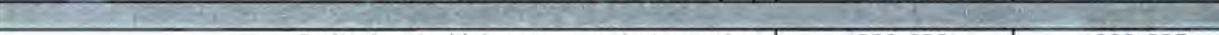 } & \\
\hline & Radiochemical laboratory analysis number: & $1999-020$ & $1999-025$ & \\
\hline & Measured uranium concentration $(\mu \mathrm{g} / \mathrm{ml})$ : & $<2.00 \mathrm{E}-04$ & $<2.00 E-04$ & \\
\hline \multicolumn{2}{|r|}{ Uncertainty in uranium concentration $(\mu \mathrm{g} / \mathrm{ml})$ : } & & & \\
\hline \multirow{2}{*}{\multicolumn{2}{|c|}{$\begin{array}{r}\text { Weight uranium leached }(\mathrm{g}): \\
\text { Uncertainty in weight uranium leached }(\mathrm{g}):\end{array}$}} & $<3.32 \mathrm{E}-08$ & $<2.38 \mathrm{E}-08$ & $<5.70 \mathrm{E}-08$ \\
\hline & & & & \\
\hline \multirow{2}{*}{\multicolumn{2}{|c|}{$\begin{array}{r}\text { Effective number of exposed kernels: } \\
\text { Uncertainty in effective number of exposed kernels: }\end{array}$}} & 0.0 & 0.0 & 0.0 \\
\hline & & & & \\
\hline \multirow{3}{*}{$\mathrm{Fe}$} & & & & \\
\hline & Measured concentration $(\mu \mathrm{g} / \mathrm{ml})$ : & $<4.12 \mathrm{E}-02$ & $<4.12 \mathrm{E}-02$ & $\mathrm{Fe}$ \\
\hline & Total weight of leached impurity $(\mu \mathrm{g})$ : & $<6.84$ & $<4.90$ & $<11.74$ \\
\hline \multirow{2}{*}{$\mathrm{Cr}$} & Measured concentration $(\mu \mathrm{g} / \mathrm{ml})$ : & $<2.00 \mathrm{E}-03$ & $<2.00 \mathrm{E}-03$ & $\mathrm{Cr}$ \\
\hline & Total weight of leached impurity $(\mu \mathrm{g})$ : & $<0.33$ & $<0.24$ & $<0.57$ \\
\hline \multirow{2}{*}{ Mn } & Measured concentration $(\mu \mathrm{g} / \mathrm{ml})$ : & $<1.91 \mathrm{E}-03$ & $<1.91 \mathrm{E}-03$ & Mn \\
\hline & Total weight of leached impurity $(\mu g)$ : & $<0.32$ & $<0.23$ & $<0.54$ \\
\hline \multirow{2}{*}{ Co } & Measured concentration $(\mu \mathrm{g} / \mathrm{ml})$ : & $<1.62 \mathrm{E}-03$ & $<1.62 \mathrm{E}-03$ & Co \\
\hline & Total weight of leached impurity $(\mu g)$ : & $<0.27$ & $<0.19$ & $<0.46$ \\
\hline \multirow{2}{*}{$\mathrm{Ni}$} & Measured concentration $(\mu \mathrm{g} / \mathrm{ml})$ : & $<8.00 \mathrm{E}-03$ & $<8.00 E-03$ & $\mathrm{Ni}$ \\
\hline & Total weight of leached impurity $(\mu g)$ : & $<1.33$ & $<0.95$ & $<2.28$ \\
\hline \multirow{2}{*}{$\mathrm{Ca}$} & Measured concentration $(\mu \mathrm{g} / \mathrm{ml})$ : & $<1.00 \mathrm{E}-01$ & $<1.00 \mathrm{E}-01$ & $\mathrm{Ca}$ \\
\hline & Total weight of leached impurity $(\mu \mathrm{g})$ : & $<16.60$ & $<11.90$ & $<28.50$ \\
\hline \multirow{2}{*}{ Al } & Measured concentration $(\mu \mathrm{g} / \mathrm{ml})$ : & $2.89 \mathrm{E}-02$ & $4.02 E-02$ & Al \\
\hline & Total weight of leached impurity $(\mu \mathrm{g})$ : & 4.80 & 4.78 & 9.58 \\
\hline \multirow{2}{*}{$\mathrm{Ti}$} & Measured concentration $(\mu \mathrm{g} / \mathrm{ml})$ : & $<8.00 \mathrm{E}-03$ & $<8.00 \mathrm{E}-03$ & $\mathrm{Ti}$ \\
\hline & Total weight of leached impurity $(\mu \mathrm{g})$ : & $<1.33$ & $<0.95$ & $<2.28$ \\
\hline \multirow{2}{*}{ v } & Measured concentration $(\mu \mathrm{g} / \mathrm{ml})$ : & $<2.00 \mathrm{E}-03$ & $<2.00 \mathrm{E}-03$ & $\mathbf{V}$ \\
\hline & Total weight of leached impurity $(\mu \mathrm{g})$ : & $<0.33$ & $<0.24$ & $<0.57$ \\
\hline
\end{tabular}

\section{Comments}

\section{Feed c. Montgomeun}


Data Report Form DRF-26B: Measurement of SIC Burn-Leach Defects or Impurities by Burn-Leach

\begin{tabular}{|c|c|}
\hline $\begin{array}{rr} & \text { Procedure: } \\
& \text { Operator: } \\
\end{array}$ & $\begin{array}{l}\text { AGR-CHAR-DAM-26 Rev. } 1 \\
\text { Fred Montgomery }\end{array}$ \\
\hline Compact lot ID: & LEU07-OP1-Z \\
\hline Compact lot description: & AGR-2 UCO Baseline Fuel, from G73J-14-93072A \\
\hline Compact ID numbers: & $055,030,085,108,140$ \\
\hline ename & PP1-Z_DRF26R1_04.xls \\
\hline
\end{tabular}

\begin{tabular}{|c|c|c|c|c|}
\hline \multicolumn{2}{|r|}{\begin{tabular}{|c|} 
Mean average weight uranium per particle $(g)$ : \\
Uncertainty in mean average weight uranium per particle $(\mathrm{a})$ :
\end{tabular}} & \multicolumn{3}{|c|}{$\begin{array}{l}3.93 \mathrm{E}-04 \\
6.56 \mathrm{E}-07 \\
\end{array}$} \\
\hline & & First Leach & Second Leach & Total \\
\hline & Burn-leach solution ID: & $B 09062301$ & B09062401 & \\
\hline & Number of compacts: & & & \\
\hline & Total volume of leach solution $(\mathrm{ml})$ : & 50.0 & 50.5 & \\
\hline & Radiochemical laboratory analysis number: & $2014-031$ & $2014-036$ & \\
\hline & Measured uranium concentration $(\mu \mathrm{g} / \mathrm{ml}$ : $:$ : & $\frac{2014051}{3.49 \mathrm{E}-01}$ & $\frac{2014050}{4.71 E-03}$ & \\
\hline & Uncertainty in uranium concentration $(\mathrm{\mu g} / \mathrm{ml})$ : & $3.49 \mathrm{E}-02$ & $4.71 E-04$ & \\
\hline & Weight uranium leached $(g)$ : & $1.75 \mathrm{E}-05$ & $2.38 \mathrm{E}-07$ & $1.77 \mathrm{E}-05$ \\
\hline & Uncertainty in weight uranium leached $(9)$ : & $1.76 \mathrm{E}-06$ & $2.40 E-08$ & $1.76 \mathrm{E}-06$ \\
\hline & Number of leached kernels: & 0.0 & 0.0 & 0.0 \\
\hline & Uncertainty in number of leached kernels: & 0.0 & 0.0 & 0.0 \\
\hline \multirow{5}{*}{$\mathrm{Fe}$} & Measured concentration of impurity in sample $(\mathrm{\mu g} / \mathrm{ml})$ : & $7.13 \mathrm{E}-02$ & $<4.12 \mathrm{E}-02$ & \\
\hline & Uncorrected weight of impurity in sample $(\mu g)$ : & 3.57 & $<2.08$ & $<5.65$ \\
\hline & Weight of impurity in blank $(\mu \mathrm{g})$ : & $<2.18$ & $<2.16$ & \\
\hline & Minimum corrected weight of impurity in sample $(\mu \mathrm{g})$ : & 1.38 & 0.00 & 1.38 \\
\hline & Maximum corrected weight of impurity in sample $(\mu g)$ : & 3.57 & 2.08 & 5.65 \\
\hline \multirow{5}{*}{$\mathrm{Cr}$} & Measured concentration of impurity in sample $(\mathrm{\mu g} / \mathrm{ml})$ : & $1.23 \mathrm{E}-02$ & $<2.00 \mathrm{E}-03$ & $\mathrm{Cr}$ \\
\hline & Uncorrected weight of impurity in sample $(\mu \mathrm{g})$ : & 0.62 & $<0.10$ & $<0.72$ \\
\hline & Weight of impurity in blank $(\mu \mathrm{g})$ : & $<0.11$ & $<0.11$ & \\
\hline & Minimum corrected weight of impurity in sample $(\mu \mathrm{g})$ : & 0.51 & 0.00 & 0.51 \\
\hline & Maximum corrected weight of impurity in sample $(\mu \mathrm{g})$ : & 0.62 & 0.10 & 0.72 \\
\hline \multirow{5}{*}{ Mn } & Measured concentration of impurity in sample $(\mu \mathrm{g} / \mathrm{ml})$ : & $<1.91 \mathrm{E}-03$ & $<1.91 \mathrm{E}-03$ & $\mathrm{Mn}$ \\
\hline & Uncorrected weight of impurity in sample $(\mu \mathrm{g})$ : & $<0.10$ & $<0.10$ & $<0.19$ \\
\hline & Weight of impurity in blank $(\mu \mathrm{g})$ : & $<0.10$ & $<0.10$ & \\
\hline & Minimum corrected weight of impurity in sample $(\mu \mathrm{g})$ : & 0.00 & 0.00 & 0.00 \\
\hline & Maximum corrected weight of impurity in sample $(\mu g)$ : & 0.10 & 0.10 & 0.19 \\
\hline \multirow{5}{*}{ Co } & Measured concentration of impurity in sample $(\mu \mathrm{g} / \mathrm{ml})$ : & $<1.62 \mathrm{E}-03$ & $<1.62 \mathrm{E}-03$ & Co \\
\hline & Uncorrected weight of impurity in sample $(\mu \mathrm{g})$ : & $<0.08$ & $<0.08$ & $<0.16$ \\
\hline & Weight of impurity in blank $(\mu \mathrm{g})$ : & $<0.09$ & $<0.09$ & \\
\hline & Minimum corrected weight of impurity in sample $(\mu \mathrm{g})$ : & 0.00 & 0.00 & 0.00 \\
\hline & Maximum corrected weight of impurity in sample $(\mathrm{ug})$ : & 0.08 & 0.08 & 0.16 \\
\hline \multirow{5}{*}{$\mathrm{Ni}$} & Measured concentration of impurity in sample $(\mu \mathrm{g} / \mathrm{ml})$ : & $1.41 \mathrm{E}-02$ & $<8.00 \mathrm{E}-03$ & $\mathrm{Ni}$ \\
\hline & Uncorrected weight of impurity in sample $(\mu \mathrm{g})$ : & 0.71 & $<0.40$ & $<1.11$ \\
\hline & Weight of impurity in blank $(\mu g)$ : & $<0.42$ & $<0.42$ & \\
\hline & Minimum corrected weight of impurity in sample $(\mu \mathrm{g})$ : & 0.28 & 0.00 & 0.28 \\
\hline & Maximum corrected weight of impurity in sample $(\mu \mathrm{g})$ : & 0.71 & 0.40 & 1.11 \\
\hline \multirow{5}{*}{$\mathrm{Ca}$} & Measured concentration of impurity in sample $(\mu \mathrm{g} / \mathrm{ml})$ : & $2.36 \mathrm{E}+00$ & $3.14 \mathrm{E}-01$ & $\mathbf{C a}$ \\
\hline & Uncorrected weight of impurity in sample $(\mu \mathrm{g})$ : & 118.00 & 15.86 & 133.86 \\
\hline & Weight of impurity in blank $(\mu g)$ : & 24.12 & $<5.25$ & \\
\hline & Minimum corrected weight of impurity in sample $(\mu \mathrm{g})$ : & 93.89 & 10.61 & 104.49 \\
\hline & Maximum corrected weight of impurity in sample $(\mathrm{ug})$ : & 93.89 & 15.86 & 109.74 \\
\hline \multirow{5}{*}{ Al } & Measured concentration of impurity in sample $(\mu \mathrm{g} / \mathrm{ml})$ : & $1.24 \mathrm{E}+00$ & $4.56 \mathrm{E}-02$ & Al \\
\hline & Uncorrected weight of impurity in sample $(\mu \mathrm{g})$ : & 62.00 & 2.30 & 64.30 \\
\hline & Weight of impurity in blank $(\mu g)$ : & 3.59 & $<1.00$ & \\
\hline & Minimum corrected weight of impurity in sample $(\mu g)$ : & 58.41 & 1.30 & 59.71 \\
\hline & Maximum corrected weight of impurity in sample $(\mu g)$ : & 58.41 & 2.30 & 60.71 \\
\hline \multirow{5}{*}{$\mathrm{Ti}$} & Measured concentration of impurity in sample $(\mu \mathrm{g} / \mathrm{ml})$ : & $1.51 \mathrm{E}-01$ & $<8.00 \mathrm{E}-03$ & $\mathrm{Ti}$ \\
\hline & Uncorrected weight of impurity in sample $(\mathrm{\mu g})$ : & 7.55 & $<0.40$ & $<7.95$ \\
\hline & $\begin{array}{l}\text { Weight of impurity in blank }(\mu \mathrm{g}) \text { : } \\
\text { W }\end{array}$ & $<0.42$ & $<0.42$ & \\
\hline & Minimum corrected weight of impurity in sample $(\mathrm{\mu g})$ : & 7.13 & 0.00 & 7.13 \\
\hline & Maximum corrected weight of impurity in sample $(\mu \mathrm{g})$ : & 7.55 & 0.40 & 7.95 \\
\hline \multirow{5}{*}{$\mathbf{v}$} & Measured concentration of impurity in sample $(\mu \mathrm{g} / \mathrm{ml})$ : & $9.78 \mathrm{E}-01$ & $6.91 \mathrm{E}-03$ & \\
\hline & Uncorrected weight of impurity in sample $(\mu g)$ : & 48.90 & 0.35 & 49.25 \\
\hline & Weight of impurity in blank $(\mu \mathrm{g})$ : & $<0.11$ & $<0.11$ & \\
\hline & Minimum corrected weight of impurity in sample $(\mu g)$ : & 48.79 & 0.24 & 49.04 \\
\hline & Maximum corrected weight of impurity in sample $(\mu g)$ : & 48.90 & 0.35 & 49.25 \\
\hline \multicolumn{5}{|c|}{ Comments } \\
\hline \multicolumn{5}{|c|}{ Checked against official results of analyses for RMAL2014 by FCM on 7/23/2009 } \\
\hline
\end{tabular}

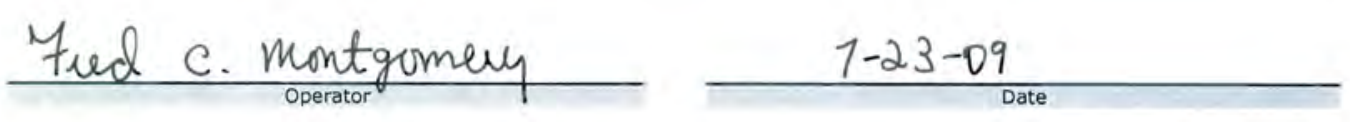


Data Report Form DRF-26B: Measurement of SIC Burn-Leach Defects or Impurities by Burn-Leach

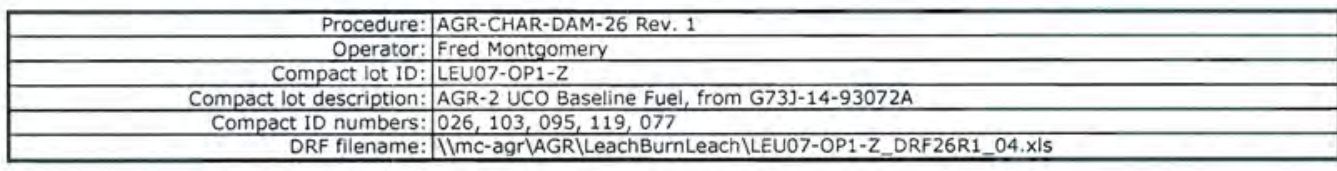

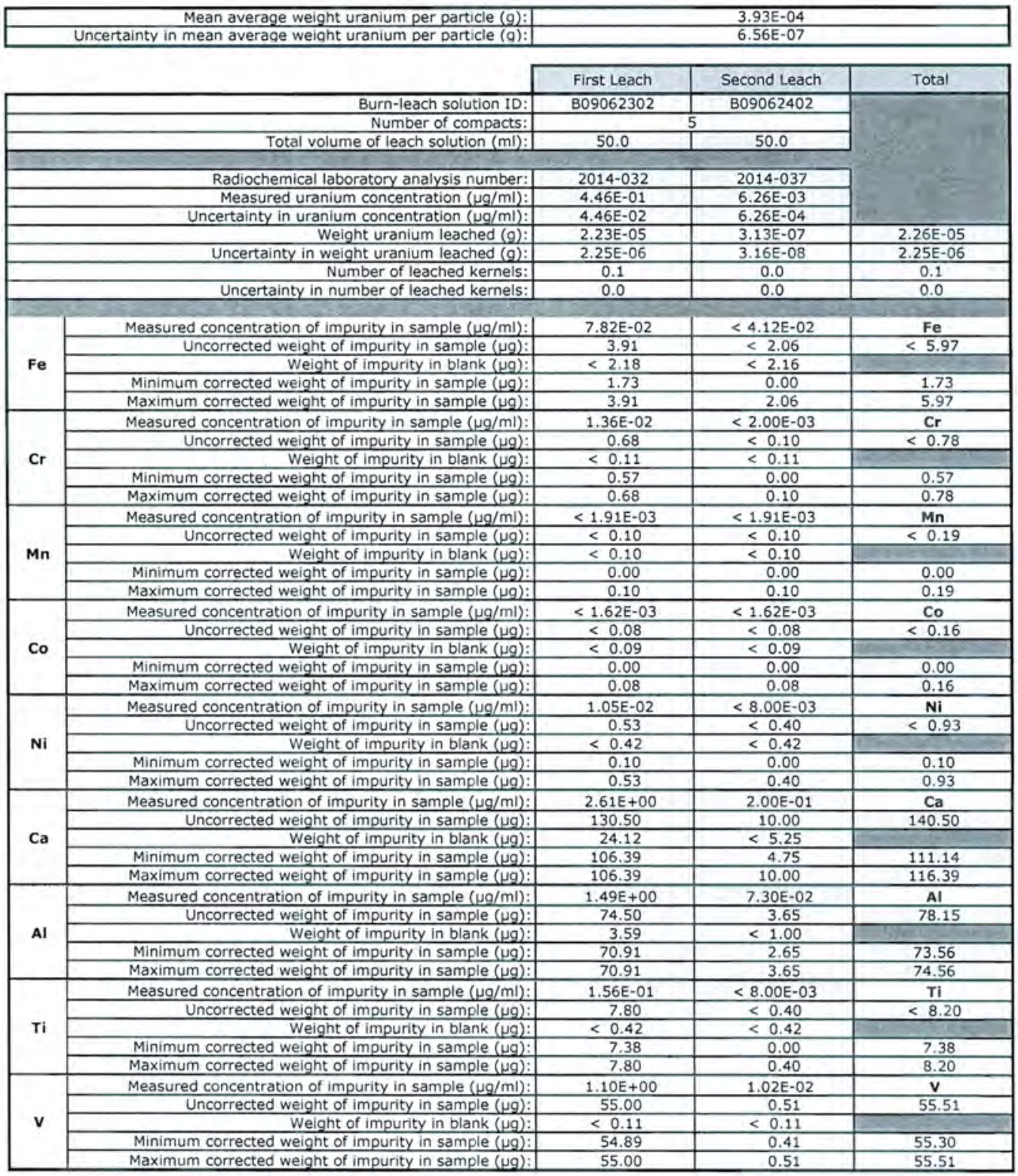

comments

Checked against official results of analyses for RMAL2014 by FCM on 7/23/2009

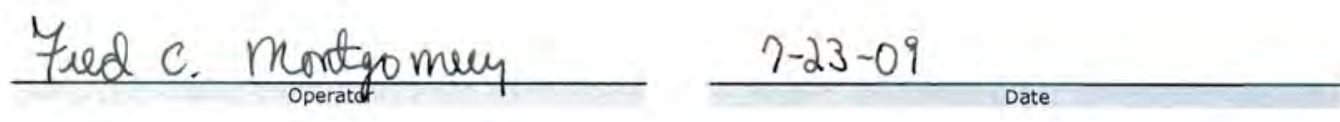


Data Report Form DRF-26B: Measurement of SIC Burn-Leach Defects or Impuritles by Burn-Leach

\begin{tabular}{|c|c|}
\hline $\begin{array}{c}\text { Procedure: } \\
\text { Operator: } \\
\end{array}$ & $\begin{array}{l}\text { AGR-CHAR-DAM-26 Rev. } 1 \\
\text { Fred Montgomery }\end{array}$ \\
\hline Compact lot ID: & LEU07-OP1-Z \\
\hline Compact lot description: & AGR-2 UCO Baseline Fuel, fr \\
\hline Compact ID numbers: & $112,009,155,056,001$ \\
\hline
\end{tabular}

\begin{tabular}{|c|c|c|c|c|}
\hline \multicolumn{2}{|r|}{$\begin{array}{r}\text { Mean average weight uranium per particle }(g): \\
\text { Uncertainty in mean average weight uranium per particle }(g):\end{array}$} & \multicolumn{3}{|c|}{$\begin{array}{l}3.93 E-04 \\
6.56 E-07\end{array}$} \\
\hline & & First Leach & Second Leach & Total \\
\hline & Burn-leach solution ID: & $B 09062303$ & 809062403 & \\
\hline & Number of compacts: & & & \\
\hline & Total volume of leach solution $(\mathrm{ml})$ : & 51.5 & 51.0 & \\
\hline & & & & \\
\hline & & $2014-033$ & $2014-038$ & \\
\hline & $\begin{array}{l}\text { Measured uranium concentration }(\mu \mathrm{g} / \mathrm{ml}) \text { : } \\
\text { Uncertainty in uranium concentration }(\mu \mathrm{g} / \mathrm{ml}) \text { : }\end{array}$ & $\frac{3.19 \mathrm{E}-01}{3.19 \mathrm{E}-02}$ & $\frac{9.31 \mathrm{E}-03}{9.31 \mathrm{E}-0.4}$ & \\
\hline & $\begin{array}{r}\text { Weight uranium leached }(9): \\
\end{array}$ & $1.64 \mathrm{E}-05$ & $4.75 E-07$ & $1.69 \mathrm{E}-05$ \\
\hline & Uncertainty in weight uranium leached $(9)$ : & $1.66 \mathrm{E}-06$ & $4.79 \mathrm{E}-08$ & $1.66 \mathrm{E}-06$ \\
\hline & Number of leached kernels: & 0.0 & 0.0 & 0.0 \\
\hline & Uncertainty in number of leached kernels: & 0.0 & 0.0 & 0.0 \\
\hline \multirow{5}{*}{$\mathrm{Fe}$} & Measured concentration of impurity in sample $(\mu \mathrm{g} / \mathrm{ml})$ : & $8.79 \mathrm{E}-02$ & $<4.12 \mathrm{E}-02$ & $\mathrm{Fe}$ \\
\hline & Uncorrected weight of impurity in sample $(\mu g)$ : & 4.53 & $<2.10$ & $<6.63$ \\
\hline & Weight of impurity in blank $(\mu g)$ : & $<2.18$ & $<2.16$ & \\
\hline & Minimum corrected weight of impurity in sample $(\mu g)$ : & 2.34 & 0.00 & 2.34 \\
\hline & Maximum corrected weight of impurity in sample $(\mu g)$ : & 4.53 & 2.10 & 6.63 \\
\hline \multirow{5}{*}{$\mathrm{Cr}$} & Measured concentration of impurity in sample $(\mu \mathrm{g} / \mathrm{ml})$ : & $1.48 \mathrm{E}-02$ & $<2.00 E-03$ & $\mathrm{Cr}$ \\
\hline & Uncorrected weight of impurity in sample $(\mu \mathrm{g})$ : & 0.76 & $<0.10$ & $<0.86$ \\
\hline & Weight of impurity in blank $(\mu \mathrm{g})$ : & $<0.11$ & $<0.11$ & \\
\hline & Minimum corrected weight of impurity in sample $(\mu \mathrm{g})$ : & 0.66 & 0.00 & 0.66 \\
\hline & Maximum corrected weight of impurity in sample $(\mu \mathrm{g})$ : & 0.76 & 0.10 & 0.86 \\
\hline \multirow{5}{*}{ Mn } & Measured concentration of impurity in sample $(\mu \mathrm{g} / \mathrm{ml})$ : & $<1.91 \mathrm{E}-03$ & $<1.91 \mathrm{E}-03$ & $\mathrm{Mn}$ \\
\hline & Uncorrected weight of impurity in sample $(\mu \mathrm{g})$ : & $<0.10$ & $<0.10$ & $<0.20$ \\
\hline & Weight of impurity in blank $(\mu g)$ : & $<0.10$ & $<0.10$ & \\
\hline & Minimum corrected weight of impurity in sample $(\mu \mathrm{g})$ : & 0.00 & 0.00 & 0.00 \\
\hline & Maximum corrected weight of impurity in sample $(\mu \mathrm{g})$ : & 0.10 & 0.10 & 0.20 \\
\hline \multirow{5}{*}{ co } & Measured concentration of impurity in sample $(\mu \mathrm{g} / \mathrm{ml})$ : & $<1.62 \mathrm{E}-03$ & $<1.62 \mathrm{E}-03$ & \\
\hline & Uncorrected weight of impurity in sample $(\mu \mathrm{g})$ : & $<0.08$ & $<0.08$ & $<0.17$ \\
\hline & Weight of impurity in blank $(\mu \mathrm{g})$ : & $<0.09$ & $<0.09$ & \\
\hline & Minimum corrected weight of impurity in sample $(\mu g)$ : & 0.00 & 0.00 & 0.00 \\
\hline & Maximum corrected weight of impurity in sample $(\mu g)$ : & 0.08 & 0.08 & 0.17 \\
\hline \multirow{5}{*}{$\mathrm{Ni}$} & Measured concentration of impurity in sample $(\mathrm{\mu g} / \mathrm{ml})$ : & $9.56 \mathrm{E}-03$ & $<8.00 \mathrm{E}-03$ & $\mathrm{Ni}$ \\
\hline & Uncorrected weight of impurity in sample $(\mu \mathrm{g})$ : & 0.49 & $<0.41$ & $<0.90$ \\
\hline & Weight of impurity in blank $(\mu \mathrm{g})$ : & $<0.42$ & $<0.42$ & \\
\hline & Minimum corrected weight of impurity in sample $(\mu g)$ : & 0.07 & 0.00 & 0.07 \\
\hline & Maximum corrected weight of impurity in sample $(\mu \mathrm{g})$ : & 0.49 & 0.41 & 0.90 \\
\hline \multirow{5}{*}{ Ca } & Measured concentration of impurity in sample $(\mu \mathrm{g} / \mathrm{ml})$ : & $1.83 E+00$ & $4.04 \mathrm{E}-01$ & $\mathrm{Ca}$ \\
\hline & Uncorrected weight of impurity in sample $(\mu \mathrm{g})$ : & 94.25 & 20.60 & 114.85 \\
\hline & Weight of impurity in blank $(\mu \mathrm{g})$ : & 24.12 & $<5.25$ & \\
\hline & Minimum corrected weight of impurity in sample $(\mu \mathrm{g})$ : & 70.13 & 15.35 & 85.48 \\
\hline & Maximum corrected weight of impurity in sample $(\mu \mathrm{g})$ : & 70.13 & 20.60 & 90.73 \\
\hline \multirow{5}{*}{ Al } & Measured concentration of impurity in sample $(\mu \mathrm{g} / \mathrm{ml})$ : & $1.23 E+00$ & $5.92 \mathrm{E}-02$ & Al \\
\hline & Uncorrected weight of impurity in sample $(\mu g)$ : & 63.35 & 3.02 & 66.36 \\
\hline & Weight of impurity in blank $(\mu \mathrm{g})$ : & 3.59 & $<1.00$ & \\
\hline & Minimum corrected weight of impurity in sample $(\mu \mathrm{\mu g})$ : & 59.76 & 2.02 & 61.77 \\
\hline & Maximum corrected weight of impurity in sample $(\mu q)$ : & 59.76 & 3.02 & 62.78 \\
\hline \multirow{5}{*}{$\mathrm{Ti}$} & Measured concentration of impurity in sample $(\mu \mathrm{g} / \mathrm{ml})$ : & $1.68 \mathrm{E}-01$ & $<8.00 E-03$ & $\mathrm{Ti}$ \\
\hline & Uncorrected weight of impurity in sample $(\mu \mathrm{g})$ : & 8.65 & $<0.41$ & $<9.06$ \\
\hline & Weight of impurity in blank $(\mu \mathrm{g})$ : & $<0.42$ & $<0.42$ & \\
\hline & Minimum corrected weight of impurity in sample $(\mu \mathrm{g})$ : & 8.23 & 0.00 & 8.23 \\
\hline & Maximum corrected weight of impurity in sample $(\mu \mathrm{g})$ : & 8.65 & 0.41 & 9.06 \\
\hline \multirow{5}{*}{ v } & Measured concentration of impurity in sample $(\mu \mathrm{g} / \mathrm{ml})$ : & $1.01 E+00$ & $1.01 \mathrm{E}-02$ & $\mathrm{v}$ \\
\hline & Uncorrected weight of impurity in sample $(\mu \mathrm{g})$ : & 52.02 & 0.52 & 52.53 \\
\hline & Weight of impurity in blank $(\mu \mathrm{g})$ : & $<0.11$ & $<0.11$ & \\
\hline & Minimum corrected weight of impurity in sample $(\mu \mathrm{g})$ : & $\frac{51.91}{52.02}$ & $\frac{0.41}{0.52}$ & $\frac{52.32}{52.53}$ \\
\hline & Maximum corrected weight of impurity in sample $(\mu g)$ : & 52.02 & & 52.53 \\
\hline
\end{tabular}

comments

Checked against official results of analyses for RMAL2014 by FCM on 7/23/2009

$\frac{\text { Fied c. Montgomeery }}{\text { opetator }}$

$1-23-09$ 
Data Report Form DRF-26B: Measurement of SiC Burn-Leach Defects or Impurities by Burn-Leach

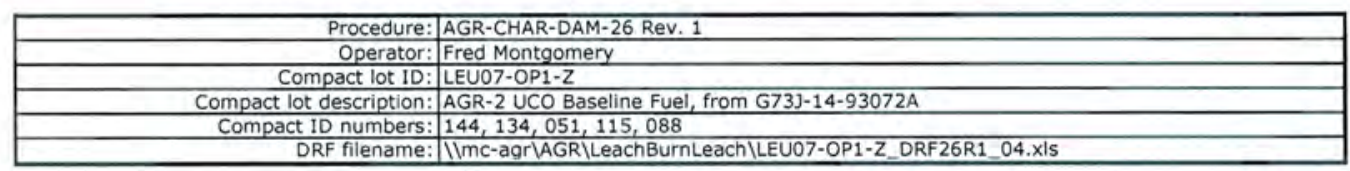

\begin{tabular}{|c|c|c|c|c|}
\hline \multicolumn{2}{|r|}{$\begin{array}{c}\text { Mean average weight uranium per particle }(g) \text { : } \\
\text { Uncertainty in mean average weight uranium per particle }(q):\end{array}$} & \multicolumn{3}{|c|}{$\frac{3.93 \mathrm{E}-04}{6.56 \mathrm{E}-07}$} \\
\hline & & First Leach & Second Leach & Total \\
\hline & Burn-leach solution ID: & 809062304 & $B 09062404$ & \\
\hline & Number of compacts: & & & \\
\hline & Total volume of leach solution $(\mathrm{ml})$ : & 49.5 & 50.5 & \\
\hline & & & & \\
\hline & Radiochemical laboratory analysis number: & $2014-034$ & 2014-039 & \\
\hline & Measured uranium concentration $(\mu \mathrm{g} / \mathrm{ml})$ : & $3.38 \mathrm{E}-01$ & $9.45 \mathrm{E}-03$ & \\
\hline & Uncertainty in uranium concentration $(\mathrm{\mu g} / \mathrm{ml})$ : & $3.38 \mathrm{E}-02$ & $9.45 E-04$ & \\
\hline & Weight uranium leached $(\mathrm{g})$ : & $1.67 \mathrm{E}-05$ & $4.77 \mathrm{E}-07$ & $1.72 \mathrm{E}-05$ \\
\hline & Uncertainty in weight uranium leached $(\mathrm{g})$ : & $1.69 \mathrm{E}-06$ & $4.81 \mathrm{E}-08$ & $1.69 \mathrm{E}-06$ \\
\hline & Number of leached kernels: & 0.0 & 0.0 & 0.0 \\
\hline & Uncertainty in number of leached kernels: & 0.0 & 0.0 & 0.0 \\
\hline \multirow{5}{*}{$\mathrm{Fe}$} & Measured concentration of impurity in sample $(\mu \mathrm{g} / \mathrm{ml})$ : & $7.43 E-02$ & $<4.12 \mathrm{E}-02$ & $\mathrm{Fe}$ \\
\hline & Uncorrected weight of impurity in sample $(\mu \mathrm{g})$ : & 3.68 & $<2.08$ & $<5.76$ \\
\hline & Weight of impurity in blank $(\mu g)$ : & $<2.18$ & $<2.16$ & \\
\hline & Minimum corrected weight of impurity in sample $(\mu g)$ : & 1.49 & 0.00 & 1.49 \\
\hline & Maximum corrected weight of impurity in sample $(\mu g)$ : & 3.68 & 2.08 & 5.76 \\
\hline \multirow{5}{*}{$\mathrm{cr}$} & Measured concentration of impurity in sample $(\mu \mathrm{g} / \mathrm{ml})$ : & $1.20 \mathrm{E}-02$ & $<2.00 \mathrm{E}-03$ & $\mathrm{Cr}$ \\
\hline & Uncorrected weight of impurity in sample $(\mu g)$ : & 0.59 & $<0.10$ & $<0.70$ \\
\hline & Weight of impurity in blank $(\mathrm{\mu g})$ : & $<0.11$ & $<0.11$ & \\
\hline & Minimum corrected weight of impurity in sample $(\mu \mathrm{g})$ : & 0.49 & 0.00 & 0.49 \\
\hline & Maximum corrected weight of impurity in sample $(\mu \mathrm{g})$ : & 0.59 & 0.10 & 0.70 \\
\hline \multirow{5}{*}{ Mn } & Measured concentration of impurity in sample $(\mu \mathrm{g} / \mathrm{ml})$ : & $<1.91 \mathrm{E}-03$ & $<1.91 \mathrm{E}-03$ & Mn \\
\hline & Uncorrected weight of impurity in sample $(\mu \mathrm{g})$ : & $<0.09$ & $<0.10$ & $<0.19$ \\
\hline & Weight of impurity in blank $(\mu g)$ : & $<0.10$ & $<0.10$ & \\
\hline & Minimum corrected weight of impurity in sample $(\mathrm{\mu g})$ : & 0.00 & 0.00 & 0.00 \\
\hline & Maximum corrected weight of impurity in sample $(\mu g)$ : & 0.09 & 0.10 & 0.19 \\
\hline \multirow{5}{*}{ co } & Measured concentration of impurity in sample $(\mu \mathrm{g} / \mathrm{ml})$ : & $<1.62 \mathrm{E}-03$ & $<1.62 \mathrm{E}-03$ & Co \\
\hline & Uncorrected weight of impurity in sample $(\mu \mathrm{g})$ : & $<0.08$ & $<0.08$ & $<0.16$ \\
\hline & Weight of impurity in blank $(\mu g)$ : & $<0.09$ & $<0.09$ & \\
\hline & Minimum corrected weight of impurity in sample $(\mathrm{\mu g})$ : & 0.00 & 0.00 & 0.00 \\
\hline & Maximum corrected weight of impurity in sample $(\mu \mathrm{g})$ : & 0.08 & 0.08 & 0.16 \\
\hline \multirow{5}{*}{$\mathrm{Ni}$} & Measured concentration of impurity in sample $(\mu \mathrm{g} / \mathrm{ml})$ : & $8.93 \mathrm{E}-03$ & $<8.00 \mathrm{E}-03$ & $\mathrm{Ni}$ \\
\hline & Uncorrected weight of impurity in sample $(\mu \mathrm{g})$ : & 0.44 & $<0.40$ & $<0.85$ \\
\hline & Weight of impurity in blank $(\mu g)$ : & $<0.42$ & $<0.42$ & \\
\hline & Minimum corrected weight of impurity in sample $(\mathrm{\mu g})$ : & 0.02 & 0.00 & 0.02 \\
\hline & Maximum corrected weight of impurity in sample $(\mu g)$ : & 0.44 & 0.40 & 0.85 \\
\hline \multirow{5}{*}{$\mathbf{C a}$} & Measured concentration of impurity in sample $(\mu \mathrm{g} / \mathrm{ml})$ : & $2.38 E+00$ & $4.38 \mathrm{E}-01$ & $\mathbf{C a}$ \\
\hline & Uncorrected weight of impurity in sample $(\mu q)$ : & 117.81 & 22.12 & 139.93 \\
\hline & Weight of impurity in blank $(\mu \mathrm{g})$ : & 24.12 & $<5.25$ & \\
\hline & Minimum corrected weight of impurity in sample $(\mu q)$ : & 93.70 & 16.87 & 110.56 \\
\hline & Maximum corrected weight of impurity in sample $(\mu g)$ : & 93.70 & 22.12 & 115.81 \\
\hline \multirow{5}{*}{ Al } & Measured concentration of impurity in sample $(\mu \mathrm{g} / \mathrm{ml})$ : & $1.15 E+00$ & $7.26 \mathrm{E}-02$ & Al \\
\hline & Uncorrected weight of impurity in sample $(\mu g)$ : & 56.93 & 3.67 & 60.59 \\
\hline & Weight of impurity in blank $(\mu g)$ : & 3.59 & $<1.00$ & \\
\hline & Minimum corrected weight of impurity in sample $(\mu g)$ : & 53.34 & 2.66 & 56.00 \\
\hline & Maximum corrected welght of impurity in sample $(\mu g)$ : & 53.34 & 3.67 & 57.00 \\
\hline \multirow{5}{*}{$\mathrm{Ti}$} & Measured concentration of impurity in sample $(\mu \mathrm{g} / \mathrm{ml})$ : & $1.58 \mathrm{E}-01$ & $<8.00 \mathrm{E}-03$ & $\mathrm{Ti}$ \\
\hline & Uncorrected weight of impurity in sample $(\mu g)$ : & 7.82 & $<0.40$ & $<8.23$ \\
\hline & Weight of impurity in blank $(\mu \mathrm{g})$ : & $<0.42$ & $<0.42$ & \\
\hline & Minimum corrected weight of impurity in sample $(\mu \mathrm{g})$ : & 7.40 & 0.00 & 7.40 \\
\hline & Maximum corrected weight of impurity in sample $(\mu g)$ : & 7.82 & 0.40 & 8.23 \\
\hline \multirow{5}{*}{ v } & Measured concentration of impurity in sample $(\mu \mathrm{g} / \mathrm{ml})$ : & $8.74 \mathrm{E}-01$ & $1.05 \mathrm{E}-02$ & $\mathbf{v}$ \\
\hline & Uncorrected weight of impurity in sample $(\mu g)$ : & 43.26 & 0.53 & 43.79 \\
\hline & Weight of impurity in blank $(\mu \mathrm{gg}):$ & $<0.11$ & $<0.11$ & \\
\hline & Minimum corrected weight of impurity in sample $(\mu \mathrm{g})$ : & 43.16 & 0.43 & 43.58 \\
\hline & Maximum corrected weight of impurity in sample $(\mu q)$ : & & & \\
\hline
\end{tabular}

Comments

Checked against official results of analyses for RMAL2014 by FCM on 7/23/2009

Fied c. Montgomey $\frac{7-23-09}{\text { operator }}$ 
Data Report Form DRF-26B: Measurement of SiC Burn-Leach Defects or Impurities by Burn-Leach

\begin{tabular}{|r|l|}
\hline Procedure: & AGR-CHAR-DAM-26 Rev. 1 \\
\hline Operator: & Fred Montgomery \\
\hline Compact lot ID: & LEU07-OP1-Z \\
\hline Compact lot description: & AGR-2 UCO Baseline Fuel, from G73J-14-93072A \\
\hline Compact ID numbers: & Burn-Leach Blank \\
\hline DRF filename: & $\backslash$ IImC-agr\AGR\LeachBurnLeach \LEU07-OP1-Z_DRF26R1_04.xIS \\
\hline
\end{tabular}

\begin{tabular}{|c|c|c|c|c|}
\hline \multirow{2}{*}{\multicolumn{2}{|c|}{$\begin{array}{l}\text { Mean average weight uranium per particle }(\mathrm{g}) \text { : } \\
\text { Uncertainty in mean average weight uranium per particle }(\mathrm{g}) \text { : }\end{array}$}} & \multicolumn{3}{|c|}{$3.93 E-04$} \\
\hline & & \multicolumn{3}{|c|}{$6.56 \mathrm{E}-07$} \\
\hline & & First Leach & Second Leach & Total \\
\hline & Burn-leach solution ID: & B09062305 & B09062405 & \\
\hline & Number of compacts: & \multicolumn{2}{|c|}{ None } & \\
\hline & Total volume of leach solution $(\mathrm{ml})$ : & 53.0 & 52.5 & \\
\hline & 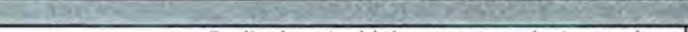 & 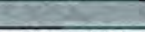 & 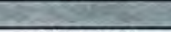 & \\
\hline & Radiochemical laboratory analysis number: & 2014-035 & $2014-040$ & \\
\hline & Measured uranium concentration $(\mu \mathrm{g} / \mathrm{ml})$ : & $<5.00 \mathrm{E}-04$ & $<5.00 \mathrm{E}-04$ & \\
\hline \multicolumn{5}{|c|}{ Uncertainty in uranium concentration $(\mu \mathrm{g} / \mathrm{ml})$ : } \\
\hline & Weight uranium leached $(g)$ : & $<2.65 E-08$ & $<2.63 \mathrm{E}-08$ & $<5.28 \mathrm{E}-08$ \\
\hline \multicolumn{5}{|c|}{ Uncertainty in weight uranium leached $(\mathrm{g})$ : } \\
\hline & Number of leached kernels: & 0.0 & 0.0 & 0.0 \\
\hline \multicolumn{5}{|c|}{ Uncertainty in number of leached kernels: } \\
\hline \multirow{2}{*}{$\mathrm{Fe}$} & Measured concentration $(\mu \mathrm{g} / \mathrm{ml})$ : & $<4.12 \mathrm{E}-02$ & $<4.12 \mathrm{E}-02$ & $\mathrm{Fe}$ \\
\hline & Total weight of leached impurity $(\mu \mathrm{g})$ : & $<2.18$ & $<2.16$ & $<4.35$ \\
\hline \multirow{2}{*}{$\mathrm{Cr}$} & Measured concentration $(\mu \mathrm{g} / \mathrm{ml})$ : & $<2.00 \mathrm{E}-03$ & $<2.00 \mathrm{E}-03$ & $\mathrm{Cr}$ \\
\hline & Total weight of leached impurity $(\mu \mathrm{g})$ : & $<0.11$ & $<0.11$ & $<0.21$ \\
\hline \multirow{2}{*}{ Mn } & Measured concentration $(\mu \mathrm{g} / \mathrm{ml})$ : & $<1.91 \mathrm{E}-03$ & $<1.91 \mathrm{E}-03$ & Mn \\
\hline & Total weight of leached impurity $(\mu \mathrm{g})$ : & $<0.10$ & $<0.10$ & $<0.20$ \\
\hline \multirow{2}{*}{ Co } & Measured concentration $(\mu \mathrm{g} / \mathrm{ml})$ : & $<1.62 \mathrm{E}-03$ & $<1.62 \mathrm{E}-03$ & Co \\
\hline & Total weight of leached impurity $(\mu \mathrm{g})$ : & $<0.09$ & $<0.09$ & $<0.17$ \\
\hline \multirow{2}{*}{$\mathrm{Ni}$} & Measured concentration $(\mu \mathrm{g} / \mathrm{ml})$ : & $<8.00 \mathrm{E}-03$ & $<8.00 \mathrm{E}-03$ & $\mathrm{Ni}$ \\
\hline & Total weight of leached impurity $(\mu \mathrm{g})$ : & $<0.42$ & $\leq 0.42$ & $<0.84$ \\
\hline \multirow{2}{*}{$\mathrm{Ca}$} & Measured concentration $(\mu \mathrm{g} / \mathrm{ml})$ : & 4.55E-01 & $<1.00 \mathrm{E}-01$ & $\mathrm{Ca}$ \\
\hline & Total weight of leached impurity $(\mu \mathrm{g})$ : & 24.12 & $<5.25$ & $<29.37$ \\
\hline \multirow{2}{*}{ Al } & Measured concentration $(\mu \mathrm{g} / \mathrm{ml})$ : & $6.77 \mathrm{E}-02$ & $<1.91 \mathrm{E}-02$ & $\mathbf{A l}$ \\
\hline & Total weight of leached impurity $(\mu \mathrm{g})$ : & 3.59 & $<1.00$ & $<4.59$ \\
\hline \multirow{2}{*}{$\mathrm{Ti}$} & Measured concentration $(\mu \mathrm{g} / \mathrm{ml})$ : & $<8.00 \mathrm{E}-03$ & $<8.00 \mathrm{E}-03$ & $\mathrm{Ti}$ \\
\hline & Total weight of leached impurity $(\mu \mathrm{g})$ : & $<0.42$ & $<0.42$ & $<0.84$ \\
\hline \multirow{2}{*}{ v } & Measured concentration $(\mu \mathrm{g} / \mathrm{ml})$ : & $<2.00 \mathrm{E}-03$ & $<2.00 \mathrm{E}-03$ & $\mathbf{V}$ \\
\hline & Total weight of leached impurity $(\mu \mathrm{g})$ : & $<0.11$ & $<0.11$ & $<0.21$ \\
\hline
\end{tabular}

Comments

Checked against official results of analyses for RMAL2014 by FCM on 7/23/2009

Fred C. Montgomeey
$7-23-09$

Date 
Data Report Form DRF-26A: Measurement of U Contamination or Impuritles by Deconsolidation Leach

\begin{tabular}{|c|c|}
\hline Procedure: A & AGR-CHAR-DAM-26 Rev. 1 \\
\hline $\begin{aligned} \text { Operator: } F \\
\text { Compact lot ID:L }\end{aligned}$ & Fred Montgomery \\
\hline Compact lot ID: L & LEU07-OP1-Z \\
\hline Compact lot description: $\mathrm{A}$ & AGR-2 UCO Baseline Fuel, from G73J-14-93072A \\
\hline Compact ID numbers: 1 & 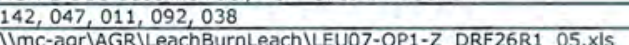 \\
\hline
\end{tabular}

\begin{tabular}{|c|c|c|c|c|}
\hline & $\begin{array}{l}\text { Mean average weight uranium per particle }(g) \text { : } \\
\text { Uncertainty in mean average weight uranium per particle }(\mathrm{g}) \text { : }\end{array}$ & & $\begin{array}{l}3.93 E-04 \\
6.56 E-07\end{array}$ & \\
\hline & & First Leach & Second Leach & Total \\
\hline & Deconsolidation-leach solution ID: & L09061801 & L09062201 & \\
\hline & Number of compacts: & & & \\
\hline & Total volume of leach solution $(\mathrm{ml})$ : & 155.0 & 126.0 & \\
\hline & Radiochemical laboratory analysis number: & $2014-016$ & $2014-021$ & \\
\hline & Measured uranium concentration $(\mathrm{\mu g} / \mathrm{ml})$ : & $3.47 \mathrm{E}-02$ & $1.03 E-02$ & \\
\hline & Uncertainty in uranium concentration $(\mu \mathrm{g} / \mathrm{ml})$ : & $3.47 \mathrm{E}-03$ & $1.03 E-03$ & \\
\hline & Weight uranium leached $(g)$ : & $5.38 \mathrm{E}-06$ & $1.30 \mathrm{E}-06$ & $6.68 \mathrm{E}-06$ \\
\hline & Uncertainty in weight uranium leached $(g)$ : & $5.38 \mathrm{E}-07$ & $1.30 \mathrm{E}-07$ & $5.54 \mathrm{E}-07$ \\
\hline & Effective number of exposed kernels: & 0.0 & 0.0 & 0.0 \\
\hline & Uncertainty in effective number of exposed kernels: & 0.0 & 0.0 & 0.0 \\
\hline & Measured concentration of impurity in sample $(\mathrm{ug} / \mathrm{ml})$ & $<4.12 \mathrm{E}-02$ & $<4,12 \mathrm{E}-02$ & $\mathrm{Fe}$ \\
\hline & Uncorrected weight of impurity in sample $(\mu \mathrm{g})$ & $<6.39$ & $<5.19$ & $<11.58$ \\
\hline $\mathrm{Fe}$ & Weight of impurity in blank $(\mu \mathrm{g})$ : & $<7.37$ & $<5.27$ & \\
\hline & Minimum corrected weight of impurity in sample $(\mu g)$ : & 0.00 & 0.00 & 0.00 \\
\hline & Maximum corrected weight of impurity in sample $(\mu g)$ : & 6.39 & 5.19 & 11.58 \\
\hline & Measured concentration of impurity in sample $(\mathrm{\mu g} / \mathrm{ml})$ : & $5.98 \mathrm{E}-03$ & $4.77 \mathrm{E}-03$ & $\mathrm{Cr}$ \\
\hline & Uncorrected weight of impurity in sample $(\mu g)$ : & 0.93 & 0.60 & 1.53 \\
\hline $\mathrm{Cr}$ & Weight of impurity in blank $(\mu \mathrm{g})$ : & $<0.36$ & $<0.26$ & \\
\hline & Minimum corrected weight of impurity in sample $(\mu g)$ : & 0.57 & 0.35 & 0.91 \\
\hline & Maximum corrected weight of impurity in sample $(\mu g)$ : & 0.93 & 0.60 & 1.53 \\
\hline & Measured concentration of impurity in sample $(\mathrm{\mu g} / \mathrm{ml})$ : & $<1.91 \mathrm{E}-03$ & $<1.91 \mathrm{E}-03$ & $\mathrm{Mn}$ \\
\hline & Uncorrected weight of impurity in sample $(\mu \mathrm{g})$ : & $<0.30$ & $<0.24$ & $<0.54$ \\
\hline $\mathrm{Mn}$ & Weight of impurity in blank $(\mu \mathrm{g})$ : & $<0.34$ & $<0.24$ & \\
\hline & Minimum corrected weight of impurity in sample $(\mu g)$ : & 0.00 & 0.00 & 0.00 \\
\hline & Maximum corrected weight of impurity in sample $(\mu g)$ : & 0.30 & 0.24 & 0.54 \\
\hline & Measured concentration of impurity in sample $(\mu \mathrm{g} / \mathrm{ml})$ : & $<1.62 \mathrm{E}-03$ & $<1.62 \mathrm{E}-03$ & Co \\
\hline & Uncorrected weight of impurity in sample $(\mu g)$ : & $<0.25$ & $<0.20$ & $<0.46$ \\
\hline Co & Weight of impurity in blank $(\mu \mathrm{g})$ : & $<0.29$ & $<0.21$ & \\
\hline & Minimum corrected weight of impurity in sample $(\mu \mathrm{g})$ : & 0.00 & 0.00 & 0.00 \\
\hline & Maximum corrected weight of impurity in sample $(\mu g)$ : & 0.25 & 0.20 & 0.46 \\
\hline & Measured concentration of impurity in sample $(\mu \mathrm{g} / \mathrm{ml})$ : & $<8.00 \mathrm{E}-03$ & $<8.00 \mathrm{E}-03$ & $\mathrm{Ni}$ \\
\hline & Uncorrected weight of impurity in sample $(\mu \mathrm{g})$ : & $<1.24$ & $<1.01$ & $<2.25$ \\
\hline $\mathrm{Ni}$ & Weight of impurity in blank $(\mu \mathrm{g})$ : & $<1.43$ & $<1.02$ & \\
\hline & Minimum corrected weight of impurity in sample $(\mu g)$ : & 0.00 & 0.00 & 0.00 \\
\hline & Maximum corrected weight of impurity in sample $(\mu g)$ : & 1.24 & 1.01 & 2.25 \\
\hline & Measured concentration of impurity in sample $(\mathrm{\mu g} / \mathrm{ml})$ : & $4.12 \mathrm{E}-01$ & $<1.00 \mathrm{E}-01$ & $\mathrm{Ca}$ \\
\hline & Uncorrected weight of impurity in sample $(\mu \mathrm{g})$ : & 63.86 & $<12.60$ & $<76.46$ \\
\hline $\mathrm{Ca}$ & Weight of impurity in blank $(\mu \mathrm{g})$ : & 137.11 & $<12.80$ & \\
\hline & Minimum corrected weight of impurity in sample $(\mu g)$ : & 0.00 & 0.00 & 0.00 \\
\hline & Maximum corrected weight of impurity in sample $(\mu g)$ : & 0.00 & 12.60 & 12.60 \\
\hline & Measured concentration of impurity in sample $(\mu \mathrm{g} / \mathrm{ml})$ : & $3.60 \mathrm{E}-01$ & $1.07 E-01$ & Al \\
\hline & Uncorrected weight of impurity in sample $(\mu \mathrm{g})$ : & 55.80 & 13.48 & 69.28 \\
\hline Al & Weight of impurity in blank $(\mu g)$ : & $<1.43$ & $<1.02$ & 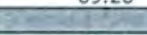 \\
\hline & Minimum corrected weight of impurity in sample $(\mu g)$ : & 54.37 & 12.46 & 66.83 \\
\hline & Maximum corrected weight of impurity in sample $(\mu g)$ : & 55.80 & 13.48 & 69.28 \\
\hline & Measured concentration of impurity in sample $(\mu \mathrm{g} / \mathrm{ml})$ : & $3.21 \mathrm{E}-02$ & $2.31 E-02$ & $\mathrm{Ti}$ \\
\hline & Uncorrected weight of impurity in sample $(\mu g)$ : & 4.98 & 2.91 & 7.89 \\
\hline $\mathbf{T i}$ & Weight of impurity in blank $(\mu \mathrm{g})$ : & $<1.43$ & $<1.02$ & \\
\hline & Minimum corrected weight of impurity in sample $(\mu g)$ : & 3.54 & 1.89 & 5.43 \\
\hline & Maximum corrected weight of impurity in sample $(\mu \mathrm{g})$ : & 4.98 & 2.91 & 7.89 \\
\hline & Measured concentration of impurity in sample $(\mu \mathrm{g} / \mathrm{ml})$ : & $2.07 E-01$ & $4.88 \mathrm{E}-02$ & $\mathrm{v}$ \\
\hline & Uncorrected weight of impurity in sample $(\mu \mathrm{g})$ : & 32.09 & 6.15 & 38.23 \\
\hline v & Weight of impurity in blank $(\mu g)$ : & $<0.36$ & $<0.26$ & \\
\hline & Minimum corrected weight of impurity in sample $(\mu g)$ : & 31.73 & 5.89 & 37.62 \\
\hline & Maximum corrected weight of impurity in sample $(\mu g)$ : & 32.09 & 6.15 & 38.23 \\
\hline
\end{tabular}

Comments

Checked against official results of analyses for RMAL2014 by FCM on 7/23/2009

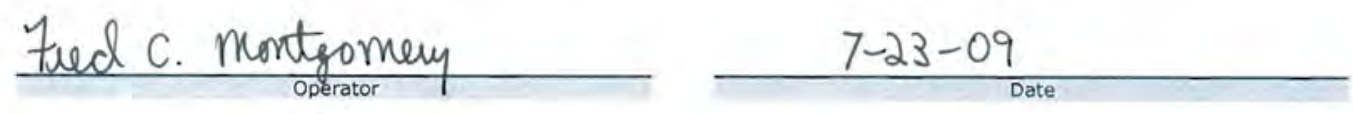


Data Report Form DRF-26A: Measurement of $U$ Contamination or Impurities by Deconsolidation Leach

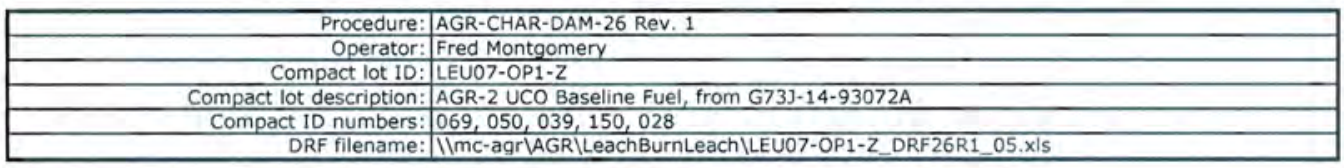

\begin{tabular}{|c|c|c|c|c|}
\hline & $\begin{array}{l}\text { Mean average weight uranium per particle }(g) \text { : } \\
\text { Uncertainty in mean average weight uranium per particle (q): }\end{array}$ & & $\frac{3.93 E-04}{6.56 E-07}$ & \\
\hline & & First Leach & Second Leach & Total \\
\hline & Deconsolidation-leach solution ID: & L09061802 & L09062202 & \\
\hline & Number of compacts: & & & \\
\hline & Total volume of leach solution $(\mathrm{ml})$ : & 141.0 & 130.0 & \\
\hline & Radiochemical laboratory analysis number: & 20140017 & & \\
\hline & Measured uranium concentration $(\mathrm{\mu g} / \mathrm{ml})$ : & $\frac{2014017}{3.44 E-02}$ & $\frac{2014-0.22}{7.78 E-03}$ & \\
\hline & Uncertainty in uranium concentration $(\mathrm{\mu g} / \mathrm{ml})$ : & $3.44 \mathrm{E}-03$ & $7.78 \mathrm{E}-04$ & \\
\hline & Weight uranium leached $(g)$ : & $4.85 \mathrm{E}-06$ & $1.01 \mathrm{E}-06$ & $5.86 E-06$ \\
\hline & Uncertainty in weight uranium leached $(g)$ : & $4.86 \mathrm{E}-07$ & $1.01 \mathrm{E}-07$ & $4.96 \mathrm{E}-07$ \\
\hline & Effective number of exposed kernels: & 0.0 & 0.0 & 0.0 \\
\hline & Uncertainty in effective number of exposed kernels: & 0.0 & 0.0 & 0.0 \\
\hline & Measured concentration of impurity in sample $(\mu \mathrm{g} / \mathrm{ml})$ : & $<4.12 \mathrm{E}-02$ & $<4.12 \mathrm{E}-02$ & $\mathbf{F e}$ \\
\hline & Uncorrected weight of impurity in sample $(\mu \mathrm{g})$ : & $<5.81$ & $<5.36$ & $<11.17$ \\
\hline $\mathrm{Fe}$ & Weight of impurity in blank $(\mu \mathrm{g})$ : & $<7.37$ & $<5.27$ & \\
\hline & Minimum corrected weight of impurity in sample $(\mu \mathrm{g})$ : & 0.00 & 0.00 & 0.00 \\
\hline & Maximum corrected weight of impurity in sample $(\mu g)$ : & 5.81 & 5.36 & 11.17 \\
\hline & Measured concentration of impurity in sample $(\mathrm{\mu g} / \mathrm{ml})$ : & $3.99 \mathrm{E}-03$ & $3.26 \mathrm{E}-03$ & \\
\hline & Uncorrected weight of impurity in sample $(\mu g)$ : & 0.56 & 0.42 & 0.99 \\
\hline $\mathrm{Cr}$ & Weight of impurity in blank $(\mu g)$ : & $<0.36$ & $<0.26$ & \\
\hline & Minimum corrected weight of impurity in sample $(\mu g)$ : & 0.20 & 0.17 & 0.37 \\
\hline & Maximum corrected weight of impurity in sample $(\mu g)$ : & 0.56 & 0.42 & 0.99 \\
\hline & Measured concentration of impurity in sample $(\mathrm{\mu g} / \mathrm{ml})$ : & $<1.91 \mathrm{E}-03$ & $<1,91 \mathrm{E}-03$ & $\mathrm{Mn}$ \\
\hline & Uncorrected weight of impurity in sample $(\mu \mathrm{g})$ : & $<0.27$ & $<0.25$ & $<0.52$ \\
\hline Mn & Weight of impurity in blank $(\mu \mathrm{g})$ : & $<0.34$ & $<0.24$ & \\
\hline & Minimum corrected weight of impurity in sample $(\mu \mathrm{g})$ : & 0.00 & 0.00 & 0.00 \\
\hline & $\begin{array}{l}\text { Maximum corrected weight of impurity in sample }(\mu \mathrm{g}) \text { : } \\
\text { Measured concentration of impurity in sample }(\mu \mathrm{\mu} / \mathrm{ml})\end{array}$ & 0.27 & 0.25 & 0.52 \\
\hline & $\frac{\text { Measured concentration of impurity in sample }(\mu \mathrm{g} / \mathrm{ml}) \text { : }}{\text { Uncorrected weight of impurity in sample }(\mu \mathrm{o}) \text { : }}$ & $<1.62 \mathrm{E}-03$ & $<1.62 \mathrm{E}-03$ & Co \\
\hline Co & $\begin{array}{l}\text { Uncorrected weight of impurity in sample }(\mu g) \text { : } \\
\text { Weight of impurity in blank }(\mu g) \text { : }\end{array}$ & $<0.23$ & $<0.21$ & $<0.44$ \\
\hline & $\begin{array}{l}\text { Weight of impurity in blank }(\mu g) \text { : } \\
\text { Minimum corrected weight of impurity in sample }(\mu g) \text { : }\end{array}$ & $<0.29$ & $<0.21$ & 000 \\
\hline & Maximum corrected weight of impurity in sample $(\mu \mathrm{g})$ : & 0.23 & 0.21 & 0.44 \\
\hline & Measured concentration of impurity in sample $(\mathrm{\mu g} / \mathrm{ml})$ : & $<8.00 \mathrm{E}-03$ & $<8.00 \mathrm{E}-03$ & $\mathrm{Ni}$ \\
\hline & Uncorrected weight of impurity in sample $(\mu g)$ : & $<1.13$ & $<1.04$ & $<2.17$ \\
\hline $\mathbf{N i}$ & Weight of impurity in blank $(\mu \mathrm{g})$ : & $<1.43$ & $<1.02$ & \\
\hline & Minimum corrected weight of impurity in sample $(\mu g)$ : & 0.00 & 0.00 & 0.00 \\
\hline & Maximum corrected weight of impurity in sample $(\mu g)$ : & 1.13 & 1.04 & 2.17 \\
\hline & Measured concentration of impurity in sample $(\mu \mathrm{g} / \mathrm{ml})$ : & $6.65 \mathrm{E}-01$ & $<1.00 \mathrm{E}-01$ & $\mathrm{Ca}$ \\
\hline & Uncorrected weight of impurity in sample $(\mu g)$ : & 93.77 & $<13.00$ & $<106.77$ \\
\hline $\mathrm{Ca}$ & Weight of impurity in blank $(\mu \mathrm{g})$ : & 137.11 & $<12.80$ & \\
\hline & Minimum corrected weight of impurity in sample $(\mu g)$ : & 0.00 & 0.00 & 0.00 \\
\hline & Maximum corrected weight of impurity in sample $(\mu g)$ : & 0.00 & 13.00 & 13.00 \\
\hline & Measured concentration of impurity in sample $(\mathrm{\mu g} / \mathrm{ml})$ : & $3.63 \mathrm{E}-01$ & $8.54 \mathrm{E}-02$ & Al \\
\hline & Uncorrected weight of impurity in sample $(\mu g)$ : & 51.18 & 11.10 & 62.29 \\
\hline Al & Weight of impurity in blank $(\mu \mathrm{g})$ : & $<1.43$ & $<1.02$ & \\
\hline & Minimum corrected weight of impurity in sample $(\mu g)$ : & 49.75 & 10.08 & 59.83 \\
\hline & Maximum corrected weight of impurity in sample $(\mu \mathrm{g})$ : & 51.18 & 11.10 & 62.29 \\
\hline & Measured concentration of impurity in sample $(\mu \mathrm{g} / \mathrm{ml})$ : & $1.40 \mathrm{E}-02$ & $1.67 \mathrm{E}-02$ & $\mathrm{Ti}$ \\
\hline & Uncorrected weight of impurity in sample $(\mu \mathrm{g})$ : & 1.97 & 2.17 & 4.15 \\
\hline $\mathrm{Ti}$ & Weight of impurity in blank $(\mathrm{\mu g})$ : & $<1.43$ & $<1.02$ & \\
\hline & Minimum corrected weight of impurity in sample $(\mu \mathrm{g})$ : & 0.54 & 1.15 & 1,69 \\
\hline & Maximum corrected weight of Impurity in sample $(\mu \mathrm{g})$ : & 1.97 & 2.17 & 4.15 \\
\hline & Measured concentration of impurity in sample $(\mu \mathrm{g} / \mathrm{ml})$ : & $2.02 E-01$ & $6.18 \mathrm{E}-02$ & v \\
\hline & Uncorrected weight of impurity in sample $(\mu \mathrm{g})$ : & 28.48 & 8.03 & 36.52 \\
\hline v & Weight of impurity in blank $(\mu \mathrm{g})$ : & $<0.36$ & $<0.26$ & \\
\hline & Minimum corrected weight of impurity in sample $(\mu g)$ : & 28.12 & 7.78 & 35.90 \\
\hline & Maximum corrected weight of impurity in sample $(\mu \mathrm{g})$ : & 28.48 & 8.03 & 36.52 \\
\hline & Comme & & & \\
\hline check & gainst official results of analyses for RMAL2014 by FCM on 7 & 2009 & & \\
\hline
\end{tabular}
Feed C. Montgonmen
$7-23-09$ 
Data Report Form DRF-26A: Measurement of $U$ Contamination or Impurities by Deconsolidation Leach

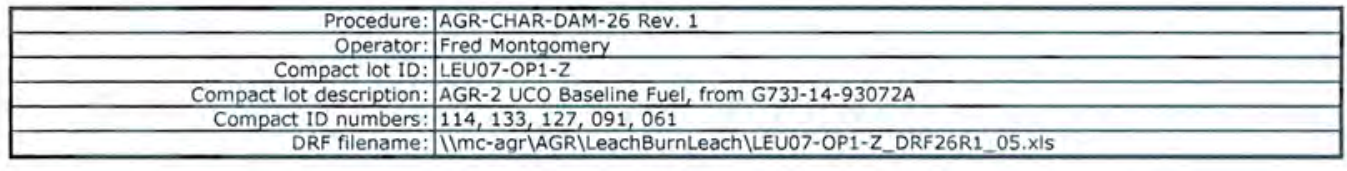

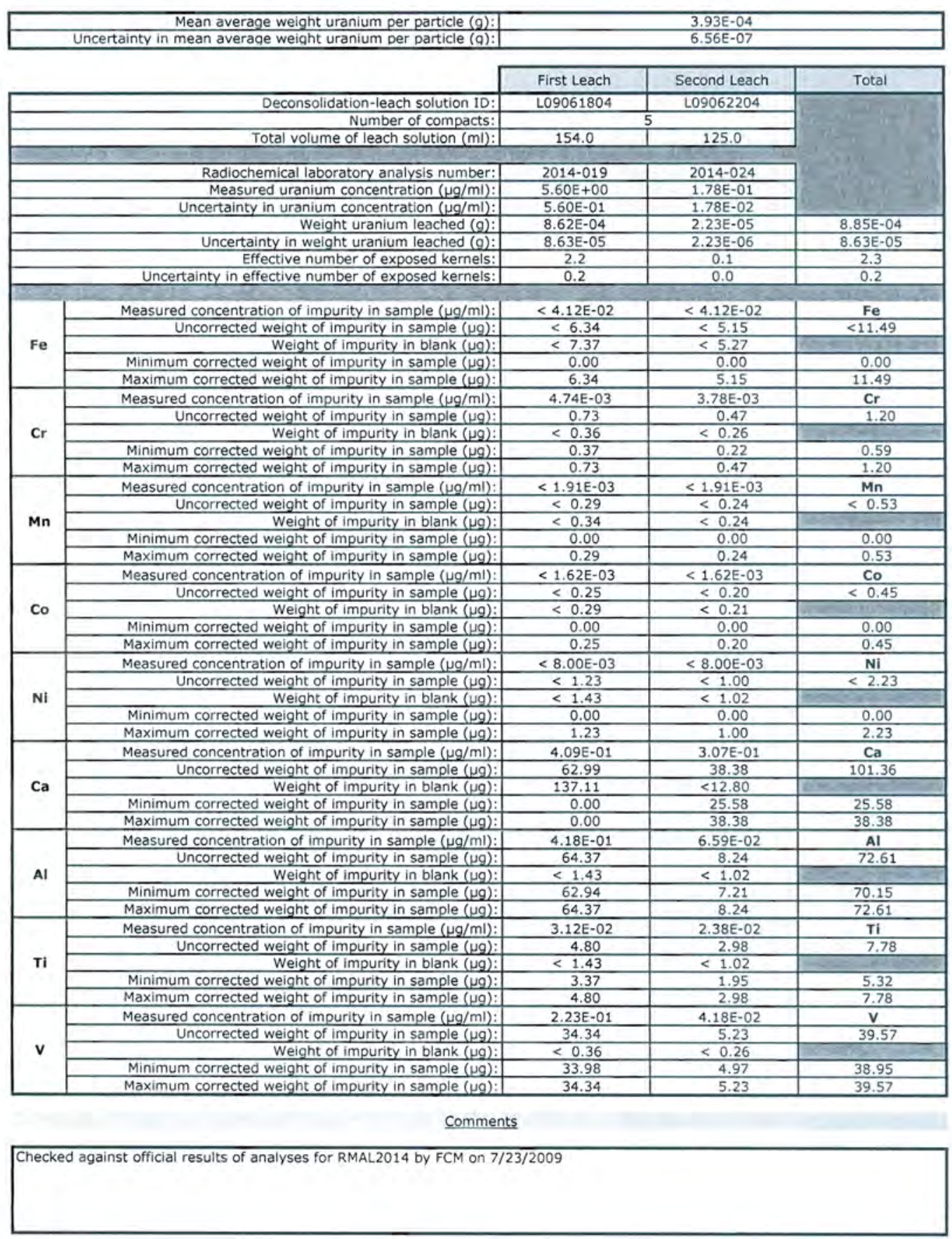

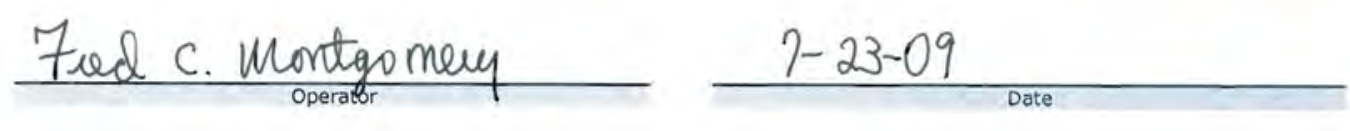


Data Report Form DRF-26A: Measurement of U Contamination or Impurities by Deconsolidation Leach

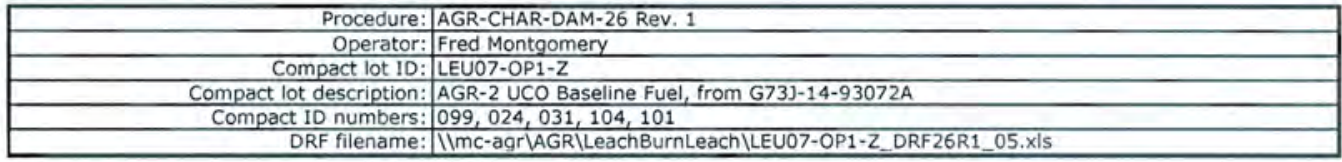

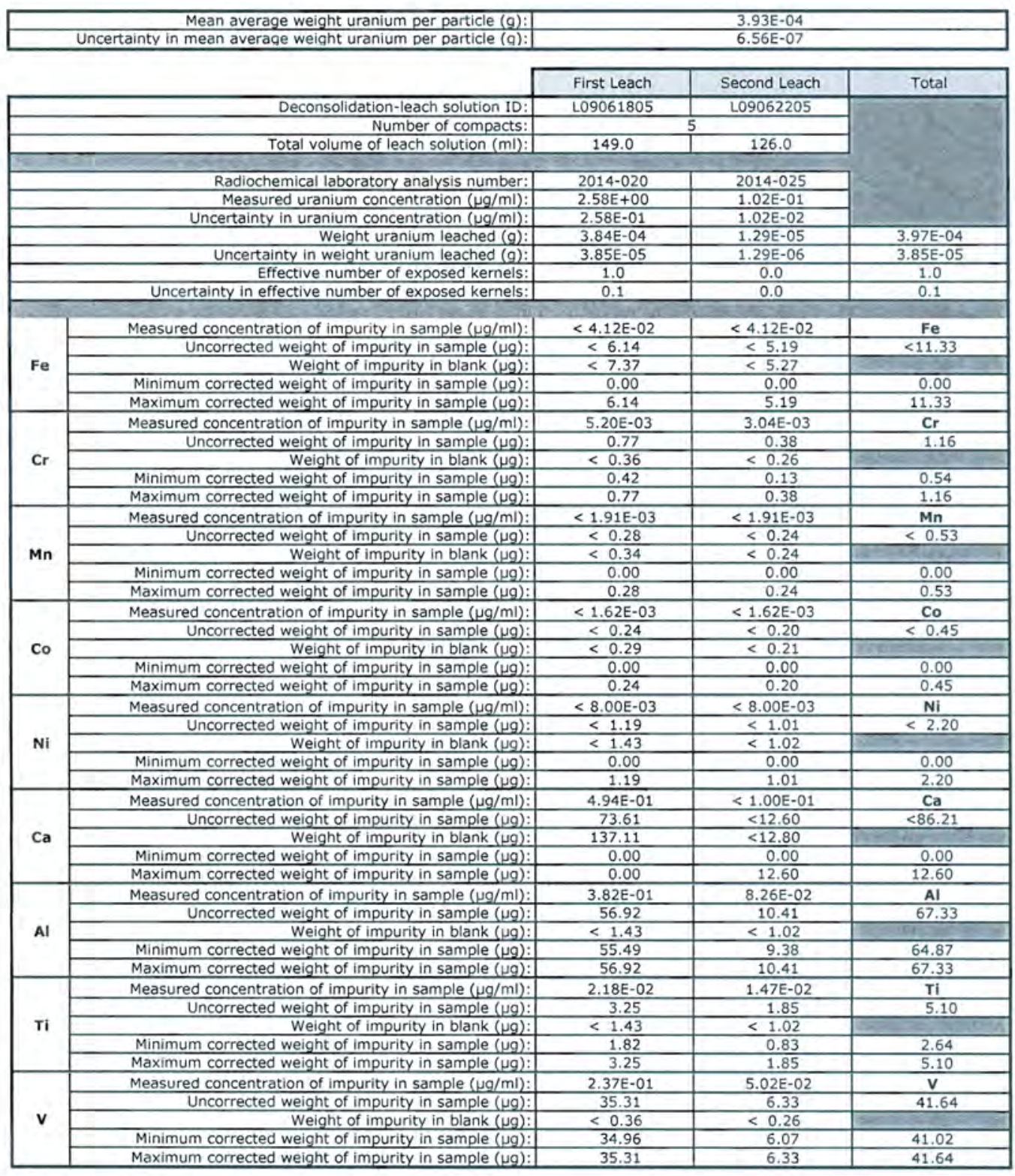

\section{comments}

Checked against official results of analyses for RMAL2014 by FCM on 7/23/2009

\section{Fued c. Mortgomen}

$7-23-09$

Date 
Data Report Form DRF-26A: Measurement of U Contamination or Impurities by Deconsolidation Leach

\begin{tabular}{|c|c|}
\hline Procedure: $A$ & AGR-CHAR-DAM- 26 Rev. 1 \\
\hline Operator: 1 & Fred Montgomery \\
\hline Compact lot ID: 1 & LEU07-OP1-Z \\
\hline Compact lot description: $A$ & AGR-2 UCO Baseline Fuel, from G73J-14-93072A \\
\hline Compact ID numbers: [ & Deconsolidation Leach Blank \\
\hline DRF filename: I & IImc-agr|AGR\LeachBurnLeach|LEU07-OP1-Z_DRF26R1_05.xls \\
\hline
\end{tabular}

\begin{tabular}{|c|c|c|c|c|}
\hline & \multirow[t]{2}{*}{ Uncertainty in mean average weight uranium per particle $(\mathrm{g})$ : } & \multicolumn{3}{|c|}{$\begin{array}{r}3.93 \mathrm{E}-04 \\
6.56 \mathrm{E}-07 \\
\end{array}$} \\
\hline & & First Leach & Second Leach & Total \\
\hline & Deconsolidation-leach solution ID: & L09061803 & L09062203 & \multirow{6}{*}{ 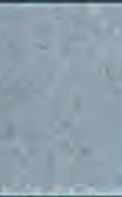 } \\
\hline & Number of compacts: & \multicolumn{2}{|c|}{ None } & \\
\hline & Total volume of leach solution $(\mathrm{ml})$ : & \multirow{2}{*}{179.0} & \multirow{2}{*}{128.0} & \\
\hline \multicolumn{2}{|c|}{ 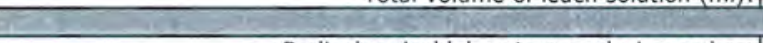 } & & & \\
\hline & $\begin{array}{l}\text { Radiochemical laboratory analysis number: } \\
\text { Measured uranium concentration }(\mu \mathrm{g} / \mathrm{ml}) \text { : }\end{array}$ & $\frac{2014-018}{5.80 \mathrm{E}-04}$ & $\frac{2014-023}{<5.00 E-04}$ & \\
\hline & Uncertainty in uranium concentration $(\mu \mathrm{g} / \mathrm{ml})$ : & $5.80 \mathrm{E}-05$ & & \\
\hline & Weight uranium leached $(\mathrm{g})$ : & $1.04 \mathrm{E}-07$ & $<6.40 \mathrm{E}-08$ & $<1.68 \mathrm{E}-07$ \\
\hline & Uncertainty in weight uranium leached $(g)$ : & $1.04 \mathrm{E}-08$ & & \\
\hline & Effective number of exposed kernels: & 0.0 & 0.0 & 0.0 \\
\hline & Uncertainty in effective number of exposed kernels: & 0.0 & & \\
\hline & & & & \multirow[b]{2}{*}{5} \\
\hline \multirow{2}{*}{$\mathrm{Fe}$} & Measured concentration $(\mu \mathrm{g} / \mathrm{ml})$ : & $<4.12 \mathrm{E}-02$ & $<4.12 \mathrm{E}-02$ & \\
\hline & Total weight of leached impurity $(\mu \mathrm{g})$ : & $<7.37$ & $<5.27$ & $<12.65$ \\
\hline \multirow[b]{2}{*}{$\mathrm{Cr}$} & Measured concentration $(\mu \mathrm{g} / \mathrm{ml})$ : & $<2.00 \mathrm{E}-03$ & $<2.00 \mathrm{E}-03$ & $\mathrm{Cr}$ \\
\hline & Total weight of leached impurity $(\mu \mathrm{g})$ : & $<0.36$ & $<0.26$ & $<0.61$ \\
\hline \multirow{2}{*}{ Mn } & Measured concentration $(\mu \mathrm{g} / \mathrm{ml})$ : & $<1.91 \mathrm{E}-03$ & $<1.91 \mathrm{E}-03$ & \multirow{2}{*}{$\begin{array}{l}\frac{\mathrm{Mn}}{<0.59} \\
\end{array}$} \\
\hline & Total weight of leached impurity $(\mu \mathrm{g})$ : & $<0.34$ & $<0.24$ & \\
\hline \multirow[b]{2}{*}{ Co } & Measured concentration $(\mu \mathrm{g} / \mathrm{ml})$ : & $<1.62 \mathrm{E}-03$ & $<1.62 \mathrm{E}-03$ & \multirow{2}{*}{$\frac{\text { Co }}{<0.50}$} \\
\hline & Total weight of leached impurity $(\mu \mathrm{g})$ : & $<0.29$ & $<0.21$ & \\
\hline \multirow{2}{*}{$\mathrm{Ni}$} & Measured concentration $(\mu \mathrm{g} / \mathrm{ml})$ : & $<8.00 \mathrm{E}-03$ & $<8.00 \mathrm{E}-03$ & \multirow{2}{*}{$\frac{\mathrm{Ni}}{<2,46}$} \\
\hline & Total weight of leached impurity $(\mu \mathrm{g})$ : & $<1.43$ & $<1.02$ & \\
\hline \multirow{2}{*}{$\mathrm{Ca}$} & Measured concentration $(\mu \mathrm{g} / \mathrm{ml})$ : & $7.66 \mathrm{E}-01$ & $<1.00 \mathrm{E}-01$ & \multirow{2}{*}{$\frac{\mathrm{Ca}}{<149.91}$} \\
\hline & Total weight of leached impurity $(\mu \mathrm{g})$ : & 137.11 & $<12.80$ & \\
\hline \multirow{2}{*}{ Al } & Measured concentration $(\mu \mathrm{g} / \mathrm{ml})$ : & $<8.00 \mathrm{E}-03$ & $<8.00 \mathrm{E}-03$ & \multirow{2}{*}{$\begin{array}{c}\mathrm{Al} \\
2.46\end{array}$} \\
\hline & Total weight of leached impurity $(\mu \mathrm{g})$ : & $<1.43$ & $<1.02$ & \\
\hline \multirow{2}{*}{$\mathrm{Ti}$} & Measured concentration $(\mu \mathrm{g} / \mathrm{ml})$ : & $<8.00 \mathrm{E}-03$ & $<8.00 \mathrm{E}-03$ & $\mathrm{Ti}$ \\
\hline & Total weight of leached impurity $(\mu \mathrm{g})$ : & $<1.43$ & $<1.02$ & $<2.46$ \\
\hline \multirow[b]{2}{*}{$\mathbf{v}$} & Measured concentration $(\mu \mathrm{g} / \mathrm{ml})$ : & $<2.00 \mathrm{E}-03$ & $<2.00 \mathrm{E}-03$ & \multirow[b]{2}{*}{$<0.61$} \\
\hline & Total weight of leached impurity $(\mu \mathrm{g})$ : & $<0.36$ & $<0.26$ & \\
\hline
\end{tabular}

Comments

Checked against official results of analyses for RMAL2014 by FCM on 7/23/2009

$\frac{\text { Fued C. Montgomey }}{\text { operator }}$
$1-23-09$ 
Data Report Form DRF-27: Counting of Particles with a Defective OPyC Layer from Deconsolidated Compacts by Visual Inspection

\begin{tabular}{|c|c|}
\hline Procedure: & AGR-CHAR-DAM-27 Rev. 0 \\
\hline Operator: & Fred Montgomery \\
\hline Compact lot ID: & LEU07-OP1-Z \\
\hline Compact lot description: & AGR-2 UCO Baseline Fuel, from G73J-14-93072A \\
\hline Compact ID number: & 079 \\
\hline DRF filename: & 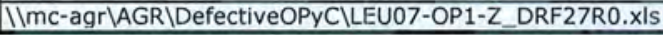 \\
\hline
\end{tabular}

Number of particles with cracked OPyC: 10

Number of particles with partially missing OPyC: 0

Number of particles with completely missing OPyC: 0

Total number of particles with defective OPyC: 0

\section{Comments on unusual visual characteristics of OPyC}

Found 1 particle with a few shallow indents which did not penetrate through the OPyC.

Found 1 particle which has a gouge along the surface of the OPyC.

Fred c. montgonem

$7-2-09$

Operator

Date 
Data Report Form DRF-28: Counting of Particles with Excessive Uranium Dispersion Inside SiC

\begin{tabular}{|c|c|}
\hline Procedure: & AGR-CHAR-DAM-28 Rev. 2 \\
\hline Operator: & John Hunn/Ivan Dunbar/Paul Menchhofer/Jason Ramey \\
\hline Compact lot ID: & LEU07-OP1-Z \\
\hline Compact lot description: & AGR-2 UCO Baseline Fuel, from G73]-14-93072A \\
\hline Compact ID numbers: & 060046122146130141138111064053023076006040034139083087145049 \\
\hline DRF filename: & IImc-agr|AGR\DefectiveIPyCILEU07-OP1-Z_DRF28R2.xIS \\
\hline
\end{tabular}

Number of compacts from which particles were recovered: 20

Weight of sample of particles $(\mathrm{g}): 53.159$

Number of particles in sample: 63538

Mean average weight/particle $(\mathrm{g}): 8.37 \mathrm{E}-04$

Number of particles with excessive U dispersion: 443

\section{Comments}

Severity of uranium dispersion as imaged by $x$-ray radiography varied. Many particles showed severe dispersion in buffer region, with uranium usually piled up at buffer/IPyC interface, but sometimes appearing to extend into IPyC.

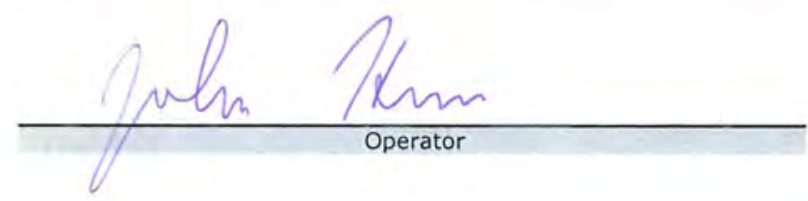

$$
12 \cdot 7-09
$$




\section{For Information Only}

The information in the remainder of this section is from additional characterization that was not required by the fuel product specification.

\section{Anisotropy of pyrocarbon layers after compacting}

To examine the change in pyrocarbon anisotropy during compact fabrication, particles were recovered after deconsolidation of the particles from the compact for defective OPyC analysis. After compacting, the anisotropy of the pyrocarbon layers was observed to increase. This increase occurs during the heat treatment of the compacts at $1800^{\circ} \mathrm{C}$ for 1 hour. The diattenuation of the IPyC increased from $0.0118 \pm 0.0006$ to $0.0150 \pm 0.0006(1.0355 \pm 0.0019$ to $1.0451 \pm 0.0019$ in terms of effective BAFo). The diattenuation of the OPyC increased from $0.0081 \pm 0.0003$ to $0.0129 \pm 0.0007(1.0242 \pm 0.0008$ to $1.0388 \pm 0.0021$ in terms of effective BAFo). The following data report forms contain the data for these measurements. 
Data Report Form DRF-18A: Measurement of Pyrocarbon Anisotropy using the 2-MGEM - IPyC

\begin{tabular}{|c|c|}
\hline Procedure: & AGR-CHAR-DAM-18 Rev. 1 \\
\hline Operator: & G. E. Jellison \\
\hline Mount ID: & M09111301 \\
\hline Sample ID: & LEU07-OP1-Z079 \\
\hline Sample Description: & AGR-2 UCO Baseline Fuel, from G73]-14-93072A, after compacting \\
\hline Folder containing data: & 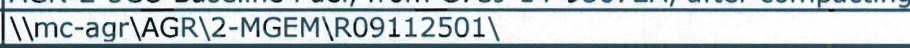 \\
\hline
\end{tabular}

\begin{tabular}{|c|c|c|c|c|c|c|c|}
\hline \multirow{2}{*}{ Particle \# } & \multirow{2}{*}{$\begin{array}{c}\text { Grid } \\
\text { Position }\end{array}$} & \multicolumn{3}{|c|}{ Diattenuation } & \multicolumn{3}{|c|}{ Equivalent $\mathrm{BAFo}=1+3 \mathrm{~N}$} \\
\hline & & Average & St. Dev. & Ave. Error & Average & St. Dev. & Ave. Error \\
\hline 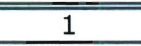 & 4,4 & 0.0154 & 0.0023 & 0.0008 & 1.0462 & 0.0069 & 0.0024 \\
\hline 2 & 4,5 & 0.0147 & 0.0024 & 0.0008 & 1.0441 & 0.0072 & 0.0024 \\
\hline 3 & 4,6 & 0.0146 & 0.0046 & 0.0009 & 1.0438 & 0.0138 & 0.0027 \\
\hline 4 & 5,4 & 0.0154 & 0.0029 & 0.0008 & 1.0462 & 0.0087 & 0.0024 \\
\hline 5 & 5,5 & 0.0147 & 0.0023 & 0.0008 & 1.0441 & 0.0069 & 0.0024 \\
\hline 6 & 5,6 & 0.0160 & 0.0029 & 0.0008 & 1.0480 & 0.0087 & 0.0024 \\
\hline 7 & 6,4 & 0.0150 & 0.0028 & 0.0009 & 1.0450 & 0.0084 & 0.0027 \\
\hline 8 & 6,5 & 0.0160 & 0.0026 & 0.0008 & 1.0480 & 0.0078 & 0.0024 \\
\hline 9 & 6,6 & 0.0143 & 0.0023 & 0.0009 & 1.0429 & 0.0069 & 0.0027 \\
\hline 10 & 5,7 & 0.0142 & 0.0021 & 0.0009 & 1.0426 & 0.0063 & 0.0027 \\
\hline \multicolumn{2}{|c|}{ Average } & 0.0150 & 0.0027 & 0.0008 & 1.0451 & 0.0082 & 0.0025 \\
\hline
\end{tabular}

Mean of average BAFo per particle: 1.0451

Standard deviation of average BAFo per particle: 0.0019

\section{Comments}

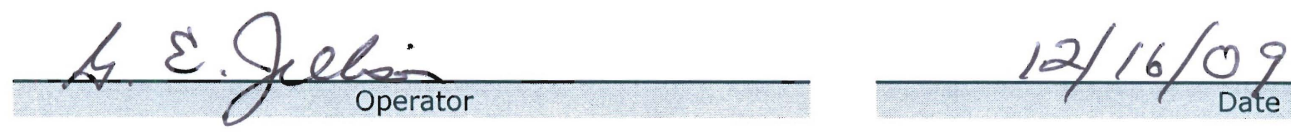


Data Report Form DRF-18B: Measurement of Pyrocarbon Anisotropy using the 2-MGEM - OPyC

\begin{tabular}{|r|l|}
\hline Procedure: & AGR-CHAR-DAM-18 Rev. 1 \\
\hline Operator: & G. E. Jellison \\
\hline Mount ID: & M09111301 \\
\hline Sample ID: & LEU07-OP1-Z079 \\
\hline Sample Description: & AGR-2 UCO Baseline Fuel, from G73J-14-93072A, after compacting \\
\hline Folder containing data: & $\backslash \backslash \mathrm{MC}$-agr\AGR\2-MGEM\R09112501\} $\\
{\hline}$
\end{tabular}

\begin{tabular}{|c|c|c|c|c|c|c|c|}
\hline \multirow{2}{*}{ Particle \# } & \multirow{2}{*}{$\begin{array}{c}\text { Grid } \\
\text { Position }\end{array}$} & \multicolumn{3}{|c|}{ Diattenuation } & \multicolumn{3}{|c|}{ Equivalent $\mathrm{BAFo}=1+3 \mathrm{~N}$} \\
\hline & & Average & St. Dev. & Ave. Error & Average & St. Dev. & Ave. Error \\
\hline 1 & 4,4 & 0.0137 & 0.0027 & 0.0010 & 1.0411 & 0.0081 & 0.0030 \\
\hline 2 & 4,5 & 0.0132 & 0.0028 & 0.0010 & 1.0396 & 0.0084 & 0.0030 \\
\hline 3 & 4,6 & 0.0133 & 0.0029 & 0.0012 & 1.0399 & 0.0087 & 0.0036 \\
\hline 4 & 5,4 & 0.0135 & 0.0032 & 0.0011 & 1.0405 & 0.0096 & 0.0033 \\
\hline 5 & 5,5 & 0.0113 & 0.0025 & 0.0010 & 1.0339 & 0.0075 & 0.0030 \\
\hline 6 & 5,6 & 0.0131 & 0.0026 & 0.0011 & 1.0393 & 0.0078 & 0.0033 \\
\hline 7 & 6,4 & 0.0126 & 0.0028 & 0.0011 & 1.0378 & 0.0084 & 0.0033 \\
\hline 8 & 6,5 & 0.0134 & 0.0027 & 0.0010 & 1.0402 & 0.0081 & 0.0030 \\
\hline 9 & 6,6 & 0.0124 & 0.0026 & 0.0010 & 1.0372 & 0.0078 & 0.0030 \\
\hline 10 & 5,7 & 0.0127 & 0.0025 & 0.0011 & 1.0381 & 0.0075 & 0.0033 \\
\hline \multicolumn{2}{|c|}{ Average } & 0.0129 & 0.0027 & 0.0011 & 1.0388 & 0.0082 & 0.0032 \\
\hline
\end{tabular}

Mean of average BAFo per particle: 1.0388

Standard deviation of average BAFo per particle: 0.0021

\section{Comments}
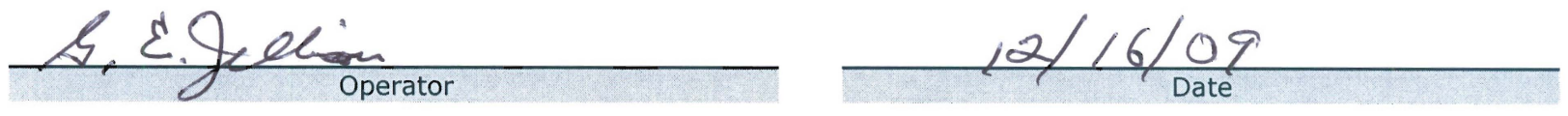
Data Report Form DRF-18A: Measurement of Pyrocarbon Anisotropy using the 2-MGEM - IPyC

\begin{tabular}{|c|c|}
\hline Procedure: & AGR-CHAR-DAM-18 Rev. 1 \\
\hline Operator: & G. E. Jellison \\
\hline Mount ID: & M09111301 \\
\hline Sample ID: & LEU07-OP1-Z079 \\
\hline Sample Description: & AGR-2 UCO Baseline Fuel, from G73J-14-93072A, after compacting \\
\hline Folder containing data: & 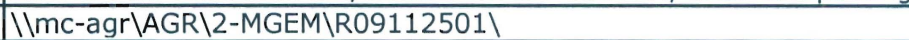 \\
\hline
\end{tabular}

\begin{tabular}{|c|c|c|c|c|c|c|c|}
\hline \multirow{2}{*}{ Particle \# } & \multirow{2}{*}{$\begin{array}{c}\text { Grid } \\
\text { Position }\end{array}$} & \multicolumn{3}{|c|}{ Diattenuation } & \multicolumn{3}{|c|}{ True BAFo $=(1+N) /(1-N)$} \\
\hline & & Average & St. Dev. & Ave. Error & Average & St. Dev. & Ave. Error \\
\hline 1 & 4,4 & 0.0154 & 0.0023 & 0.0008 & 1.0313 & 0.0047 & 0.0017 \\
\hline 2 & 4,5 & 0.0147 & 0.0024 & 0.0008 & 1.0298 & 0.0049 & 0.0016 \\
\hline 3 & 4,6 & 0.0146 & 0.0046 & 0.0009 & 1.0296 & 0.0095 & 0.0019 \\
\hline 4 & 5,4 & 0.0154 & 0.0029 & 0.0008 & 1.0313 & 0.0060 & 0.0017 \\
\hline 5 & 5,5 & 0.0147 & 0.0023 & 0.0008 & 1.0298 & 0.0047 & 0.0016 \\
\hline 6 & 5,6 & 0.0160 & 0.0029 & 0.0008 & 1.0325 & 0.0060 & 0.0017 \\
\hline 7 & 6,4 & 0.0150 & 0.0028 & 0.0009 & 1.0305 & 0.0058 & 0.0019 \\
\hline 8 & 6,5 & 0.0160 & 0.0026 & 0.0008 & 1.0325 & 0.0054 & 0.0017 \\
\hline 9 & 6,6 & 0.0143 & 0.0023 & 0.0009 & 1.0290 & 0.0047 & 0.0019 \\
\hline 10 & 5,7 & 0.0142 & 0.0021 & 0.0009 & 1.0288 & 0.0043 & 0.0019 \\
\hline \multicolumn{2}{|c|}{ Average } & 0.0150 & 0.0027 & 0.0008 & 1.0305 & 0.0056 & 0.0017 \\
\hline
\end{tabular}

Mean of average BAFo per particle: 1.0305

Standard deviation of average BAFo per particle: 0.0013

\section{Comments}
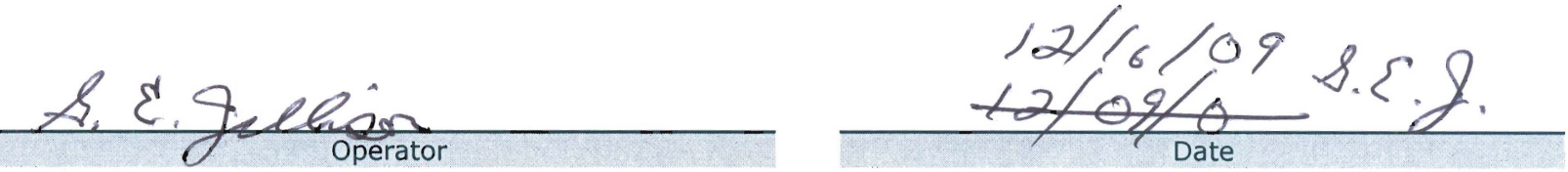
Data Report Form DRF-18B: Measurement of Pyrocarbon Anisotropy using the 2-MGEM - OPyC

\begin{tabular}{|r|l|}
\hline Procedure: & AGR-CHAR-DAM-18 Rev. 1 \\
\hline Operator: & G. E. Jellison \\
\hline Mount ID: & M09111301 \\
\hline Sample ID: & LEU07-OP1-Z079 \\
\hline Sample Description: & AGR-2 UCO Baseline Fuel, from G73J-14-93072A, after compacting \\
\hline Folder containing data: & $\backslash \backslash \mathrm{MC}$-agr\AGR $\backslash 2-M G E M \backslash R 09112501 \backslash$ \\
\hline
\end{tabular}

\begin{tabular}{|c|c|c|c|c|c|c|c|}
\hline \multirow{2}{*}{ Particle \# } & \multirow{2}{*}{$\begin{array}{c}\text { Grid } \\
\text { Position }\end{array}$} & \multicolumn{3}{|c|}{ Diattenuation } & \multicolumn{3}{c|}{ True BAFo $=(1+\mathrm{N}) /(1-\mathrm{N})$} \\
\cline { 3 - 8 } & Average & St. Dev. & Ave. Error & Average & St. Dev. & Ave. Error \\
\hline \hline 1 & 4,4 & 0.0137 & 0.0027 & 0.0010 & 1.0278 & 0.0056 & 0.0021 \\
\hline 2 & 4,5 & 0.0132 & 0.0028 & 0.0010 & 1.0268 & 0.0058 & 0.0021 \\
\hline 3 & 4,6 & 0.0133 & 0.0029 & 0.0012 & 1.0270 & 0.0060 & 0.0025 \\
\hline 4 & 5,4 & 0.0135 & 0.0032 & 0.0011 & 1.0274 & 0.0066 & 0.0023 \\
\hline 5 & 5,5 & 0.0113 & 0.0025 & 0.0010 & 1.0229 & 0.0051 & 0.0020 \\
\hline 6 & 5,6 & 0.0131 & 0.0026 & 0.0011 & 1.0265 & 0.0053 & 0.0023 \\
\hline 7 & 6,4 & 0.0126 & 0.0028 & 0.0011 & 1.0255 & 0.0057 & 0.0023 \\
\hline 8 & 6,5 & 0.0134 & 0.0027 & 0.0010 & 1.0272 & 0.0055 & 0.0021 \\
\hline 9 & 6,6 & 0.0124 & 0.0026 & 0.0010 & 1.0251 & 0.0053 & 0.0021 \\
\hline 10 & 5,7 & 0.0127 & 0.0025 & 0.0011 & 1.0257 & 0.0051 & 0.0023 \\
\hline \multicolumn{2}{|r|}{ Average } & 0.0129 & 0.0027 & 0.0011 & 1.0262 & 0.0056 & 0.0022 \\
\hline
\end{tabular}

Mean of average BAFo per particle: 1.0262

Standard deviation of average BAFo per particle: 0.0014

\section{Comments}

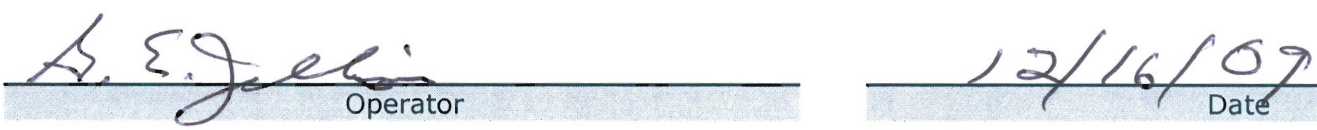




\section{Appendix A: Certificate of Conformance}

This section contains the Certificate of Conformance for the LEU07-OP1-Z compact lot, This is a record of the review by Quality Assurance personnel that specified requirements have been met or that nonconformances to those requirements have been documented. Appendix B contains copies of the applicable Nonconformance Reports. 
Oak Ridge National Laboratory

Advanced Gas Reactor Fuel Development and Qualification Program

CERTIFICATE OF CONFORMANCE

1. ITEM IDENTIFICATION: AGR Fuel Compacts

2. PART LOT AND LOT NUMBER: AGR-2 UCO Baseline, LEU07-OP1-Z

3. PRODUCT DEFINITION: INL Document \#SPC-923, Revision 3 entitled AGR-2 Fuel Specification

4. LIST OF APPROVED DEVIATIONS: Not applicable

\begin{tabular}{|c|c|c|c|c|c|c|c|c|c|}
\hline $\begin{array}{l}\text { *Part } \\
\text { Type }\end{array}$ & $\begin{array}{c}\text { Unique Part } \\
\text { I.D. No. }\end{array}$ & QTY & INIT. & Date & $\begin{array}{l}\text { *Part } \\
\text { Type }\end{array}$ & $\begin{array}{l}\text { Unique Part } \\
\text { I.D. No. }\end{array}$ & QTY & INIT. & Date \\
\hline $\mathrm{FC}$ & 003 & 1 & Df: & $10 / 2310$ & FC & 081 & 1 & 301 & $22 / 231$ \\
\hline $\mathrm{FC}$ & 004 & 1 & & & $\mathrm{FC}$ & 082 & 1 & & \\
\hline $\mathrm{FC}$ & 007 & 1 & & & $\mathrm{FC}$ & 084 & 1 & 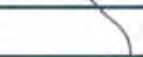 & \\
\hline $\mathrm{FC}$ & 008 & 1 & & & FC & 089 & 1 & & \\
\hline $\mathrm{FC}$ & 012 & 1 & & & $\mathrm{FC}$ & 093 & 1 & & \\
\hline FC & 014 & 1 & & & $\mathrm{FC}$ & 094 & 1 & & \\
\hline $\mathrm{FC}$ & 015 & 1 & & & $\mathrm{FC}$ & 098 & 1 & & \\
\hline FC & 016 & 1 & & & $\mathrm{FC}$ & 102 & 1 & & ) \\
\hline $\mathrm{FC}$ & 019 & 1 & & & $\mathrm{FC}$ & 105 & 1 & & \\
\hline FC & 020 & 1 & & & $\mathrm{FC}$ & 106 & 1 & & \\
\hline FC & 021 & 1 & & & FC & 109 & 1 & & \\
\hline $\mathrm{FC}$ & 036 & 1 & & & $\mathrm{FC}$ & 113 & 1 & & \\
\hline $\mathrm{FC}$ & 041 & 1 & & & $\mathrm{FC}$ & 116 & 1 & & \\
\hline $\mathrm{FC}$ & 043 & 1 & & & $\mathrm{FC}$ & 117 & 1 & & \\
\hline FC & 054 & 1 & & & FC & 118 & 1 & & \\
\hline $\mathrm{FC}$ & 063 & 1 & & & $\mathrm{FC}$ & 121 & 1 & & \\
\hline FC & 065 & 1 & & & $\mathrm{FC}$ & 126 & 1 & & \\
\hline $\mathrm{FC}$ & 067 & 1 & & & $\mathrm{FC}$ & 128 & 1 & & \\
\hline $\mathrm{FC}$ & 068 & 1 & & & FC & 131 & 1 & & \\
\hline FC & 071 & 1 & 7 & & $\mathrm{FC}$ & 132 & 1 & & \\
\hline FC & 072 & 1 & & 7 & FC & 137 & 1 & & \\
\hline $\mathrm{FC}$ & 073 & 1 & & & $\mathrm{FC}$ & 143 & 1 & & \\
\hline $\mathrm{FC}$ & 074 & 1 & & & $\mathrm{FC}$ & 148 & 1 & & \\
\hline $\mathrm{FC}$ & 080 & 1 & 1 & $\zeta$ & $\mathrm{FC}$ & 151 & 1 & & 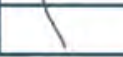 \\
\hline
\end{tabular}

5. LIST OF APPLICABLE NONCONFORMANCE REPORT NUMBERS (NCRs attached in Appendix B of data package): NCR-X-MSTD-AGR-10-01, INL NCR 44791, INL NCR 44792

With the exception of the Deviations documented on the forms referenced in Item 4 and the nonconforming conditions documented on Nonconformance Reports referenced in Item 5, the listed parts have been produced and tested in compliance to the requirements of the QAP for the AGR Program at ORNL

(Document \# QAP-ORNL-AGR-01), its subordinate implementing procedures, and to the specified product definition prescribed in the document(s) referenced in Item 3.

M. C. Vance, AGR Quality Representative,

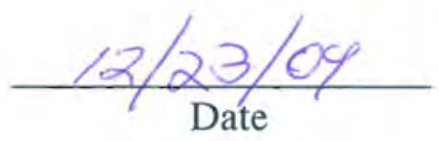

Materials Science and Technology Division, ORNL

* FC indicates fuel compact 


\section{Appendix B: Nonconformance Reports}

This section contains the applicable Nonconformance Reports for the LEU07-OP1-Z compact lot. A nonconformance related to compact length was determined to be acceptable. However, a higher than allowed fraction of exposed uranium was determined to not be acceptable for the AGR-2 irradiation test. The exposed uranium was due to cracked TRISO layers in the coated particle composite. These cracks are thought to have occurred at $\mathrm{B} \& \mathrm{~W}$ when particles were removed from the coating furnace using a suction device. In addition, a higher than allowed IPyC defect fraction was determined to not be acceptable for the AGR-2 irradiation test. Excessive permeability of chlorine through the IPyC during $\mathrm{SiC}$ deposition was evident from $\mathrm{x}-$ ray observation of uranium dispersion out of the kernel and into the buffer after particles experienced $1800^{\circ} \mathrm{C}$ heat treatment in the final stage of compacting. The final disposition of this compact lot was to not use the compacts for the AGR-2 irradiation test, but to retain the compacts in storage at ORNL and INL for possible future analysis or methods development. This disposition was documented on INL NCR-44791 and INL NCR-44792. 


\section{ORNL NONCONFORMANCE REPORT (NCR)}

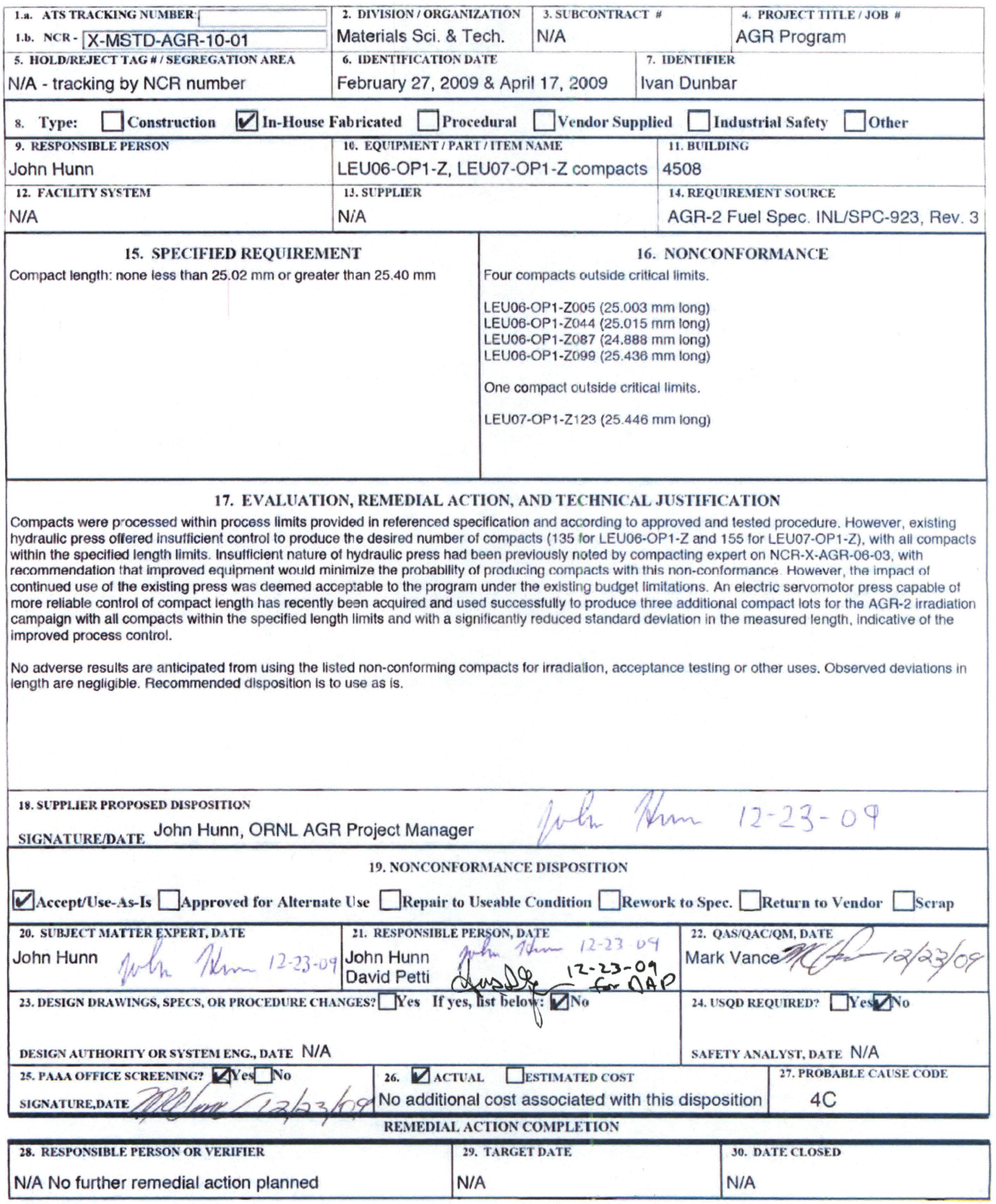




\section{ORNL NONCONFORMANCE REPORT INSTRUCTIONS}

The NCR Form shall be completed by the Responsible Person or designee.

Enter "N/A" in fields not used. Required fields are identified by *.

1.a. *Assessment Tracking System - ATS - Automatic number supplied by the ATS

1.b. *Nonconformance Report - NCR - Number assigned by the appropriate Division/Organization (eg., NCR02-DIV-XXX).

2. Division/Organization - Name or division/organization/program responsible for the NCR.

3. Subcontract \# - For procured items, enter the subcontract or purchase order number

4. Project Title/Job Number - Project title or job number as reflected on drawings, specifications, or other documentation associated with the nonconformance.

5. Hold/Reject Tag \#/Segregation Area - Enter the hold or reject tag number and/or identify the segregation area, as applicable.

6. Identification Date - Date the nonconformance was identified.

7. *Identifier - Name of person initially identifying nonconformance.

8. *Type-Check the type of nonconformance.

9. *Responsible Person - RP - The ORNL line or project personnel responsible for control or use of the item, material or Service in question. The RP is responsible for ensuring analysis and disposition is completed.

10. Equipment/Part/Item Name - Equipment, part or item name as reflected on drawings, specifications, or other documentation.

11. Building - Building number.

12. Facility System - Provide name of facility system.

13. Supplier - Name of vendor, shop, subcontractor, customer, fabricator, or other source supplying nonconforming item, material or service deliverable.

14. *Requirement Source - Indicate the drawings, specifications, procedures, statement of work or subcontract associated with the nonconforming equipment, part, item or service deliverable.

15. *Specified Requirements - Identify the requirements associated with the nonconforming condition.

16. *Nonconformance - Identify the nonconforming condition in detail, with equipment numbers and/or other unique identifiers to clearly identify the equipment, part, item, or service, as applicable.

17. *Evaluation, Remedial Action, and Technical Justification - State the justification for the disposition selected and the remedial action(s) to be taken to make the equipment, part, or item usable. If "use-as-is" or "repair" is determined, then include a technical justification. Identify individual(s) responsible for any remedial actions

18. Supplier Proposed Disposition Signature - Signature of the supplier representative proposing a disposition for ORNL's approval, if applicable.

19. *Nonconformance Disposition - Check the nonconformance disposition.

20. Subject Matter Expert - SME - Signature if SME was used in determining disposition

21. *Responsible Person - RP - Signature of the ORNL line or project person responsible for control or use of the of item, material or service subcontract. Signature indicates "approval" of the proposed disposition

22. *QAS/QAC/QM - Signature of quality representative indicating concurrence with the disposition and that the NCR process was followed.

23. Design drawings, specifications, or procedure changes - List any documents requiring revision. For nuclear facilities only, check and obtain dated signature of design engineer or system engineer.

24. Unresolved Safety Question Determination - USQD - Check and obtain dated signature of safety analyst, if required. (Nuclear Facilities Only)

25. *Price Anderson Amendments Act - P-AAA Screening - Check yes or no based on screening conducted in Step 5 of procedure. Include signature of the person performing the evaluation.

26. Estimated Cost - Estimated or actual cost impact of nonconformance. Identify if the entry is "actual" or "estimated". 
27. Probable Cause Code - Cause Code Selected from ATS:

1 - Equipment/Material Problem. An event or condition resulting from the failure, malfunction, or deterioration of equipment or parts, including instruments or material.

1A - Defective or Failed Part. A part/instrument that lacks something essential to perform its intended function.

1B - Defective or Failed Material. A material defect or failure.

1C - Defective Weld, Braze, or Soldered Joint. A specific weld/joint defect or failure.

1D - Error by Manufacturer in Shipping or Marking. An error by the manufacturer or supplier in the shipping or marking of equipment.

$1 E$ - Electrical or Instrument Noise. An unwanted signal or disturbance that interferes with the operation of equipment.

1F - Contaminant. Failure or degradation due to radiation damage or foreign material such as dirt, crud, or impurities.

$1 G$ - End of Life Failure. A failure where the equipment or material is run to failure and has reached its end of design life.

2 - Procedure Problem. An event or condition that can be traced to the lack of a procedure, an error in a procedure, or a procedural deficiency or inadequacy.

2A - Defective or Inadequate Procedure. A procedure that either contains an error or lacks something essential to the successful performance of the activity.

2B - Lack of Procedure. No written procedure was in place to perform the activity.

3 - Personnel Error. An event or condition due to an error, mistake, or oversight.

$3 \mathrm{~A}$ - Inattention to Detail. Inadequate attention to the specific details of the task.

3B - Procedure Not Used or Used Incorrectly. The failure to use or the inappropriate use of written instructions, procedures, or other documentation.

3C - Communication Problem. Inadequate presentation or exchange of information.

3D - Other Human Error. Human error other than those described above.

4 - Design Problem. An event or condition that can be traced to a defect in design or other factors related to configuration, engineering, layout, tolerances, calculations, etc.

4A - Inadequate Work Environment. Inadequate design of equipment used to communicate information from the facility to a person (e.g., displays, labels, etc.) as well as inadequate work environment, such as inadequate lighting, working space, or other human factor considerations.

4B - Inadequate or Defective Design. A design in which something essential was lacking (defective) or when a detail was included but was not adequate for the requirement (inadequate).

4C - Error in Equipment or Material Selection. A mistake in the equipment or material selection only, not to include a procurement error (see Personnel Error - (e) Other Human Error) or a specification error (see Design Problem - (d) Drawing, Specification, or Data Errors).

4D - Drawing, Specification, or Data Errors. An error in the calculation, information, or specification of a design.

5 - Training Deficiency. An event or condition that can be traced to a lack of training or insufficient training to enable a person to perform a desired task adequately.

5A - No Training Provided. A lack of appropriate training.

5B - Insufficient Practice or Hands-On Experience. An inadequate amount of preparation before performing the activity.

5C - Inadequate Content. The knowledge and skills required to perform the task or job were not identified.

5D - Insufficient Refresher Training. The frequency of refresher training was not sufficient to maintain the required knowledge and skills.

5E - Inadequate Presentation or Materials. The training presentation or materials were insufficient to provide adequate instruction. 
6 - Management Problem. An event or condition that can be directly traced to managerial actions or methods.

6A - Inadequate Administrative Control. A deficiency in the controls in place to administer and direct activities.

6B - Work Organization/Planning Deficiency. A deficiency in the planning, scoping, assignment, or scheduling of work.

6C - Inadequate Supervision. Inadequate techniques used to direct workers in the accomplishment of tasks.

6D - Improper Resource Allocation. Improper personnel or material allocation resulting in the inability to successfully perform assigned tasks.

6E - Policy Not Adequately Defined, Disseminated, or Enforced. Inadequate description, distribution, or enforcement of policies and expectations.

6F - Other Management Problem. A management problem other than those defined above.

7 - External Phenomena. An event or condition caused by factors that are not under the control of the reporting organization or the suppliers of the failed equipment or service.

7 A - Weather or Ambient Condition. Unusual weather or ambient conditions, including hurricanes, tornadoes, flooding, earthquake, and lightning.

7B - Power Failure or Transient. Special cases of power loss that are attributable to outside supplied power.

7C - External Fire or Explosion. An external fire, explosion, or implosion.

7D - Theft, Tampering, Sabotage, or Vandalism. Theft, tampering, sabotage, or vandalism that could not have been prevented by the reporting organization.

8 - Radiological/Hazardous Material Problem. An event related to radiological or hazardous material contamination that cannot be attributed to any of the other causes.

8A - Legacy Contamination. Radiological or hazardous material contamination attributed to past practices. 8B - Source Unknown. Radiological or hazardous material contamination where the source cannot be reasonably determined.

28. Responsible Person or Verifier - Signature closes the NCR and releases the nonconforming item/material/service for further processing.

29. Target Date - Scheduled date for closure of NCR remedial actions..

30. Date closed - Actual date closed. 
230.01

Revision date

09/30/2003

\section{Control of Nonconforming Items Nonconformance Documentation}
Initlator:
Barnes, Charles M
Work Org.:
C700
Work Phone:
059914
6-0864

Documentation

\begin{tabular}{|c|c|}
\hline $\begin{array}{l}\text { NCR Number: } \\
\mathbf{4 4 7 9 1}\end{array}$ & $\begin{array}{l}\text { Date Identified; } \\
\text { 08/04/2009 }\end{array}$ \\
\hline
\end{tabular}

*Item Name:

LEUO6 compacts containing B\&W G73J-14-93074A particles

and other AGR-2 compact lots (LEU07 and possibly LEUO9

and/or LEU11) containing B\&W AGR-2 particles * SSC:
AGR-2 compacts and coated
particles

\section{${ }^{\star}$ Facility:OFF-S}

*Location:ORNL Description:Bldg 4508 and possibly other ORNL buildings

Req. No/P.O. No./SC and/or Project No.:

Project \#23841; Contract \#27240 with B\&W for Industrial fuel fabrication and development; Contract 59613 with ORNL whlch includes AGR compact fabrication and characterization

Supplier Name/Address:

Supplier of AGR-2 particles is Babcock \& Wilcox Co., $1570 \mathrm{Mt}$

Athos Road, Lynchburg, VA 24504

*This NCR is for:

ICP $\triangle I N L \square$ Other

* Is the non-conformance under the requirement of SNF or NRC-licensed activitles (DOE/RW-0333P)? $O$ Yes $O$ No

* Specification to which Item does not perform:

SPC-923, Rev. 2 AGR-2 Fuel Specification (in effect when AGR-2 UCO particle data package was submilted by B\&W) and SPC-923, Rev 3 (In effect when LEU06 and other AGR-2 compacts were characterized)

Associated Documents:

Data Packages for LEU06, LEU07, LEU09 and LEU11 compacts (not issued at this time); B\&W Data Packages for AGR-2 UCO particles, lots G73J-14-93071A, G73J-14-93072A, G73J-14-93073A, G73J-14-93074A \& G73H-10-93085B; TCT meeting notes of March $2 \& 5$ teleconferences, March 16 \& 18 teleconferences, and April 2 teleconference

*Non-Conformance Description:

LEUO6 compacts were found to contain uranium contamination at approximately $10 \mathrm{-4} \mathrm{g}$ exposed $\mathrm{U}$ per gram total $\mathrm{U}$ in compacts, compared to the specification of $\leq 2 \times 10-5 \mathrm{~g}$ exposed $\mathrm{U}$ per $\mathrm{g} U$ in compacts. Exposed uranium lri compacts was determined to be caused by cracks through all layers of the coatings of a fraction of particles contalned in these compacts. Based on several teleconferences of the VHTR TDO Fuels Technical Coordination Team (held on March 2, 5, 16, 18 and Aprll

2), it was recommended that LEU06 compacts not be used in the AGR-2 experiment because of the high uranlum

contamination but replaced by a new set of compacts containing G73J-14-93073A particles. This replacement batch of compacts is expected to have a lower fraction of uranium contamination ( $44 \%$ of the LEU06 fractlon based on all leach and bum leach results and $95 \%$ confidence values or $33 \%$ based on all leach and burn leach results and $50 \%$ confidence values). LEU07 compacts were also found to have uranium contamination above the specification limit, although for a separate reason (urnajum dispersion), LEU07 compacts have been rejected for use in the AGR-2 experiment. The actual uranium contamination values for LEU06 compacts are $\leq 1.4 \mathrm{E}-4$ (95\% confidence based on analysis of 40 compacts only) for LEU06 and $\leqslant 6.9 \times 10-5$ (95\% confidence based on analysis of 100 compacts) for LEU07 compacts. The expecled value for LEU09 compacts is $5 \times 10-5$ (95\% confidence), based on measurements of defect fractions of 217,000 particles from batch $93073 \mathrm{~A}$.

\begin{tabular}{ll|l}
\hline Responsible Manager (RM): & Responsible Quality Engineer (QE): \\
Cox, John R & Roberts, Gary D
\end{tabular}

Allemate RM for processing NCR:

Croson, Dlane $\mathrm{V}$

Next Activity: Implementation Completion - RM

Actlonee: Croson, Diane V

Date Due:

Screening - Responsible Manager

\begin{tabular}{l|l|l|l}
\hline $\begin{array}{l}\text { Responsible Manager (RM): } \\
\text { Croson, Diane V }\end{array}$ & $\begin{array}{l}\text { Organlzation } \\
\text { C700 }\end{array}$ & $\begin{array}{l}\text { Phone: } \\
6-3402\end{array}$ & $\begin{array}{l}\text { Date Screened: } \\
12 / 08 / 2009\end{array}$ \\
\hline
\end{tabular}

\footnotetext{
*Initlator has selected "No" to the non-conformance under the requirement of SNF or NRC-licensed activities
} 
(DOE/RW-0333P). Is this Correct?

Yes $O$ No

* Does the NCR require Stop Work?

Yes No

*Does this NCR support Environmental Requirements? $\bigcirc$ Yes No

RM Comments:

None

RM Change History:

12/08/2009 07:26 AM : Angela J Smith changed the RM from Cox, John R to Croson, Diane V

Screening - Quality Engineer

\begin{tabular}{l|l|l|l}
\hline Quality Engineer (QE): & Organization & $\begin{array}{l}\text { Phone: } \\
6-8961\end{array}$ & $\begin{array}{l}\text { Date Screened: } \\
\text { Roberts, Gary D }\end{array}$ \\
W560 & $6 / 2009$ \\
\hline
\end{tabular}

* is the NCR valid? $O$ Yes $O$ No

Quality Comments:

None

Notification - RM

Responsible Manager (RM):

Croson, Diane $V$

Organization C700

6-8961

$12 / 08 / 2009$

ORPS Report Number:

N/A

*Area of Responsibility:

INL NGNP

Cognizant Director:

Soto, Rafael

Cognizant Director's Alternate(s):

Smith, Angela J; Armour, Kimberly Jo

Compllance Coordinator(s) to determine Price Anderson

(PAAA) noncompliance:

Smith, Angela J

\begin{tabular}{|c|c|}
\hline $\begin{array}{l}\text { Phone: } \\
\text { 6-3402 }\end{array}$ & $\begin{array}{l}\text { Date Notified: } \\
\text { 12/08/2009 }\end{array}$ \\
\hline \multicolumn{2}{|c|}{$\begin{array}{l}\text { * Is the NCR operational equipment needed for Conditional } \\
\text { Use? } \\
\text { YYes No }\end{array}$} \\
\hline \multicolumn{2}{|c|}{ Optional Internal Area of Responsibility: } \\
\hline \multicolumn{2}{|c|}{$\begin{array}{l}{ }^{*} \text { Facility Manager: } \\
\text { Petti, David A }\end{array}$} \\
\hline $\begin{array}{l}\text { *Does } 1 \\
\text { ilems? } \\
\text { Yes }\end{array}$ & ivolve suspect/counterfeit \\
\hline
\end{tabular}

*Does this NCR pertain to Waste Containers, Waste Packaging, or Packaging and Transportation aclivities? $\bigcirc$ Yes No

Method of Segregation:

Material is located at ORNL and is segregated from other fuel batches to prevent inadvertant use

Method of Identification:

Clearly lable by batch number

* Lead Disposition Evaluator:

Barnes, Charles $M$

Additional Disposition Evaluator(s):

(These evaluators verify and concur the disposition of NCR.)

Additional Notification:

\section{QE Red Tag Process}

Quality Engineer (QE):

Roberts, Gary D

Tagging information/Other Melhods:

\section{This block is intentionally left blank.}

Other means of Tag Identification:

Organization $\quad$ Phone:

6-8961

Date Processed:

07/30/2009

Disposition

Lead Disposition Evaluator:

Barnes, Charles M

\begin{tabular}{|l|l|}
\hline Organization: & Phone: \\
C700 & $6-0864$ \\
\hline
\end{tabular}

Date Disposition sent for

approval:

$12 / 08 / 2009$

*NCR Disposition:

Use As Is

$\bigcirc$ Reject

Repalr

Multiple Disposition

* Multiple Disposition Documentation:

1. LEU06 and LEU07 compacts: Do not use for AGR-2 fuel because of high uranium contamination. However, because full characterization has been performed on these compacts

Rework and the kernels and coated particles that they contain, LEUO6 
*Does Disposition represent Design Change? Yes No compacts should be retained in storage at INL and ORNL for possible future uses. These uses include measurement of thermal conductivity or other compact properties, tests of PIE methods, and tests to better determine fuel specification limits. 2. LEU09 and LEU11 compacts: Use as is. See justification below.

*Does this item require a Unreviewed Safety Question (USQ) screening and evaluation?

Identify as-built drawings and other documentation:( ${ }^{*}$ For Use-As-Is and Repair)

N/A

Method of Disposal:( ${ }^{*}$ For Reject)

N/A

Technical Justification:( ${ }^{*}$ For Use-As-Is and Repair)

Justification for use as is for LEU09 and LEU11 compacts: Uranium equivalent to 2 defective particles has been found in 60 LEU09 compacts; this level is low enough to permil use of these compacts in the AGR-2 experiment, although the level may not meet the fuel uranium contamination specification limit. The final determination of whether LEU09 compacts meet the uranium contamination specification will be made after analyses are complele of ariother 40 compacts. No uranium contamination has been found in the analysls of 40 LEU11 compacts. 60 additlonal LEU11 compacts are being analyzed. Past analyses of multiple sets of 20 compacts show very little variation in results from one set to the next because of the large number of particles in each set of 20 compacts.

Technical requirements and acceptance criteria to be used for repair work:

N/A

Inspections and Verification Criteria for acceptability of repair or rework:

N/A

Other Documents or QA records requiring the change:

N/A

If this nonconforming item is associated with, or caused by, a program, procedure, or process problem, document the issue in accordance with LWP-13840:

N/A

Disposition Concurrence/Approval

\begin{tabular}{l|l}
\hline Approval RM(Signature) & Concurrence/Approval \\
Croson, Diane V & QE(Signature) \\
Diane V Croson & Roberts, Gary D \\
$12 / 08 / 2009$ & Gary D Roberts \\
& $12 / 08 / 2009$ \\
\hline
\end{tabular}

Implementatlon Completion - RM

\begin{tabular}{l|l|l|l}
\hline $\begin{array}{l}\text { Responsible Manager (RM): } \\
\text { Croson, Diane V }\end{array}$ & $\begin{array}{l}\text { Organization } \\
\text { C700 }\end{array}$ & $\begin{array}{l}\text { Phone: } \\
6-3402\end{array}$ & Date Completion: \\
\hline
\end{tabular}

The Disposition as approved has been completed and implemented: $\cup$ Yes $\cup$ N/A

Implementing Documentation:

Attachments/Comments<smiles>[C]1CC2C[Te]1C2</smiles>

PAAA 44791 (AGR-2 compacts).pdf

Revision History 12/08/2009 04:30 PM : Diane V Croson as an RM concurred the Disposition and signed off. 12/08/2009 04:20 PM : Gary D Roberts as a QE concurred the Disposition and signed off. 12/08/2009 02:37 PM : Charles M Barnes completed NCR Disposition and submitted to Croson, Diane V; Roberts, Gary D for their concurrence and approval.

12/08/2009 07:52 AM : Diane V Croson completed Notification Process and notified Roberts, Gary D; Soto, Rafael; Smith, Angela J; Armour, Kimberly Jo; Smith, Angela J; Petti, David A; Barnes, Charles M

12/08/2009 07:44 AM : Gary D Roberts completed screening and forwarded to Croson, Diane V for Notification process.

12/08/2009 07:34 AM : Diane V Croson completed screening and forwarded to Roberts, Gary D for QE Screening.

12/08/2009 07:26 AM : Angela J Smith changed the RM from Cox, John R to Croson, Diane V

08/04/2009 03:26 PM : Charles M Barnes submitted NCR to RM Cox, John R for screening. 
The following fields are general purpose public use. Any data entered here is not related to NCR process and solely used for one's individual need. Integrity of the data is not guarantied since it can be replaced by any user randomly.

FIELD A:

(Field Name: FIELDA, type Text)

FIELD B:

(Field Name: FIELDb, type Text) 
230.01 Revision date 09/30/2003

Initiator:

Barnes, Charles M

\section{Control of Nonconforming Items Nonconformance Documentation}

Documentation

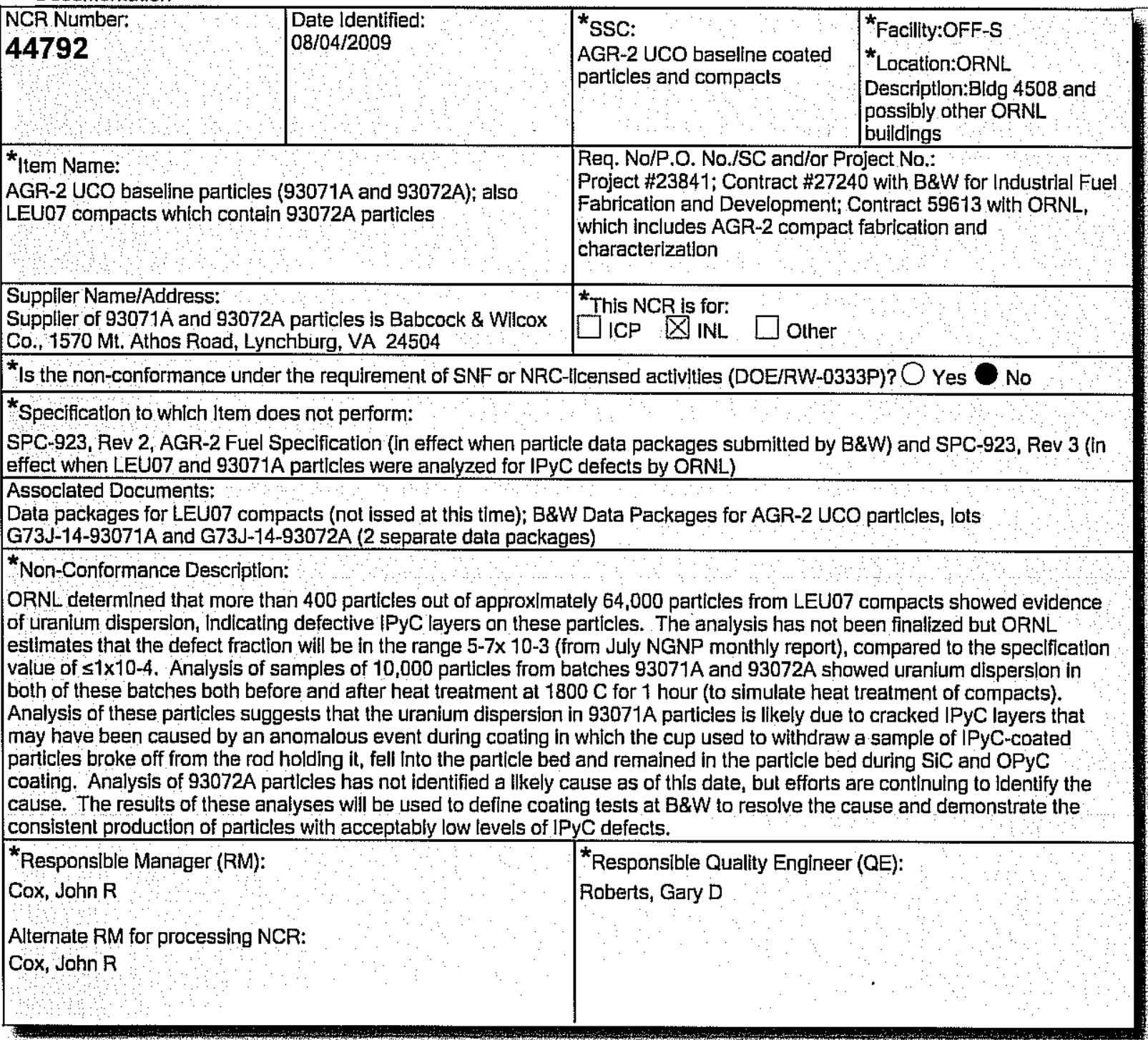

Next Activity: Implementation Completion - RM

Actionee: Cox, John R

Date Due:

Screening - Responsible Manager

Responsible Manager (RM):

Cox, John R

Work Org.:

Work Phone:

059914

C700

6-0864 
*Does the NCR require Stop Work?

Y Yes no

${ }^{\star}$ Does this NCR support Environmental Requirements? $\bigcirc$ Yes $\odot$ No

RM Comments:

None

Screening - Quality EngIneer

\begin{tabular}{l|l|l}
\hline Quality Engineer (QE): & Organizalion & Phone:
\end{tabular}

\begin{tabular}{l|l|l} 
Roberts, Gary D & W560 & $6-8961$ \\
\hline
\end{tabular}

Date Screened:

$08 / 05 / 2009$

* Is the NCR valid? Yes $O$ No

Quality Comments:

None

Notification - RM

Responsible Manager (RM):

Cox, John R C700

ORPS Report Number: N/A

*Area of Responsibility:

INL NGNP

Cognizant D|rector:

Soto, Rafael

Cognizant Director's Alternate(s):

Smith, Angela J; Armour, Kimberly Jo

Compliance Coordinator(s) to determine Price Anderson

(PAAA) noncompliance:

Smith, Argela J

Phone: 6-5585

Date Notified: 08/05/2009

* Is the NCR operational equipment needed for Conditlonal Use?

Y Yes No

Optional Internal Area of Responsibility:

*Does this NCR pertain to Waste Containers, Waste Packaging, or Packaging and Transportation activities? $\bigcirc$ Yes $\bigcirc$ No Method of Segregation:

Material is located at ORNL and is segregated from other fuel batches to prevent inadvertent use

Method of Identification:

clearly lable by batch number

*Lead Disposition Evaluator:

Barnes, Charles M

Additional Disposition Evaluator(s):

(These evaluators verify and concur the dispositlon of NCR.)

Addittonal Notification:

\section{QE Red Tag Process}

Quality Engineer (QE):

Roberts, Gary D

Organizalion

W560

Phone:

$6-8961$

Date Processed:

$08 / 04 / 2009$

*Facility Manager:

Pettl, David A

*Does the non-conformance involve suspect/counterfeit items?

Yes No

Taging information/Other Methods:

Item is not tagged with Red Tag. Segregation and batch number used to identify non-conforming material at ORNL. Other means of Tag Identification:

N/A

DIsposittion

Lead Disposition Evaluator:

Barnes, Charles M

Organization:

C700 $6-0864$

Date Disposition sent for approval:

*NCR Disposition:

Use As is

$08 / 13 / 2009$

Repair

Reject

ORework

Multiple Disposition

* Multiple Disposition Documentation:

1. 93071A partlcles: Do not use for AGR-2 fuel because of anomalous event during coating and high IPyC layer defect fraction. Keep retainer and archive samples of $93071 \mathrm{~A}$ particles at ORNL and B\&W but dispose of remalnder as needed by ORNL to keep enriched uranium inventory within facility limits. Disposal can be through normal ORNL waste dlsposal channels, or by returning to B\&W. B\&W would store this material and evaluate whether to recover the enriched uranium in this material along with other scrap materials from 
the AGR program when sufficient inventory builds up or at program close out.

2. 93072A particles: Very limited quantities of these particles remain and should be archived and stored at ORNL with other archived AGR material. The purpose of retaining these particles is for possible future analyses to better determine the cause of the defects and to use for purposes unrelated to the particle defects.

3. LEU07 compacts: Due to the high fraction of IPyC defects in the particles contained in these compacts, the compacts should be rejected from use in the AGR-2 experiment. However, due to the full characterization that has been performed on these compacts and the kernels and particles they contain, LEU07 compacts should be retained in storage at INL and ORNL for possible future uses. These posslble uses include measurements of thermal conductivity or other compact properties, tests of PIE methods, and irradiation experimetns to determine the consequences of high uranium dispersion and establish a better basis for the defective IPyC fuel specification.

*Does Dispositlon represent Design Change?

*Does this item require a Unreviewed Safety Question (USQ)

screening and evaluation?

Yes No

Identify as-built drawings and other documentation:( ${ }^{\star}$ For Use-As-Is and Repalr)

N/A

Method of Disposal:( ${ }^{*}$ For Reject)

Disposal will be in accordance with waste disposal processes at ORNL and/or B\&W.

Technical Justification:( ${ }^{\star}$ For Use-As-Is and Repair)

N/A

Technical requirements and acceptance criteria to be used for repalr work:

N/A

Inspections and Verification Criteria for acceptability of repair or rework:

N/A

Other Documents or QA records requiring the change:

N/A

If this nonconforming item is associated with, or caused by, a program, procedure, or process problem, document the issue in accordance with LWP-13840:

N/A

Disposttion Concurrence/Approval

Approval RM(Signature) $\quad$ Concurrence/Approval

Cox, John $R$

John $R$ Cox

$08 / 13 / 2009$
QE(Signature)

Roberts, Gary D

Gary D Roberts

$08 / 13 / 2009$
This block is intentionally left blank.
This block is intentionally left blank.

Implementation Completion - RM

\begin{tabular}{l|l|l|l}
\hline $\begin{array}{l}\text { Responsible Manager (RM): } \\
\text { Cox, John R }\end{array}$ & $\begin{array}{l}\text { Organization } \\
\text { C700 }\end{array}$ & $\begin{array}{l}\text { Dhone: } \\
6-5585\end{array}$ & \\
\hline
\end{tabular}

The Disposition as approved has been completed and implemented: $\bigcirc$ Yes $O$ N/A

Implementing Documentation:

Attachments/Comments<smiles>[C+]1[CH][CH]1</smiles>

PAAA 44792 (AGR-2 particles).pdf

Revision History 08/13/2009 04:14 PM : Gary D Roberts as a QE concurred the Disposition and signed off. 08/13/2009 02:12 PM : John R Cox as an RM concurred the Disposition and signed off. 
08/13/2009 01:57 PM : Charles M Bames completed NCR Disposition and submitted to Cox, John R; Roberts, Gary D for their concurrence and approval.

08/05/2009 12:23 PM : John R Cox completed Notification Process and notified Roberts, Gary D; Soto, Rafael; Smith, Angela J; Smith, Angela J; Petti, David A; Barnes, Charles M

08/05/2009 12:15 PM : Gary D Roberts completed screening and forwarded to Cox, John R for Notification process. 08/05/2009 12:03 PM : John R Cox compleled screening and forwarded to Roberts, Gary D for QE Screening. 08/04/2009 04:15 PM : Charles M Bames submitted NCR to RM Cox, John R for screening.

The following fields are general purpose public use. Any data entered here is not related to NCR process and solely used for one's individual need. Integrity of the data is not guarantied since it can be replaced by any user randomly.

FIELD A:

(Field Name: FIELDA, type Text)

FIELD B:

(Fleld Name: FIELDb, type Text) 